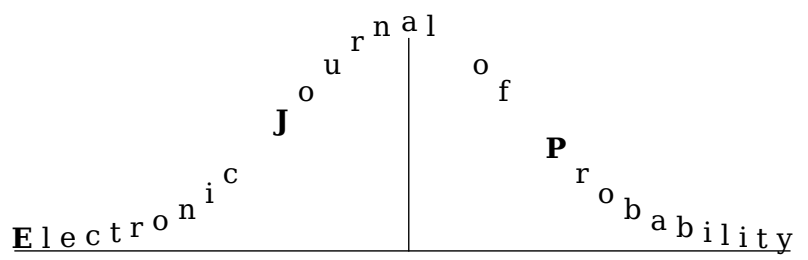

Electron. J. Probab. 25 (2020), article no. 106, 1-66.

ISSN: 1083-6489 https://doi.org/10.1214/20-EJP507

\title{
On the boundary local time measure of super-Brownian motion
}

\author{
Jieliang Hong*
}

\begin{abstract}
In [9] the Hausdorff dimension, $d_{f}$, of $\partial \mathcal{R}$, the topological boundary of the range of super-Brownian motion for dimensions $d=2,3$ was found; $d_{f}=4-2 \sqrt{2}$ if $d=2$, and $d_{f}=(9-\sqrt{17}) / 2$ if $d=3$. We will refine these dimension estimates in a number of ways.

If $L^{x}$ is the total occupation local time of $d$-dimensional super-Brownian motion, $X$, for $d=2$ and $d=3$, we construct a random measure $\mathcal{L}$, called the boundary local time measure, as a rescaling of $L^{x} e^{-\lambda L^{x}} d x$ as $\lambda \rightarrow \infty$, thus confirming a conjecture of [19] and further show that the support of $\mathcal{L}$ equals $\partial \mathcal{R}$. This latter result uses a second construction of a boundary local time $\widetilde{\mathcal{L}}$ given in terms of exit measures and we prove that $\widetilde{\mathcal{L}}=c \mathcal{L}$ a.s. for some constant $c>0$. We derive reasonably explicit first and second moment measures for $\mathcal{L}$ in terms of negative dimensional Bessel processes and use them with the energy method to give a more direct proof of the lower bound of the Hausdorff dimension of $\partial \mathcal{R}$ in [9]. The construction requires a refinement of the $L^{2}$ upper bounds in [19] and [9] to exact $L^{2}$ asymptotics. The methods also refine the left tail bounds for $L^{x}$ in [19] to exact asymptotics. We conjecture that the $d_{f}$-dimensional Minkowski content of $\partial \mathcal{R}$ is equal to the total mass of the boundary local time $\mathcal{L}$ up to some constant.
\end{abstract}

Keywords: super-Brownian motion; local time; exit measure; boundary local time measure. MSC2020 subject classifications: 60G57; 60J68; 60H30; 35J75; 60J80.

Submitted to EJP on March 3, 2020, final version accepted on August 10, 2020.

Supersedes arXiv: 2001.09137.

\section{Introduction and main results}

\subsection{Introduction}

Let $M_{F}=M_{F}\left(\mathbb{R}^{d}\right)$ be the space of finite measures on $\left(\mathbb{R}^{d}, \mathfrak{B}\left(\mathbb{R}^{d}\right)\right)$ equipped with the topology of weak convergence of measures. A super-Brownian motion (SBM) $\left(X_{t}, t \geq 0\right)$ starting at $X_{0} \in M_{F}$ is a continuous $M_{F}$-valued strong Markov process defined on some filtered probability space $\left(\Omega, \mathcal{F}, \mathcal{F}_{t}, P\right)$ described below and we let $\mathbb{P}_{X_{0}}$ denotes any

\footnotetext{
${ }^{*}$ Department of Mathematics, University of British Columbia, Canada. E-mail: j lhong@math.ubc.ca
} 
probability under which $X$ is as above. We write $\mu(\phi)=\int \phi(x) \mu(d x)$ for any measure $\mu$ and take our branching rate to be one so that for any non-negative bounded Borel functions $\phi, f$ on $\mathbb{R}^{d}$,

$$
\mathbb{E}_{X_{0}}\left(\exp \left(-X_{t}(\phi)-\int_{0}^{t} X_{s}(f) d s\right)\right)=\exp \left(-X_{0}\left(V_{t}(\phi, f)\right)\right) .
$$

Here $V_{t}(x)=V_{t}(\phi, f)(x)$ is the unique solution of the mild form of

$$
\frac{\partial V}{\partial t}=\frac{\Delta V_{t}}{2}-\frac{V_{t}^{2}}{2}+f, \quad V_{0}=\phi
$$

that is,

$$
V_{t}=P_{t}(\phi)+\int_{0}^{t} P_{s}\left(f-\frac{V_{t-s}^{2}}{2}\right) d s .
$$

In the above $\left(P_{t}\right)$ is the semigroup of standard $d$-dimensional Brownian motion. See Chapter II of [20] for the above and further properties.

It is known that the extinction time of $X$ is a.s. finite (see, e.g., Chp II.5 in [20]). The total occupation time measure of $X$ is the (a.s. finite) measure defined as

$$
I(A)=\int_{0}^{\infty} X_{s}(A) d s .
$$

Let $S(\mu)=\operatorname{Supp}(\mu)$ denote the closed support of a measure $\mu$. We define the range, $\mathcal{R}$, of $X$ to be $\mathcal{R}=\operatorname{Supp}(I)$. In dimensions $d \leq 3$, the occupation measure $I$ has a density, $L^{x}$, which is called (total) local time of $X$, that is,

$$
I(f)=\int_{0}^{\infty} X_{s}(f) d s=\int_{\mathbb{R}^{d}} f(x) L^{x} d x \text { for all non-negative measurable } f .
$$

Moreover, $x \mapsto L^{x}$ is lower semicontinuous, is continuous on $S\left(X_{0}\right)^{c}$, and for $d=1$ is globally continuous (see Theorems 2 and 3 of [23]). Thus one can see that in dimensions $d \leq 3$,

$$
\mathcal{R}=\overline{\left\{x: L^{x}>0\right\}},
$$

and $\mathcal{R}$ is a closed set of positive Lebesgue measure. In dimensions $d \geq 4, \mathcal{R}$ is a Lebesgue null set of Hausdorff dimension 4 for SBM starting from $\delta_{0}$ (see Theorem 1.4 of [2]), which explains our restriction to $d \leq 3$ in this work.

We will largely be considering the case when $X_{0}=\delta_{0}$. The Hausdorff dimensions of the boundaries of SBM have been studied in [19] and [9]. Let $\partial \mathcal{R}$ be the topological boundary of the range $\mathcal{R}$ and define $F$ to be the boundary of the set where the local time is positive, i.e. $F:=\partial\left\{x: L^{x}>0\right\}$. Let dim denote the Hausdorff dimension and introduce:

$$
p=p(d)=\left\{\begin{array}{ll}
3 & \text { if } d=1 \\
2 \sqrt{2} & \text { if } d=2 \\
\frac{1+\sqrt{17}}{2} & \text { if } d=3,
\end{array} \text { and } \alpha=\frac{p-2}{4-d}= \begin{cases}1 / 3 & \text { if } d=1 \\
\sqrt{2}-1 & \text { if } d=2 \\
\frac{\sqrt{17}-3}{2} & \text { if } d=3 .\end{cases}\right.
$$

Theorem 1.1 ([19], [9]). With $\mathbb{P}_{\delta_{0}}$-probability one,

$$
\operatorname{dim}(F)=\operatorname{dim}(\partial \mathcal{R})=d_{f}:=d+2-p= \begin{cases}0 & \text { if } d=1 \\ 4-2 \sqrt{2} \approx 1.17 & \text { if } d=2 \\ \frac{9-\sqrt{17}}{2} \approx 2.44 & \text { if } d=3\end{cases}
$$


It is also natural to consider SBM under the canonical measure $\mathbb{N}_{x_{0}}$. Recall from Section II.7 in [20] that $\mathbb{N}_{x_{0}}$ is a $\sigma$-finite measure on $C\left([0, \infty), M_{F}\right)$, which is the space of continuous $M_{F}\left(\mathbb{R}^{d}\right)$-valued paths furnished with the compact-open topology, such that if we let $\Xi=\sum_{i \in I} \delta_{\nu^{i}}$ be a Poisson point process on $C\left([0, \infty), M_{F}\right)$ with intensity $\mathbb{N}_{X_{0}}(d \nu)=\int \mathbb{N}_{x}(d \nu) X_{0}(d x)$, then

$$
X_{t}=\sum_{i \in I} \nu_{t}^{i}=\int \nu_{t} \Xi(d \nu), t>0,
$$

has the law, $\mathbb{P}_{X_{0}}$, of a super-Brownian motion $X$ starting from $X_{0}$. In this way, $\mathbb{N}_{x_{0}}$ describes the contribution of a cluster from a single ancestor at $x_{0}$ and the superBrownian motion is then obtained by a Poisson superposition of such clusters. We refer the readers to Theorem II.7.3(c) in [20] for more details. The existence of the local time $L^{x}$ under $\mathbb{N}_{x_{0}}$ will follow from this decomposition and the existence under $\mathbb{P}_{\delta_{x_{0}}}$. Therefore the local time $L^{x}$ under $\mathbb{P}_{X_{0}}$ may be decomposed as

$$
L^{x}=\sum_{i \in I} L^{x}\left(\nu^{i}\right)=\int L^{x}(\nu) \Xi(d \nu)
$$

The global continuity of local times $L^{x}$ under $\mathbb{N}_{x_{0}}$ is given in Theorem 1.2 of [6]. It is not surprising that Theorem 1.1 continues to hold under the canonical measure.

Theorem 1.2 ([19], [9]). $\mathbb{N}_{0}$-a.e. $\operatorname{dim}(F)=\operatorname{dim}(\partial \mathcal{R})=d_{f}$.

The definition of $F$ is natural from an analytical perspective but the topological boundary $\partial \mathcal{R}$ is a more natural random set from a geometrical point of view. One can check that

$$
\partial \mathcal{R} \subset F .
$$

In $d=1$, it has been shown in Theorem 1.7 in [19] and Theorem 1.4 in [7] that there exist random variables $L$ and $R$ such that

$$
F=\partial \mathcal{R}=\{\mathrm{L}, \mathrm{R}\} \text { where } \mathrm{L}<0<\mathrm{R}, \quad \mathbb{N}_{0} \text {-a.e. and } \mathbb{P}_{\delta_{0}} \text {-a.s. }
$$

Whether or not $F=\partial \mathcal{R}$ remains open in $d=2$ and $d=3$. Given the simple nature of $F$ and $\partial \mathcal{R}$ in $d=1$, we largely will focus on $d=2$ and $d=3$ in what follows.

Our main goal in this paper is to construct a random measure on $\partial \mathcal{R}$ or $F$. Recall $\alpha$ from (1.3). For any $\lambda>0$, under $\mathbb{P}_{\delta_{0}}$ and $\mathbb{N}_{0}$ we define a random measure $\mathcal{L}^{\lambda}$ on $\mathbb{R}^{d}$ by

$$
d \mathcal{L}^{\lambda}(x)=\lambda^{1+\alpha} L^{x} e^{-\lambda L^{x}} d x .
$$

The two authors in [19] conjecture that as $\lambda \rightarrow \infty, \mathcal{L}^{\lambda}$ converges in probability in the space $M_{F}\left(\mathbb{R}^{d}\right)$ to a finite measure $\mathcal{L}$ which necessarily is supported on $F$. In this paper, we confirm this conjecture and further show that the support of $\mathcal{L}$ is precisely $\partial \mathcal{R}$.

Convention on functions and constants Constants whose value is unimportant and may change from line to line are denoted $C, c, c_{d}, c_{1}, c_{2}, \ldots$, while constants whose values will be referred to later and appear initially in say, Lemma i.j are denoted $c_{i . j}$, or $\underline{c}_{i . j}$ or $C_{i . j}$.

Notation Let $M_{F}$ be equipped with any complete metric $d_{0}$ inducing the weak topology and let $\left\{\mu_{t}, t \in T\right\}$ be a collection of $M_{F}$-valued random vectors. We use $\mu_{t} \stackrel{P}{\rightarrow} \mu_{t_{0}}$ as $t \rightarrow t_{0}$ to denote the convergence in probability under $\mathbb{P}_{X_{0}}$ if for any $\varepsilon>0$, we have $\mathbb{P}_{X_{0}}\left(d_{0}\left(\mu_{t}, \mu_{t_{0}}\right)>\varepsilon\right) \rightarrow 0$ as $t \rightarrow t_{0}$. We slightly abuse the notation and use $\mu_{t} \stackrel{P}{\rightarrow} \mu_{t_{0}}$ as $t \rightarrow t_{0}$ to denote the convergence in measure under $\mathbb{N}_{X_{0}}$ if for any $\varepsilon>0$, we have $\mathbb{N}_{X_{0}}\left(\left\{d_{0}\left(\mu_{t}, \mu_{t_{0}}\right)>\varepsilon\right\} \cap A\right) \rightarrow 0$ as $t \rightarrow t_{0}$ where $A$ is any measurable set with $\mathbb{N}_{X_{0}}(A)<\infty$. 


\subsection{Main results}

Theorem 1.3. Let $d=2$ or 3 . Under both $\mathbb{N}_{0}$ and $\mathbb{P}_{\delta_{0}}$, there exists a random measure $\mathcal{L} \in M_{F}\left(\mathbb{R}^{d}\right)$, supported on $\partial \mathcal{R}$, such that $\mathcal{L}^{\lambda} \stackrel{P}{\rightarrow} \mathcal{L}$ as $\lambda \rightarrow \infty$ and there is a sequence $\lambda_{n} \rightarrow \infty$ such that $\mathcal{L}^{\lambda_{n}} \rightarrow \mathcal{L}$ a.s. as $n \rightarrow \infty$.

In Corollary 1.7 below we show that the support of $\mathcal{L}$ is exactly $\partial \mathcal{R}$ and so $\mathcal{L} \neq 0$ $\mathbb{N}_{0}$-a.e. and $\mathbb{P}_{\delta_{0}}$-a.s. A different problem is studied in [11] where a random measure is constructed on the boundary of the zero set of the density $X(t, x)$ for any fixed $t>0$ in $d=1$.

Next we consider the case under $\mathbb{N}_{X_{0}}$ and $\mathbb{P}_{X_{0}}$ for general initial condition $X_{0}$. Since the above theorem holds under $\mathbb{N}_{x}$ for any $x$ by translation invariance of SBM, and $\mathbb{N}_{X_{0}}(\cdot)=\int \mathbb{N}_{x}(\cdot) X_{0}(d x)$, it is easy to see that the above result continues to hold under $\mathbb{N}_{X_{0}}$ for any $X_{0}$. However, the case under $\mathbb{P}_{X_{0}}$ is somehow more delicate-instantaneous extinction at time $t=0$ will make the behavior of $\partial \mathcal{R} \cap S\left(X_{0}\right)$ quite different from that under $\mathbb{P}_{\delta_{0}}$ and $\mathbb{N}_{0}$; see Proposition 1.6 and Remark 1.8(b) of [19] for such examples. Therefore under $\mathbb{P}_{X_{0}}$ we will restrict our interest to $S\left(X_{0}\right)^{c}$. For any $\lambda>0$, under $\mathbb{P}_{X_{0}}$ we define a random measure $\mathcal{L}^{\lambda}$ supported on $S\left(X_{0}\right)^{c}$ by

$$
d \mathcal{L}^{\lambda}(x)=\lambda^{1+\alpha} L^{x} e^{-\lambda L^{x}} 1\left(x \in S\left(X_{0}\right)^{c}\right) d x .
$$

Notation For any $\delta>0$ and any set $K$, we let $K^{\geq \delta}=\{x: d(x, K) \geq \delta\}$ where $d(x, K)=\inf \{|x-y|: y \in K\}$. Similarly we define $K^{>\delta}, K^{\leq \delta}$ and $K^{<\delta}$. For any measure $\mu$ and any set $K$, we use $\left.\mu\right|_{K}(\cdot) \equiv \mu(\cdot \cap K)$ to denote the restriction of $\mu$ to $K$.

Theorem 1.4. Let $d=2$ or 3 and let $X_{0} \in M_{F}\left(\mathbb{R}^{d}\right)$. Under $\mathbb{P}_{X_{0}}$ there exists a $\sigma$-finite random measure $\mathcal{L}$, supported on $\partial \mathcal{R} \cap S\left(X_{0}\right)^{c}$, such that for any $k \geq 1$, we have $\left.\left.\mathcal{L}^{\lambda}\right|_{S\left(X_{0}\right) \geq 1 / k} \stackrel{P}{\rightarrow} \mathcal{L}\right|_{S\left(X_{0}\right) \geq 1 / k}$ as $\lambda \rightarrow \infty$ and there is a sequence $\lambda_{n} \rightarrow \infty$ such that $\left.\left.\mathcal{L}^{\lambda_{n}}\right|_{S\left(X_{0}\right) \geq 1 / k} \rightarrow \mathcal{L}\right|_{S\left(X_{0}\right) \geq 1 / k}, \forall k \geq 1$ a.s.

Remark 1.5. (a) The behavior of $\partial \mathcal{R}$ on the boundary of $S\left(X_{0}\right)$ depend largely on the mass distribution of $X_{0}$ and is still quite different from that under $\mathbb{N}_{0}$ and $\mathbb{P}_{\delta_{0}}$. In the proof we first give the existence of a finite measure $l_{k}$ by restricting our interest to $S\left(X_{0}\right)^{\geq 1 / k}$ for any $k \geq 1$ and then construct a $\sigma$-finite measure $\mathcal{L}$ supported on $S\left(X_{0}\right)^{c}$ by defining $\left.\mathcal{L}\right|_{S\left(X_{0}\right) \geq 1 / k}=l_{k}$ for any $k \geq 1$. In most cases we will only be considering the properties of $\mathcal{L}$ on sets with positive distance away from $S\left(X_{0}\right)$ and the above theorem suffices for our purposes.

(b) One sufficient condition on $X_{0}$ to give the a.s finiteness of $\mathcal{L}(1)$ goes back to the renormalization of local times in $d=2$ or 3 (see [6]). For example in $d=2$, if we have $\inf _{x \in S\left(X_{0}\right)} \int \log ^{+}(1 /|y-x|) X_{0}(d y)=\infty$, then Theorem 1.11 of [6] will imply that $\mathbb{P}_{X_{0}}$-a.s. there is some $\delta>0$ so that $S\left(X_{0}\right)^{<\delta} \subset \operatorname{Int}(\mathcal{R})$, and therefore $S\left(X_{0}\right)^{<\delta}$ is not in the support of $\mathcal{L}$. Hence $\mathcal{L}=\left.\mathcal{L}\right|_{S\left(X_{0}\right) \geq \delta}$ and the a.s. finiteness of $\mathcal{L}(1)$ follows.

Theorem 1.6. $\mathbb{P}_{X_{0}}$-a.s. and $\mathbb{N}_{X_{0}}$-a.e. for any open set $U \subset S\left(X_{0}\right)^{c}$,

$$
U \cap \partial \mathcal{R} \neq \emptyset \Rightarrow \mathcal{L}(U)>0
$$

In particular we have $\mathbb{P}_{X_{0}}$-a.s. that $\operatorname{Supp}(\mathcal{L})=S\left(X_{0}\right)^{c} \cap \partial \mathcal{R}$ and

$$
S\left(X_{0}\right)^{c} \cap \partial \mathcal{R} \neq \emptyset \Rightarrow \mathcal{L}>0 .
$$

Note under $\mathbb{P}_{X_{0}}$ we will be working on the space $S\left(X_{0}\right)^{c}$ and so the set $S\left(X_{0}\right)^{c} \cap \partial \mathcal{R}$ will be a closed set in $S\left(X_{0}\right)^{c}$. The hypothesis in (1.11) is necessary-an example is given in Proposition 1.5 of [19] where it fails with positive probability.

Let $B\left(x_{0}, \varepsilon\right)=B_{\varepsilon}\left(x_{0}\right)=\left\{x:\left|x-x_{0}\right|<\varepsilon\right\}$ and set $B_{\varepsilon}=B(\varepsilon)=B_{\varepsilon}(0)$. 
Corollary 1.7. $\mathbb{P}_{\delta_{0}}$-a.s. and $\mathbb{N}_{0}$-a.e. for any open set $U$,

$$
U \cap \partial \mathcal{R} \neq \emptyset \Rightarrow \mathcal{L}(U)>0 .
$$

In particular, $\operatorname{Supp}(\mathcal{L})=\partial \mathcal{R}$ and $\mathcal{L}>0, \mathbb{P}_{\delta_{0}}$-a.s. and $\mathbb{N}_{0}$-a.e.

Proof. We know from the proof of Corollary 1.4 and Theorem 1.5 of [9] that $\mathbb{P}_{\delta_{0}}$-a.s. and $\mathbb{N}_{0}$-a.e. there exists some $\delta>0$ such that $L^{x}>0$ for all $|x|<\delta$ and so $0 \notin \partial \mathcal{R}$, which implies

$$
U \cap \partial \mathcal{R} \neq \emptyset \Rightarrow(U \backslash\{0\}) \cap \partial \mathcal{R} \neq \emptyset .
$$

Then we may apply Theorem 1.6 with $U \backslash\{0\}$ in place of $U$ to complete the proof of (1.12). Next for any $x \in \partial \mathcal{R}$, take $U=B(x, \varepsilon)$ for any $\varepsilon>0$ and use the above to get $\partial \mathcal{R} \subset \operatorname{Supp}(\mathcal{L})$. Together with Theorem 1.3 we conclude $\operatorname{Supp}(\mathcal{L})=\partial \mathcal{R}, \mathbb{P}_{\delta_{0}}$-a.s. and $\mathbb{N}_{0}$-a.e. By (1.12), it follows immediately that $\mathcal{L}>0, \mathbb{P}_{\delta_{0}}$-a.s. and $\mathbb{N}_{0}$-a.e.

Now we proceed to the first and second moment measures of $\mathcal{L}$. Define

$$
\mu=\left\{\begin{array}{ll}
-1 / 2 & \text { if } d=1 \\
0 & \text { if } d=2 \\
1 / 2 & \text { if } d=3,
\end{array} \text { and } \nu=\sqrt{\mu^{2}+4(4-d)}= \begin{cases}7 / 2 & \text { if } d=1 \\
2 \sqrt{2} & \text { if } d=2 \\
\sqrt{17} / 2 & \text { if } d=3\end{cases}\right.
$$

so that (recall (1.3)) $d=2+2 \mu$ and $p=\mu+\nu$. Let $\hat{P}_{x}^{(2-2 \nu)}$ denote the law of the $d$-dimensional process $\left\{Y_{t}: t \geq 0\right\}$ such that

$$
\left\{\begin{array}{l}
Y_{t}=x+\hat{B}_{t}+\int_{0}^{t}(-\nu-\mu) \frac{Y_{s}}{\left|Y_{s}\right|^{2}} d s, \quad t<\tau_{0}, \\
Y_{t}=0, \quad t \geq \tau_{0}
\end{array}\right.
$$

Here $\tau_{0}=\inf \left\{t \geq 0:\left|Y_{t}\right|=0\right\}$ and $\hat{B}$ is a standard $d$-dimensional Brownian motion starting from $x$ under $\hat{P}_{x}^{(2-2 \nu)}$. Remark 1.9(b) below shows why $\hat{P}_{x}^{(2-2 \nu)}$ is well-defined. Let $V^{\infty}(x):=\mathbb{N}_{0}\left(L^{x}>0\right)$ for all $x \neq 0$. For any $x_{1} \neq x_{2}$, we let $\vec{x}=\left(x_{1}, x_{2}\right)$ and define for all $x \neq x_{1}, x_{2}$,

$$
V^{\vec{\infty}, \vec{x}}(x):=\mathbb{N}_{x}\left(\left\{L^{x_{1}}>0\right\} \cup\left\{L^{x_{2}}>0\right\}\right)
$$

For $i=1,2$ we define

$$
U_{i}^{\vec{\infty}, \vec{x}}(x):=\frac{1}{\left|x-x_{i}\right|^{p}} \hat{E}_{x-x_{i}}^{(2-2 \nu)}\left(\exp \left(-\int_{0}^{\tau_{0}}\left(V^{\vec{\infty}, \vec{x}}\left(Y_{s}+x_{i}\right)-V^{\infty}\left(Y_{s}\right)\right) d s\right)\right),
$$

and set

$$
U_{1,2}^{\vec{\infty}, \vec{x}}(x):=-E_{x}\left(\int_{0}^{\infty} \prod_{i=1}^{2} U_{i}^{\vec{\infty}, \vec{x}}\left(B_{t}\right) \exp \left(-\int_{0}^{t} V^{\vec{\infty}, \vec{x}}\left(B_{s}\right) d s\right) d t\right),
$$

where $B$ is a $d$-dimensional Brownian motion starting from $x$ under $P_{x}$.

Theorem 1.8. (a) There is some constant $K_{1.8}>0$ such that for any nonnegative measurable $\phi: \mathbb{R}^{d} \rightarrow \mathbb{R}$, we have

$$
\mathbb{N}_{0}\left(\int \phi(x) d \mathcal{L}(x)\right)=K_{1.8} \int|x|^{-p} \phi(x) d x .
$$

(b) For any nonnegative measurable $h: \mathbb{R}^{d} \times \mathbb{R}^{d} \rightarrow \mathbb{R}$, we have

$$
\mathbb{N}_{0}((\mathcal{L} \times \mathcal{L})(h))=K_{1.8}^{2} \int h\left(x_{1}, x_{2}\right)\left(-U_{1,2}^{\vec{\infty}, \vec{x}}(0)\right) d x_{1} d x_{2}
$$


Moreover, there is some constant $c_{1.8}>0$ such that

$$
\begin{aligned}
& \mathbb{N}_{0}\left(\int h\left(x_{1}, x_{2}\right) d \mathcal{L}\left(x_{1}\right) d \mathcal{L}\left(x_{2}\right)\right) \\
& \quad \leq K_{1.8}^{2} \int c_{1.8}\left(\left|x_{1}\right|^{-p}+\left|x_{2}\right|^{-p}\right)\left|x_{1}-x_{2}\right|^{2-p} h\left(x_{1}, x_{2}\right) d x_{1} d x_{2} .
\end{aligned}
$$

Remark 1.9. (a) The superscript $2-2 \nu<0$ on $\hat{P}_{x}^{(2-2 \nu)}$ is used to indicate the fact that $\left\{\left|Y_{s}\right|, s \geq 0\right\}$ under $\hat{P}_{x}^{(2-2 \nu)}$ is a stopped Bessel process of dimension $2-2 \nu$ starting from $|x|>0$ (see, e.g., (7.9)), thus giving the connection between the moment measures of $\mathcal{L}$ and Bessel process of negative dimension. We refer the reader to [5] for more information on Bessel process of negative dimensions. See also [17] where a connection is made in $d=1$ between the left-most point in the range of SBM and the Bessel process of dimension $2-2 \nu=-5$ where $\nu=7 / 2$ as in (1.13) for $d=1$.

(b) Under $\hat{P}_{x}^{(2-2 \nu)}$, we have $\tau_{0}$ is the hitting time of 0 of a $(2-2 \nu)$-dimensional Bessel process and so with $\hat{P}_{x}^{(2-2 \nu)}$-probability one, $\tau_{0}<\infty$ (see, e.g., Exercise (1.33) in Chp. XI of [21]). For any $\varepsilon>0$, we have the drift in (1.14) is bounded for all $0 \leq t \leq \tau_{\varepsilon}$ and hence the uniqueness of solutions to (1.14) holds for all $0 \leq t \leq \tau_{\varepsilon}$ (see also (7.13)). It then follows by continuity that the uniqueness of solutions to (1.14) will hold for all $0 \leq t \leq \tau_{0}$.

Theorem 1.10. (a) For any nonnegative measurable $\phi: \mathbb{R}^{d} \rightarrow \mathbb{R}$, we have

$$
\mathbb{E}_{X_{0}}(\mathcal{L}(\phi))=K_{1.8} \int_{S\left(X_{0}\right)^{c}} \phi(x) e^{-X_{0}\left(V^{\infty}(x-\cdot)\right)} X_{0}\left(|x-\cdot|^{-p}\right) d x .
$$

(b) For any nonnegative measurable $h: \mathbb{R}^{d} \times \mathbb{R}^{d} \rightarrow \mathbb{R}$, we have

$$
\begin{aligned}
\mathbb{E}_{X_{0}}((\mathcal{L} \times \mathcal{L})(h))= & K_{1.8}^{2} \int_{\left(S\left(X_{0}\right)^{c}\right)^{2}} h\left(x_{1}, x_{2}\right) \\
& e^{-X_{0}\left(V^{\vec{\infty}, \vec{x}}\right)}\left(X_{0}\left(U_{1}^{\vec{\infty}, \vec{x}}\right) X_{0}\left(U_{2}^{\vec{\infty}, \vec{x}}\right)-X_{0}\left(U_{1,2}^{\vec{\infty}, \vec{x}}\right)\right) d x_{1} d x_{2} .
\end{aligned}
$$

Moreover,

$$
\begin{gathered}
\mathbb{E}_{X_{0}}((\mathcal{L} \times \mathcal{L})(h)) \leq K_{1.8}^{2} \int_{\left(S\left(X_{0}\right)^{c}\right)^{2}} h\left(x_{1}, x_{2}\right)\left(X_{0}\left(\left|x_{1}-\cdot\right|^{-p}\right) X_{0}\left(\left|x_{2}-\cdot\right|^{-p}\right)\right. \\
\left.\quad+c_{1.8}\left(X_{0}\left(\left|x_{1}-\cdot\right|^{-p}\right)+X_{0}\left(\left|x_{2}-\cdot\right|^{-p}\right)\right)\left|x_{1}-x_{2}\right|^{2-p}\right) d x_{1} d x_{2} .
\end{gathered}
$$

Now that we have $\operatorname{Supp}(\mathcal{L})=\partial \mathcal{R}$ a.s. under $\mathbb{N}_{0}$ and $\mathbb{P}_{\delta_{0}}$, one immediate application with the above moment measures would be to use the energy method (see, e.g., Theorem 4.27 of [18]) to find the lower bound of the Hausdorff dimension of $\partial \mathcal{R}$.

Theorem 1.11. For any $\eta>0$, we have for all $k \geq 1$,

$$
\begin{aligned}
& \text { (i) } \mathbb{N}_{0}\left(\int 1_{\left\{k^{-1} \leq\left|x_{1}\right|,\left|x_{2}\right| \leq k\right\}}\left|x_{1}-x_{2}\right|^{-(d+2-p-\eta)} \mathcal{L}\left(d x_{1}\right) \mathcal{L}\left(d x_{2}\right)\right)<\infty, \\
& \text { (ii) } \mathbb{P}_{\delta_{0}}\left(\int 1_{\left\{k^{-1} \leq\left|x_{1}\right|,\left|x_{2}\right| \leq k\right\}}\left|x_{1}-x_{2}\right|^{-(d+2-p-\eta)} \mathcal{L}\left(d x_{1}\right) \mathcal{L}\left(d x_{2}\right)\right)<\infty .
\end{aligned}
$$

In particular, $\operatorname{dim}(\partial \mathcal{R}) \geq d+2-p, \mathbb{N}_{0}$-a.e. and $\mathbb{P}_{\delta_{0}}$-a.s.

Proof. For any $k \geq 1$ and $\eta>0$ small, we apply Theorems $1.8(\mathrm{~b})$ and 1.10 (b) with

$$
h\left(x_{1}, x_{2}\right)=\left|x_{1}-x_{2}\right|^{-(d+2-p-\eta)} 1\left(k^{-1} \leq\left|x_{1}\right| \leq k\right) 1\left(k^{-1} \leq\left|x_{2}\right| \leq k\right)
$$


to get (i) and (ii). Take a countable union of null sets to get $\mathbb{N}_{0^{-}}$-a.e. and $\mathbb{P}_{\delta_{0}}$-a.s. that

$$
\int 1_{\left\{k^{-1} \leq\left|x_{1}\right|,\left|x_{2}\right| \leq k\right\}}\left|x_{1}-x_{2}\right|^{-(d+2-p-\eta)} \mathcal{L}\left(d x_{1}\right) \mathcal{L}\left(d x_{2}\right)<\infty, \forall k \geq 1 .
$$

By the compactness of the range of SBM (see, e.g., Corollary III.1.7 of [20] and Theorem IV.7(iii) of [16]) and that $L^{x}$ is strictly positive for $x$ near 0 (see the proof of Corollary 1.7), we can conclude $\mathbb{N}_{0}$-a.e. and $\mathbb{P}_{\delta_{0}}$-a.s. that $\operatorname{Supp}(\mathcal{L})=\partial \mathcal{R} \subset\left\{x: k^{-1} \leq|x| \leq k\right\}$ for $k$ large enough. Therefore it follows from Theorem 4.27 of [18] that $\mathbb{N}_{0}$-a.e. and $\mathbb{P}_{\delta_{0}}$-a.s. $\operatorname{dim}(\partial \mathcal{R}) \geq d+2-p-\eta$. Let $\eta \downarrow 0$ to get the desired result.

Now we say a few words on the ideas underlying Theorem 1.3. For any point $x$ near $F$ and $\partial \mathcal{R}$, its local time $L^{x}$ will either be zero or small and positive, and hence the asymptotics of $\mathbb{P}_{\delta_{0}}\left(0<L^{x}<\varepsilon\right)$ as $\varepsilon \downarrow 0$ will be useful in studying $F$ and $\partial \mathcal{R}$. The Laplace transform of $L^{x}$ derived in Lemma 2.2 of [19] is given by

$$
\mathbb{E}_{X_{0}}\left(e^{-\lambda L^{x}}\right)=\exp \left(-\int \mathbb{N}_{y}\left(1-e^{-\lambda L^{x}}\right) X_{0}(d y)\right)=\exp \left(-\int V^{\lambda}(x-y) X_{0}(d y)\right),
$$

where $V^{\lambda}$ is the unique solution (see Section 2 of [19] and the references given there) to

$$
\frac{\Delta V^{\lambda}}{2}=\frac{\left(V^{\lambda}\right)^{2}}{2}-\lambda \delta_{0}, V^{\lambda}>0 \text { on } \mathbb{R}^{d} .
$$

Recall $V^{\infty}(x)=\mathbb{N}_{0}\left(L^{x}>0\right)$. Let $\lambda \uparrow \infty$ in (1.25) and (1.26) to see that $V^{\lambda}(x) \uparrow V^{\infty}(x)$ and

$$
\mathbb{P}_{X_{0}}\left(L^{x}=0\right)=\exp \left(-\int \mathbb{N}_{y}\left(L^{x}>0\right) X_{0}(d y)\right)=\exp \left(-\int V^{\infty}(x-y) X_{0}(d y)\right) .
$$

It is explicitly known that (see, e.g., (2.17) in [19])

$$
V^{\infty}(x)=\frac{2(4-d)}{|x|^{2}}:=\lambda_{d}|x|^{-2},
$$

and in particular $V^{\infty}$ solves

$$
\frac{\Delta V^{\infty}}{2}=\frac{\left(V^{\infty}\right)^{2}}{2} \text { for } x \neq 0 .
$$

Write $f(t) \sim g(t)$ as $t \downarrow 0$ iff $f(t) / g(t)$ is bounded below and above by constants $c, c^{\prime}>0$ for small positive $t$, and similarly for $f(t) \sim g(t)$ as $t \rightarrow \infty$. By an application of Tauberian theorem, it is shown in Theorem 1.3 of [19] that for any $x \neq 0$,

$$
\mathbb{P}_{\delta_{0}}\left(0<L^{x}<\frac{1}{\lambda}\right) \sim V^{\infty}(x)-V^{\lambda}(x) \sim|x|^{-p} \lambda^{-\alpha} \text { as } \lambda \rightarrow \infty .
$$

The above bounds justify our explicit construction of $\mathcal{L}^{\lambda}$ in some way-one can check that as $\lambda$ is getting larger and larger, $\mathcal{L}^{\lambda}$ will concentrate more and more on the set of points $x$ whose local time $L^{x}$ is approximately $1 / \lambda$ and this probability is of order $\lambda^{-\alpha}$ by (1.30). In the end as $\lambda \rightarrow \infty$ the limiting measure will be supported on $F$ or $\partial \mathcal{R}$.

In fact we can refine the above bounds in (1.30) to exact asymptotics.

Proposition 1.12. There is some constant $c_{1.12}>0$ so that for all $x \neq 0$, we have

$$
\begin{aligned}
& \text { (i) } \lim _{\lambda \rightarrow \infty} \lambda^{\alpha} \mathbb{N}_{0}\left(0<L^{x}<1 / \lambda\right)=c_{1.12}|x|^{-p} . \\
& \text { (ii) } \lim _{\lambda \rightarrow \infty} \lambda^{\alpha} \mathbb{P}_{\delta_{0}}\left(0<L^{x}<1 / \lambda\right)=c_{1.12}|x|^{-p} e^{-V^{\infty}(x)} .
\end{aligned}
$$


The above exact asymptotic results may allow us to get an insight of the Minkowski content of $\partial \mathcal{R}$.

Conjecture 1. There is some constant $c_{1}=c_{1.12} K_{1.8}^{-1}>0$ such that

$$
\lambda^{\alpha} 1_{\left\{0<L^{x}<1 / \lambda\right\}} d x \stackrel{P}{\rightarrow} c_{1} \mathcal{L} \text { as } \lambda \rightarrow \infty \text { under } \mathbb{N}_{0} \text { and } \mathbb{P}_{\delta_{0}} .
$$

Recall $\alpha=(p-2) /(4-d)$. By an application of the improved $4-d-\eta$ Hölder continuity of $L^{x}$ for $x$ near $\partial \mathcal{R}$ for any $\eta>0$ (see [7]), we further conjecture that

Conjecture 2. There is some constant $c_{2}>0$ such that

$$
\lambda^{p-2} 1_{\{d(x, \partial \mathcal{R}) \leq 1 / \lambda\}} d x \stackrel{P}{\rightarrow} c_{2} \mathcal{L} \text { as } \lambda \rightarrow \infty \text { under } \mathbb{N}_{0} \text { and } \mathbb{P}_{\delta_{0}},
$$

which gives our conjecture on the Minkowski content of $\partial \mathcal{R}$ :

\section{Conjecture 3.}

$$
\operatorname{Cont}_{d+2-p}(\partial \mathcal{R})=c_{2} \mathcal{L}(1), \mathbb{N}_{0} \text {-a.e. and } \mathbb{P}_{\delta_{0}} \text {-a.s. }
$$

Here $\operatorname{Cont}_{\delta}(A)$ is the $\delta$-dimensional Minkowski content of any compact set $A \subset \mathbb{R}^{d}$ defined by $\operatorname{Cont}_{\delta}(A)=\lim _{r \rightarrow \infty} r^{(d-\delta)}\left|A^{\leq 1 / r}\right|$, provided the limit exists. Here we use $|\cdot|$ to denote the $d$-dimensional volume (Lebesgue measure) in $\mathbb{R}^{d}$.

\subsection{An alternate model}

While it is easy to derive from the definition of $\mathcal{L}^{\lambda}$ that the limiting measure $\mathcal{L}$ will be supported on $F$, it is not obvious that its support is actually on the smaller set $\partial \mathcal{R}$. To handle this issue we will construct another random measure $\widetilde{\mathcal{L}}(\kappa)$ supported on $\partial \mathcal{R}$ for any $\kappa>0$ by utilizing exit measures and show that there is some constant $c(\kappa)>0$ such that $\mathcal{L}=c(\kappa) \widetilde{\mathcal{L}}(\kappa)$ a.s., thus proving that $\mathcal{L}$ indeed lives on $\partial \mathcal{R}$. We also feel that the construction of $\widetilde{\mathcal{L}}(\kappa)$ may be of independent interest, given the central role exit measures have played in the study of the boundaries of the range. We first introduce the definition of exit measure. For $K_{1}, K_{2}$ non-empty, set $d\left(K_{1}, K_{2}\right)=\inf \left\{|x-y|: x \in K_{1}, y \in K_{2}\right\}$. Define

$\mathcal{O}_{X_{0}} \equiv\left\{D: D\right.$ is an open set of $\mathbb{R}^{d}$ such that $d\left(D^{c}, S\left(X_{0}\right)\right)>0$ and that, with probability one, a Brownian path starting from any $x \in \partial D$ will exit $D$ immediately\}.

In what follows we always assume that $G \in \mathcal{O}_{X_{0}}$. The exit measure of SBM $X$ from an open set $G$ under $\mathbb{P}_{X_{0}}$ and $\mathbb{N}_{X_{0}}$ is denoted by $X_{G}$ (see Chp. V of [16] for the construction of the exit measure). Intuitively $X_{G}$ is a random finite measure supported on $\partial G$, which corresponds to the mass started at $X_{0}$ which is stopped at the instant it leaves $G$. What follows may be found in Chp. V of [16] (see also Section 1 of [9]). The Laplace functional of $X_{G}$ is given by

$$
\mathbb{E}_{X_{0}}\left(\exp \left(-X_{G}(g)\right)\right)=\exp \left(-\mathbb{N}_{X_{0}}\left(1-e^{-X_{G}(g)}\right)\right)=\exp \left(-X_{0}\left(U^{g}\right)\right),
$$

where $g: \partial G \rightarrow[0, \infty)$ is continuous and $U^{g} \geq 0$ is the unique continuous function on $\bar{G}$ which is $C^{2}$ on $G$ and solves

$$
\Delta U^{g}=\left(U^{g}\right)^{2} \text { on } G, \quad U^{g}=g \text { on } \partial G .
$$

Define $G_{\varepsilon}^{x_{0}}=G_{\varepsilon}\left(x_{0}\right)=\left\{x:\left|x-x_{0}\right|>\varepsilon\right\}$ and set $G_{\varepsilon}=G_{\varepsilon}(0)$. For $\varepsilon>0$ and $\lambda \geq 0$, we let $U^{\lambda, \varepsilon}$ denote the unique continuous function on $\{|x| \geq \varepsilon\}$ such that (cf. (1.36))

$$
\Delta U^{\lambda, \varepsilon}=\left(U^{\lambda, \varepsilon}\right)^{2} \text { for }|x|>\varepsilon, \text { and } U^{\lambda, \varepsilon}(x)=\lambda \text { for }|x|=\varepsilon .
$$


Uniqueness of solutions implies the scaling property (see (3.3) of [19])

$$
U^{\lambda, \varepsilon}(x)=\varepsilon^{-2} U^{\lambda \varepsilon^{2}, 1}(x / \varepsilon) \text { for all }|x| \geq \varepsilon,
$$

and also shows $U^{\lambda, \varepsilon}$ is radially symmetric, thus allowing us to write $U^{\lambda, \varepsilon}(|x|)$ for the value at $x \in \mathbb{R}^{d}$. By (1.35) we have for any $X_{0} \in M_{F}\left(\mathbb{R}^{d}\right)$ satisfying $S\left(X_{0}\right) \subset G_{\varepsilon}$,

$$
\mathbb{E}_{X_{0}}\left(\exp \left(-\lambda X_{G_{\varepsilon}}(1)\right)\right)=\exp \left(-\mathbb{N}_{X_{0}}\left(1-e^{-\lambda X_{G_{\varepsilon}}(1)}\right)\right)=\exp \left(-X_{0}\left(U^{\lambda, \varepsilon}\right)\right) .
$$

Let $\lambda \uparrow \infty$ in the above to see that $U^{\lambda, \varepsilon} \uparrow U^{\infty, \varepsilon}$ on $G_{\varepsilon}$ and

$$
\mathbb{P}_{X_{0}}\left(X_{G_{\varepsilon}}(1)=0\right)=\exp \left(-X_{0}\left(U^{\infty, \varepsilon}\right)\right) .
$$

Proposition V.9(iii) of [16] readily implies (see also (3.5) and (3.6) of [19])

$$
\begin{aligned}
& U^{\infty, \varepsilon} \text { is } C^{2} \text { and } \Delta U^{\infty, \varepsilon}=\left(U^{\infty, \varepsilon}\right)^{2} \text { on } G_{\varepsilon}, \\
& \qquad \lim _{|x| \rightarrow \varepsilon,|x|>\varepsilon} U^{\infty, \varepsilon}(x)=+\infty, \lim _{|x| \rightarrow \infty} U^{\infty, \varepsilon}(x)=0 .
\end{aligned}
$$

Theorem 1.1 of [8] gives a construction of the local time $L^{x}$ in terms of the local asymptotic behavior of the exit measures at $x$. If $\psi_{0}(\varepsilon)=\pi^{-1} \log ^{+}(1 / \varepsilon)$ in $d=2$ and $\psi_{0}(\varepsilon)=1 /(2 \pi \varepsilon)$ in $d=3$, then for any $x \neq 0$, we have

$$
X_{G_{\varepsilon}^{x}}(1) \psi_{0}(\varepsilon) \rightarrow L^{x} \text { in measure under } \mathbb{N}_{0} \text { and } \mathbb{P}_{\delta_{0}} \text { as } \varepsilon \downarrow 0 .
$$

Motivated by the above, for any $\kappa, \varepsilon>0$, under $\mathbb{P}_{\delta_{0}}$ and $\mathbb{N}_{0}$ we define a measure $\widetilde{\mathcal{L}}(\kappa)^{\varepsilon}$ by

$$
d \widetilde{\mathcal{L}}(\kappa)^{\varepsilon}(x)=\frac{X_{G_{\varepsilon}^{x}}(1)}{\varepsilon^{p}} \exp \left(-\kappa \frac{X_{G_{\varepsilon}^{x}}(1)}{\varepsilon^{2}}\right) 1\left(X_{G_{\varepsilon / 2}^{x}}=0\right) 1(|x|>\varepsilon) d x .
$$

It is easy to derive from the definition of $X_{G_{\varepsilon}^{x}}(1)$ (see Proposition V.1 and Lemma V.2 of [16]) that for any fixed $\varepsilon>0,(\omega, x) \mapsto X_{G_{\varepsilon}^{x}}(1)(\omega)$ is $\mathcal{F} \times \mathfrak{B}\left(\mathbb{R}^{d}\right)$ measurable and so $\widetilde{\mathcal{L}}(\kappa)^{\varepsilon}$ is well defined and $\mathcal{F}$-measurable.

We can deduce from (1.42) that $\widetilde{\mathcal{L}}(\kappa)^{\varepsilon}$ is closely related to $\mathcal{L}^{\lambda}$ (as in (1.8)): for example in $d=3$, we have $\psi_{0}(\varepsilon)=1 /(2 \pi \varepsilon)$ and so $X_{G_{\varepsilon}^{x}}(1) \sim \varepsilon L^{x}$ as $\varepsilon \downarrow 0$ by (1.42). Hence if $\lambda=\kappa \varepsilon^{-1}$,

$$
\frac{X_{G_{\varepsilon}^{x}}(1)}{\varepsilon^{p}} \exp \left(-\kappa \frac{X_{G_{\varepsilon}^{x}}(1)}{\varepsilon^{2}}\right) \sim \varepsilon^{1-p} L^{x} e^{-\kappa \varepsilon^{-1} L^{x}} \sim \lambda^{1+\alpha} L^{x} e^{-\lambda L^{x}}
$$

as $\varepsilon \downarrow 0$, where in the last approximation we have used the fact that $\alpha=p-2$ in $d=3$. In (1.43), the indicator function $1(|x|>\varepsilon)$ is to ensure that $X_{G_{\varepsilon}^{x}}$ is well defined and the extra indicator $1\left(X_{G_{\varepsilon / 2}^{x}}=0\right)$ is to ensure that the limiting measures will be supported on $\partial \mathcal{R}$ rather than $F$. We will show below that they indeed differ only up to some constant.

Theorem 1.13. Let $d=2$ or 3 . For any $\kappa>0$, under both $\mathbb{N}_{0}$ and $\mathbb{P}_{\delta_{0}}$, there exists a random measure $\widetilde{\mathcal{L}}(\kappa) \in M_{F}\left(\mathbb{R}^{d}\right)$ such that $\widetilde{\mathcal{L}}(\kappa)^{\varepsilon} \stackrel{P}{\rightarrow} \widetilde{\mathcal{L}}(\kappa)$ as $\varepsilon \downarrow 0$ and there is a sequence $\varepsilon_{n} \downarrow 0$ such that $\widetilde{\mathcal{L}}(\kappa)^{\varepsilon_{n}} \rightarrow \widetilde{\mathcal{L}}(\kappa)$ a.s. as $n \rightarrow \infty$. Moreover, there is some positive constant $c_{1.13}(\kappa)$ such that $\widetilde{\mathcal{L}}(\kappa)=c_{1.13}(\kappa) \mathcal{L}$ a.s.

Turning to the $\mathbb{P}_{X_{0}}$ case, again we will restrict our interest in $S\left(X_{0}\right)^{c}$ as in (1.9). For any $\kappa, \varepsilon>0$, under $\mathbb{P}_{X_{0}}$ we define a measure $\widetilde{\mathcal{L}}(\kappa)^{\varepsilon}$ supported on $S\left(X_{0}\right)^{c}$ by

$$
d \widetilde{\mathcal{L}}(\kappa)^{\varepsilon}(x)=\frac{X_{G_{\varepsilon}^{x}}(1)}{\varepsilon^{p}} \exp \left(-\kappa \frac{X_{G_{\varepsilon}^{x}}(1)}{\varepsilon^{2}}\right) 1\left(X_{G_{\varepsilon / 2}^{x}}=0\right) 1_{\left(x \in S\left(X_{0}\right)>\varepsilon\right)} d x .
$$

Theorem 1.14. Let $d=2$ or 3 and $X_{0} \in M_{F}$. For any $\kappa>0$, under $\mathbb{P}_{X_{0}}$ there exists a $\sigma$-finite random measure $\widetilde{\mathcal{L}}(\kappa)$ such that for any $k \geq 1,\left.\left.\widetilde{\mathcal{L}}(\kappa)^{\varepsilon}\right|_{S\left(X_{0}\right) \geq 1 / k} \stackrel{P}{\rightarrow} \widetilde{\mathcal{L}}(\kappa)\right|_{S\left(X_{0}\right) \geq 1 / k}$ as $\varepsilon \downarrow 0$ and there is a sequence $\varepsilon_{n} \downarrow 0$ such that $\left.\left.\widetilde{\mathcal{L}}(\kappa)^{\varepsilon_{n}}\right|_{S\left(X_{0}\right) \geq 1 / k} \rightarrow \widetilde{\mathcal{L}}(\kappa)\right|_{S\left(X_{0}\right) \geq 1 / k}, \forall k \geq 1$ a.s. as $n \rightarrow \infty$. Moreover, we have $\widetilde{\mathcal{L}}(\kappa)=c_{1.13}(\kappa) \mathcal{L}$ a.s. 
Organization of the paper In Section 2 we give preliminary results on super-Brownian motion, the Brownian snake, exit measures and their special Markov property. In Section 3 we establish the convergence of the mean measures of $\mathcal{L}^{\lambda}$ and $\widetilde{\mathcal{L}}(\kappa)^{\varepsilon}$ and give the proof of Proposition 1.12. In Section 4 the second moment convergence results will be given in Propositions 4.1, 4.2 and 4.3 while we defer their proofs to Sections 8 and 9. Assuming the results from Section 4, we will finish the proofs of our main results Theorems 1.3 and 1.13 under $\mathbb{N}_{0}$ and $\mathbb{P}_{\delta_{0}}$ in Section 5, while we include the similar proof of Theorems 1.4 and 1.14 under $\mathbb{P}_{X_{0}}$ for general initial conditions $X_{0}$ in the Appendix A. In Section 5 we also give the proof for the first and second moment measures of $\mathcal{L}$ (Theorems 1.8 and 1.10). In Section 6 the proof of Theorem 1.6 will be finished by utilizing the shrinking ball arguments from [9]. In Section 7 a key proposition in terms of a change of measure method is given and finally in Sections 8 and 9 we finish the essential proofs of Propositions 4.1, 4.2 and 4.3.

\section{Exit measures and the special Markov property}

We will use Le Gall's Brownian snake construction of a SBM $X$, with initial condition $X_{0} \in M_{F}\left(\mathbb{R}^{d}\right)$. Set $\mathcal{W}=\cup_{t \geq 0} C\left([0, t], \mathbb{R}^{d}\right)$ with the natural metric (see page 54 of [16]), and let $\zeta(w)=t$ be the lifetime of $w \in C\left([0, t], \mathbb{R}^{d}\right) \subset \mathcal{W}$. The Brownian snake $W=\left(W_{t}, t \geq 0\right)$ is a $\mathcal{W}$-valued continuous strong Markov process and, abusing notation slightly, let $\mathbb{N}_{x}$ denote its excursion measure starting from the path at $x \in \mathbb{R}^{d}$ with lifetime zero. As usual we let $\hat{W}(t)=W_{t}\left(\zeta\left(W_{t}\right)\right)$ denote the tip of the snake at time $t$, and $\sigma(W)>0$ denote the length of the excursion path. We refer the reader to $\mathrm{Ch}$. IV of [16] for the precise definitions. The construction of super-Brownian motion, $X=X(W)$ under $\mathbb{N}_{x}$ and $\mathbb{P}_{X_{0}}$, may be found in Ch. IV of [16]. The "law" of $X(W)$ under $\mathbb{N}_{x}$ is the canonical measure of SBM starting at $x$ described in the last Section (and also denoted by $\mathbb{N}_{x}$ ). If $\Xi=\sum_{j \in J} \delta_{W_{j}}$ is a Poisson point process on $\mathcal{W}$ with intensity $\mathbb{N}_{X_{0}}(d W)=\int \mathbb{N}_{x}(d W) X_{0}(d x)$, then by Theorem 4 of Ch. IV of [16] (cf. (1.4))

$$
X_{t}(W)=\sum_{j \in J} X_{t}\left(W_{j}\right)=\int X_{t}(W) \Xi(d W) \text { for } t>0
$$

defines a SBM with initial measure $X_{0}$. We will refer to this as the standard set-up for $X$ under $\mathbb{P}_{X_{0}}$. It follows that the total local time $L^{x}$ under $\mathbb{P}_{X_{0}}$ may also be decomposed as

$$
L^{x}=\sum_{j \in J} L^{x}\left(W_{j}\right)=\int L^{x}(W) \Xi(d W) .
$$

Recall $\mathcal{R}=\overline{\left\{x: L^{x}>0\right\}}$ is the range of the SBM $X$ under $\mathbb{P}_{X_{0}}$ and $\mathbb{N}_{X_{0}}$. Under $\mathbb{N}_{X_{0}}$ we have (see (8) on p. 69 of [16])

$$
\mathcal{R}=\{\hat{W}(s): s \in[0, \sigma]\} .
$$

Let $G \in \mathcal{O}_{X_{0}}$ as in (1.34). Then

$$
X_{G} \text { is a finite random measure supported on } \mathcal{R} \cap \partial G \text { a.s. }
$$

Under $\mathbb{N}_{X_{0}}$ this follows from the definition of $X_{G}$ on p. 77 of [16] and the ensuing discussion, and (2.3). Although [16] works under $\mathbb{N}_{x}$ for $x \in G$ the above extends immediately to $\mathbb{P}_{X_{0}}$ because as in (2.23) of [19],

$$
X_{G}=\sum_{j \in J} X_{G}\left(W_{j}\right)=\int X_{G}(W) d \Xi(W),
$$


where $\Xi$ is a Poisson point process on $\mathcal{W}$ with intensity $\mathbb{N}_{X_{0}}$.

Working under $\mathbb{N}_{X_{0}}$ and following [15], we define

$$
\begin{gathered}
S_{G}\left(W_{u}\right)=\inf \left\{t \leq \zeta_{u}: W_{u}(t) \notin G\right\} \quad(\inf \emptyset=\infty), \\
\eta_{s}^{G}(W)=\inf \left\{t: \int_{0}^{t} 1\left(\zeta_{u} \leq S_{G}\left(W_{u}\right)\right) d u>s\right\}, \\
\mathcal{E}_{G}=\sigma\left(W_{\eta_{s}^{G}}, s \geq 0\right) \vee\left\{\mathbb{N}_{X_{0}}-\text { null sets }\right\},
\end{gathered}
$$

where $s \rightarrow W_{\eta_{s}^{G}}$ is continuous (see p. 401 of [15]). Write the open set $\left\{u: S_{G}\left(W_{u}\right)<\zeta_{u}\right\}$ as countable union of disjoint open intervals, $\cup_{i \in I}\left(a_{i}, b_{i}\right)$. Clearly $S_{G}\left(W_{u}\right)=S_{G}^{i}<\infty$ for all $u \in\left[a_{i}, b_{i}\right]$ and we may define

$$
W_{s}^{i}(t)=W_{\left(a_{i}+s\right) \wedge b_{i}}\left(S_{G}^{i}+t\right) \text { for } 0 \leq t \leq \zeta_{\left(a_{i}+s\right) \wedge b_{i}}-S_{G}^{i}
$$

Therefore for $i \in I, W^{i} \in C\left(\mathbb{R}_{+}, \mathcal{W}\right)$ are the excursions of $W$ outside $G$. Proposition 2.3 of [15] implies $X_{G}$ is $\mathcal{E}_{G}$-measurable and Corollary 2.8 of the same reference implies

$$
\left\{\begin{array}{l}
\text { Conditional on } \mathcal{E}_{G}, \text { the point measure } \sum_{i \in I} \delta_{W^{i}} \text { is a Poisson } \\
\text { point measure with intensity } \mathbb{N}_{X_{G}} .
\end{array}\right.
$$

If $D$ is an open set in $\mathcal{O}_{X_{0}}$ such that $\bar{G} \subset D$ and $d\left(D^{c}, \bar{G}\right)>0$, then the definition (and existence) of $X_{D}(W)$ applies equally well to each $X_{D}\left(W^{i}\right)$ and it is easy to check that

$$
X_{D}(W)=\sum_{i \in I} X_{D}\left(W^{i}\right)
$$

If $U$ is an open subset of $S\left(X_{0}\right)^{c}$, then $L_{U}$, the restriction of the local time $L^{x}$ to $U$, is in $C(U)$ which is the set of continuous functions on $U$.

Proposition 2.1. Let $X_{0} \in M_{F}\left(\mathbb{R}^{d}\right)$.

(i) Let $G$ be an open set in $\mathcal{O}_{X_{0}}$. Let $\psi_{0}$ be a bounded measurable function on $C\left(\bar{G}^{c}\right)$, $n \geq 1$ and $\Phi_{1}$ be a bounded measurable function on $M_{F}\left(\mathbb{R}^{d}\right)^{n}$. Let $D_{i}$ be open sets in $\mathcal{O}_{X_{0}}$, such that $\bar{G} \subset D_{i}$ and $d\left(D_{i}^{c}, \bar{G}\right)>0, \forall 1 \leq i \leq n$. Then

$$
\mathbb{N}_{X_{0}}\left(\psi_{0}\left(L_{\bar{G}^{c}}\right) \Phi_{1}\left(X_{D_{1}}, \ldots, X_{D_{n}}\right) \mid \mathcal{E}_{G}\right)=\mathbb{E}_{X_{G}}\left(\psi_{0}\left(L_{\bar{G}^{c}}\right) \Phi_{1}\left(X_{D_{1}}, \ldots, X_{D_{n}}\right)\right) .
$$

(ii) Let $G_{1}, G_{2}$ be open sets in $\mathcal{O}_{X_{0}}$ such that $\overline{G_{1}} \subset G_{2}$ and $d\left(G_{2}^{c}, \overline{G_{1}}\right)>0$. If $\psi_{2}: \mathcal{K} \rightarrow \mathbb{R}$ is Borel measurable, then we have

$$
\mathbb{N}_{X_{0}}\left(\psi_{2}\left(\mathcal{R} \cap G_{2}^{c}\right) \mid \mathcal{E}_{G_{1}}\right)=\mathbb{E}_{X_{G_{1}}}\left(\psi_{2}\left(\mathcal{R} \cap G_{2}^{c}\right)\right),
$$

where $\mathcal{K}$ is the space of compact subsets of $\mathbb{R}^{d}$ equipped with the Hausdorff metric (see, e.g., Section 2 of [9]).

(iii) Let $G_{1}, G_{2}$ be open sets in $\mathcal{O}_{X_{0}}$ such that $\overline{G_{1}} \subset G_{2}$ and $d\left(G_{2}^{c}, \overline{G_{1}}\right)>0$. If $\psi_{3}: \mathbb{R} \rightarrow \mathbb{R}$ is Borel measurable, then for any $\lambda>0$ we have

$$
\mathbb{N}_{X_{0}}\left(\psi_{3}\left(\mathcal{L}^{\lambda}\left(G_{2}^{c}\right)\right) \mid \mathcal{E}_{G_{1}}\right)=\mathbb{E}_{X_{G_{1}}}\left(\psi_{3}\left(\mathcal{L}^{\lambda}\left(G_{2}^{c}\right)\right)\right) .
$$

Proof. (ii) follows immediately from Proposition 2.2 in [9]. (i) and (iii) will follow in a similar way as Proposition 2.6(b) of [19].

We will need a version of the above under $\mathbb{P}_{X_{0}}$ as well, which is Proposition 2.3 in [9]. 
Proposition 2.2. Let $X_{0} \in M_{F}\left(\mathbb{R}^{d}\right)$.

(i) Let $G$ be an open set in $\mathcal{O}_{X_{0}}$. Let $\psi_{0}$ be a bounded measurable function on $C\left(\bar{G}^{c}\right)$, $n \geq 1$ and $\Phi_{1}$ be a bounded measurable function on $M_{F}\left(\mathbb{R}^{d}\right)^{n}$. Let $D_{i}$ be open sets in $\mathcal{O}_{X_{0}}$, such that $\bar{G} \subset D_{i}$ and $d\left(D_{i}^{c}, \bar{G}\right)>0, \forall 1 \leq i \leq n$. Then

$$
\mathbb{E}_{X_{0}}\left(\psi_{0}\left(L_{\bar{G}^{c}}\right) \Phi_{1}\left(X_{D_{1}}, \ldots, X_{D_{n}}\right) \mid \mathcal{E}_{G}\right)=\mathbb{E}_{X_{G}}\left(\psi_{0}\left(L_{\bar{G}^{c}}\right) \Phi_{1}\left(X_{D_{1}}, \ldots, X_{D_{n}}\right)\right) .
$$

(ii) Let $G_{1}, G_{2}$ be open sets in $\mathcal{O}_{X_{0}}$ such that $\overline{G_{1}} \subset G_{2}$ and $d\left(G_{2}^{c}, \overline{G_{1}}\right)>0$. If $\psi_{2}: \mathcal{K} \rightarrow \mathbb{R}$ is Borel measurable, then we have

$$
\mathbb{E}_{X_{0}}\left(\psi_{2}\left(\mathcal{R} \cap G_{2}^{c}\right) \mid \mathcal{E}_{G_{1}}\right)=\mathbb{E}_{X_{G_{1}}}\left(\psi_{2}\left(\mathcal{R} \cap G_{2}^{c}\right)\right)
$$

\section{Convergence of the mean measure and proof of Proposition 1.12}

In this section we will give the convergence of first moment measures of $\mathcal{L}^{\lambda}$ and $\widetilde{\mathcal{L}}(\kappa)^{\varepsilon}$ and finish the proof of Proposition 1.12 .

\subsection{Mean measure for local time}

Recall $V^{\lambda}(x)=\mathbb{N}_{0}\left(1-e^{-\lambda L^{x}}\right)$ as in (1.25) and $V^{\lambda}$ is also the solution to (1.26). Uniqueness of solutions implies the scaling property (see (2.13) of [19])

$$
V^{\lambda}(x)=r^{-2} V^{\lambda r^{4-d}}(x / r) \text { for all } x \neq 0, r>0,
$$

and also shows $V^{\lambda}$ is radially symmetric, thus allowing us to write $V^{\lambda}(|x|)$ for the value at $x \in \mathbb{R}^{d}$. The monotone convergence theorem and the convexity of $e^{-a x}$ for $a, x>0$ allow us to differentiate $V^{\lambda}(x)=\mathbb{N}_{0}\left(1-e^{-\lambda L^{x}}\right)$ with respect to $\lambda>0$ through the expectation so that for any $\lambda>0$, we can define

$$
V_{1}^{\lambda}(x):=\frac{\partial}{\partial \lambda} V^{\lambda}(x)=\mathbb{N}_{0}\left(L^{x} e^{-\lambda L^{x}}\right), \forall x \neq 0 .
$$

By differentiating both sides of (3.1) with respect to $\lambda>0$, we obtain

$$
V_{1}^{\lambda}(x)=r^{-2} V_{1}^{\lambda r^{4-d}}(x / r) r^{4-d}=r^{-2} \mathbb{N}_{0}\left(r^{4-d} L^{x / r} e^{-\lambda r^{4-d} L^{x / r}}\right),
$$

which is also a consequence of the scaling of Brownian snake under $\mathbb{N}_{0}$ (see, e.g., the proof of Proposition V.9 (i) of [16]). Before turning to the calculation of the mean measure of $\mathcal{L}^{\lambda}$, we recall $\alpha$ as in (1.3) and give the following result from Proposition 5.5 of [19].

Lemma 3.1. There is some constant $c_{3.1}>0$, depending on $d$ so that

$$
V^{\infty}(x)-V^{\lambda}(x) \leq c_{3.1}|x|^{-p} \lambda^{-\alpha}, \forall x \neq 0, \lambda>0 .
$$

The following is an easy consequence of the above lemma.

Proposition 3.2. There is some constant $c_{3.2}>0$, depending on $d$ so that

$$
\mathbb{N}_{0}\left(\lambda^{1+\alpha} L^{x} e^{-\lambda L^{x}}\right)=\lambda^{1+\alpha} V_{1}^{\lambda}(x) \leq c_{3.2}|x|^{-p}, \forall x \neq 0, \lambda>0 .
$$

Proof. The first equality is immediate by definition (3.2). One can also conclude from (3.2) that $\lambda \mapsto V_{1}^{\lambda}(x)$ is monotone decreasing and so for any $\lambda>0$,

$$
\begin{aligned}
V_{1}^{\lambda}(x) & \leq \frac{2}{\lambda} \int_{\lambda / 2}^{\lambda} V_{1}^{\lambda^{\prime}}(x) d \lambda^{\prime}=\frac{2}{\lambda}\left(V^{\lambda}(x)-V^{\lambda / 2}(x)\right) \\
& \leq \frac{2}{\lambda}\left(V^{\infty}(x)-V^{\lambda / 2}(x)\right) \leq \frac{2}{\lambda} c_{3.1}|x|^{-p} \lambda^{-\alpha} 2^{\alpha},
\end{aligned}
$$

where the last inequality is from Lemma 3.1. Let $c_{3.2}=2^{1+\alpha} c_{3.1}$ to finish the proof. 
Let $\left(B_{t}\right)$ denote a $d$-dimentional Brownian motion starting from $x$ under $P_{x}$. Define $\tau_{r}=\inf \left\{t \geq 0:\left|B_{t}\right| \leq r\right\}$ for any $r>0$ and let $r_{\lambda}=\lambda_{0} \lambda^{-\frac{1}{4-d}}$ where $\lambda_{0}$ will be chosen to be some fixed large constant below. In what follows we will always assume $0<r_{\lambda}<|x|$.

Lemma 3.3. Let $\lambda>0$ and $|x|>r_{\lambda}>0$. For any $t>0$, we have

$$
V_{1}^{\lambda}(x)=E_{x}\left(V_{1}^{\lambda}\left(B\left(t \wedge \tau_{r_{\lambda}}\right)\right) \exp \left(-\int_{0}^{t \wedge \tau_{r_{\lambda}}} V^{\lambda}\left(B_{s}\right) d s\right)\right) .
$$

Proof. It follows in a similar way to that of Lemma 9.4 in [19].

For $\gamma \in \mathbb{R}$, we let $\left(\rho_{t}\right)$ denote a $\gamma$-dimensional Bessel process starting from $r>0$ under $P_{r}^{(\gamma)}$ and let $\left(\mathcal{F}_{t}^{\rho}\right)$ denote the right-continuous filtration generated by the Bessel process. We slightly abuse the notation and define $\tau_{R}=\tau_{R}^{\rho}=\inf \left\{t \geq 0: \rho_{t} \leq R\right\}$ for $R>0$. The following results (i) and (ii) are from Lemmas 5.2 and 5.3 of [19] and the last one follows from (ii) and a simple application of the Cauchy-Schwartz inequality.

Lemma 3.4. Assume $0<2 \gamma \leq \nu^{2}$ and $q>2$. Then

(i)

$$
E_{r}^{(2+2 \nu)}\left(\exp \left(\int_{0}^{\tau_{1}} \frac{\gamma}{\rho_{s}^{2}} d s\right) \mid \tau_{1}<\infty\right)=r^{\nu-\sqrt{\nu^{2}-2 \gamma}}, \forall r \geq 1
$$

$\sup _{r \geq 1} E_{r}^{(2+2 \nu)}\left(\exp \left(\int_{0}^{\tau_{1}} \frac{\gamma}{\rho_{s}^{q}} d s\right) \mid \tau_{1}<\infty\right) \leq C_{3.4}(q, \nu)<\infty$.

$$
\inf _{r \geq 1} E_{r}^{(2+2 \nu)}\left(\exp \left(-\int_{0}^{\tau_{1}} \frac{\gamma}{\rho_{s}^{q}} d s\right) \mid \tau_{1}<\infty\right) \geq c_{3.4}(q, \nu)>0 .
$$

Lemma 3.5. Let $r_{\lambda}=\lambda_{0} \lambda^{-\frac{1}{4-d}}$. There is some constant $c_{3.5}>0$ such that for all $\lambda_{0}>c_{3.5}$, $0<\gamma \leq 2$, there is some constant $C_{3.5}\left(\lambda_{0}, \nu, \gamma\right)>0$ so that for all $x \neq 0$,

$$
\begin{aligned}
& \sup _{\lambda>0} E_{|x|}^{(2+2 \nu)}\left(\exp \left(\gamma \int_{0}^{\tau_{r_{\lambda}}}\left(V^{\infty}-V^{\lambda}\right)\left(\rho_{s}\right) d s\right) \mid \tau_{r_{\lambda}}<\infty\right) \\
& =\lim _{\lambda \rightarrow \infty} E_{|x|}^{(2+2 \nu)}\left(\exp \left(\gamma \int_{0}^{\tau_{r_{\lambda}}}\left(V^{\infty}-V^{\lambda}\right)\left(\rho_{s}\right) d s\right) \mid \tau_{r_{\lambda}}<\infty\right) \\
& =C_{3.5}\left(\lambda_{0}, \nu, \gamma\right)<\infty .
\end{aligned}
$$

Proof. The scalings of Bessel process $\rho_{s}$ and $V^{\infty}, V^{\lambda}$ give us that

$$
\begin{aligned}
& E_{|x|}^{(2+2 \nu)}\left(\exp \left(\gamma \int_{0}^{\tau_{r_{\lambda}}}\left(V^{\infty}-V^{\lambda}\right)\left(\rho_{s}\right) d s\right) \mid \tau_{r_{\lambda}}<\infty\right) \\
= & E_{|x| / r_{\lambda}}^{(2+2 \nu)}\left(\exp \left(\gamma \int_{0}^{\tau_{1}}\left(V^{\infty}-V^{\lambda r_{\lambda}^{4-d}}\right)\left(\rho_{s}\right) d s\right) \mid \tau_{1}<\infty\right) \\
= & E_{|x| / r_{\lambda}}^{(2+2 \nu)}\left(\exp \left(\gamma \int_{0}^{\tau_{1}}\left(V^{\infty}-V^{\lambda_{0}^{4-d}}\right)\left(\rho_{s}\right) d s\right) \mid \tau_{1}<\infty\right),
\end{aligned}
$$

where we have used $r_{\lambda}=\lambda_{0} \lambda^{-\frac{1}{4-d}}$ in the last line. For any $r>1$, we let

$$
\begin{aligned}
f(r) & :=E_{r}^{(2+2 \nu)}\left(\exp \left(\gamma \int_{0}^{\tau_{1}}\left(V^{\infty}-V^{\lambda_{0}^{4-d}}\right)\left(\rho_{s}\right) d s\right) \mid \tau_{1}<\infty\right) \\
& =E_{r}^{(2+2 \nu)}\left(\exp \left(\gamma \int_{0}^{\tau_{1}}\left(V^{\infty}-V^{\lambda_{0}^{4-d}}\right)\left(\rho_{s}\right) d s\right) 1\left(\tau_{1}<\infty\right)\right) r^{2 \nu}
\end{aligned}
$$

where the second line is by $P_{r}^{(2+2 \nu)}\left(\tau_{R}<\infty\right)=(R / r)^{2 \nu}$ for any $r>R>0$. By (3.7) and the definition of $r_{\lambda}$, it suffices to show that there is some constant $C_{3.5}\left(\lambda_{0}, \nu, \gamma\right)>0$ so that $\sup _{r>1} f(r)=\lim _{r \rightarrow \infty} f(r)=C_{3.5}\left(\lambda_{0}, \nu, \gamma\right)$. 
Let $r>R>1$ and apply the strong Markov property in (3.8) to get

$$
\begin{aligned}
& f(r)=E_{r}^{(2+2 \nu)}\left(\exp \left(\gamma \int_{0}^{\tau_{R}}\left(V^{\infty}-V^{\lambda_{0}^{4-d}}\right)\left(\rho_{s}\right) d s\right) 1\left(\tau_{R}<\infty\right)\right) \\
& E_{R}^{(2+2 \nu)}\left(\exp \left(\gamma \int_{0}^{\tau_{1}}\left(V^{\infty}-V^{\lambda_{0}^{4-d}}\right)\left(\rho_{s}\right) d s\right) 1\left(\tau_{1}<\infty\right)\right) r^{2 \nu} \\
&=E_{r}^{(2+2 \nu)}\left(\exp \left(\gamma \int_{0}^{\tau_{R}}\left(V^{\infty}-V^{\lambda_{0}^{4-d}}\right)\left(\rho_{s}\right) d s\right) \mid \tau_{R}<\infty\right) \\
& E_{R}^{(2+2 \nu)}\left(\exp \left(\gamma \int_{0}^{\tau_{1}}\left(V^{\infty}-V^{\lambda_{0}^{4-d}}\right)\left(\rho_{s}\right) d s\right) \mid \tau_{1}<\infty\right) \geq f(R),
\end{aligned}
$$

and it follows that $r \mapsto f(r)$ is monotone increasing for $r>1$. By using Lemma 3.1 and Lemma 3.4 (ii), we have

$$
\sup _{r \geq 1} f(r) \leq \sup _{r \geq 1} E_{r}^{(2+2 \nu)}\left(\exp \left(\int_{0}^{\tau_{1}} c_{3.1} \gamma \lambda_{0}^{-(p-2)} \rho_{s}^{-p} d s\right) \mid \tau_{1}<\infty\right)<\infty,
$$

if we choose $\lambda_{0}$ large enough so that $2 \gamma c_{3.1} \lambda_{0}^{-(p-2)} \leq 4 c_{3.1} \lambda_{0}^{-(p-2)}<\nu^{2}$. Hence we conclude $\sup _{r \geq 1} f(r)=\lim _{r \rightarrow \infty} f(r)=C_{3.5}\left(\lambda_{0}, \nu, \gamma\right)$ for some constant $C_{3.5}\left(\lambda_{0}, \nu, \gamma\right)>0$ and the proof is complete as noted above.

We also state a result on the application of Girsanov's theorem on Bessel process from [24] (see also Proposition 2.5 of [19]).

Lemma 3.6. Let $\lambda \geq 0, \mu \in \mathbb{R}, r>0$ and $\nu=\sqrt{\lambda^{2}+\mu^{2}}$. If $\Phi_{t} \geq 0$ is $\mathcal{F}_{t}^{\rho}$-adapted, then for all $R<r$, we have

$$
E_{r}^{(2+2 \mu)}\left(\Phi_{t \wedge \tau_{R}} \exp \left(-\frac{\lambda^{2}}{2} \int_{0}^{t \wedge \tau_{R}} \frac{1}{\rho_{s}^{2}} d s\right)\right)=r^{\nu-\mu} E_{r}^{(2+2 \nu)}\left(\left(\rho_{t \wedge \tau_{R}}\right)^{-\nu+\mu} \Phi_{t \wedge \tau_{R}}\right) .
$$

The following result is an easy application of the above lemma and is proved in Proposition 4.3 of [8].

Proposition 3.7. Let $x \in \mathbb{R}^{d} \backslash\{0\}$ and $0<\varepsilon<|x|$. For any Borel measurable function $g:(0, \infty) \rightarrow \mathbb{R}$ that is bounded on any compact subset of $(0, \infty)$, we have

$$
\begin{aligned}
& E_{x}\left(1\left(\tau_{\varepsilon}<\infty\right) \exp \left(-\int_{0}^{\tau_{\varepsilon}} g\left(\left|B_{s}\right|\right) d s\right)\right) \\
& \quad=\varepsilon^{p}|x|^{-p} E_{|x|}^{(2+2 \nu)}\left(\exp \left(-\int_{0}^{\tau_{\varepsilon}}\left(g\left(\rho_{s}\right)-V^{\infty}\left(\rho_{s}\right)\right) d s\right) \mid \tau_{\varepsilon}<\infty\right),
\end{aligned}
$$

where $B$ is a $d$-dimensional Brownian motion under $P_{x}$ for $d \leq 3$ and $\nu$ is as in (1.13).

Proposition 3.8. There is some constant $c_{3.8}=c_{3.8}(d)>0$ such that

$$
\lim _{\lambda \rightarrow \infty} \mathbb{N}_{0}\left(\lambda^{1+\alpha} L^{x} e^{-\lambda L^{x}}\right)=\lim _{\lambda \rightarrow \infty} \lambda^{1+\alpha} V_{1}^{\lambda}(x)=c_{3.8}|x|^{-p}, \forall x \neq 0 .
$$

Proof. Recall $r_{\lambda}=\lambda_{0} \lambda^{-\frac{1}{4-d}}$. We use Lemma 3.3, and the facts that $V_{1}^{\lambda}(x) \rightarrow 0$ as $|x| \rightarrow \infty$ and $V_{1}^{\lambda}\left(B_{t \wedge \tau_{r_{\lambda}}}\right)$ is uniformly bounded for all $t \geq 0$ by Proposition 3.2 , to see that

$$
\begin{aligned}
\lambda^{1+\alpha} V_{1}^{\lambda}(x) & =\lambda^{1+\alpha} \lim _{t \rightarrow \infty} E_{x}\left(V_{1}^{\lambda}\left(B_{t \wedge \tau_{r_{\lambda}}}\right) \exp \left(-\int_{0}^{t \wedge \tau_{r_{\lambda}}} V^{\lambda}\left(B_{s}\right) d s\right)\right) \\
& =\lambda^{1+\alpha} E_{x}\left(1\left(\tau_{r_{\lambda}}<\infty\right) V_{1}^{\lambda}\left(B_{\tau_{r_{\lambda}}}\right) \exp \left(-\int_{0}^{\tau_{r_{\lambda}}} V^{\lambda}\left(B_{s}\right) d s\right)\right) \\
& =\lambda^{1+\alpha} V_{1}^{\lambda}\left(r_{\lambda}\right) E_{x}\left(1\left(\tau_{r_{\lambda}}<\infty\right) \exp \left(-\int_{0}^{\tau_{r_{\lambda}}} V^{\lambda}\left(\left|B_{s}\right|\right) d s\right)\right) \\
& =\lambda^{1+\alpha} V_{1}^{\lambda}\left(r_{\lambda}\right) \frac{r_{\lambda}^{p}}{|x|^{p}} E_{|x|}^{(2+2 \nu)}\left(\exp \left(\int_{0}^{\tau_{r_{\lambda}}}\left(V^{\infty}-V^{\lambda}\right)\left(\rho_{s}\right) d s\right) \mid \tau_{r_{\lambda}}<\infty\right)
\end{aligned}
$$


where the third equality is by the radial symmetry of $V^{\lambda}$ and $V_{1}^{\lambda}$. The last equality follows from Proposition 3.7 with $g=V^{\lambda}$. Use the scaling of $V_{1}^{\lambda}$ from (3.3) to see that the right-hand side of the above equals

$$
\begin{aligned}
& \lambda^{1+\alpha} r_{\lambda}^{2-d} V_{1}^{\lambda r_{\lambda}^{4-d}}(1) \frac{r_{\lambda}^{p}}{|x|^{p}} E_{|x|}^{(2+2 \nu)}\left(\exp \left(\int_{0}^{\tau_{r_{\lambda}}}\left(V^{\infty}-V^{\lambda}\right)\left(\rho_{s}\right) d s\right) \mid \tau_{r_{\lambda}}<\infty\right) \\
& =|x|^{-p} \lambda_{0}^{p+2-d} V_{1}^{\lambda_{0}^{4-d}}(1) E_{|x|}^{(2+2 \nu)}\left(\exp \left(\int_{0}^{\tau_{r_{\lambda}}}\left(V^{\infty}-V^{\lambda}\right)\left(\rho_{s}\right) d s\right) \mid \tau_{r_{\lambda}}<\infty\right),
\end{aligned}
$$

where we have used the definition of $r_{\lambda}$ and $\alpha$ in the last equality. Choose $\lambda_{0}>c_{3.5}$ and then apply Lemma 3.5 with $\gamma=1$ to conclude

$$
\lim _{\lambda \rightarrow \infty} \lambda^{1+\alpha} V_{1}^{\lambda}(x)=\lambda_{0}^{p+2-d} V_{1}^{\lambda_{0}^{4-d}}(1) C_{3.5}\left(\lambda_{0}, \nu, 1\right)|x|^{-p},
$$

and so the proof is complete.

Corollary 3.9. For any $x \in S\left(X_{0}\right)^{c}$ we have

$$
\lim _{\lambda \rightarrow \infty} \mathbb{E}_{X_{0}}\left(\lambda^{1+\alpha} L^{x} e^{-\lambda L^{x}}\right)=e^{-\int V^{\infty}(y-x) X_{0}(d y)} c_{3.8} \int|y-x|^{-p} X_{0}(d y) .
$$

Proof. For any $x \in S\left(X_{0}\right)^{c}$, we have $d\left(x, S\left(X_{0}\right)\right)>0$. The monotone convergence theorem and the convexity of $e^{-a x}$ for $a, x>0$ allow us to differentiate (1.39) to get

$$
\mathbb{E}_{X_{0}}\left(L^{x} e^{-\lambda L^{x}}\right)=\int V_{1}^{\lambda}(y-x) X_{0}(d y) e^{-\int V^{\lambda}(y-x) X_{0}(d y)} .
$$

By Proposition 3.2 we have $\lambda^{1+\alpha} V_{1}^{\lambda}(y-x) \leq c_{3.2}|y-x|^{-p}, \forall y \neq x, \lambda>0$, and so by Proposition 3.8 we may apply the dominated convergence theorem to get

$$
\lim _{\lambda \rightarrow \infty} \int \lambda^{1+\alpha} V_{1}^{\lambda}(y-x) X_{0}(d y)=\int c_{3.8}|y-x|^{-p} X_{0}(d y) .
$$

Then it follows easily from (3.12), (3.13) and the monotone convergence theorem.

\subsection{Left tail of the local time}

Proof of Proposition 1.12. First recall $V^{\lambda}$ and $V^{\infty}$ from (1.25) and (1.27) to see that for all $|x|>0$, we have

$$
\lambda^{\alpha} \mathbb{N}_{0}\left(e^{-\lambda L^{x}} 1\left(L^{x}>0\right)\right)=\lambda^{\alpha}\left(V^{\infty}(x)-V^{\lambda}(x)\right) .
$$

Let $d^{\lambda}(x)=V^{\infty}(x)-V^{\lambda}(x)$ and $r_{\lambda}$ be as in Lemma 3.3. By the Feyman-Kac formula for $d^{\lambda}$ (as in (5.2) of [19]), we get for $|x|>r_{\lambda}>0$,

$$
d^{\lambda}(x)=d^{\lambda}\left(r_{\lambda}\right) E_{x}\left(1_{\left\{\tau_{r_{\lambda}}<\infty\right\}} \exp \left(-\int_{0}^{\tau_{r_{\lambda}}} \frac{\left(V^{\infty}+V^{\lambda}\right)\left(B_{s}\right)}{2} d s\right)\right) .
$$

By the scaling of $V^{\lambda}$ and $V^{\infty}$ and the definition of $r_{\lambda}$, we have

$$
d^{\lambda}\left(r_{\lambda}\right)=\left(V^{\infty}-V^{\lambda}\right)\left(r_{\lambda}\right)=r_{\lambda}^{-2}\left(V^{\infty}(1)-V^{\lambda_{0}^{4-d}}(1)\right)=r_{\lambda}^{-2} d^{\lambda_{0}^{4-d}}(1) .
$$

Use the above and (3.15) to see that

$$
\begin{aligned}
& \lambda^{\alpha} d^{\lambda}(x)=\lambda^{\alpha} r_{\lambda}^{-2} d^{\lambda_{0}^{4-d}}(1) E_{x}\left(1_{\left\{\tau_{r_{\lambda}}<\infty\right\}} \exp \left(-\int_{0}^{\tau_{r_{\lambda}}} \frac{\left(V^{\infty}+V^{\lambda}\right)\left(B_{s}\right)}{2} d s\right)\right) \\
= & \lambda^{\alpha} r_{\lambda}^{-2} d^{\lambda_{0}^{4-d}}(1) r_{\lambda}^{p}|x|^{-p} E_{|x|}^{(2+2 \nu)}\left(\exp \left(\int_{0}^{\tau_{r_{\lambda}}} \frac{\left(V^{\infty}-V^{\lambda}\right)\left(\rho_{s}\right)}{2} d s\right) \mid \tau_{r_{\lambda}}<\infty\right) \\
= & |x|^{-p} d^{\lambda_{0}^{4-d}}(1) \lambda_{0}^{p-2} E_{|x|}^{(2+2 \nu)}\left(\exp \left(\int_{0}^{\tau_{r_{\lambda}}} \frac{\left(V^{\infty}-V^{\lambda}\right)\left(\rho_{s}\right)}{2} d s\right) \mid \tau_{r_{\lambda}}<\infty\right),
\end{aligned}
$$


where the second equality is by Proposition 3.7 and in the last equality we have used the definitions of $r_{\lambda}$ and $\alpha$. Choose $\lambda_{0}>c_{3.5}$ so that we can apply Lemma 3.5 with $\gamma=1 / 2$ to get

$$
\lim _{\lambda \rightarrow \infty} \lambda^{\alpha} d^{\lambda}(x)=|x|^{-p} d^{\lambda_{0}^{4-d}}(1) \lambda_{0}^{p-2} C_{3.5}\left(\lambda_{0}, \nu, 1 / 2\right) .
$$

Recalling (3.14), we apply Tauberian theorem (see, e.g., Theorem 5.1 and 5.3 of Chp. XIII of [3]) to get

$$
\lim _{\lambda \rightarrow \infty} \lambda^{\alpha} \mathbb{N}_{0}\left(0<L^{x}<1 / \lambda\right)=c_{1.12}|x|^{-p},
$$

where $c_{1.12}=(\Gamma(\alpha+1))^{-1} d^{\lambda_{0}^{4-d}}(1) \lambda_{0}^{p-2} C_{3.5}\left(\lambda_{0}, \nu, 1 / 2\right)$ and the proof of (i) is complete. Turning to (ii) for $\mathbb{P}_{\delta_{0}}$, we note that for all $|x|>0$, by (1.25) and (1.27) we have

$$
\begin{aligned}
& \lambda^{\alpha} \mathbb{E}_{\delta_{0}}\left(e^{-\lambda L^{x}} 1_{\left(L^{x}>0\right)}\right) \\
& =\lambda^{\alpha}\left(e^{-V^{\lambda}(x)}-e^{-V^{\infty}(x)}\right)=\lambda^{\alpha} e^{-V^{\infty}(x)}\left(e^{V^{\infty}(x)-V^{\lambda}(x)}-1\right) \\
& \rightarrow e^{-V^{\infty}(x)}|x|^{-p} d^{\lambda_{0}^{4-d}}(1) \lambda_{0}^{p-2} C_{3.5}\left(\lambda_{0}, \nu, 1 / 2\right) \text { as } \lambda \rightarrow \infty,
\end{aligned}
$$

where the last line follows from (3.16). Apply Tauberian theorem again to get (ii) and the proof is complete.

\subsection{Mean measure for exit measure}

Now we will turn to the alternate model using exit measures. Recall from (1.39) that

$$
U^{\lambda \varepsilon^{-2}, \varepsilon}(x)=\mathbb{N}_{0}\left(1-\exp \left(-\lambda \frac{X_{G_{\varepsilon}^{x}}(1)}{\varepsilon^{2}}\right)\right), \forall|x|>\varepsilon .
$$

Similar to (3.2), we can differentiate the above with respect to $\lambda>0$ through the expectation so that for any $\lambda>0$ and for all $|x|>\varepsilon$, we have

$$
U_{1}^{\lambda \varepsilon^{-2}, \varepsilon}(x):=\frac{\partial}{\partial \lambda} U^{\lambda \varepsilon^{-2}, \varepsilon}(x)=\mathbb{N}_{0}\left(\frac{X_{G_{\varepsilon}^{x}}(1)}{\varepsilon^{2}} \exp \left(-\lambda \frac{X_{G_{\varepsilon}^{x}}(1)}{\varepsilon^{2}}\right)\right) .
$$

By using Proposition 2.1(i), for any $|x|>\varepsilon>0$ we have (more details can be found in the derivation of (4.2) in [8])

$$
\begin{aligned}
& \mathbb{N}_{0}\left(\frac{X_{G_{\varepsilon}^{x}}(1)}{\varepsilon^{p}} \exp \left(-\kappa \frac{X_{G_{\varepsilon}^{x}}(1)}{\varepsilon^{2}}\right) 1\left(X_{G_{\varepsilon / 2}^{x}}=0\right)\right) \\
= & \mathbb{N}_{0}\left(\frac{X_{G_{\varepsilon}^{x}}(1)}{\varepsilon^{p}} \exp \left(-\left(\kappa+4 U^{\infty, 1}(2)\right) \frac{X_{G_{\varepsilon}^{x}}(1)}{\varepsilon^{2}}\right)\right) .
\end{aligned}
$$

The following result on the convergence of the mean measure of $\widetilde{\mathcal{L}}(\kappa)$ is proved in Theorem 1.3 of [8].

Proposition 3.10. For any $\kappa>0$, there is some constant $C_{3.10}(\kappa)>0$ such that for all $x \neq 0$,

$$
\lim _{\varepsilon \downarrow 0} \mathbb{N}_{0}\left(\frac{X_{G_{\varepsilon}^{x}}(1)}{\varepsilon^{p}} \exp \left(-\kappa \frac{X_{G_{\varepsilon}^{x}}(1)}{\varepsilon^{2}}\right) 1\left(X_{G_{\varepsilon / 2}^{x}}=0\right)\right)=C_{3.10}(\kappa)|x|^{-p},
$$

and for any $x \in S\left(X_{0}\right)^{c}$,

$$
\begin{aligned}
\lim _{\varepsilon \downarrow 0} \mathbb{E}_{X_{0}}\left(\frac{X_{G_{\varepsilon}^{x}}(1)}{\varepsilon^{p}} \exp \left(-\kappa \frac{X_{G_{\varepsilon}^{x}}(1)}{\varepsilon^{2}}\right) 1\left(X_{G_{\varepsilon / 2}^{x}}=0\right)\right) \\
=e^{-\int V^{\infty}(y-x) X_{0}(d y)} \int C_{3.10}(\kappa)|y-x|^{-p} X_{0}(d y) .
\end{aligned}
$$

Moreover, for any $\kappa>0$ and $x \neq 0$, we have

$$
\mathbb{N}_{0}\left(\frac{X_{G_{\varepsilon}^{x}}(1)}{\varepsilon^{p}} \exp \left(-\kappa \frac{X_{G_{\varepsilon}^{x}}(1)}{\varepsilon^{2}}\right) 1\left(X_{G_{\varepsilon / 2}^{x}}=0\right)\right) \leq|x|^{-p}, \forall 0<\varepsilon<|x| .
$$




\section{Second moment convergence}

One important step in proving the existence of the limiting measure in Theorems 1.3, 1.4 and Theorems $1.13,1.14$ is the exact convergence of the second moment measures, that is to say for any $x_{1} \neq x_{2}$, the limits

$$
\left\{\begin{array}{l}
\lim _{\lambda_{1}, \lambda_{2} \rightarrow \infty} \lambda_{1}^{1+\alpha} \lambda_{2}^{1+\alpha} \mathbb{N}_{x}\left(L^{x_{1}} L^{x_{2}} \exp \left(-\sum_{i=1}^{2} \lambda_{i} L^{x_{i}}\right)\right) \\
\lim _{\varepsilon_{1}, \varepsilon_{2} \downarrow 0} \mathbb{N}_{x}\left(\prod_{i=1}^{2} \frac{X_{G_{i}}^{x_{i}}(1)}{\varepsilon_{i}^{p}} \exp \left(-\kappa \frac{X_{G_{i}}^{x_{i}}(1)}{\varepsilon_{i}^{2}}\right) 1\left(X_{G_{\varepsilon_{i} / 2}^{x_{i}}}=0\right)\right)
\end{array}\right.
$$

exist for all $x \neq x_{1}, x_{2}$. Similarly for any $x_{1}, x_{2} \in S\left(X_{0}\right)^{c}$, the existence of the following limits is required for $\mathbb{P}_{\delta_{0}}$ and $\mathbb{P}_{X_{0}}$ case:

$$
\left\{\begin{array}{l}
\lim _{\lambda_{1}, \lambda_{2} \rightarrow \infty} \lambda_{1}^{1+\alpha} \lambda_{2}^{1+\alpha} \mathbb{E}_{X_{0}}\left(L^{x_{1}} L^{x_{2}} \exp \left(-\sum_{i=1}^{2} \lambda_{i} L^{x_{i}}\right)\right) \\
\lim _{\varepsilon_{1}, \varepsilon_{2} \downarrow 0} \mathbb{E}_{X_{0}}\left(\prod_{i=1}^{2} \frac{X_{G_{\varepsilon_{i}}^{x_{i}}}(1)}{\varepsilon_{i}^{p}} \exp \left(-\kappa \frac{X_{G_{\varepsilon_{i}}^{x_{i}}}(1)}{\varepsilon_{i}^{2}}\right) 1\left(X_{G_{\varepsilon_{i} / 2}^{x_{i}}}=0\right)\right) .
\end{array}\right.
$$

We first introduce some notations. For $x_{1} \neq x_{2}$, we let $\vec{x}=\left(x_{1}, x_{2}\right)$ and $\vec{\lambda}=\left(\lambda_{1}, \lambda_{2}\right) \in$ $[0, \infty)^{2} \backslash\{(0,0)\}$. Define $V^{\vec{\lambda}, \vec{x}} \geq 0$ to be

$$
V^{\vec{\lambda}, \vec{x}}(x) \equiv \mathbb{N}_{x}\left(1-\exp \left(-\sum_{i=1}^{2} \lambda_{i} L^{x_{i}}\right)\right), \forall x \neq x_{1}, x_{2},
$$

so that for any $X_{0} \in M_{F}$ with $d\left(x_{i}, S\left(X_{0}\right)\right)>0, i=1,2$,

$$
\mathbb{E}_{X_{0}}\left(\exp \left(-\sum_{i=1}^{2} \lambda_{i} L^{x_{i}}\right)\right)=\exp \left(-X_{0}\left(V^{\vec{\lambda}, \vec{x}}\right)\right)
$$

where (4.4) follows by (2.2) (see also Lemma 9.1 of [19]). Pick $\varepsilon_{1}, \varepsilon_{2}>0$ small enough so that $B\left(x_{1}, \varepsilon_{1}\right) \cap B\left(x_{2}, \varepsilon_{2}\right)=\emptyset$. Let $\vec{\varepsilon}=\left(\varepsilon_{1}, \varepsilon_{2}\right)$ and $G=G_{\varepsilon_{1}}^{x_{1}} \cap G_{\varepsilon_{2}}^{x_{2}}$. Define $U^{\vec{\lambda}, \vec{x}, \vec{\varepsilon}} \geq 0$ to be

$$
U^{\vec{\lambda}, \vec{x}, \vec{\varepsilon}}(x) \equiv \mathbb{N}_{x}\left(1-\prod_{i=1}^{2} \exp \left(-\lambda_{i} \frac{X_{G_{\varepsilon_{i}}^{x_{i}}}(1)}{\varepsilon_{i}^{2}}\right) 1\left(X_{G_{\varepsilon_{i} / 2}^{x_{i}}}=0\right)\right), \forall x \in G,
$$

so that for any $X_{0} \in M_{F}$ with $d\left(S\left(X_{0}\right), G^{c}\right)>0$,

$$
\mathbb{E}_{X_{0}}\left(\prod_{i=1}^{2} \exp \left(-\lambda_{i} \frac{X_{G_{\varepsilon_{i}}^{x_{i}}}(1)}{\varepsilon_{i}^{2}}\right) 1\left(X_{G_{\varepsilon_{i} / 2}^{x_{i}}}=0\right)\right)=\exp \left(-X_{0}\left(U^{\vec{\lambda}, \vec{x}, \vec{\varepsilon}}\right)\right) .
$$

The proof of (4.6) follows easily from the monotone convergence theorem:

$$
\begin{aligned}
& \mathbb{E}_{X_{0}}\left(\prod_{i=1}^{2} \exp \left(-\lambda_{i} \frac{X_{G_{\varepsilon_{i}}^{x_{i}}}(1)}{\varepsilon_{i}^{2}}\right) 1\left(X_{G_{\varepsilon_{i} / 2}^{x_{i}}}=0\right)\right) \\
= & \lim _{n \rightarrow \infty} \mathbb{E}_{X_{0}}\left(\exp \left(-\sum_{i=1}^{2} \lambda_{i} \frac{X_{G_{\varepsilon_{i}}^{x_{i}}}(1)}{\varepsilon_{i}^{2}}-\sum_{i=1}^{2} n X_{G_{\varepsilon_{i} / 2}^{x_{i}}}(1)\right)\right) \\
= & \lim _{n \rightarrow \infty} \exp \left(-\int \mathbb{N}_{x}\left(1-\exp \left(-\sum_{i=1}^{2} \lambda_{i} \frac{X_{G_{\varepsilon_{i}}^{x_{i}}}(1)}{\varepsilon_{i}^{2}}-\sum_{i=1}^{2} n X_{G_{\varepsilon_{i} / 2}^{x_{i}}}(1)\right)\right) X_{0}(d x)\right) \\
= & \exp \left(-X_{0}\left(U^{\vec{\lambda}, \vec{x}, \vec{\varepsilon}}\right)\right),
\end{aligned}
$$

where the second equality follows from the Poisson decomposition (2.5). 
The monotone convergence theorem and the convexity of $e^{-a x}$ for $a, x>0$ allow us to differentiate (4.5) with respect to $\lambda_{i}>0$ and then further differentiate with respect to $\lambda_{3-i}>0$ to get

$$
\begin{aligned}
& U_{i}^{\vec{\lambda}, \vec{x}, \vec{\varepsilon}}(x):=\frac{d}{d \lambda_{i}} U^{\vec{\lambda}, \vec{x}, \vec{\varepsilon}}(x) \\
& =\mathbb{N}_{x}\left(\frac{X_{G_{\varepsilon_{i}}^{x_{i}}}(1)}{\varepsilon_{i}^{2}} \prod_{j=1}^{2} \exp \left(-\lambda_{j} \frac{X_{G_{\varepsilon_{j}}^{x_{j}}}(1)}{\varepsilon_{j}^{2}}\right) 1\left(X_{G_{\varepsilon_{j} / 2}^{x_{j}}}^{2}=0\right)\right), i=1,2,
\end{aligned}
$$

and

$$
\begin{aligned}
& U_{1,2}^{\vec{\lambda}, \vec{x}, \vec{\varepsilon}}(x):=\frac{d^{2}}{d \lambda_{1} d \lambda_{2}} U^{\vec{\lambda}, \vec{x}, \vec{\varepsilon}}(x) \\
& =-\mathbb{N}_{x}\left(\prod_{i=1}^{2} \frac{X_{G_{\varepsilon_{i}}^{x_{i}}}(1)}{\varepsilon_{i}^{2}} \exp \left(-\lambda_{i} \frac{X_{G_{\varepsilon_{i}}^{x_{i}}(1)}}{\varepsilon_{i}^{2}}\right) 1\left(X_{G_{\varepsilon_{i} / 2}^{x_{i}}}=0\right)\right) .
\end{aligned}
$$

Similarly we can differentiate (4.3) to get

$$
V_{i}^{\vec{\lambda}, \vec{x}}(x):=\frac{d}{d \lambda_{i}} V^{\vec{\lambda}, \vec{x}}(x)=\mathbb{N}_{x}\left(L^{x_{i}} \exp \left(-\sum_{j=1}^{2} \lambda_{j} L^{x_{j}}\right)\right), i=1,2,
$$

and

$$
V_{1,2}^{\vec{\lambda}, \vec{x}}(x):=\frac{d^{2}}{d \lambda_{1} d \lambda_{2}} V^{\vec{\lambda}, \vec{x}}(x)=-\mathbb{N}_{x}\left(L^{x_{1}} L^{x_{2}} \exp \left(-\sum_{i=1}^{2} \lambda_{i} L^{x_{i}}\right)\right) .
$$

For the general initial condition case, we can also differentiate (4.4) and (4.6) to get

$$
\begin{aligned}
& \mathbb{E}_{X_{0}}\left(\prod_{j=1}^{2} \frac{X_{G_{\varepsilon_{j}}^{x_{j}}}(1)}{\varepsilon_{j}^{2}} \exp \left(-\lambda_{j} \frac{X_{G_{\varepsilon_{j}}^{x_{j}}}(1)}{\varepsilon_{j}^{2}}\right) 1\left(X_{G_{\varepsilon_{j} / 2}^{x_{j}}}=0\right)\right) \\
= & \exp \left(-X_{0}\left(U^{\vec{\lambda}, \vec{x}, \vec{\varepsilon}}\right)\right)\left(X_{0}\left(U_{1}^{\vec{\lambda}, \vec{x}, \vec{\varepsilon}}\right) X_{0}\left(U_{2}^{\vec{\lambda}, \vec{x}, \vec{\varepsilon}}\right)-X_{0}\left(U_{1,2}^{\vec{\lambda}, \vec{x}, \vec{\varepsilon}}\right)\right)
\end{aligned}
$$

and

$$
\begin{aligned}
& \mathbb{E}_{X_{0}}\left(L^{x_{1}} L^{x_{2}} \exp \left(-\sum_{i=1}^{2} \lambda_{i} L^{x_{i}}\right)\right) \\
= & \exp \left(-X_{0}\left(V^{\vec{\lambda}, \vec{x}}\right)\right)\left(X_{0}\left(V_{1}^{\vec{\lambda}, \vec{x}}\right) X_{0}\left(V_{2}^{\vec{\lambda}, \vec{x}}\right)-X_{0}\left(V_{1,2}^{\vec{\lambda}, \vec{x}}\right)\right) .
\end{aligned}
$$

Hence one can see that it suffices to consider the convergence of $U_{i}^{\vec{\lambda}, \vec{x}, \vec{\varepsilon}}(x), V_{i}^{\vec{\lambda}, \vec{x}}(x)$, $i=1,2$ and $U_{1,2}^{\vec{\lambda}, \vec{x}, \vec{\varepsilon}}(x), V_{1,2}^{\vec{\lambda}, \vec{x}}(x)$ for the proofs of (4.1) and (4.2).

Proposition 4.1. Fix any $x_{1} \neq x_{2}$.

(i) There exists some constant $K_{4.1}>0$ so that for all $x \neq x_{1}, x_{2}$,

$$
\lim _{\lambda_{1}, \lambda_{2} \rightarrow \infty} \lambda_{i}^{1+\alpha} V_{i}^{\vec{\lambda}, \vec{x}}(x)=K_{4.1} U_{i}^{\vec{\infty}, \vec{x}}(x), \quad i=1,2,
$$

where $U_{i}^{\vec{\infty}, \vec{x}}$ is as in (1.16). Moreover, $K_{4.1}=c_{3.8}$.

(ii) For any $\lambda_{1}, \lambda_{2}>0$, there exist some constants $C_{4.1}\left(\lambda_{1}\right), C_{4.1}\left(\lambda_{2}\right)>0$ such that for all $x \neq x_{1}, x_{2}$, we have

$$
\lim _{\varepsilon_{1}, \varepsilon_{2} \downarrow 0} \frac{1}{\varepsilon_{i}^{p-2}} U_{i}^{\vec{\lambda}, \vec{x}, \vec{\varepsilon}}(x)=C_{4.1}\left(\lambda_{i}\right) U_{i}^{\vec{\infty}, \vec{x}}(x), i=1,2 .
$$

Moreover, the multiplicative constant $c_{1.13}(\kappa)$ in Theorem 1.13 is $C_{4.1}(\kappa) K_{4.1}^{-1}$. 
Proposition 4.2. Fix any $x_{1} \neq x_{2}$. For all $x \neq x_{1}, x_{2}$, we have

$$
\begin{aligned}
& \text { (i) } \lim _{\lambda_{1}, \lambda_{2} \rightarrow \infty} \lambda_{1}^{1+\alpha} \lambda_{2}^{1+\alpha}\left(-V_{1,2}^{\vec{\lambda}, \vec{x}}(x)\right)=K_{4.1}^{2}\left(-U_{1,2}^{\vec{\infty}, \vec{x}}(x)\right) . \\
& \text { (ii) } \lim _{\varepsilon_{1}, \varepsilon_{2} \downarrow 0} \frac{1}{\varepsilon_{1}^{p-2}} \frac{1}{\varepsilon_{2}^{p-2}}\left(-U_{1,2}^{\vec{\lambda}, \vec{x}, \vec{\varepsilon}}(x)\right)=C_{4.1}\left(\lambda_{1}\right) C_{4.1}\left(\lambda_{2}\right)\left(-U_{1,2}^{\vec{\infty}, \vec{x}}(x)\right) .
\end{aligned}
$$

Here $U_{1,2}^{\vec{\infty}, \vec{x}}$ is as in (1.17) and there is some universal constant $c_{4.2}>0$ such that for all $x \neq x_{1}, x_{2}$,

$$
0 \leq-U_{1,2}^{\vec{\infty}, \vec{x}}(x) \leq c_{4.2}\left(\left|x-x_{1}\right|^{-p}+\left|x-x_{2}\right|^{-p}\right)\left|x_{1}-x_{2}\right|^{2-p} .
$$

The proofs of Propositions 4.1 and 4.2 are long and involved and will be deferred to Sections 8 and 9.

In order to prove that $\widetilde{\mathcal{L}}(\kappa)=c_{1.13}(\kappa) \mathcal{L}$ a.s., we implement ideas from the above: For any $x_{1} \neq x_{2}, \lambda_{1}, \lambda_{2}>0$ and $0<\varepsilon<\left|x_{2}-x_{1}\right| / 4$, we define $W^{\vec{\lambda}, \vec{x}, \varepsilon} \geq 0$ for all $x \neq x_{1}$ and $\left|x-x_{2}\right|>\varepsilon$ by

$$
W^{\vec{\lambda}, \vec{x}, \varepsilon}(x) \equiv \mathbb{N}_{x}\left(1-e^{-\lambda_{1} L^{x_{1}}} \exp \left(-\lambda_{2} \frac{X_{G_{\varepsilon}^{x_{2}}}(1)}{\varepsilon^{2}}\right) 1\left(X_{G_{\varepsilon / 2}^{x_{2}}}=0\right)\right),
$$

so that for any $X_{0} \in M_{F}$ with $d\left(x_{1}, S\left(X_{0}\right)\right)>0$ and $\overline{B\left(x_{2}, \varepsilon\right)} \subset S\left(X_{0}\right)^{c}$,

$$
\mathbb{E}_{X_{0}}\left(\exp \left(-\lambda_{1} L^{x_{1}}-\lambda_{2} \frac{X_{G_{\varepsilon}^{x_{2}}}(1)}{\varepsilon^{2}}\right) 1\left(X_{G_{\varepsilon / 2}^{x_{2}}}=0\right)\right)=\exp \left(-X_{0}\left(W^{\vec{\lambda}, \vec{x}, \varepsilon}\right)\right),
$$

where (4.15) follows as in (4.6). Similar to (4.7) and (4.8), we can differentiate (4.14) with respect to $\lambda_{i}>0$ and then further differentiate with respect to $\lambda_{3-i}>0$ to get

$$
\left\{\begin{aligned}
W_{1}^{\vec{\lambda}, \vec{x}, \varepsilon}(x) & :=\frac{d}{d \lambda_{1}} W^{\vec{\lambda}, \vec{x}, \varepsilon}(x) \\
& =\mathbb{N}_{x}\left(L^{x_{1}} e^{-\lambda_{1} L^{x_{1}}} \exp \left(-\lambda_{2} \frac{X_{G_{\varepsilon}^{x_{2}}}(1)}{\varepsilon^{2}}\right) 1\left(X_{G_{\varepsilon / 2}^{x_{2}}}=0\right)\right) \\
W_{2}^{\vec{\lambda}, \vec{x}, \varepsilon}(x) & :=\frac{d}{d \lambda_{2}} W^{\vec{\lambda}, \vec{x}, \varepsilon}(x) \\
& =\mathbb{N}_{x}\left(\frac{X_{G_{\varepsilon}}^{x_{2}}(1)}{\varepsilon^{2}} \exp \left(-\lambda_{2} \frac{X_{G_{\varepsilon} x_{2}(1)}}{\varepsilon^{2}}\right) 1\left(X_{G_{\varepsilon / 2}^{x_{2}}}=0\right) e^{-\lambda_{1} L^{x_{1}}}\right)
\end{aligned}\right.
$$

and

$$
\begin{aligned}
& W_{1,2}^{\vec{\lambda}, \vec{x}, \varepsilon}(x):=\frac{d^{2}}{d \lambda_{1} d \lambda_{2}} W^{\vec{\lambda}, \vec{x}, \varepsilon}(x) \\
= & -\mathbb{N}_{x}\left(L^{x_{1}} e^{-\lambda_{1} L^{x_{1}}} \frac{X_{G_{\varepsilon}^{x_{2}}}(1)}{\varepsilon^{2}} \exp \left(-\lambda_{2} \frac{X_{G_{\varepsilon}^{x_{2}}}(1)}{\varepsilon^{2}}\right) 1\left(X_{G_{\varepsilon / 2}^{x_{2}}}=0\right)\right) .
\end{aligned}
$$

For the general initial condition case, we can differentiate (4.15) to get

$$
\begin{aligned}
& \mathbb{E}_{X_{0}}\left(L^{x_{1}} e^{-\lambda_{1} L^{x_{1}}} \frac{X_{G_{\varepsilon}^{x_{2}}}(1)}{\varepsilon^{2}} \exp \left(-\lambda_{2} \frac{X_{G_{\varepsilon}^{x_{2}}}(1)}{\varepsilon^{2}}\right) 1\left(X_{G_{\varepsilon / 2}^{x_{2}}}=0\right)\right) \\
& =\exp \left(-X_{0}\left(W^{\vec{\lambda}, \vec{x}, \varepsilon}\right)\right)\left(X_{0}\left(W_{1}^{\vec{\lambda}, \vec{x}, \varepsilon}\right) X_{0}\left(W_{2}^{\vec{\lambda}, \vec{x}, \varepsilon}\right)-X_{0}\left(W_{1,2}^{\vec{\lambda}, \vec{x}, \varepsilon}\right)\right) .
\end{aligned}
$$

We will also need the following mixture of Propositions 4.1 and 4.2.

Proposition 4.3. Fix any $x_{1} \neq x_{2}$. For all $x \neq x_{1}, x_{2}$, we have

$$
\begin{aligned}
& \text { (i) }\left\{\begin{array}{l}
\lim _{\lambda_{1} \rightarrow \infty, \varepsilon \downarrow 0} \lambda_{1}^{1+\alpha} W_{1}^{\vec{\lambda}, \vec{x}, \varepsilon}(x)=K_{4.1} U_{1}^{\vec{\infty}, \vec{x}}(x), \\
\lim _{\lambda \rightarrow \infty, \varepsilon \downarrow 0} \frac{1}{\varepsilon^{p-2}} W_{2}^{\vec{\lambda}, \vec{x}, \varepsilon}(x)=C_{4.1}\left(\lambda_{2}\right) U_{2}^{\vec{\infty}, \vec{x}}(x) .
\end{array}\right. \\
& \text { (ii) } \lim _{\lambda_{1} \rightarrow \infty, \varepsilon \downarrow 0} \lambda_{1}^{1+\alpha} \frac{1}{\varepsilon^{p-2}}\left(-W_{1,2}^{\vec{\lambda}, \vec{x}, \varepsilon}(x)\right)=K_{4.1} C_{4.1}\left(\lambda_{2}\right)\left(-U_{1,2}^{\vec{\infty}, \vec{x}}(x)\right) .
\end{aligned}
$$

The proof of Proposition 4.3 follows in a similar way to the proofs of Proposition 4.1 and Proposition 4.2 and is deferred to Section 8 and Section 9. We will first proceed to the proof of our main results. 


\section{Proofs of Theorems 1.3 and 1.13 and Theorems 1.8 and 1.10}

In this section, we will finish the proofs of Theorems 1.3, 1.13 and Theorems 1.8, 1.10 assuming Propositions 4.1, 4.2 and 4.3.

\subsection{Preliminaries}

Recall from last section the definitions of $V^{\vec{\lambda}, \vec{x}}, U^{\vec{\lambda}, \vec{x}, \vec{\varepsilon}}$ and $W^{\vec{\lambda}, \vec{x}, \varepsilon}$ and their first and second derivatives and recall $V^{\vec{\infty}, \vec{x}}$ from (1.15). Fix $x_{1} \neq x_{2}$. It is not hard to check that (see Lemma 8.1) for all $x \neq x_{1}, x_{2}$,

$$
\lim _{\varepsilon_{1}, \varepsilon_{2} \downarrow 0} U^{\vec{\lambda}, \vec{x}, \vec{\varepsilon}}(x)=\lim _{\lambda_{1}, \lambda_{2} \rightarrow \infty} V^{\vec{\lambda}, \vec{x}}(x)=\lim _{\lambda_{1} \rightarrow \infty, \varepsilon \downarrow 0} W^{\vec{\lambda}, \vec{x}, \varepsilon}(x)=V^{\vec{\infty}, \vec{x}}(x) .
$$

Recall (4.9) and use Proposition 3.2 to get for all $\lambda_{1}, \lambda_{2}>0$ and $x \neq x_{1}, x_{2}$,

$$
\lambda_{i}^{1+\alpha} V_{i}^{\vec{\lambda}, \vec{x}}(x) \leq \mathbb{N}_{x}\left(\lambda_{i}^{1+\alpha} L^{x_{i}} \exp \left(-\lambda_{i} L^{x_{i}}\right)\right) \leq c_{3.2}\left|x-x_{i}\right|^{-p}, i=1,2 .
$$

Recall (4.7). Similarly we can get for all $\varepsilon_{1}, \varepsilon_{2}, \lambda_{1}, \lambda_{2}>0$ and for all $x$ so that $\left|x-x_{i}\right|>\varepsilon_{i}$, for $i=1,2$,

$$
\frac{1}{\varepsilon_{i}^{p-2}} U_{i}^{\vec{\lambda}, \vec{x}, \vec{\varepsilon}}(x) \leq \mathbb{N}_{x}\left(\frac{X_{G_{\varepsilon_{i}}^{x_{i}}}(1)}{\varepsilon_{i}^{p}} \exp \left(-\lambda_{i} \frac{X_{G_{\varepsilon_{i}}^{x_{i}}}(1)}{\varepsilon_{i}^{2}}\right) 1\left(X_{G_{\varepsilon_{i} / 2}^{x_{i}}}=0\right)\right) \leq\left|x-x_{i}\right|^{-p},
$$

where the last equality is by (3.21). Recall $W_{i}^{\vec{\lambda}, \vec{x}, \varepsilon}(x), i=1,2$ from (4.16). It follows that for any $\lambda_{1}, \lambda_{2}, \varepsilon>0$ and for all $x$ with $x \neq x_{1}$ and $\left|x-x_{2}\right|>\varepsilon$, we have

$$
\lambda_{1}^{1+\alpha} W_{1}^{\vec{\lambda}, \vec{x}, \varepsilon}(x) \leq \lambda_{1}^{1+\alpha} \mathbb{N}_{x}\left(L^{x_{1}} \exp \left(-\lambda_{1} L^{x_{1}}\right)\right) \leq c_{3.2}\left|x-x_{1}\right|^{-p},
$$

and

$$
\frac{1}{\varepsilon^{p-2}} W_{2}^{\vec{\lambda}, \vec{x}, \varepsilon}(x) \leq \mathbb{N}_{x}\left(\frac{X_{G_{\varepsilon}^{x_{2}}}(1)}{\varepsilon^{p}} \exp \left(-\lambda_{2} \frac{X_{G_{\varepsilon}^{x_{2}}}(1)}{\varepsilon^{2}}\right) 1\left(X_{G_{\varepsilon / 2}^{x_{2}}}=0\right)\right) \leq\left|x-x_{2}\right|^{-p} .
$$

The proof of Proposition 6.1 of [19] readily implies that (note $U^{\vec{\lambda}, \vec{x}}$ is used there to denote our $V_{1,2}^{\vec{\lambda}, \vec{x}}$ here) for all $x_{1} \neq x_{2}$, if $\left|x-x_{1}\right| \wedge\left|x-x_{2}\right|>\varepsilon_{0}$ for some $\varepsilon_{0}>0$, then there is some constant $C\left(\varepsilon_{0}\right)>0$ so that

$$
0 \leq \lambda_{1}^{1+\alpha} \lambda_{2}^{1+\alpha}\left(-V_{1,2}^{\vec{\lambda}, \vec{x}}(x)\right) \leq C\left(\varepsilon_{0}\right)\left(1+\left|x_{1}-x_{2}\right|^{2-p}\right), \quad \forall \lambda_{1}, \lambda_{2} \geq 1 .
$$

Similarly one can show that (see Lemma 9.5) for all $\varepsilon_{1}, \varepsilon_{2}>0$ small,

$$
0 \leq \frac{1}{\varepsilon_{1}^{p-2}} \frac{1}{\varepsilon_{2}^{p-2}}\left(-U_{1,2}^{\vec{\lambda}, \vec{x}, \vec{\varepsilon}}(x)\right) \leq C\left(\varepsilon_{0}\right)\left(1+\left|x_{1}-x_{2}\right|^{2-p}\right),
$$

and for all $\lambda_{1} \geq 1$ large and $\varepsilon>0$ small,

$$
\left.0 \leq \lambda_{1}^{1+\alpha} \frac{1}{\varepsilon^{p-2}}\left(-W_{1,2}^{\vec{\lambda}, \vec{x}, \varepsilon}(x)\right)\right) \leq C\left(\varepsilon_{0}\right)\left(1+\left|x_{1}-x_{2}\right|^{2-p}\right) .
$$

Theorem 5.1. For any bounded Borel function $h: \mathbb{R}^{d} \times \mathbb{R}^{d} \rightarrow \mathbb{R}$ supported on $\left\{\left(x_{1}, x_{2}\right)\right.$ : $\left.\varepsilon_{0} \leq\left|x_{1}\right|,\left|x_{2}\right| \leq \varepsilon_{0}^{-1}\right\}$ for some $\varepsilon_{0}>0$, we have
(a) $\lim _{\lambda_{1}, \lambda_{2} \rightarrow \infty} \mathbb{N}_{0}\left(\left(\mathcal{L}^{\lambda_{1}} \times \mathcal{L}^{\lambda_{2}}\right)(h)\right)=K_{4.1}^{2} \int h\left(x_{1}, x_{2}\right)\left(-U_{1,2}^{\vec{\infty}, \vec{x}}(0)\right) d x_{1} d x_{2}$,
(b) $\lim _{\varepsilon_{1}, \varepsilon_{2} \downarrow 0} \mathbb{N}_{0}\left(\left(\widetilde{\mathcal{L}}(\kappa)^{\varepsilon_{1}} \times \widetilde{\mathcal{L}}(\kappa)^{\varepsilon_{2}}\right)(h)\right)=C_{4.1}(\kappa)^{2} \int h\left(x_{1}, x_{2}\right)\left(-U_{1,2}^{\vec{\infty}, \vec{x}}(0)\right) d x_{1} d x_{2}$. 
Proof. It suffices to consider nonnegative bounded Borel function $h$. By an application of Fubini's theorem, we have

$$
\begin{aligned}
& \mathbb{N}_{0}\left(\left(\mathcal{L}^{\lambda_{1}} \times \mathcal{L}^{\lambda_{2}}\right)(h)\right) \\
= & \int_{\varepsilon_{0} \leq\left|x_{1}\right|,\left|x_{2}\right| \leq \varepsilon_{0}^{-1}} h\left(x_{1}, x_{2}\right) \mathbb{N}_{0}\left(\lambda_{1}^{1+\alpha} \lambda_{2}^{1+\alpha} L^{x_{1}} L^{x_{2}} e^{-\lambda_{1} L^{x_{1}}} e^{-\lambda_{2} L^{x_{2}}}\right) d x_{1} d x_{2} \\
= & \int_{\varepsilon_{0} \leq\left|x_{1}\right|,\left|x_{2}\right| \leq \varepsilon_{0}^{-1}} h\left(x_{1}, x_{2}\right) \lambda_{1}^{1+\alpha} \lambda_{2}^{1+\alpha}\left(-V_{1,2}^{\vec{\lambda}, \vec{x}}(0)\right) d x_{1} d x_{2},
\end{aligned}
$$

where the second equality is by (4.10). Since $h$ is bounded, in view of (5.6) we can see that the integrand has an integrable bound and so (a) will follow immediately by the dominated convergence theorem using Proposition 4.2(i). Similarly (b) will follow from Proposition 4.2(ii) and (5.7).

Corollary 5.2. For any bounded Borel function $\phi$ on $\mathbb{R}^{d}$ and for any $k \geq 1$, we have $\mathcal{L}^{\lambda}\left(\phi \cdot 1\left(k^{-1} \leq|\cdot| \leq k\right)\right)$ converges in $L^{2}\left(\mathbb{N}_{0}\right)$ as $\lambda \rightarrow \infty$ and $\widetilde{\mathcal{L}}(\kappa)^{\varepsilon}\left(\phi \cdot 1\left(k^{-1} \leq|\cdot| \leq k\right)\right)$ converges in $L^{2}\left(\mathbb{N}_{0}\right)$ as $\varepsilon \downarrow 0$.

Proof. For any bounded Borel function $\phi$ we let

$$
h\left(x_{1}, x_{2}\right)=\phi\left(x_{1}\right) 1\left(k^{-1} \leq\left|x_{1}\right| \leq k\right) \cdot \phi\left(x_{2}\right) 1\left(k^{-1} \leq\left|x_{2}\right| \leq k\right),
$$

and apply Theorem 5.1(b) with the above $h$ to get

$$
\begin{aligned}
& \quad \lim _{\varepsilon_{1}, \varepsilon_{2} \downarrow 0} \mathbb{N}_{0}\left(\left(\widetilde{\mathcal{L}}(\kappa)^{\varepsilon_{1}}\left(\phi \cdot 1\left(k^{-1} \leq|\cdot| \leq k\right)\right)-\widetilde{\mathcal{L}}(\kappa)^{\varepsilon_{2}}\left(\phi \cdot 1\left(k^{-1} \leq|\cdot| \leq k\right)\right)\right)^{2}\right) \\
& =\lim _{\varepsilon_{1}, \varepsilon_{2} \downarrow 0} \mathbb{N}_{0}\left(\left(\widetilde{\mathcal{L}}(\kappa)^{\varepsilon_{1}} \times \widetilde{\mathcal{L}}(\kappa)^{\varepsilon_{1}}\right)(h)\right)-2 \mathbb{N}_{0}\left(\left(\widetilde{\mathcal{L}}(\kappa)^{\varepsilon_{1}} \times \widetilde{\mathcal{L}}(\kappa)^{\varepsilon_{2}}\right)(h)\right) \\
& \quad+\mathbb{N}_{0}\left(\left(\widetilde{\mathcal{L}}(\kappa)^{\varepsilon_{2}} \times \widetilde{\mathcal{L}}(\kappa)^{\varepsilon_{2}}\right)(h)\right)=0 .
\end{aligned}
$$

Therefore $\left\{\widetilde{\mathcal{L}}(\kappa)^{\varepsilon}\left(\phi \cdot 1\left(k^{-1} \leq|\cdot| \leq k\right)\right): \varepsilon>0\right\}$ is a Cauchy sequence in $L^{2}\left(\mathbb{N}_{0}\right)$ as $\varepsilon \downarrow 0$ and so converges in $L^{2}\left(\mathbb{N}_{0}\right)$. The case for $\mathcal{L}^{\lambda}$ is similar.

Corollary 5.3. For any bounded Borel function $\phi$ on $\mathbb{R}^{d}$ and for any $k \geq 1$, we have $C_{4.1}(\kappa) \mathcal{L}^{\lambda}\left(\phi \cdot 1_{\left(k^{-1} \leq|\cdot| \leq k\right)}\right)-K_{4.1} \widetilde{\mathcal{L}}(\kappa)^{\varepsilon}\left(\phi \cdot 1_{\left(k^{-1} \leq|\cdot| \leq k\right)}\right)$ converges to 0 in $L^{2}\left(\overline{\mathbb{N}}_{0}\right)$ as $\lambda \rightarrow \infty$ and $\varepsilon \downarrow 0$.

Proof. It suffices to prove for nonnegative $\phi \geq 0$. Let $h\left(x_{1}, x_{2}\right)$ be as in (5.9) and use Fubini's theorem to get

$$
\begin{aligned}
& \mathbb{N}_{0}\left(\mathcal{L}^{\lambda}\left(\phi \cdot 1_{\left(k^{-1} \leq|\cdot| \leq k\right)}\right) \times \widetilde{\mathcal{L}}(\kappa)^{\varepsilon}\left(\phi \cdot 1_{\left(k^{-1} \leq|\cdot| \leq k\right)}\right)\right) \\
= & \int h\left(x_{1}, x_{2}\right) \mathbb{N}_{0}\left(\lambda^{1+\alpha} L^{x_{1}} e^{-\lambda L^{x_{1}}} \frac{X_{G_{\varepsilon}^{x_{2}}(1)}}{\varepsilon^{p}} e^{-\kappa \frac{x_{G_{\varepsilon}^{x_{2}(1)}}^{\varepsilon^{2}}}{{ }^{2}}} 1_{\left\{X_{G_{\varepsilon / 2} x_{2}}=0\right\}}\right) d x_{1} d x_{2} \\
= & \int_{k^{-1} \leq\left|x_{1}\right|,\left|x_{2}\right| \leq k, x_{1} \neq x_{2}} h\left(x_{1}, x_{2}\right) \lambda^{1+\alpha} \frac{1}{\varepsilon^{p-2}}\left(-W_{1,2}^{\vec{\lambda}, \vec{x}, \varepsilon}(0)\right) d x_{1} d x_{2},
\end{aligned}
$$

where $\vec{\lambda}=(\lambda, \kappa)$. Now apply Proposition 4.3(ii), (5.8) and the dominated convergence theorem to conclude

$$
\begin{aligned}
\lim _{\lambda \rightarrow \infty, \varepsilon \downarrow 0} & \mathbb{N}_{0}\left(\mathcal{L}^{\lambda}\left(\phi \cdot 1_{\left(k^{-1} \leq|\cdot| \leq k\right)}\right) \times \widetilde{\mathcal{L}}(\kappa)^{\varepsilon}\left(\phi \cdot 1_{\left(k^{-1} \leq|\cdot| \leq k\right)}\right)\right) \\
& =K_{4.1} C_{4.1}(\kappa) \int_{k^{-1} \leq\left|x_{1}\right|,\left|x_{2}\right| \leq k, x_{1} \neq x_{2}} h\left(x_{1}, x_{2}\right)\left(-U_{1,2}^{\vec{\infty}, \vec{x}}(0)\right) d x_{1} d x_{2} .
\end{aligned}
$$


Therefore

$$
\begin{aligned}
& \lim _{\lambda \rightarrow \infty, \varepsilon \downarrow 0} \mathbb{N}_{0}\left(\left(C_{4.1}(\kappa) \mathcal{L}^{\lambda}\left(\phi \cdot 1_{\left(k^{-1} \leq|\cdot| \leq k\right)}\right)-K_{4.1} \widetilde{\mathcal{L}}(\kappa)^{\varepsilon}\left(\phi \cdot 1_{\left(k^{-1} \leq|\cdot| \leq k\right)}\right)\right)^{2}\right) \\
= & \lim _{\lambda \rightarrow \infty, \varepsilon \downarrow 0} C_{4.1}(\kappa)^{2} \mathbb{N}_{0}\left(\left(\mathcal{L}^{\lambda} \times \mathcal{L}^{\lambda}\right)(h)\right)+K_{4.1}^{2} \mathbb{N}_{0}\left(\left(\widetilde{\mathcal{L}}(\kappa)^{\varepsilon} \times \widetilde{\mathcal{L}}(\kappa)^{\varepsilon}\right)(h)\right) \\
& -2 K_{4.1} C_{4.1}(\kappa) \mathbb{N}_{0}\left(\mathcal{L}^{\lambda}\left(\phi \cdot 1_{\left(k^{-1} \leq|\cdot| \leq k\right)}\right) \times \widetilde{\mathcal{L}}^{\varepsilon}\left(\phi \cdot 1_{\left(k^{-1} \leq|\cdot| \leq k\right)}\right)\right)=0,
\end{aligned}
$$

where we have used Theorem 5.1 and (5.10) in the last equality.

We continue to accommodate $\mathbb{P}_{X_{0}}$ for the general initial condition case.

Theorem 5.4. For any bounded Borel function $h: \mathbb{R}^{d} \times \mathbb{R}^{d} \rightarrow \mathbb{R}$ supported on the set $\left\{\left(x_{1}, x_{2}\right): x_{i} \in S\left(X_{0}\right)^{>\varepsilon_{0}} \cap B\left(\varepsilon_{0}^{-1}\right), i=1,2\right\}$ for some $\varepsilon_{0}>0$, we have

$$
\left\{\begin{array}{c}
\lim _{\lambda_{1}, \lambda_{2} \rightarrow \infty} \mathbb{E}_{X_{0}}\left(\left(\mathcal{L}^{\lambda_{1}} \times \mathcal{L}^{\lambda_{2}}\right)(h)\right)=K_{4.1}^{2} \int h\left(x_{1}, x_{2}\right) \\
e^{-X_{0}\left(V^{\vec{\infty}, \vec{x}}\right)}\left(X_{0}\left(U_{1}^{\vec{\infty}, \vec{x}}\right) X_{0}\left(U_{2}^{\vec{\infty}, \vec{x}}\right)-X_{0}\left(U_{1,2}^{\vec{\infty}, \vec{x}}\right)\right) d x_{1} d x_{2}, \\
\lim _{\varepsilon_{1}, \varepsilon_{2} \downarrow 0} \mathbb{E}_{X_{0}}\left(\left(\widetilde{\mathcal{L}}(\kappa)^{\varepsilon_{1}} \times \widetilde{\mathcal{L}}(\kappa)^{\varepsilon_{2}}\right)(h)\right)=C_{4.1}(\kappa)^{2} \int h\left(x_{1}, x_{2}\right) \\
e^{-X_{0}\left(V^{\vec{\infty}, \vec{x}}\right)}\left(X_{0}\left(U_{1}^{\vec{\infty}, \vec{x}}\right) X_{0}\left(U_{2}^{\vec{\infty}, \vec{x}}\right)-X_{0}\left(U_{1,2}^{\vec{\infty}, \vec{x}}\right)\right) d x_{1} d x_{2} .
\end{array}\right.
$$

Proof. It suffices to prove for nonnegative $h$. By Fubini's theorem and (4.12), we have

$$
\begin{aligned}
& \mathbb{E}_{X_{0}}\left(\left(\mathcal{L}^{\lambda_{1}} \times \mathcal{L}^{\lambda_{2}}\right)(h)\right)=\int_{x_{1}, x_{2} \in B\left(\varepsilon_{0}^{-1}\right) \cap S\left(X_{0}\right)>\varepsilon_{0}} h\left(x_{1}, x_{2}\right) \\
& e^{-X_{0}\left(V^{\vec{\lambda}, \vec{x}}\right)}\left(\lambda_{1}^{1+\alpha} \lambda_{2}^{1+\alpha} X_{0}\left(V_{1}^{\vec{\lambda}, \vec{x}}\right) X_{0}\left(V_{2}^{\vec{\lambda}, \vec{x}}\right)-\lambda_{1}^{1+\alpha} \lambda_{2}^{1+\alpha} X_{0}\left(V_{1,2}^{\vec{\lambda}, \vec{x}}\right)\right) d x_{1} d x_{2} .
\end{aligned}
$$

The result follows by an application of the dominated convergence theorem using Proposition 4.1(i), Proposition 4.2(i), (5.1), (5.2), (5.6), and the assumption on $h$. The case for $\widetilde{\mathcal{L}}(\kappa)^{\varepsilon}$ follows in a similar way.

Corollary 5.5. For any bounded Borel function $\phi$ on $\mathbb{R}^{d}$ and for any $k \geq 1$, we have $\mathcal{L}^{\lambda}\left(\phi \cdot 1_{\left\{B_{k} \cap S\left(X_{0}\right)^{>1 / k}\right\}}\right)$ converges in $L^{2}\left(\mathbb{P}_{X_{0}}\right)$ as $\lambda \rightarrow \infty$ and $\widetilde{\mathcal{L}}(\kappa)^{\varepsilon}\left(\phi \cdot 1_{\left\{B_{k} \cap S\left(X_{0}\right)>1 / k\right\}}\right)$ converges in $L^{2}\left(\mathbb{P}_{X_{0}}\right)$ as $\varepsilon \downarrow 0$.

Proof. For any bounded Borel function $\phi$ we let

$$
h\left(x_{1}, x_{2}\right)=\phi\left(x_{1}\right) 1_{\left\{B_{k} \cap S\left(X_{0}\right)>1 / k\right\}}\left(x_{1}\right) \cdot \phi\left(x_{2}\right) 1_{\left\{B_{k} \cap S\left(X_{0}\right)>1 / k\right\}}\left(x_{2}\right) .
$$

Then the proof follows in a similar way to that of Corollary 5.2 by applying Theorem 5.4 with the above $h$.

Corollary 5.6. For any bounded Borel function $\phi$ on $\mathbb{R}^{d}$ and for any $k \geq 1$, we have $C_{4.1}(\kappa) \mathcal{L}^{\lambda}\left(\phi \cdot 1_{\left\{B_{k} \cap S\left(X_{0}\right)^{>1 / k\}}\right.}\right)-K_{4.1} \widetilde{\mathcal{L}}(\kappa)^{\varepsilon}\left(\phi \cdot 1_{\left\{B_{k} \cap S\left(X_{0}\right)>1 / k\right\}}\right)$ converges to 0 in $L^{2}\left(\mathbb{P}_{X_{0}}\right)$ as $\lambda \rightarrow \infty$ and $\varepsilon \downarrow 0$.

Proof. The proof is similar to that of Corollary 5.3 by using (4.18), (5.1), (5.4), (5.5), (5.8), Proposition 4.3 and Theorem 5.4. 


\subsection{Proofs of Theorems 1.3 and 1.13}

Proposition 5.7. For any $k \geq 1$ and any sequence $\varepsilon_{n} \downarrow 0$, we have $\mathbb{N}_{0}$-a.e. and $\mathbb{P}_{X_{0}}$-a.s. that $\widetilde{\mathcal{L}}(\kappa)^{\varepsilon_{n}}(\mathcal{R})=0$ for all $\varepsilon_{n}>0$ and $\widetilde{\mathcal{L}}(\kappa)^{\varepsilon_{n}}\left(\mathcal{R}^{>1 / k}\right)=0$ for all $0<\varepsilon_{n}<1 / k$.

Proof. First for any $\varepsilon>0$,

$$
\begin{aligned}
& \mathbb{N}_{0}\left(\widetilde{\mathcal{L}}(\kappa)^{\varepsilon}(\mathcal{R})\right) \leq \mathbb{N}_{0}\left(\int \frac{X_{G_{\varepsilon}^{x}}(1)}{\varepsilon^{p}} \exp \left(-\kappa \frac{X_{G_{\varepsilon}^{x}}(1)}{\varepsilon^{2}}\right) 1_{\left(X_{G_{\varepsilon / 2}^{x}}=0\right)} 1_{(x \in \mathcal{R})} d x\right) \\
= & \int \mathbb{N}_{0}\left(\frac{X_{G_{\varepsilon}^{x}}(1)}{\varepsilon^{p}} \exp \left(-\kappa \frac{X_{G_{\varepsilon}^{x}}(1)}{\varepsilon^{2}}\right) 1_{\left(X_{G_{\varepsilon / 2}^{x}}=0\right)} 1_{(x \in \mathcal{R})}\right) d x \\
= & \int \mathbb{N}_{0}\left(\frac{X_{G_{\varepsilon}^{x}}(1)}{\varepsilon^{p}} \exp \left(-\kappa \frac{X_{G_{\varepsilon}^{x}}(1)}{\varepsilon^{2}}\right) 1_{\left(X_{G_{\varepsilon / 2}^{x}}=0\right)} \mathbb{P}_{X_{G_{\varepsilon / 2}^{x}}}(x \in \mathcal{R})\right) d x=0,
\end{aligned}
$$

where the first equality is by Fubini's theorem and the second equality uses Proposition 2.1(ii). Hence $\widetilde{\mathcal{L}}(\kappa)^{\varepsilon}(\mathcal{R})=0, \mathbb{N}_{0}$-a.e.

Next for all $x \in \mathcal{R}^{>1 / k}$ and $0<\varepsilon<1 / k$, we have $\overline{B_{\varepsilon}(x)} \subset \mathcal{R}^{c}$ and (2.4) will then imply $X_{G_{\varepsilon}^{x}}(1)=0$. Thus if $0<\varepsilon<1 / k$,

$$
\begin{aligned}
& \mathbb{N}_{0}\left(\widetilde{\mathcal{L}}(\kappa)^{\varepsilon}\left(\mathcal{R}^{>1 / k}\right)\right) \leq \mathbb{N}_{0}\left(\int \frac{X_{G_{\varepsilon}^{x}}(1)}{\varepsilon^{p}} \exp \left(-\kappa \frac{X_{G_{\varepsilon}^{x}}(1)}{\varepsilon^{2}}\right) 1_{\overline{B_{\varepsilon}(x)} \subset \mathcal{R}^{c}} d x\right) \\
& =\int \mathbb{N}_{0}\left(\frac{X_{G_{\varepsilon}^{x}}(1)}{\varepsilon^{p}} \exp \left(-\kappa \frac{X_{G_{\varepsilon}^{x}}(1)}{\varepsilon^{2}}\right) 1 \frac{}{B_{\varepsilon}(x)} \subset \mathcal{R}^{c}\right) d x=0 .
\end{aligned}
$$

Take a countable union of null sets to see that $\mathbb{N}_{0}$-a.e. $\widetilde{\mathcal{L}}(\kappa)^{\varepsilon_{n}}(\mathcal{R})=0$ for all $\varepsilon_{n}>0$ and $\widetilde{\mathcal{L}}(\kappa)^{\varepsilon_{n}}\left(\mathcal{R}^{>1 / k}\right)=0$ for all $0<\varepsilon_{n}<1 / k$ and so the proof for $\mathbb{N}_{0}$ is complete. The proof for $\mathbb{P}_{X_{0}}$ follows in a similar way.

Proof of Theorems 1.3 and 1.13. We first give the convergence of $\mathcal{L}^{\lambda}$ to $\mathcal{L}$ and $\widetilde{\mathcal{L}}(\kappa)^{\varepsilon}$ to $\widetilde{\mathcal{L}}(\kappa)$ and then find some constant $c_{1.13}(\kappa)>0$ so that $\widetilde{\mathcal{L}}(\kappa)=c_{1.13}(\kappa) \mathcal{L}$ a.s. Next we show that the support of $\widetilde{\mathcal{L}}(\kappa)$ is contained in $\partial \mathcal{R}$ and it follows that the support of $\mathcal{L}$ will also be on $\partial \mathcal{R}$, thus finishing both proofs of Theorem 1.3 and Theorem 1.13. Since the proof for the convergence of $\mathcal{L}^{\lambda}$ and $\widetilde{\mathcal{L}}(\kappa)^{\varepsilon}$ are similar, we will only give the proof for the latter.

We first deal with $\mathbb{N}_{0}$. Let $\left\{\phi_{m}\right\}_{m=1}^{\infty}$ be a countable determining class for $M_{F}\left(\mathbb{R}^{d}\right)$ consisting of bounded, continuous functions and we take $\phi_{1}=1$. Consider

$$
\mathcal{C}=\left\{\psi_{m, k}: \psi_{m, k}=\phi_{m} \chi_{k}, m \geq 1, k \geq 1\right\},
$$

where $\chi_{k}$ is defined by

$$
\chi_{k}(x)= \begin{cases}1, & \text { if } k^{-1} \leq|x| \leq k \\ 0, & \text { if }|x| \leq(2 k)^{-1} \text { or }|x| \geq k+1 \\ \text { continuous, } & \text { on }(2 k)^{-1} \leq|x| \leq k^{-1} \text { and } k \leq|x| \leq k+1\end{cases}
$$

Corollary 5.2 implies that for any $\psi_{m, k} \in \mathcal{C}$, we have $\widetilde{\mathcal{L}}(\kappa)^{\varepsilon}\left(\psi_{m, k}\right)$ converges in $L^{2}\left(\mathbb{N}_{0}\right)$ to some $\widetilde{l}\left(\psi_{m, k}\right)$ in $L^{2}\left(\mathbb{N}_{0}\right)$ and by taking a subsequence we get almost sure convergence. Define subsequences iteratively and take a diagonal subsequence $\varepsilon_{n} \downarrow 0$ (we may assume for all $n \geq 1$ that $0<\varepsilon_{n}<1$ ) to get

$$
\widetilde{\mathcal{L}}(\kappa)^{\varepsilon_{n}}\left(\psi_{m, k}\right) \rightarrow \widetilde{l}\left(\psi_{m, k}\right) \text { as } \varepsilon_{n} \downarrow 0, \text { for all } m, k \geq 1, \mathbb{N}_{0} \text {-a.e. }
$$

Fix $\omega$ outside a null set such that (5.13) hold. Choose $m=1$ in (5.13) to see that $\widetilde{\mathcal{L}}(\kappa)^{\varepsilon_{n}}\left(\chi_{k}\right) \rightarrow \widetilde{l}\left(\chi_{k}\right)$ for all $k \geq 1$. Note we have $\widetilde{l}\left(\chi_{k}\right)<\infty$ by the choice of $\omega$ and so $\mathbb{N}_{0}$-a.e. we have

$$
\sup _{\varepsilon_{n}>0} \widetilde{\mathcal{L}}(\kappa)^{\varepsilon_{n}}\left(\left\{x: k^{-1} \leq|x| \leq k\right\}\right) \leq \sup _{\varepsilon_{n}>0} \widetilde{\mathcal{L}}(\kappa)^{\varepsilon_{n}}\left(\chi_{k}\right)<\infty, \forall k \geq 1 .
$$


The proof of Theorem 1.5 in [9] implies that $\mathbb{N}_{0}$-a.e. $L^{x}$ is positive for $x$ near 0 , and hence we have $\mathbb{N}_{0}$-a.e. that $\left\{x:|x| \leq k^{-1}\right\} \subset \mathcal{R}$ for $k \geq 1$ large. Proposition 5.7 will then imply $\mathbb{N}_{0}$-a.e. for $k \geq 1$ large,

$$
\widetilde{\mathcal{L}}(\kappa)^{\varepsilon_{n}}\left(\left\{x:|x| \leq k^{-1}\right\}\right) \leq \widetilde{\mathcal{L}}(\kappa)^{\varepsilon_{n}}(\mathcal{R})=0 \text { for all } \varepsilon_{n}>0 .
$$

On the other hand, we know that the range of SBM $X$ is compact $\mathbb{N}_{0}$-a.e. by (2.3) and hence by Proposition 5.7 we have $\mathbb{N}_{0}$-a.e. that

$$
\text { for } k \geq 1 \text { large, } \sup _{\varepsilon_{n}>0} \widetilde{\mathcal{L}}(\kappa)^{\varepsilon_{n}}(\{x:|x| \geq k\}) \leq \sup _{\varepsilon_{n}>0} \widetilde{\mathcal{L}}(\kappa)^{\varepsilon_{n}}\left(\mathcal{R}^{>1}\right)=0 .
$$

Combining (5.14), (5.15) and (5.16), we get

$$
\sup _{\varepsilon_{n}>0} \widetilde{\mathcal{L}}(\kappa)^{\varepsilon_{n}}(1)<\infty, \mathbb{N}_{0} \text {-a.e. }
$$

Note (5.16) also implies the tightness of $\left\{\widetilde{\mathcal{L}}(\kappa)^{\varepsilon_{n}}\right\}$ and together with (5.17), we get the relative compactness of $\left\{\widetilde{\mathcal{L}}(\kappa)^{\varepsilon_{n}}\right\}$ by Prohorov's theorem (see, e.g., Theorem 7.8.7 of [1]). By the relative compactness of $\left\{\widetilde{\mathcal{L}}(\kappa)^{\varepsilon_{n}}\right\}$, any subsequence admits a further sequence along which the measures converge to some $\widetilde{\mathcal{L}}(\kappa)$ in the weak topology. It remains to check all limit point coincide which is easy to see by (5.13) since $\mathcal{C}$ is a determining class on $M_{F}\left(\mathbb{R}^{d}\right)$. In conclusion, for any sequence $\varepsilon_{k} \downarrow 0$, we can find a subsequence $\varepsilon_{k_{n}} \downarrow 0$ such that $\mathbb{N}_{0}$-a.e. $\widetilde{\mathcal{L}}(\kappa)^{\varepsilon_{k_{n}}} \rightarrow \widetilde{\mathcal{L}}(\kappa)$, which easily implies that $\widetilde{\mathcal{L}}(\kappa)^{\varepsilon} \stackrel{P}{\rightarrow} \widetilde{\mathcal{L}}(\kappa)$ under $\mathbb{N}_{0}$. The case for $\mathcal{L}^{\lambda} \stackrel{P}{\rightarrow} \mathcal{L}$ under $\mathbb{N}_{0}$ is similar.

After establishing the existence of $\mathcal{L}$ and $\widetilde{\mathcal{L}}(\kappa)$, we continue to show that they differ only up to some constant. It is easy to check that for any $\varepsilon, \lambda>0$ and any $\psi_{m, k} \in \mathcal{C}$,

$$
\begin{aligned}
& \mathbb{N}_{0}\left(\left(K_{4.1} \widetilde{\mathcal{L}}(\kappa)\left(\psi_{m, k}\right)-C_{4.1}(\kappa) \mathcal{L}\left(\psi_{m, k}\right)\right)^{2}\right) \\
& \leq 4 \mathbb{N}_{0}\left(\left(K_{4.1} \widetilde{\mathcal{L}}(\kappa)\left(\psi_{m, k}\right)-K_{4.1} \widetilde{\mathcal{L}}(\kappa)^{\varepsilon}\left(\psi_{m, k}\right)\right)^{2}\right) \\
& +4 \mathbb{N}_{0}\left(\left(K_{4.1} \widetilde{\mathcal{L}}(\kappa)^{\varepsilon}\left(\psi_{m, k}\right)-C_{4.1}(\kappa) \mathcal{L}^{\lambda}\left(\psi_{m, k}\right)\right)^{2}\right) \\
& +4 \mathbb{N}_{0}\left(\left(C_{4.1}(\kappa) \mathcal{L}^{\lambda}\left(\psi_{m, k}\right)-C_{4.1}(\kappa) \mathcal{L}\left(\psi_{m, k}\right)\right)^{2}\right) .
\end{aligned}
$$

By letting $\lambda \rightarrow \infty$ and $\varepsilon \downarrow 0$, we conclude by Corollary 5.2 and Corollary 5.3 that each term on the right-hand side of (5.18) converges to 0 and hence

$$
K_{4.1} \widetilde{\mathcal{L}}(\kappa)\left(\psi_{m, k}\right)=C_{4.1}(\kappa) \mathcal{L}\left(\psi_{m, k}\right), \mathbb{N}_{0} \text {-a.e. }
$$

Take a countable union of null sets to conclude that $\mathbb{N}_{0}$-a.e. for all $m, k \geq 1$, we have $C_{4.1}(\kappa) \mathcal{L}\left(\psi_{m, k}\right)=K_{4.1} \widetilde{\mathcal{L}}(\kappa)\left(\psi_{m, k}\right)$ and so $C_{4.1}(\kappa) \mathcal{L}=K_{4.1} \widetilde{\mathcal{L}}(\kappa)$. Let $c_{1.13}(\kappa)=C_{4.1}(\kappa) K_{4.1}^{-1}$ to see that $\mathbb{N}_{0}$-a.e. we have $\widetilde{\mathcal{L}}(\kappa)=c_{1.13}(\kappa) \mathcal{L}$.

Finally we will show that $\widetilde{\mathcal{L}}(\kappa)$ (and hence $\mathcal{L}$ ) is supported on $\partial \mathcal{R}$. Let $\left\{\varepsilon_{n}\right\}_{n \geq 1}$ be any sequence such that $\mathbb{N}_{0}$-a.e. $\widetilde{\mathcal{L}}(\kappa)^{\varepsilon_{n}} \rightarrow \widetilde{\mathcal{L}}(\kappa)$. By Proposition 5.7 we can fix $\omega$ outside a null set such that $\widetilde{\mathcal{L}}(\kappa)^{\varepsilon_{n}} \rightarrow \widetilde{\mathcal{L}}(\kappa)$ and $\widetilde{\mathcal{L}}(\kappa)^{\varepsilon_{n}}(\mathcal{R}) \rightarrow 0$ hold. It follows that

$$
\widetilde{\mathcal{L}}(\kappa)(\operatorname{Int}(\mathcal{R})) \leq \liminf _{\varepsilon_{n} \downarrow 0} \widetilde{\mathcal{L}}(\kappa)^{\varepsilon_{n}}(\operatorname{Int}(\mathcal{R})) \leq \liminf _{\varepsilon_{n} \downarrow 0} \widetilde{\mathcal{L}}(\kappa)^{\varepsilon_{n}}(\mathcal{R})=0,
$$

where the first inequality is by $\widetilde{\mathcal{L}}(\kappa)^{\varepsilon_{n}} \rightarrow \widetilde{\mathcal{L}}(\kappa)$.

Next by Proposition 5.7 we can take a countable union of null sets and fix $\omega$ outside a null set such that $\widetilde{\mathcal{L}}(\kappa)^{\varepsilon_{n}} \rightarrow \widetilde{\mathcal{L}}(\kappa)$ and $\widetilde{\mathcal{L}}(\kappa)^{\varepsilon_{n}}\left(\mathcal{R}^{>1 / k}\right) \rightarrow 0$ holds for all $k \geq 1$. Then we 
have

$$
\begin{aligned}
\widetilde{\mathcal{L}}(\kappa)\left(\mathcal{R}^{c}\right) & =\widetilde{\mathcal{L}}(\kappa)\left(\bigcup_{k=1}^{\infty} \mathcal{R}^{>1 / k}\right) \leq \sum_{k=1}^{\infty} \widetilde{\mathcal{L}}(\kappa)\left(\mathcal{R}^{>1 / k}\right) \\
& \leq \sum_{k=1}^{\infty} \liminf _{\varepsilon_{n} \downarrow 0} \widetilde{\mathcal{L}}(\kappa)^{\varepsilon_{n}}\left(\mathcal{R}^{>1 / k}\right)=0
\end{aligned}
$$

where the second inequality is by $\widetilde{\mathcal{L}}(\kappa)^{\varepsilon_{n}} \rightarrow \widetilde{\mathcal{L}}(\kappa)$. Therefore we conclude the support of $\widetilde{\mathcal{L}}(\kappa)$ is on $\partial \mathcal{R}$ under $\mathbb{N}_{0}$.

Turning to the case under $\mathbb{P}_{\delta_{0}}$, the above arguments work in an exactly same way as $\mathbb{N}_{0}$ and so we omit the details.

\subsection{On the moments of the boundary local time measure}

In view of Theorems 5.1, 5.4 and Corollaries 5.2, 5.5, we can get the moment measure formulas for $\mathcal{L}$ and $\widetilde{\mathcal{L}}(\kappa)$ and finish the proof of Theorems 1.8 and 1.10 .

Proof of Theorem 1.8. (a) Let $\lambda_{n}$ be the sequence from Theorem 1.3 such that $\mathcal{L}^{\lambda_{n}} \rightarrow \mathcal{L}, \mathbb{N}_{0}$-a.e. For any bounded continuous function $\phi \geq 0$ and any $k \geq 1$, we have $\mathcal{L}^{\lambda_{n}}\left(\phi \cdot \chi_{k}\right) \rightarrow \mathcal{L}\left(\phi \cdot \chi_{k}\right), \mathbb{N}_{0}$-a.e., where $\chi_{k}$ is as in (5.12). Corollary 5.2 will then give that $\mathcal{L}^{\lambda_{n}}\left(\phi \cdot \chi_{k}\right)$ converges in $L^{2}\left(\mathbb{N}_{0}\right)$ to $\mathcal{L}\left(\phi \cdot \chi_{k}\right)$. In particular, by working with the finite measure $\mathbb{N}_{0}\left(\cdot \cap\left\{\mathcal{R} \cap G_{1 / 4 k} \neq \emptyset\right\}\right)$, we have $\mathcal{L}^{\lambda_{n}}\left(\phi \cdot \chi_{k}\right)$ converges in $L^{1}\left(\mathbb{N}_{0}\right)$ to $\mathcal{L}\left(\phi \cdot \chi_{k}\right)$ and so

$$
\begin{aligned}
& \mathbb{N}_{0}\left(\mathcal{L}\left(\phi \cdot \chi_{k}\right)\right)=\lim _{n \rightarrow \infty} \mathbb{N}_{0}\left(\mathcal{L}^{\lambda_{n}}\left(\phi \cdot \chi_{k}\right)\right) \\
= & \lim _{n \rightarrow \infty} \int \phi(x) \chi_{k}(x) \mathbb{N}_{0}\left(\lambda_{n}^{1+\alpha} L^{x} e^{-\lambda_{n} L^{x}}\right) d x=c_{3.8} \int|x|^{-p} \phi(x) \chi_{k}(x) d x,
\end{aligned}
$$

where the second equality is by Fubini's theorem and in the last equality we have used the dominated convergence theorem with Proposition 3.2 and Proposition 3.8. Let $k \rightarrow \infty$ and apply the monotone class theorem to extend (5.20) to any Borel measurable function $\phi$ and the proof follows by $K_{4.1}=c_{3.8}$.

(b) By (4.13), (1.20) follows immediately from (1.19). For the proof of (1.19), we let $\lambda_{n}$ be the sequence such that $\mathcal{L}^{\lambda_{n}} \rightarrow \mathcal{L}, \mathbb{N}_{0}$-a.e. For any bounded continuous function $h \geq 0$ and any $k \geq 1$, we have $\mathbb{N}_{0}$-a.e. that

$$
\left\{\begin{array}{l}
(i) \quad \lim _{n \rightarrow \infty} \mathcal{L}^{\lambda_{n}}\left(\chi_{k}\right) \rightarrow \mathcal{L}\left(\chi_{k}\right) \\
(i i) \quad \lim _{n \rightarrow \infty} \int h\left(x_{1}, x_{2}\right) \chi_{k}\left(x_{1}\right) \chi_{k}\left(x_{2}\right) d \mathcal{L}^{\lambda_{n}}\left(x_{1}\right) d \mathcal{L}^{\lambda_{n}}\left(x_{2}\right) \\
\quad=\int h\left(x_{1}, x_{2}\right) \chi_{k}\left(x_{1}\right) \chi_{k}\left(x_{2}\right) d \mathcal{L}\left(x_{1}\right) d \mathcal{L}\left(x_{2}\right)
\end{array}\right.
$$

Note $h \leq\|h\|_{\infty}$ and so

$$
\left|\int h\left(x_{1}, x_{2}\right) \chi_{k}\left(x_{1}\right) \chi_{k}\left(x_{2}\right) d \mathcal{L}^{\lambda_{n}}\left(x_{1}\right) d \mathcal{L}^{\lambda_{n}}\left(x_{2}\right)\right| \leq\|h\|_{\infty}\left(\mathcal{L}^{\lambda_{n}}\left(\chi_{k}\right)\right)^{2}
$$

Use Corollary 5.2 and (5.21)(i) to get $\mathcal{L}^{\lambda_{n}}\left(\chi_{k}\right)$ converges in $L^{2}\left(\mathbb{N}_{0}\right)$ to $\mathcal{L}\left(\chi_{k}\right)$ and thus we get $\mathbb{N}_{0}\left(\left(\mathcal{L}^{\lambda_{n}}\left(\chi_{k}\right)\right)^{2}\right)$ converges to $\mathbb{N}_{0}\left(\left(\mathcal{L}\left(\chi_{k}\right)\right)^{2}\right)$. Use (5.21)(i) again and work under the finite measure $\mathbb{N}_{0}\left(\cdot \cap\left\{\mathcal{R} \cap G_{1 / 4 k} \neq \emptyset\right\}\right)$ to get $\left\{\left(\mathcal{L}^{\lambda_{n}}\left(\chi_{k}\right)\right)^{2}, n \geq 1\right\}$ is uniformly integrable. By (5.22), the left-hand side term of (5.21)(ii) is also uniformly integrable and hence we 
conclude

$$
\begin{aligned}
\mathbb{N}_{0} & \left(\int h\left(x_{1}, x_{2}\right) \chi_{k}\left(x_{1}\right) \chi_{k}\left(x_{2}\right) d \mathcal{L}\left(x_{1}\right) d \mathcal{L}\left(x_{2}\right)\right) \\
& =\lim _{n \rightarrow \infty} \mathbb{N}_{0}\left(\int h\left(x_{1}, x_{2}\right) \chi_{k}\left(x_{1}\right) \chi_{k}\left(x_{2}\right) d \mathcal{L}^{\lambda_{n}}\left(x_{1}\right) d \mathcal{L}^{\lambda_{n}}\left(x_{2}\right)\right) \\
& =K_{4.1}^{2} \int h\left(x_{1}, x_{2}\right) \chi_{k}\left(x_{1}\right) \chi_{k}\left(x_{2}\right)\left(-U_{1,2}^{\vec{\infty}, \vec{x}}(0)\right) d x_{1} d x_{2},
\end{aligned}
$$

where the last equality is by Theorem 5.1. Let $k \rightarrow \infty$ and apply the monotone class theorem to extend (5.23) to any Borel measurable function.

Proof of Theorem 1.10. The proof of (1.21) and (1.22) follows in a similar way to the above proof of Theorem 1.8 by using Corollary 3.9, Theorem 5.4, Corollary 5.5. (1.23) follows immediately from (1.22), (4.13) and the definitions of $U_{i}^{\vec{\infty}, \vec{x}}$ from (1.16).

\section{Exit measures and zero-one law}

In this section we will give the proof of Theorem 1.6. Our approach to Theorem 1.6 is similar to the proof of Theorem 1.2 in [9]; we utilize exit measures, which will be easy consequences of the following two results. The first result is proved below.

Proposition 6.1. Let $x_{1} \in \mathbb{R}^{d}$ and $r_{0}>0$ satisfy $B_{2 r_{0}}\left(x_{1}\right) \subset S\left(X_{0}\right)^{c}$. If $0<r_{1}<r_{0}$, then $\mathbb{N}_{X_{0}}$-a.e.

$$
\left\{\begin{array}{l}
X_{G_{r_{1}}^{x_{1}}}(1)=0 \text { and } X_{G_{r_{0}}^{x_{1}}}(1)>0 \text { imply } \\
\mathcal{L}\left(B_{r}\left(x_{1}\right)\right)>0 \text { for every } r>r_{1} \text { s.t. } X_{G_{r}^{x_{1}}}(1)>0 .
\end{array}\right.
$$

Corollary 6.2. Let $x_{1} \in \mathbb{R}^{d}$ and $r_{0}>0$ satisfy $B_{2 r_{0}}\left(x_{1}\right) \subset S\left(X_{0}\right)^{c}$. If $0<r_{1}<r_{0}$, then $\mathbb{P}_{X_{0}}$-a.s.

$$
X_{G_{r_{1}}^{x_{1}}}(1)=0 \text { and } X_{G_{r_{0}}^{x_{1}}}(1)>0 \text { imply } \mathcal{L}\left(B_{r_{0}}\left(x_{1}\right)\right)>0 .
$$

Proof. It follows in a similar way to the proof of Proposition 1.6 assuming Proposition 1.7 in [9] by replacing $\operatorname{dim}\left(\partial \mathcal{R} \cap B_{r}\right) \geq d_{f}$ with $\mathcal{L}\left(B_{r}\right)>0$.

Proof of Theorem 1.6. By using Proposition 6.1 and Corollary 6.2, the proof of (1.10) follows in a same way as the proof of Theorem 1.2 of [9]. (1.11) is immediate from (1.10). To see that with $\mathbb{P}_{X_{0}}$-probability one, $\operatorname{Supp}(\mathcal{L})=S\left(X_{0}\right)^{c} \cap \partial \mathcal{R}$, we pick any $x \in S\left(X_{0}\right)^{c} \cap$ $\partial \mathcal{R}$. There is some $\varepsilon>0$ so that $B(x, r) \subset S\left(X_{0}\right)^{c}$ and $B(x, r) \cap \partial \mathcal{R} \neq \emptyset$ for all $0<r<\varepsilon$. Apply (1.10) with $U=B(x, r)$ to see that $\mathcal{L}(B(x, r))>0$ for all $0<r<\varepsilon$ and so conclude $x \in \operatorname{Supp}(\mathcal{L})$, giving $S\left(X_{0}\right)^{c} \cap \partial \mathcal{R} \subset \operatorname{Supp}(\mathcal{L})$. Together with Theorem 1.4 we have $\operatorname{Supp}(\mathcal{L})=S\left(X_{0}\right)^{c} \cap \partial \mathcal{R}, \mathbb{P}_{X_{0}}$-a.s. and the proof is complete.

Now it remains to prove Proposition 6.1. We first state a result that plays the role of Lemma 5.4 in [9].

Lemma 6.3. There is a constant $q_{6.3}>0$ so that if $X_{0}^{\prime} \in M_{F}\left(\mathbb{R}^{d}\right)$ is supported on $\{|x|=r\}$ and $\delta=X_{0}^{\prime}(1)$ satisfies $0<\delta \leq r^{2}$, then

$$
\mathbb{P}_{X_{0}^{\prime}}\left(\mathcal{L}\left(B\left(0, r-\frac{\sqrt{\delta}}{2}\right)\right)>0\right) \geq q_{6.3}
$$

Proof. Define $X_{0}^{(\delta)}(A)=\delta^{-1} X_{0}^{\prime}(\sqrt{\delta} A)$, so that $X_{0}^{(\delta)}$ is supported on $\{|x|=r / \sqrt{\delta}\}$ and has total mass one. By scaling properties of SBM, we may conclude that

$$
\mathbb{P}_{X_{0}^{\prime}}\left(\mathcal{L}\left(B\left(0, r-\frac{\sqrt{\delta}}{2}\right)\right)>0\right)=\mathbb{P}_{X_{0}^{(\delta)}}\left(\mathcal{L}\left(B\left(0, \frac{r}{\sqrt{\delta}}-\frac{1}{2}\right)\right)>0\right)
$$


Now work in our standard set-up for SBM with initial law $X_{0}^{(\delta)}$ so that $X_{t}=\sum_{j \in J} X_{t}\left(W_{j}\right)=\int X_{t}(W) \Xi(d W)$ for all $t>0$, where $\Xi$ is a Poisson point process with intensity $\mathbb{N}_{X_{0}^{(\delta)}}$. For $r \geq \sqrt{\delta}$ define

$$
\begin{aligned}
& \tau_{\rho}\left(W_{j}\right)=\inf \left\{t \geq 0:\left|\hat{W}_{j}(t)\right| \leq \rho\right\}, \\
& U_{\rho}\left(W_{j}\right)=\inf \left\{t \geq 0:\left|\hat{W}_{j}(t)-\hat{W}_{j}(0)\right| \geq \rho\right\}, \\
& \text { and } N_{1}=\sum_{j \in J} 1\left(\tau_{(r / \sqrt{\delta})-(1 / 2)}\left(W_{j}\right)<\infty\right):=\#\left(I_{r, \delta}\right) .
\end{aligned}
$$

Here as usual $\inf \emptyset=\infty$. Then $N_{1}$ is Poisson with mean

$$
\begin{aligned}
m_{r, \delta}:=\mathbb{N}_{X_{0}^{(\delta)}}\left(\tau_{(r / \sqrt{\delta})-(1 / 2)}<\infty\right) & \leq \mathbb{N}_{X_{0}^{(\delta)}}\left(U_{1 / 2}(W)<\infty\right) \\
& =\mathbb{N}_{0}\left(U_{1 / 2}(W)<\infty\right):=\bar{m}<\infty
\end{aligned}
$$

where $X_{0}^{(\delta)}(1)=1$ and translation invariance are used in the equality, and the finiteness of $\bar{m}$ follows from Theorem 1 of [13]. We may assume (by additional randomization) that conditional on $I_{r, \delta},\left\{W_{j}: j \in I_{r, \delta}\right\}$ are iid with law $\mathbb{N}_{X_{0}^{(\delta)}}\left(W \in \cdot \mid \tau_{(r / \sqrt{\delta})-(1 / 2)}<\infty\right)$. Therefore the right-hand side of (6.1) is at least

$$
\begin{gathered}
\mathbb{P}_{X_{0}^{(\delta)}}\left(N_{1}=1\right) \mathbb{N}_{X_{0}^{(\delta)}}\left(\mathcal{L}\left(B\left(0, \frac{r}{\sqrt{\delta}}-\frac{1}{2}\right)\right)>0 \mid \tau_{\frac{r}{\sqrt{\delta}}-\frac{1}{2}}<\infty\right) \\
=\frac{m_{r, \delta} e^{-m_{r, \delta}}}{m_{r, \delta}} \mathbb{N}_{x_{0}}\left(\mathcal{L}\left(B\left(0, \frac{r}{\sqrt{\delta}}-\frac{1}{2}\right)\right)>0\right)
\end{gathered}
$$

where $x_{0}=\left(\frac{r}{\sqrt{\delta}}\right) e_{1}$ and $e_{1}$ is the first unit basis vector. We also have used the facts that spherical symmetry shows we could have taken any $x_{0}$ on the sphere of radius $r / \sqrt{\delta}$ and $\mathcal{L}\left(B\left(0, \frac{r}{\sqrt{\delta}}-\frac{1}{2}\right)\right)=0$ if $\tau_{\frac{r}{\sqrt{\delta}}-\frac{1}{2}}=\infty$ by the fact that $\operatorname{Supp}(\mathcal{L})=\partial \mathcal{R}, \mathbb{N}_{x_{0}}$-a.e. from Corollary 1.7 and translation variance. Now again use translation invariance and spherical symmetry to see that the right side of (6.3) equals

$$
\begin{aligned}
e^{-m_{r, \delta}} \mathbb{N}_{0}\left(\mathcal{L}\left(B\left(x_{0},\left|x_{0}\right|-\frac{1}{2}\right)\right)>0\right) & \geq e^{-\bar{m}} \mathbb{N}_{0}\left(\mathcal{L}\left(B\left(e_{1}, 1 / 2\right)\right)>0\right) \\
& \geq e^{-\bar{m}} \frac{\left(\mathbb{N}_{0}\left(\mathcal{L}\left(B\left(e_{1}, 1 / 2\right)\right)\right)\right)^{2}}{\mathbb{N}_{0}\left(\left(\mathcal{L}\left(B\left(e_{1}, 1 / 2\right)\right)\right)^{2}\right)}
\end{aligned}
$$

where the first inequality follows by (6.2) and $B\left(e_{1}, 1 / 2\right) \subset B\left(x_{0},\left|x_{0}\right|-\frac{1}{2}\right)$ since $x_{0}=\left|x_{0}\right| e_{1}$ and $\left|x_{0}\right| \geq 1$, and the last follows by the second moment method.

Now apply Theorem 1.8 (a) with $\phi(x)=1_{B\left(e_{1}, 1 / 2\right)}(x)$ and Theorem 1.8 (b) with $h\left(x_{1}, x_{2}\right)=1_{B\left(e_{1}, 1 / 2\right)}\left(x_{1}\right) 1_{B\left(e_{1}, 1 / 2\right)}\left(x_{2}\right)$ to get

$$
\mathbb{N}_{0}\left(\mathcal{L}\left(B\left(e_{1}, 1 / 2\right)\right)\right)=K_{4.1} \int_{\left|x-e_{1}\right|<1 / 2}|x|^{-p} d x \geq K_{4.1}\left(\frac{3}{2}\right)^{-p}|B(0,1 / 2)|>0
$$

and

$$
\mathbb{N}_{0}\left(\left(\mathcal{L}\left(B\left(e_{1}, 1 / 2\right)\right)\right)^{2}\right) \leq K_{4.1}^{2} \int_{\left|x_{1}-e_{1}\right|,\left|x_{2}-e_{1}\right|<1 / 2} c_{4.2}\left(2^{p}+2^{p}\right)\left|x_{1}-x_{2}\right|^{2-p} d x_{1} d x_{2}<\infty .
$$

Thus we have shown that the right-hand side of (6.4) has some lower bound $e^{-\bar{m}} c>0$ for some universal constant $c>0$, and so have proved the lemma with $q_{6.3}=e^{-\bar{m}} c$. 
Now we proceed to the proof of Proposition 6.1. Using the setting from Proposition 6.1 , by translation invariance we may assume $x_{1}=0$ and fix $r_{0}>0$ such that

$$
B_{2 r_{0}} \subset S\left(X_{0}\right)^{c} .
$$

Notation. We define $Y_{r}(\cdot)=X_{G_{r_{0}-r}}(\cdot)$ and $\mathcal{E}_{r}=\mathcal{E}_{G_{r_{0}-r}} \vee\left\{\mathbb{N}_{X_{0}}\right.$ - null sets $\}$ for $0 \leq r<r_{0}$. It is not hard to show that $\mathcal{E}_{r}$ is non-decreasing in $r$ (see Section 6 of [9]). Intuitively $\mathcal{E}_{r}$ is the $\sigma$-field generated by the excursions of $W$ in $G_{r_{0}-r}$. By Proposition 2.3 of [15], $Y$ is $\left(\mathcal{E}_{r}\right)$-adapted. Let $\mathcal{E}_{r}^{+}=\mathcal{E}_{r+}$ denote the associated right-continuous filtration. Note Proposition 6.2(b) in [9] gives a cadlag version of $Y_{r}(1)$ which has no negative jumps and is an $\left(\mathcal{E}_{r}^{+}\right)$-supermartingale. In what follows we always work with this cadlag version of $Y_{r}(1)$.

In addition to $\mathbb{N}_{X_{0}}$, we will also work under the probability $Q_{X_{0}}(\cdot)=\mathbb{N}_{X_{0}}\left(\cdot \mid Y_{0}(1)>0\right)$, where (6.5) ensures that $\mathbb{N}_{X_{0}}\left(Y_{0}(1)>0\right)<\infty$. Note that

$$
\text { for any r.v. } Z \geq 0 \text {, and any } r \geq 0, Q_{X_{0}}\left(Z \mid \mathcal{E}_{r}\right)=\mathbb{N}_{X_{0}}\left(Z \mid \mathcal{E}_{r}\right) Q_{X_{0}} \text {-a.s. }
$$

because $\left\{Y_{0}(1)>0\right\} \in \mathcal{E}_{0}$. When conditioning on $\mathcal{E}_{r}$ under $Q_{X_{0}}$, we are adding the slightly larger class of $Q_{X_{0}}$-null sets to $\mathcal{E}_{r}$, but will not record this distinction in our notation. We write $Q_{x_{0}}$ for $Q_{\delta_{x_{0}}}$ as usual.

Let $W$ denote a generic Brownian snake under $\mathbb{N}_{X_{0}}$ and $Q_{X_{0}}$ with the associated "tip process" $\hat{W}(t)$ and excursion length $\sigma$. Define

$$
T_{0}(W)=\inf \left\{r \in\left[0, r_{0}\right): Y_{r}(1)=0\right\} \in\left[0, r_{0}\right], \text { where } \inf \emptyset=r_{0},
$$

and

$$
\hat{T}_{0}(W)=\inf \{|\hat{W}(t)|: 0 \leq t \leq \sigma\}=\inf \{|x|: x \in \mathcal{R}\},
$$

where the last equality holds $\mathbb{N}_{X_{0}}$ by (2.3). Clearly we have $Q_{X_{0}}(\cdot)=\mathbb{N}_{X_{0}}\left(\cdot \mid T_{0}>0\right)$. By Lemma 7.1 of [9], we have

$$
\mathbb{N}_{X_{0}}-\text { a.e. }\left\{T_{0}>0\right\}=\left\{\hat{T}_{0}<r_{0}\right\} \text {, and on this set } \hat{T}_{0}=r_{0}-T_{0} .
$$

Define a sequence of $\left(\mathcal{E}_{r}^{+}\right)$-stopping times by

$$
T_{n^{-1}}=\inf \left\{r \in\left[0, r_{0}\right): Y_{r}(1) \leq 1 / n\right\} \quad\left(\inf \emptyset=r_{0}\right) .
$$

Then

$$
\text { on }\left\{0<T_{0}\right\} \text { (and so } Q_{X_{0}} \text {-a.s.) } T_{n^{-1}} \uparrow T_{0} \text { and } T_{n^{-1}}<T_{0} \text {, }
$$

where the last inequality holds since $Y_{r}(1)$ has no negative jumps. So under $Q_{X_{0}}, T_{0}$ is a predictable stopping time which is announced by $\left\{T_{n^{-1}}\right\}$ and so (see (12.9)(ii) in Chapter VI of [22])

$$
\mathcal{E}_{T_{0}-}^{+}=\vee_{n} \mathcal{E}_{T_{n}}^{+} .
$$

Lemma 6.4. If $X_{0}=\delta_{x_{0}}$ where $\left|x_{0}\right| \geq 2 r_{0}$, then $\mathcal{L}\left(B_{r_{0}}\right) \in \mathcal{E}_{T_{0}^{-}}^{+}$.

Proof. Note Theorem 1.3 implies there is some $\lambda_{n} \rightarrow \infty$ such that $\mathcal{L}^{\lambda_{n}} \rightarrow \mathcal{L}, \mathbb{N}_{x_{0}}$-a.e. by translation invariance. On the other hand, by Theorem 1.8 we have $\mathbb{N}_{x_{0}}\left(\mathcal{L}\left(\partial B_{r_{0}}\right)\right)=0$ and so $\mathbb{N}_{x_{0}}$-a.e., $\mathcal{L}\left(B_{r_{0}}\right)=\lim _{n \rightarrow \infty} \mathcal{L}^{\lambda_{n}}\left(B_{r_{0}}\right)$. As is shown in the proof of Lemma 7.3 in [9], we have $\psi(W) \in \mathcal{E}_{T_{0}^{-}}^{+}$for any Borel map $\psi$ on $C\left(\mathbb{R}^{+}, \mathcal{W}\right)$. Then it follows that $L^{x} \in \mathcal{E}_{T_{0}^{-}}^{+}$ for any $x \in B_{r_{0}}$ and so $\mathcal{L}^{\lambda_{n}}\left(B_{r_{0}}\right) \in \mathcal{E}_{T_{0}^{-}}^{+}$for any $\lambda_{n}>0$, thus proving $\mathcal{L}\left(B_{r_{0}}\right) \in \mathcal{E}_{T_{0}^{-}}^{+}$. 
Proof of Proposition 6.1. Clearly it suffices to fix $x_{0} \in S\left(X_{0}\right)$ and prove the result with $\mathbb{N}_{x_{0}}$ in place of $\mathbb{N}_{X_{0}}$. By translation invariance we may assume $x_{1}=0$, and so $\left|x_{0}\right| \geq 2 r_{0}$. Fix $0<r_{1}<r_{0}$. Assume $0 \leq r<r_{0}$ and $n \in \mathbb{N}$ is large enough so that $r+n^{-1}<r_{0}$. Recall that conditional expectations with respect to $\mathcal{E}_{r}$, under $\mathbb{N}_{x_{0}}$ and $Q_{x_{0}}$, agree $Q_{x_{0}}$-a.s. Therefore up to $Q_{x_{0}}$-null sets, on $\left\{4 n^{-2} \leq Y_{r}(1) \leq\left(r_{0}-r\right)^{2}\right\}\left(\in \mathcal{E}_{r}\right)$ we have

$$
\begin{aligned}
Q_{x_{0}}\left(\mathcal{L}\left(B_{r_{0}}\right)>0 \mid \mathcal{E}_{r}\right) & \geq Q_{x_{0}}\left(\mathcal{L}\left(\bar{B}_{r_{0}-r-n^{-1}}\right)>0 \mid \mathcal{E}_{r}\right) \\
& \geq Q_{x_{0}}\left(\limsup _{k \rightarrow \infty} \mathcal{L}^{\lambda_{k}}\left(\bar{B}_{r_{0}-r-n^{-1}}\right)>0 \mid \mathcal{E}_{r}\right) \\
& =\lim _{m \rightarrow \infty} Q_{x_{0}}\left(\limsup _{k \rightarrow \infty} \mathcal{L}^{\lambda_{k}}\left(\bar{B}_{r_{0}-r-n^{-1}}\right)>m^{-1} \mid \mathcal{E}_{r}\right),
\end{aligned}
$$

where the second inequality is by $\mathcal{L}^{\lambda_{k}} \rightarrow \mathcal{L}$ in $M_{F}$ with the $\left\{\lambda_{k}\right\}$ from Theorem 1.3. The last equality uses the monotone convergence theorem. For each $m \geq 1$ we have

$$
\begin{aligned}
& Q_{x_{0}}\left(\limsup _{k \rightarrow \infty} \mathcal{L}^{\lambda_{k}}\left(\bar{B}_{r_{0}-r-n^{-1}}\right)>m^{-1} \mid \mathcal{E}_{r}\right) \\
& \geq \liminf _{k \rightarrow \infty} Q_{x_{0}}\left(\mathcal{L}^{\lambda_{k}}\left(B_{r_{0}-r-n^{-1}}\right)>m^{-1} \mid \mathcal{E}_{r}\right) \\
& =\liminf _{k \rightarrow \infty} \mathbb{P}_{Y_{r}}\left(\mathcal{L}^{\lambda_{k}}\left(B_{r_{0}-r-n^{-1}}\right)>m^{-1}\right) \\
& \geq \mathbb{P}_{Y_{r}}\left(\liminf _{k \rightarrow \infty} \mathcal{L}^{\lambda_{k}}\left(B_{r_{0}-r-n^{-1}}\right)>m^{-1}\right) \quad(\text { by Fatou's Lemma }) \\
& \geq \mathbb{P}_{Y_{r}}\left(\mathcal{L}\left(B_{r_{0}-r-n^{-1}}\right)>m^{-1}\right) \quad\left(\text { by }\left.\left.\mathcal{L}^{\lambda_{k}}\right|_{\bar{B}_{r_{0}-r-n^{-1}}} \rightarrow \mathcal{L}\right|_{\bar{B}_{r_{0}-r-n^{-1}}}\right)
\end{aligned}
$$

where we have used Proposition 2.1(iii) in the equality and the last inequality is by Theorem 1.4 and by replacing $\left\{\lambda_{k}\right\}$ with a further subsequence which is still denoted by $\left\{\lambda_{k}\right\}$. Combining (6.9) and (6.10), we get up to $Q_{x_{0}}$-null sets, on the event $\left\{4 n^{-2} \leq Y_{r}(1) \leq\left(r_{0}-r\right)^{2}\right\}$ (which is in $\mathcal{E}_{r}$ ), we have

$$
\begin{aligned}
& Q_{x_{0}}\left(\mathcal{L}\left(B_{r_{0}}\right)>0 \mid \mathcal{E}_{r}\right) \geq \liminf _{m \rightarrow \infty} \mathbb{P}_{Y_{r}}\left(\mathcal{L}\left(B_{r_{0}-r-n^{-1}}\right)>m^{-1}\right) \\
= & \mathbb{P}_{Y_{r}}\left(\mathcal{L}\left(B_{r_{0}-r-n^{-1}}\right)>0\right) \geq \mathbb{P}_{Y_{r}}\left(\mathcal{L}\left(B_{r_{0}-r-\left(\sqrt{Y_{r}(1)} / 2\right)}\right)>0\right) \geq q_{6.3},
\end{aligned}
$$

where Lemma 6.3 and the assumed upper bounds on $Y_{r}(1)$ are used in the last inequality, and the assumed lower bound on $Y_{r}(1)$ is used in the next to last inequality. Let $n \rightarrow \infty$ and take limits from above in $r \in \mathbb{Q}_{+}$(recall $Y_{r}(1)$ is cadlag) to conclude that $Q_{x_{0}}$-a.s. $\forall r \in \mathbb{Q} \cap\left(0, r_{0}\right)$,

$$
M_{r}:=Q_{x_{0}}\left(\mathcal{L}\left(B_{r_{0}}\right)>0 \mid \mathcal{E}_{r}^{+}\right) \geq q_{6.3} \text { on }\left\{0<Y_{r}(1)<\left(r_{0}-r\right)^{2}\right\} .
$$

Here $M_{r}$ is a cadlag version of the bounded martingale on the left-hand side. Using right-continuity one can strengthen (6.12) to $Q_{x_{0}}$-a.s. $\forall r \in\left(0, r_{0}\right)$,

$$
M_{r}=Q_{x_{0}}\left(\mathcal{L}\left(B_{r_{0}}\right)>0 \mid \mathcal{E}_{r}^{+}\right) \geq q_{6.3} \text { on }\left\{0<Y_{r}(1)<\left(r_{0}-r\right)^{2}\right\} .
$$

On $\left\{0<T_{0} \leq r_{0}-r_{1}\right\}$, by (6.8) and the lack of negative jumps for $Y_{r}(1)$, we have $Q_{x_{0}}$-a.s. that

$$
\text { for } n \text { large, } T_{n^{-1}} \in\left(0, r_{0}-r_{1}\right) \text { and } Y_{T_{n^{-1}}}(1)=n^{-1}<\left(r_{0}-T_{1 / n}\right)^{2} \text {. }
$$

By Corollary (17.10) in Chapter VI of [22], (6.13), and (6.14), we have $Q_{x_{0}}$-a.s. on $\left\{0<T_{0} \leq r_{0}-r_{1}\right\} \in \mathcal{E}_{T_{0}-}^{+}$,

$$
Q_{x_{0}}\left(\mathcal{L}\left(B_{r_{0}}\right)>0 \mid \mathcal{E}_{T_{0}-}^{+}\right)=\lim _{n \rightarrow \infty} M\left(T_{n^{-1}}\right) \geq q_{6.3} .
$$


Multiplying the above by $1\left(\left\{0<T_{0} \leq r_{0}-r_{1}\right\}\right)$, we see from Lemma 6.4 that $Q_{x_{0}}$-a.s.,

$$
1\left(\left\{\mathcal{L}\left(B_{r_{0}}\right)>0\right\} \cap\left\{0<T_{0} \leq r_{0}-r_{1}\right\}\right) \geq q_{6.3} 1\left(\left\{0<T_{0} \leq r_{0}-r_{1}\right\}\right),
$$

and therefore by (6.7),

$$
r_{1} \leq \hat{T}_{0}<r_{0} \text { implies } \mathcal{L}\left(B_{r_{0}}\right)>0 \quad Q_{x_{0}} \text {-a.s. }
$$

This remains true if we replace $r_{0}$ by any $r \in\left(r_{1}, r_{0}\right]$ since we still have $B_{2 r} \subset S\left(X_{0}\right)^{c}$. Therefore we may fix $\omega$ outside a $Q_{x_{0}}$-null set so that for any $r \in\left(r_{1}, r_{0}\right] \cap \mathbb{Q}, r_{1} \leq \hat{T}_{0}<r$ implies $\mathcal{L}\left(B_{r}\right)>0$. By monotonicity of the conclusion in $r$ this means that $\left\{r_{1} \leq \hat{T}_{0}<r_{0}\right\}$ implies $\mathcal{L}\left(B_{r}\right)>0$ for all $r>\hat{T}_{0}$. This gives Proposition 6.1 under $Q_{x_{0}}$. The result under $\mathbb{N}_{x_{0}}$ is now immediate from the definition of $Q_{x_{0}}$, and $\left\{Y_{0}(1)>0\right\}=\left\{\hat{T}_{0}<r_{0}\right\} \mathbb{N}_{x_{0}}$-a.e. by (6.7).

\section{Change of measure}

Before turning to the proofs of Propositions 4.1, 4.2 and 4.3, we state a result on the change of measure that plays a central role in the proofs. This result is a generalization of Proposition 3.7 where only radially symmetric functions are considered. We implement the ideas there and prove stronger results to deal with non-radial functions.

Let $Y=\left(Y_{s}, s \geq 0\right)$ denote the coordinate variables on $C\left([0, \infty), \mathbb{R}^{d}\right)$ and set $\left(\mathcal{Y}_{t}\right)$ to be the right continuous filtration generated by $Y$. Under the law $P_{x}$ (Wiener measure), $Y$ is a standard $d$-dimensional Brownian motion starting from $x$. Recall $\mu, \nu$ as in (1.13) and recall $\hat{P}_{x}^{(2-2 \nu)}$ is the law under which, $Y$ is the unique solution of

$$
\left\{\begin{array}{l}
Y_{t}=x+\hat{B}_{t}+\int_{0}^{t}(-\nu-\mu) \frac{Y_{s}}{\left|Y_{s}\right|^{2}} d s, \quad t<\tau_{0} \\
Y_{t}=0, \quad t \geq \tau_{0}
\end{array}\right.
$$

where $\tau_{\varepsilon}=\tau_{\varepsilon}^{Y}=\inf \left\{t \geq 0:\left|Y_{t}\right| \leq \varepsilon\right\}$ and $\hat{B}$ is a standard $d$-dimensional Brownian motion under $\hat{P}_{x}^{(2-2 \nu)}$. The upper index $2-2 \nu<0$ on $\hat{P}_{x}^{(2-2 \nu)}$ is to remind us that under $\hat{P}_{x}^{(2-2 \nu)}$, the radial process $\left\{\left|Y_{s}\right|, s \geq 0\right\}$, as we will show later, is a $(2-2 \nu)$-dimensional Bessel process stopped at 0 . Now we proceed to the key proposition for proving the convergence of the second moments.

Proposition 7.1. Let $x \in \mathbb{R}^{d} \backslash\{0\}$ and $0<\varepsilon<|x|$. If $\Phi_{t} \geq 0$ is $\mathcal{Y}_{t}$-adapted, then for any Borel measurable function $g: \mathbb{R}^{d} \rightarrow \mathbb{R}$ such that $P_{x}$-a.s. $\int_{0}^{\tau_{\varepsilon}}\left|g\left(Y_{s}\right)\right| d s<\infty$, we have

$$
\begin{aligned}
& E_{x}\left(1\left(\tau_{\varepsilon}<\infty\right) \Phi_{\tau_{\varepsilon}} \exp \left(-\int_{0}^{\tau_{\varepsilon}} g\left(Y_{s}\right) d s\right)\right) \\
& =\varepsilon^{p}|x|^{-p} \hat{E}_{x}^{(2-2 \nu)}\left(\Phi_{\tau_{\varepsilon}} \exp \left(-\int_{0}^{\tau_{\varepsilon}}\left(g\left(Y_{s}\right)-V^{\infty}\left(Y_{s}\right)\right) d s\right)\right) .
\end{aligned}
$$

Proof. By the monotone convergence theorem we have

$$
\begin{aligned}
I & :=E_{x}\left(1\left(\tau_{\varepsilon}<\infty\right) \Phi_{\tau_{\varepsilon}} \exp \left(-\int_{0}^{\tau_{\varepsilon}} g\left(Y_{s}\right) d s\right)\right) \\
& =\lim _{t \rightarrow \infty} E_{x}\left(1\left(\tau_{\varepsilon} \leq t\right) \Phi_{\tau_{\varepsilon}} \exp \left(-\int_{0}^{\tau_{\varepsilon}} g\left(Y_{s}\right) d s\right)\right) \\
& =\lim _{t \rightarrow \infty} E_{x}\left(1\left(\tau_{\varepsilon} \leq \tau_{\varepsilon} \wedge t\right) \Phi_{\tau_{\varepsilon} \wedge t} \exp \left(-\int_{0}^{\tau_{\varepsilon} \wedge t} g\left(Y_{s}\right) d s\right)\right) .
\end{aligned}
$$

Use Ito's lemma to see that under $P_{x}$,

$$
\log \left|Y_{\tau_{\varepsilon} \wedge t}\right|=\log \left|Y_{0}\right|+\int_{0}^{\tau_{\varepsilon} \wedge t} \frac{Y_{s}}{\left|Y_{s}\right|^{2}} \cdot d Y_{s}+\frac{1}{2} \int_{0}^{\tau_{\varepsilon} \wedge t} \frac{d-2}{\left|Y_{s}\right|^{2}} d s, \forall t \geq 0 .
$$


Recall $\mu, \nu$ as in (1.13) and consider

$$
M_{\varepsilon}(t)=\exp \left(\int_{0}^{t \wedge \tau_{\varepsilon}}(\nu-\mu) \frac{Y_{s}}{\left|Y_{s}\right|^{2}} \cdot d Y_{s}-\frac{1}{2} \int_{0}^{t \wedge \tau_{\varepsilon}} \frac{(\nu-\mu)^{2}}{\left|Y_{s}\right|^{2}} d s\right) .
$$

As one can easily check, $M_{\varepsilon}$ is a martingale under $P_{x}$. Moreover by using (7.4) we can get

$$
M_{\varepsilon}(t)=\frac{\left|Y_{\tau_{\varepsilon} \wedge t}\right|^{\nu-\mu}}{\left|Y_{0}\right|^{\nu-\mu}} \exp \left(-\int_{0}^{\tau_{\varepsilon} \wedge t} \frac{2(4-d)}{\left|Y_{s}\right|^{2}} d s\right) .
$$

An application of Girsanov's theorem (see, e.g., Chapter IV.4 of [12]) implies there is a unique probability $\widetilde{P}_{\varepsilon, x}^{(2+2 \nu)}$ on $C\left([0, \infty), \mathbb{R}^{d}\right)$ so that for any $t \geq 0$,

$$
\left.d \widetilde{P}_{\varepsilon, x}^{(2+2 \nu)}\right|_{\mathcal{Y}_{t}}=\left.\frac{\left|Y_{\tau_{\varepsilon} \wedge t}\right|^{\nu-\mu}}{|x|^{\nu-\mu}} \exp \left(-\int_{0}^{\tau_{\varepsilon} \wedge t} \frac{2(4-d)}{\left|Y_{s}\right|^{2}} d s\right) d P_{x}\right|_{\mathcal{Y}_{t}},
$$

and under $\widetilde{P}_{\varepsilon, x}^{(2+2 \nu)}, Y$ is the unique solution of

$$
Y_{t}=x+\widetilde{B}_{t}+\int_{0}^{\tau_{\varepsilon} \wedge t}(\nu-\mu) \frac{Y_{s}}{\left|Y_{s}\right|^{2}} d s,
$$

(so the drift is stopped when $Y$ hits the ball $\overline{B(0, \varepsilon)}$ ). Here $\widetilde{B}$ is a standard $d$-dimensional Brownian motion with respect to $\widetilde{P}_{\varepsilon, x}^{(2+2 \nu)}$. The upper index $2+2 \nu$ on $\widetilde{P}_{\varepsilon, x}^{(2+2 \nu)}$ is to indicate that the radial process $\left\{\left|Y_{s \wedge \tau_{\varepsilon}}\right|, s \geq 0\right\}$ is a $(2 \nu+2)$-dimensional Bessel process stopped when it hits $\varepsilon>0$ :

$$
\begin{aligned}
\left|Y_{\tau_{\varepsilon} \wedge t}\right|^{2} & =|x|^{2}+\int_{0}^{\tau_{\varepsilon} \wedge t} 2 Y_{s} \cdot\left(d \widetilde{B}_{s}+(\nu-\mu) \frac{Y_{s}}{\left|Y_{s}\right|^{2}} d s\right)+d\left(\tau_{\varepsilon} \wedge t\right) \\
& =|x|^{2}+\int_{0}^{\tau_{\varepsilon} \wedge t} 2\left|Y_{s}\right| \sum_{i=1}^{d} \frac{Y_{s}^{i}}{\left|Y_{s}\right|} d \widetilde{B}_{s}^{i}+(2 \nu+2)\left(\tau_{\varepsilon} \wedge t\right) \\
& =|x|^{2}+\int_{0}^{\tau_{\varepsilon} \wedge t} 2\left|Y_{s}\right| d \widetilde{\beta}_{s}+(2 \nu+2)\left(\tau_{\varepsilon} \wedge t\right),
\end{aligned}
$$

where the last equality follows since $\widetilde{\beta}_{t}=\sum_{i=1}^{d} \frac{Y_{t}^{i}}{\left|Y_{t}\right|} \widetilde{B}_{t}^{i}$ is a one-dimensional Brownian motion under $\widetilde{P}_{\varepsilon, x}^{(2+2 \nu)}$. Therefore $\left\{\left|Y_{s \wedge \tau_{\varepsilon}}\right|^{2}, s \geq 0\right\}$ satisfies the SDE of a stopped square Bessel process of dimension $2+2 \nu$ and so $\left\{\left|Y_{s \wedge \tau_{\varepsilon}}\right|, s \geq 0\right\}$ is a stopped $(2+2 \nu)$-dimensional Bessel process (see Chp. XI of [21] for the definition of square Bessel process and its connection with Bessel process). It follows that

$$
\widetilde{P}_{\varepsilon, x}^{(2+2 \nu)}\left(\tau_{\varepsilon}<\infty\right)=\frac{\varepsilon^{2 \nu}}{|x|^{2 \nu}}
$$

Now apply (7.7) to see that (7.3) becomes

$$
\begin{aligned}
I & =\lim _{t \rightarrow \infty} \widetilde{E}_{\varepsilon, x}^{(2+2 \nu)}\left(1_{\left(\tau_{\varepsilon} \leq \tau_{\varepsilon} \wedge t\right)} \Phi_{\tau_{\varepsilon} \wedge t} \exp \left(-\int_{0}^{\tau_{\varepsilon} \wedge t}\left(g\left(Y_{s}\right)-V^{\infty}\left(Y_{s}\right)\right) d s\right) \frac{\left|Y_{\tau_{\varepsilon} \wedge t}\right|^{\mu-\nu}}{|x|^{\mu-\nu}}\right) \\
& =\lim _{t \rightarrow \infty} \widetilde{E}_{\varepsilon, x}^{(2+2 \nu)}\left(1\left(\tau_{\varepsilon} \leq t\right) \Phi_{\tau_{\varepsilon}} \exp \left(-\int_{0}^{\tau_{\varepsilon}}\left(g\left(Y_{s}\right)-V^{\infty}\left(Y_{s}\right)\right) d s\right) \frac{\left|Y_{\tau_{\varepsilon}}\right|^{\mu-\nu}}{|x|^{\mu-\nu}}\right) \\
& =\frac{\varepsilon^{\mu-\nu}}{|x|^{\mu-\nu}} \widetilde{E}_{\varepsilon, x}^{(2+2 \nu)}\left(1\left(\tau_{\varepsilon}<\infty\right) \Phi_{\tau_{\varepsilon}} \exp \left(-\int_{0}^{\tau_{\varepsilon}}\left(g\left(Y_{s}\right)-V^{\infty}\left(Y_{s}\right)\right) d s\right)\right) \\
& =\frac{\varepsilon^{p}}{|x|^{p}} \widetilde{E}_{\varepsilon, x}^{(2+2 \nu)}\left(\left.\Phi_{\tau_{\varepsilon}} \exp \left(-\int_{0}^{\tau_{\varepsilon}}\left(g\left(Y_{s}\right)-V^{\infty}\left(Y_{s}\right)\right) d s\right)\right|_{\tau_{\varepsilon}}<\infty\right),
\end{aligned}
$$

where we have used the monotone convergence theorem in the next to last equality and the last equality follows from (7.10) and $p=\mu+\nu$.

We interrupt the proof of the proposition for another auxiliary result. 
Lemma 7.2. For any $\varepsilon>0$ and $|x|>\varepsilon$, we have the law of $\left\{Y_{s \wedge \tau_{\varepsilon}}, s \geq 0\right\}$ conditioning on $\left\{\tau_{\varepsilon}<\infty\right\}$ under $\widetilde{P}_{\varepsilon, x}^{(2+2 \nu)}$ is equal to the law of $\left\{Y_{s \wedge \tau_{\varepsilon}}, s \geq 0\right\}$ under $\hat{P}_{x}^{(2-2 \nu)}$ defined as in (7.1).

Proof. For any $0<t_{1}<\cdots<t_{n}$ and any bounded Borel functions $\phi_{i}: \mathbb{R}^{d} \rightarrow \mathbb{R}, 1 \leq i \leq n$, we use (7.10) to get

$$
\begin{aligned}
J & :=\widetilde{E}_{\varepsilon, x}^{(2+2 \nu)}\left(\prod_{i=1}^{n} \phi_{i}\left(Y_{t_{i} \wedge \tau_{\varepsilon}}\right) \mid \tau_{\varepsilon}<\infty\right)=\widetilde{E}_{\varepsilon, x}^{(2+2 \nu)}\left(\prod_{i=1}^{n} \phi_{i}\left(Y_{t_{i} \wedge \tau_{\varepsilon}}\right) 1_{\left\{\tau_{\varepsilon}<\infty\right\}}\right) \cdot \frac{|x|^{2 \nu}}{\varepsilon^{2 \nu}} \quad \text { (7.12) } \\
& =\widetilde{E}_{\varepsilon, x}^{(2+2 \nu)}\left(\prod_{i=1}^{n} \phi_{i}\left(Y_{t_{i} \wedge \tau_{\varepsilon}}\right) \widetilde{P}_{\varepsilon, Y_{t_{n} \wedge \tau_{\varepsilon}}}^{(2+2 \nu)}\left(\tau_{\varepsilon}<\infty\right)\right) \cdot \frac{|x|^{2 \nu}}{\varepsilon^{2 \nu}}=\widetilde{E}_{\varepsilon, x}^{(2+2 \nu)}\left(\prod_{i=1}^{n} \phi_{i}\left(Y_{t_{i} \wedge \tau_{\varepsilon}}\right) \frac{|x|^{2 \nu}}{\left|Y_{t_{n} \wedge \tau_{\varepsilon}}\right|^{2 \nu}}\right),
\end{aligned}
$$

where the second last equality is by the strong Markov property of $Y$. Similar to the derivation of (7.7) using (7.4), (7.5) and (7.6), by replacing $\nu$ with $-\nu$ in (7.5) and (7.6), another application of Girsanov's theorem implies there is a unique probability $\hat{P}_{\varepsilon, x}^{(2-2 \nu)}$ on $C\left([0, \infty), \mathbb{R}^{d}\right)$ so that for any $t \geq 0$,

$$
\left.d \hat{P}_{\varepsilon, x}^{(2-2 \nu)}\right|_{\mathcal{Y}_{t}}=\left.\frac{\left|Y_{\tau_{\varepsilon} \wedge t}\right|^{-\nu-\mu}}{|x|^{-\nu-\mu}} \exp \left(-\int_{0}^{\tau_{\varepsilon} \wedge t} \frac{2(4-d)}{\left|Y_{s}\right|^{2}} d s\right) d P_{x}\right|_{\mathcal{Y}_{t}},
$$

and under $\hat{P}_{\varepsilon, x}^{(2-2 \nu)}, Y$ is the unique solution of

$$
Y_{t}=x+\hat{B}_{t}+\int_{0}^{\tau_{\varepsilon} \wedge t}(-\nu-\mu) \frac{Y_{s}}{\left|Y_{s}\right|^{2}} d s
$$

(so again the drift is stopped when $Y$ hits the ball $\overline{B(0, \varepsilon)}$ ). Here $\hat{B}$ is a standard $d$ dimensional Brownian motion with respect to $\hat{P}_{\varepsilon, x}^{(2-2 \nu)}$. Combining (7.7) and (7.13), we can get

$$
\left.\hat{P}_{\varepsilon, x}^{(2-2 \nu)}\right|_{\mathcal{Y}_{t}}=\left.\frac{|x|^{2 \nu}}{\left|Y_{\tau_{\varepsilon} \wedge t}\right|^{2 \nu}} \widetilde{P}_{\varepsilon, x}^{(2+2 \nu)}\right|_{\mathcal{Y}_{t}} .
$$

Now apply (7.15) in (7.12) to see that

$$
J=\hat{E}_{\varepsilon, x}^{(2-2 \nu)}\left(\prod_{i=1}^{n} \phi_{i}\left(Y_{t_{i} \wedge \tau_{\varepsilon}}\right)\right)=\hat{E}_{x}^{(2-2 \nu)}\left(\prod_{i=1}^{n} \phi_{i}\left(Y_{t_{i} \wedge \tau_{\varepsilon}}\right)\right),
$$

where the last equality follows since one can easily check that $\left\{Y_{t \wedge \tau_{\varepsilon}}, t \geq 0\right\}$ under $\hat{P}_{\varepsilon, x}^{(2-2 \nu)}$ is equal in law to that under $\hat{P}_{x}^{(2-2 \nu)}$ (see (7.1) and (7.14)). So the proof is complete.

Returning to the proof of Proposition 7.1, we apply the above lemma in (7.11) to conclude

$$
I=\frac{\varepsilon^{p}}{|x|^{p}} \hat{E}_{x}^{(2-2 \nu)}\left(\Phi_{\tau_{\varepsilon}} \exp \left(-\int_{0}^{\tau_{\varepsilon}}\left(g\left(Y_{s}\right)-V^{\infty}\left(Y_{s}\right)\right) d s\right)\right),
$$

and the proof is complete.

One can show (as for (7.9)) that the radial process $\left\{\left|Y_{s \wedge \tau_{0}}\right|, s \geq 0\right\}$ under $\hat{P}_{x}^{(2-2 \nu)}$ is a $(2-2 \nu)$-dimensional Bessel process stopped at 0. By applying Lemma 7.2 to the radial process $\left\{\left|Y_{s \wedge \tau_{\varepsilon}}\right|, s \geq 0\right\}$, we can get following "well-known" result on Bessel process (see Corollary 2.3 of Lawler [14]).

Corollary 7.3. For $\delta \in \mathbb{R}$, let $\left(\rho_{t}\right)$ denote a $\delta$-dimensional Bessel process starting from $r>0$ under $P_{r}^{(\delta)}$. For any $\gamma>0$ and any $\varepsilon>0$ such that $r>\varepsilon$, we have the law of $\left\{\rho_{s \wedge \tau_{\varepsilon}}, s \geq 0\right\}$ conditioning on $\left\{\tau_{\varepsilon}<\infty\right\}$ under $P_{r}^{(2+2 \gamma)}$ is equal to the law of $\left\{\rho_{s \wedge \tau_{\varepsilon}}, s \geq 0\right\}$ under $P_{r}^{(2-2 \gamma)}$. 


\section{Proofs of Proposition 4.1 and Proposition 4.3(i)}

In this section we will give the proof of Proposition 4.1 and Proposition 4.3(i). Recall the definitions of $U^{\vec{\lambda}, \vec{x}, \vec{\varepsilon}}, V^{\vec{\lambda}, \vec{x}}$ and $W^{\vec{\lambda}, \vec{x}, \varepsilon}$ from Section 4 .

Throughout the rest of this paper, we note when dealing with $U^{\vec{\lambda}, \vec{x}, \vec{\varepsilon}}$, we will fix $\lambda_{1}, \lambda_{2}>0$ and let $\varepsilon_{1}, \varepsilon_{2}$ converge to 0 . For $V^{\vec{\lambda}, \vec{x}}$ we will let $\lambda_{1}, \lambda_{2}$ converge to infinity; for $W^{\lambda, \vec{x}, \varepsilon}$ we will fix $\lambda_{2}>0$ and let $\lambda_{1}$ converge to infinity and $\varepsilon$ converge to 0 .

\subsection{Preliminaries}

Lemma 8.1. For any $x_{1} \neq x_{2}$ and $x \neq x_{1}, x_{2}$, we have

$$
\lim _{\varepsilon_{1}, \varepsilon_{2} \downarrow 0} U^{\vec{\lambda}, \vec{x}, \vec{\varepsilon}}(x)=\lim _{\lambda_{1}, \lambda_{2} \rightarrow \infty} V^{\vec{\lambda}, \vec{x}}(x)=\lim _{\lambda_{1} \rightarrow \infty, \varepsilon \downarrow 0} W^{\vec{\lambda}, \vec{x}, \varepsilon}(x)=V^{\infty, \vec{x}}(x),
$$

where $V^{\infty, \vec{x}}(x)$ is as in (1.15).

Proof. This result follows intuitively from (1.42) and more details for the proof can be found in Appendix B.

Use $1-a b \leq(1-a)+(1-b)$ for all $0 \leq a, b \leq 1$ to see that for all $x$ so that $\left|x-x_{i}\right|>\varepsilon_{i}, i=1,2$,

$$
\begin{aligned}
U^{\vec{\lambda}, \vec{x}, \vec{\varepsilon}}(x) & \leq \sum_{i=1}^{2} \mathbb{N}_{x}\left(1-\exp \left(-\lambda_{i} \frac{X_{G_{\varepsilon_{i}}^{x_{i}}}(1)}{\varepsilon_{i}^{2}}\right) 1\left(X_{G_{\varepsilon_{i} / 2}^{x_{i}}}=0\right)\right) \\
& =\sum_{i=1}^{2} \mathbb{N}_{x}\left(1-\exp \left(-\left(\lambda_{i}+4 U^{\infty, 1}(2)\right) \frac{X_{G_{\varepsilon_{i}}^{x_{i}}}(1)}{\varepsilon_{i}^{2}}\right)\right) \\
& =U^{\widetilde{\lambda}_{1} \varepsilon_{1}^{-2}, \varepsilon_{1}}\left(x-x_{1}\right)+U^{\widetilde{\lambda}_{2} \varepsilon_{2}^{-2}, \varepsilon_{2}}\left(x-x_{2}\right),
\end{aligned}
$$

where the first equality follows in a similar way to the derivation of (3.20) and the last is by (3.18) and by letting

$$
\widetilde{\lambda}_{i}:=\lambda_{i}+4 U^{\infty, 1}(2), i=1,2 .
$$

Next we apply $1-a b \geq(1-a) \vee(1-b), \forall 0 \leq a, b \leq 1$ to see that for all $x$ so that $\left|x-x_{i}\right|>\varepsilon_{i}, i=1,2$,

$$
U^{\vec{\lambda}, \vec{x}, \vec{\varepsilon}}(x) \geq U^{\widetilde{\lambda}_{1} \varepsilon_{1}^{-2}, \varepsilon_{1}}\left(x-x_{1}\right) \vee U^{\widetilde{\lambda}_{2} \varepsilon_{2}^{-2}, \varepsilon_{2}}\left(x-x_{2}\right) .
$$

Similar to the above derivations, one can also show that for all $x \neq x_{1}, x_{2}$,

$$
V^{\lambda_{1}}\left(x-x_{1}\right) \vee V^{\lambda_{2}}\left(x-x_{2}\right) \leq V^{\vec{\lambda}, \vec{x}}(x) \leq V^{\lambda_{1}}\left(x-x_{1}\right)+V^{\lambda_{2}}\left(x-x_{2}\right),
$$

and for all $x \neq x_{1}$ and $\left|x-x_{2}\right|>\varepsilon$,

$$
\left\{\begin{array}{l}
W^{\vec{\lambda}, \vec{x}, \varepsilon}(x) \leq V^{\lambda_{1}}\left(x-x_{1}\right)+U^{\widetilde{\lambda}_{2} \varepsilon^{-2}, \varepsilon}\left(x-x_{2}\right), \\
W^{\vec{\lambda}, \vec{x}, \varepsilon}(x) \geq V^{\lambda_{1}}\left(x-x_{1}\right) \vee U^{\widetilde{\lambda}_{2} \varepsilon^{-2}, \varepsilon}\left(x-x_{2}\right) .
\end{array}\right.
$$

By (4.1) of [9] we have $4 U^{\infty, 1}(2) \geq 4 V^{\infty}(2)=\lambda_{d}$ and so $\widetilde{\lambda}_{i} \geq \lambda_{d}$. Then it follows from (4.17) of [8] that

$$
U^{\widetilde{\lambda}_{i} \varepsilon_{i}^{-2}, \varepsilon_{i}}(x) \geq V^{\infty}(x), \text { for all }|x| \geq \varepsilon_{i} \text { for } i=1,2 .
$$

Together with (8.3), we have for all $x$ so that $\left|x-x_{i}\right| \geq \varepsilon_{i}, i=1,2$,

$$
U^{\vec{\lambda}, \vec{x}, \vec{\varepsilon}}(x) \geq V^{\infty}\left(x-x_{1}\right) \vee V^{\infty}\left(x-x_{2}\right),
$$


and by (8.5) we have for all $x \neq x_{1}$ and $\left|x-x_{2}\right|>\varepsilon$,

$$
W^{\vec{\lambda}, \vec{x}, \varepsilon}(x) \geq V^{\lambda_{1}}\left(x-x_{1}\right) \vee V^{\infty}\left(x-x_{2}\right) .
$$

Fix $x_{1} \neq x_{2}$ and $x \neq x_{1}, x_{2}$. Let $\left(B_{t}\right)$ denote a $d$-dimentional Brownian motion starting from $x$ under $P_{x}$. Let $r_{\lambda_{i}}=\lambda_{0} \lambda_{i}^{-\frac{1}{4-d}}, i=1,2$, where $\lambda_{0}$ will be chosen to be some fixed large constant below. Set $T_{r_{\lambda}}=T_{r_{\lambda_{1}}}^{1} \wedge T_{r_{\lambda_{2}}}^{2}$ where $T_{r_{\lambda_{i}}}^{i}=\inf \left\{t \geq 0:\left|B_{t}-x_{i}\right| \leq r_{\lambda_{i}}\right\}$ for $i=1,2$. Let $\lambda_{1}, \lambda_{2}>0$ be large so that

$$
0<4\left(r_{\lambda_{1}} \vee r_{\lambda_{2}}\right) \leq \min \left\{\left|x-x_{1}\right|,\left|x-x_{2}\right|,\left|x_{1}-x_{2}\right|\right\}
$$

The following result is from Lemma 9.4 of [19].

Lemma 8.2. For any $t>0$, we have for $i=1,2$,

$$
V_{i}^{\vec{\lambda}, \vec{x}}(x)=E_{x}\left(V_{i}^{\vec{\lambda}, \vec{x}}\left(B\left(t \wedge T_{r_{\lambda}}\right)\right) \exp \left(-\int_{0}^{t \wedge T_{r_{\lambda}}} V^{\vec{\lambda}, \vec{x}}\left(B_{s}\right) d s\right)\right) .
$$

Lemma 8.3. Let $G=G_{\varepsilon_{1}}^{x_{1}} \cap G_{\varepsilon_{2}}^{x_{2}}$. Then $U^{\vec{\lambda}, \vec{x}, \vec{\varepsilon}}$ is a $C^{2}$ function on $G$ and solves

$$
\Delta U^{\vec{\lambda}, \vec{x}, \vec{\varepsilon}}=\left(U^{\vec{\lambda}, \vec{x}, \vec{\varepsilon}}\right)^{2} \text { on } G \text {. }
$$

Proof. The proof follows in a similar way to that of Lemma S.1.1 of [10] and will be given in Appendix B.

Set $T_{2 \varepsilon_{i}}^{i}=\inf \left\{t \geq 0:\left|B_{t}-x_{i}\right| \leq 2 \varepsilon_{i}\right\}, i=1,2$ and $T_{\varepsilon}=T_{2 \varepsilon_{1}}^{1} \wedge T_{2 \varepsilon_{2}}^{2}$. Let $\varepsilon_{1}, \varepsilon_{2}>0$ be small so that $0<4\left(\varepsilon_{1} \vee \varepsilon_{2}\right)<\min \left\{\left|x_{1}-x\right|,\left|x_{2}-x\right|,\left|x_{1}-x_{2}\right|\right\}$.

Lemma 8.4. For any $t>0$, we have for $i=1,2$,

$$
U_{i}^{\vec{\lambda}, \vec{x}, \vec{\varepsilon}}(x)=E_{x}\left(U_{i}^{\vec{\lambda}, \vec{x}, \vec{\varepsilon}}\left(B\left(t \wedge T_{\varepsilon}\right)\right) \exp \left(-\int_{0}^{t \wedge T_{\varepsilon}} U^{\vec{\lambda}, \vec{x}, \vec{\varepsilon}}\left(B_{s}\right) d s\right)\right) .
$$

Proof. By using Lemma 8.3, the proof is similar to the derivation of Lemma 8.2.

Lemma 8.5. Let $G=\left\{x: x \neq x_{1}\right\} \cap G_{\varepsilon}^{x_{2}}$. Then $W^{\vec{\lambda}, \vec{x}, \varepsilon}$ is a $C^{2}$ function on $G$ and solves

$$
\Delta W^{\vec{\lambda}, \vec{x}, \varepsilon}=\left(W^{\vec{\lambda}, \vec{x}, \varepsilon}\right)^{2} \text { on } G .
$$

Proof. It follows in a similar manner to the proof of Lemma 8.3.

Let $r_{\lambda_{1}}=\lambda_{0} \lambda_{1}^{-\frac{1}{4-d}}$ where $\lambda_{0}$ will be chosen to be some fixed large constant below. Set $T_{\lambda_{1}, \varepsilon}=T_{r_{\lambda_{1}}}^{1} \wedge T_{2 \varepsilon}^{2}$ where $T_{r_{\lambda_{1}}}^{1}=\inf \left\{t \geq 0:\left|B_{t}-x_{1}\right| \leq r_{\lambda_{1}}\right\}$ and $T_{2 \varepsilon}^{2}=\inf \{t \geq 0$ : $\left.\left|B_{t}-x_{2}\right| \leq 2 \varepsilon\right\}$. Let $\lambda_{1}>0$ large and $\varepsilon>0$ small such that

$$
0<4\left(r_{\lambda_{1}} \vee \varepsilon\right)<\min \left\{\left|x_{1}-x\right|,\left|x_{2}-x\right|,\left|x_{1}-x_{2}\right|\right\}
$$

Lemma 8.6. For any $t>0$, we have for $i=1,2$,

$$
W_{i}^{\vec{\lambda}, \vec{x}, \varepsilon}(x)=E_{x}\left(W_{i}^{\vec{\lambda}, \vec{x}, \varepsilon}\left(B\left(t \wedge T_{\lambda_{1}, \varepsilon}\right)\right) \exp \left(-\int_{0}^{t \wedge T_{\lambda_{1}, \varepsilon}} W^{\vec{\lambda}, \vec{x}, \varepsilon}\left(B_{s}\right) d s\right)\right) .
$$

Proof. By using Lemma 8.5, the proof follows in a similar way to that of Lemma 8.2. 


\subsection{Proofs of Proposition 4.1 and Proposition 4.3(i)}

Given the similarities of the proofs of Propositions 4.1(i), 4.1(ii) and 4.3(i), we will only give the proof of Proposition 4.1(ii) here and other proofs can be found in Appendix C.

Proof of Proposition 4.1(ii). By symmetry it suffices to consider the case $i=1$. Recall Lemma 8.4 to get

$$
\frac{1}{\varepsilon_{1}^{p-2}} U_{1}^{\vec{\lambda}, \vec{x}, \vec{\varepsilon}}(x)=\frac{1}{\varepsilon_{1}^{p-2}} \lim _{t \rightarrow \infty} E_{x}\left(U_{1}^{\vec{\lambda}, \vec{x}, \vec{\varepsilon}}\left(B\left(t \wedge T_{\varepsilon}\right)\right) \exp \left(-\int_{0}^{t \wedge T_{\varepsilon}} U^{\vec{\lambda}, \vec{x}, \vec{\varepsilon}}\left(B_{s}\right) d s\right)\right)
$$

where $T_{\varepsilon}=T_{2 \varepsilon_{1}}^{1} \wedge T_{2 \varepsilon_{2}}^{2}$ and $T_{2 \varepsilon_{i}}^{i}=\inf \left\{t \geq 0:\left|B_{t}-x_{i}\right| \leq 2 \varepsilon_{i}\right\}$ for $i=1,2$. By (5.3), we have $U_{1}^{\vec{\lambda}, \vec{x}, \vec{\varepsilon}}(x) \rightarrow 0$ as $|x| \rightarrow \infty$ and $U_{1}^{\vec{\lambda}, \vec{x}, \vec{\varepsilon}}\left(B\left(t \wedge T_{\varepsilon}\right)\right)$ is uniformly bounded for all $t \geq 0$. Apply the dominated convergence theorem to see that

$$
\begin{aligned}
& \frac{1}{\varepsilon_{1}^{p-2}} U_{1}^{\vec{\lambda}, \vec{x}, \vec{\varepsilon}}(x)=\frac{1}{\varepsilon_{1}^{p-2}} E_{x}\left(1_{\left\{T_{\varepsilon}<\infty\right\}} U_{1}^{\vec{\lambda}, \vec{x}, \vec{\varepsilon}}\left(B\left(T_{\varepsilon}\right)\right) \exp \left(-\int_{0}^{T_{\varepsilon}} U^{\vec{\lambda}, \vec{x}, \vec{\varepsilon}}\left(B_{s}\right) d s\right)\right) \\
& =\sum_{i=1}^{2} E_{x}\left(1_{\left\{T_{2 \varepsilon_{i}}^{i}<\infty\right\}} 1_{\left\{T_{2 \varepsilon_{i}}^{i}<T_{2 \varepsilon_{3-i}}^{3-i}\right\}} \frac{1}{\varepsilon_{1}^{p-2}} U_{1}^{\vec{\lambda}, \vec{x}, \vec{\varepsilon}}\left(B\left(T_{2 \varepsilon_{i}}^{i}\right)\right) \exp \left(-\int_{0}^{T_{2 \varepsilon_{i}}^{i}} U^{\vec{\lambda}, \vec{x}, \vec{\varepsilon}}\left(B_{s}\right) d s\right)\right) \\
& :=I_{1}+I_{2} .
\end{aligned}
$$

We first deal with $I_{2}$. Note in the integrand of $I_{2}$ we may assume that $\left|B\left(T_{2 \varepsilon_{2}}^{2}\right)-x_{2}\right|=2 \varepsilon_{2}$ and so for $\varepsilon_{2}>0$ small we have $\left|x_{1}-B\left(T_{2 \varepsilon_{2}}^{2}\right)\right|>\Delta / 2$ where $\Delta=\left|x_{1}-x_{2}\right|$. Apply (5.3) with $x=B\left(T_{2 \varepsilon_{2}}^{2}\right)$ to get

$$
\frac{1}{\varepsilon_{1}^{p-2}} U_{1}^{\vec{\lambda}, \vec{x}, \vec{\varepsilon}}\left(B\left(T_{2 \varepsilon_{2}}^{2}\right)\right) \leq\left|B\left(T_{2 \varepsilon_{2}}^{2}\right)-x_{1}\right|^{-p} \leq \Delta^{-p} 2^{p} .
$$

Let $\tau_{r}=\inf \left\{t \geq 0:\left|B_{t}\right| \leq r\right\}$ and use the above and (8.7) to see that $I_{2}$ becomes

$$
\begin{aligned}
I_{2} & \leq 2^{p} \Delta^{-p} E_{x}\left(1_{\left\{T_{2 \varepsilon_{2}}^{2}<\infty\right\}} 1_{\left\{T_{2 \varepsilon_{2}}^{2}<T_{2 \varepsilon_{1}}^{1}\right\}} \exp \left(-\int_{0}^{T_{2 \varepsilon_{2}}^{2}} U^{\vec{\lambda}, \vec{x}, \vec{\varepsilon}}\left(B_{s}\right) d s\right)\right) \\
& \leq 2^{p} \Delta^{-p} E_{x-x_{2}}\left(1_{\left\{\tau_{2 \varepsilon_{2}}<\infty\right\}} \exp \left(-\int_{0}^{\tau_{2 \varepsilon_{2}}} V^{\infty}\left(B_{s}\right) d s\right)\right) \\
& =2^{p} \Delta^{-p}\left(2 \varepsilon_{2} /\left|x-x_{2}\right|\right)^{p} \rightarrow 0 \text { as } \varepsilon_{2} \downarrow 0,
\end{aligned}
$$

where in the last equality we have used Proposition 7.1 with $g=V^{\infty}$.

Now we will turn to $I_{1}$. Let $\left(Y_{t}, t \geq 0\right)$ be the $d$-dimensional coordinate process under Wiener measure, $P_{x}$. By slightly abusing the notation, we set $\tau_{r}=\tau_{r}^{Y}=\inf \left\{t \geq 0:\left|Y_{t}\right| \leq\right.$ $r$ for any $r>0$, and set

$$
T_{2 \varepsilon_{2}}^{\prime}=T_{2 \varepsilon_{2}}^{\prime, Y}=\inf \left\{t \geq 0:\left|Y_{t}-\left(x_{2}-x_{1}\right)\right| \leq 2 \varepsilon_{2}\right\} .
$$

Then use translation invariance of $Y$ to get

$$
I_{1}=E_{x-x_{1}}\left(1_{\left\{\tau_{2 \varepsilon_{1}}<\infty\right\}} 1_{\left\{\tau_{2 \varepsilon_{1}}<T_{2 \varepsilon_{2}}^{\prime}\right\}} \frac{1}{\varepsilon_{1}^{p-2}} U_{1}^{\vec{\lambda}, \vec{x}, \vec{\varepsilon}}\left(Y_{\tau_{2 \varepsilon_{1}}}+x_{1}\right) \exp \left(-\int_{0}^{\tau_{2 \varepsilon_{1}}} U^{\vec{\lambda}, \vec{x}, \vec{\varepsilon}}\left(Y_{s}+x_{1}\right) d s\right)\right) .
$$

Recall that $\hat{P}_{x}^{(2-2 \nu)}$ is the law of $Y$ starting from $x$ such that $Y$ satisfies the SDE as 
in (7.1). Apply Proposition 7.1 with $g(\cdot)=U^{\vec{\lambda}, \vec{x}, \vec{\varepsilon}}\left(\cdot+x_{1}\right)$ in the above to get

$$
\begin{aligned}
I_{1}=\frac{\left(2 \varepsilon_{1}\right)^{p}}{\left|x-x_{1}\right|^{p}} \hat{E}_{x-x_{1}}^{(2-2 \nu)}\left(1_{\left\{\tau_{2 \varepsilon_{1}}<T_{2 \varepsilon_{2}}^{\prime}\right\}} \frac{1}{\varepsilon_{1}^{p-2}} U_{1}^{\vec{\lambda}, \vec{x}, \vec{\varepsilon}}\left(Y_{\tau_{2 \varepsilon_{1}}}+x_{1}\right)\right. \\
\left.\times \exp \left(-\int_{0}^{\tau_{2 \varepsilon_{1}}}\left(U^{\vec{\lambda}, \vec{x}, \vec{\varepsilon}}\left(Y_{s}+x_{1}\right)-V^{\infty}\left(Y_{s}\right)\right) d s\right)\right) \\
=\frac{2^{p}}{\left|x-x_{1}\right|^{p}} \hat{E}_{x-x_{1}}^{(2-2 \nu)}\left(\left[1_{\left\{\tau_{2 \varepsilon_{1}}<T_{2 \varepsilon_{2}}^{\prime}\right\}}\right] \times\left[\varepsilon_{1}^{2} U_{1}^{\vec{\lambda}, \vec{x}, \vec{\varepsilon}}\left(Y_{\tau_{2 \varepsilon_{1}}}+x_{1}\right)\right]\right. \\
\times\left[\exp \left(-\int_{0}^{\tau_{2 \varepsilon_{1}}}\left(U^{\vec{\lambda}, \vec{x}, \vec{\varepsilon}}\left(Y_{s}+x_{1}\right)-U^{\widetilde{\lambda}_{1} \varepsilon_{1}^{-2}, \varepsilon_{1}}\left(Y_{s}\right)\right) d s\right)\right] \\
\times \\
\left.\quad\left[\exp \left(-\int_{0}^{\tau_{2 \varepsilon_{1}}}\left(U^{\widetilde{\lambda}_{1} \varepsilon_{1}^{-2}, \varepsilon_{1}}\left(Y_{s}\right)-V^{\infty}\left(Y_{s}\right)\right) d s\right)\right]\right) \\
:=\frac{2^{p}}{\left|x-x_{1}\right|^{p}} \hat{E}_{x-x_{1}}^{(2-2 \nu)}\left(\left[J_{1}\right]\left[J_{2}\right]\left[J_{3}\right]\left[J_{4}\right]\right),
\end{aligned}
$$

where $\widetilde{\lambda}_{1}$ is as in (8.2) and we have ordered the fours terms in square brackets as $J_{1}, \ldots, J_{4}$.

We first consider $J_{2}$. Recall (4.7) and use translation invariance to get

$$
\begin{aligned}
J_{2}= & \mathbb{N}_{Y_{\tau_{2 \varepsilon_{1}}}+x_{1}}\left(X_{G_{\varepsilon_{1}}^{x_{1}}}(1) \prod_{i=1}^{2} \exp \left(-\lambda_{i} \frac{X_{G_{\varepsilon_{i}}^{x_{i}}}(1)}{\varepsilon_{i}^{2}}\right) 1\left(X_{G_{\varepsilon_{i} / 2}^{x_{i}}}=0\right)\right) \\
=\mathbb{N}_{Y_{\tau_{2 \varepsilon_{1}}}} & \left(X_{G_{\varepsilon_{1}}}(1) \exp \left(-\lambda_{1} \frac{X_{G_{\varepsilon_{1}}}(1)}{\varepsilon_{1}^{2}}\right) 1\left(X_{G_{\varepsilon_{1} / 2}}=0\right)\right. \\
& \left.\times \exp \left(-\lambda_{2} \frac{X_{G_{\varepsilon_{2}}^{x_{2}-x_{1}}}(1)}{\varepsilon_{2}^{2}}\right) 1\left(X_{G_{\varepsilon_{2} / 2}^{x_{2}-x_{1}}}=0\right)\right) .
\end{aligned}
$$

By the scaling of Brownian snake and its exit measure under the excursion measure $\mathbb{N}_{x}$ (see, e.g., the proof of Proposition V.9 in [16]), we have

$$
\begin{aligned}
J_{2}=\varepsilon_{1}^{-2} \mathbb{N}_{Y_{\tau_{2} \varepsilon_{1}} / \varepsilon_{1}}\left(\varepsilon_{1}^{2} X_{G_{1}}(1) \exp \left(-\lambda_{1} X_{G_{1}}(1)\right) 1\left(X_{G_{1 / 2}}=0\right)\right. \\
\left.\quad \times \exp \left(-\lambda_{2} \frac{X_{G_{\varepsilon_{2} / \varepsilon_{1}}^{\left(x_{2}-x_{1}\right) / \varepsilon_{1}}}(1)}{\left(\varepsilon_{2} / \varepsilon_{1}\right)^{2}}\right) 1\left(X_{G_{\varepsilon_{2} / 2 \varepsilon_{1}}^{\left(x_{2}-x_{1}\right) / \varepsilon_{1}}}=0\right)\right) \\
\stackrel{\text { law }}{=} \mathbb{N}_{Y_{\tau_{2}}}\left(X_{G_{1}}(1) \exp \left(-\lambda_{1} X_{G_{1}}(1)\right) 1\left(X_{G_{1 / 2}}=0\right)\right. \\
\left.\quad \times \exp \left(-\lambda_{2} \frac{X_{G_{\varepsilon_{2} / \varepsilon_{1}}^{\left(x_{2}-x_{1}\right) / \varepsilon_{1}}}(1)}{\left(\varepsilon_{2} / \varepsilon_{1}\right)^{2}}\right) 1\left(X_{G_{\varepsilon_{2} / 2 \varepsilon_{1}}^{\left(x_{2}-x_{1}\right) / \varepsilon_{1}}}=0\right)\right),
\end{aligned}
$$

where the last equality is by the scaling of $Y$. Note for any $K>0$, we have

$$
\left|\frac{x_{2}-x_{1}}{\varepsilon_{1}}\right|-\frac{\varepsilon_{2}}{\varepsilon_{1}} \geq \frac{\left|x_{2}-x_{1}\right| / 2}{\varepsilon_{1}}>K \text { for } \varepsilon_{1}, \varepsilon_{2} \text { small enough, }
$$

and so by (2.3) and (2.4) we conclude $\mathbb{N}_{Y_{\tau_{2}}}$-a.e.

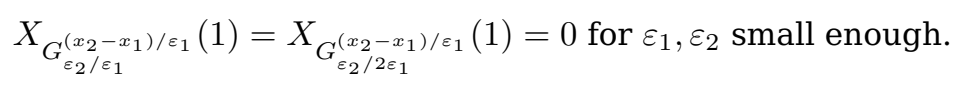


Therefore an application of the dominated convergence theorem will give us

$$
\begin{aligned}
& \lim _{\varepsilon_{1}, \varepsilon_{2} \downarrow 0} \mathbb{N}_{Y_{\tau_{2}}}\left(X_{G_{1}}(1) \exp \left(-\lambda_{1} X_{G_{1}}(1)\right) 1\left(X_{G_{1 / 2}}=0\right)\right. \\
&\left.\quad \times \exp \left(-\lambda_{2} \frac{X_{G_{\varepsilon_{2} / \varepsilon_{1}}^{\left(x_{2}-x_{1}\right) / \varepsilon_{1}}}(1)}{\left(\varepsilon_{2} / \varepsilon_{1}\right)^{2}}\right) 1\left(X_{G_{\varepsilon_{2} / 2 \varepsilon_{1}}^{\left(x_{2}-x_{1}\right) / \varepsilon_{1}}}=0\right)\right) \\
&=\mathbb{N}_{Y_{\tau_{2}}}\left(X_{G_{1}}(1) e^{-\lambda_{1} X_{G_{1}}(1)} 1\left(X_{G_{1 / 2}}=0\right)\right) \\
&=\mathbb{N}_{2 e_{1}}\left(X_{G_{1}}(1) e^{-\lambda_{1} X_{G_{1}}(1)} 1\left(X_{G_{1 / 2}}=0\right)\right)=U_{1}^{\widetilde{\lambda}_{1}, 1}(2),
\end{aligned}
$$

where the next to last equality is by spherical symmetry and $e_{1}$ is the first unit basis vector. In the last equality we have used (3.20), (3.19) with $\varepsilon=1, x=2 e_{1}$ and (8.2). In view of (8.17) and (8.18), we have proved

$$
J_{2}=\varepsilon_{1}^{2} U_{1}^{\vec{\lambda}, \vec{x}, \vec{\varepsilon}}\left(Y_{\tau_{2 \varepsilon_{1}}}+x_{1}\right) \rightarrow U_{1}^{\widetilde{\lambda}_{1}, 1}(2) \text { in distribution as } \varepsilon_{1}, \varepsilon_{2} \downarrow 0 \text {. }
$$

Since $U_{1}^{\widetilde{\lambda}_{1}, 1}(2)$ is a constant, we conclude that under $\hat{P}_{x-x_{1}}^{(2-2 \nu)}$,

$$
J_{2}=\varepsilon_{1}^{2} U_{1}^{\vec{\lambda}, \vec{x}, \vec{\varepsilon}}\left(Y_{\tau_{2 \varepsilon_{1}}}+x_{1}\right) \rightarrow U_{1}^{\widetilde{\lambda}_{1}, 1}(2) \text { in probability as } \varepsilon_{1}, \varepsilon_{2} \downarrow 0 \text {. }
$$

We continue to show that with $\hat{P}_{x-x_{1}}^{(2-2 \nu)}$-probability one,

$$
J_{1}=1_{\left\{\tau_{2 \varepsilon_{1}}<T_{2 \varepsilon_{2}}^{\prime}\right\}} \rightarrow 1 \text { as } \varepsilon_{1}, \varepsilon_{2} \downarrow 0,
$$

and

$$
\begin{aligned}
J_{3}=\exp (- & \left.\int_{0}^{\tau_{2 \varepsilon_{1}}}\left(U^{\vec{\lambda}, \vec{x}, \vec{\varepsilon}}\left(Y_{s}+x_{1}\right)-U^{\widetilde{\lambda}_{1} \varepsilon_{1}^{-2}, \varepsilon_{1}}\left(Y_{s}\right)\right) d s\right) \\
& \rightarrow \exp \left(-\int_{0}^{\tau_{0}}\left(V^{\vec{\infty}, \vec{x}}\left(Y_{s}+x_{1}\right)-V^{\infty}\left(Y_{s}\right)\right) d s\right) \text { as } \varepsilon_{1}, \varepsilon_{2} \downarrow 0 .
\end{aligned}
$$

Since the drift of $\left\{Y_{t}, t \geq 0\right\}$ as in (7.1) is bounded up to time $\tau_{\varepsilon}$ for any $\varepsilon>0$ and since Brownian motion in $d \geq 2$ does not hit points, we conclude by Girsanov's theorem (recall (7.13)) that $\left\{Y_{t}, t \geq \overline{0}\right\}$ does not hit the point $x_{1}-x_{2} \neq 0$ and so with $\hat{P}_{x-x_{1}}^{(2-2 \nu)}$ probability one,

$$
\exists \delta(\omega)>0 \text { so that }\left|Y_{s}-\left(x_{2}-x_{1}\right)\right|>\delta \text { for all } 0 \leq s \leq \tau_{0},
$$

which implies (recall (8.15))

$$
T_{2 \varepsilon_{2}}^{\prime}=\infty \text { for all } 0<\varepsilon_{2}<\delta(\omega) / 2, \quad \hat{P}_{x-x_{1}}^{(2-2 \nu)} \text {-a.s. }
$$

Since $\tau_{0}$ under $\hat{P}_{x-x_{1}}^{(2-2 \nu)}$ is the hitting time $\tau_{0}$ of a $(2-2 \nu)$-dimensional Bessel process, it follows that with $\hat{P}_{x-x_{1}}^{(2-2 \nu)}$-probability one (see, e.g., Exercise (1.33) in Chp. XI of [21])

$$
\tau_{0}<\infty, \quad \hat{P}_{x-x_{1}}^{(2-2 \nu)} \text {-a.s. }
$$

Therefore by (8.23) we have (8.20).

Fix $\omega$ outside a null set such that both (8.22) and (8.24) hold. For all $0<\varepsilon_{1}, \varepsilon_{2}<$ $\delta(\omega) / 2$, we have

$$
\left|Y_{s}\right| \geq 2 \varepsilon_{1} \text { and }\left|Y_{s}-\left(x_{2}-x_{1}\right)\right|>\delta>2 \varepsilon_{2}, \text { for all } 0<s<\tau_{2 \varepsilon_{1}} .
$$


Now apply (8.1) with $Y_{s}+x_{1}$ in place of $x$ to get

$$
\begin{aligned}
& \left(U^{\vec{\lambda}, \vec{x}, \vec{\varepsilon}}\left(Y_{s}+x_{1}\right)-U^{\widetilde{\lambda}_{1} \varepsilon_{1}^{-2}, \varepsilon_{1}}\left(Y_{s}\right)\right) 1_{\left\{0<s<\tau_{2 \varepsilon_{1}}\right\}} \\
& \leq U^{\widetilde{\lambda}_{2} \varepsilon_{2}^{-2}, \varepsilon_{2}}\left(Y_{s}-\left(x_{2}-x_{1}\right)\right) 1_{\left\{0<s<\tau_{2 \varepsilon_{1}}\right\}} \leq U^{\widetilde{\lambda}_{2} \varepsilon_{2}^{-2}, \varepsilon_{2}}(\delta) 1_{\left\{0<s<\tau_{0}\right\}},
\end{aligned}
$$

where in the last inequality we have used (8.25) and the fact that $r \mapsto U^{\lambda, \varepsilon}(r)$ is decreasing from Lemma 3.2(b) of [19]. Corollary 4.3 of [9] gives us

$$
U^{\widetilde{\lambda}_{2}, 1}(x) \leq U^{\infty, 1}(x) \leq 3(4-d)|x|^{-2}, \forall|x|>1 \text { large }
$$

By scaling of $U^{\lambda, \varepsilon}$ from (1.38), we have for $\varepsilon_{2}>0$ small,

$$
U^{\tilde{\lambda}_{2} \varepsilon_{2}^{-2}, \varepsilon_{2}}(\delta)=\varepsilon_{2}^{-2} U^{\tilde{\lambda}_{2}, 1}\left(\delta / \varepsilon_{2}\right) \leq 3(4-d) \delta^{-2} \leq 6 \delta^{-2} .
$$

Combining (8.26) and (8.27), we have for $\varepsilon_{2}>0$ small,

$$
\left(U^{\vec{\lambda}, \vec{x}, \vec{\varepsilon}}\left(Y_{s}+x_{1}\right)-U^{\widetilde{\lambda}_{1} \varepsilon_{1}^{-2}, \varepsilon_{1}}\left(Y_{s}\right)\right) 1_{\left\{0<s<\tau_{2 \varepsilon_{1}}\right\}} \leq 6 \delta^{-2} 1_{\left\{0<s<\tau_{0}\right\}} .
$$

Since we have $\tau_{0}(\omega)<\infty$ by (8.24), we conclude the left-hand side term of (8.28) is bounded by an integrable bound. By (4.38) of [8] we have

$$
\lim _{\varepsilon \downarrow 0} U^{\lambda \varepsilon^{-2}, \varepsilon}(x)=V^{\infty}(x), \forall x \neq 0 \text {, for any } \lambda>0 .
$$

Now use the dominated convergence theorem with Lemma 8.1, (8.28) and (8.29) to see that with $\hat{P}_{x-x_{1}}^{(2-2 \nu)}$-probability one,

$$
\lim _{\varepsilon_{1}, \varepsilon_{2} \downarrow 0} \int_{0}^{\tau_{2 \varepsilon_{1}}}\left(U^{\vec{\lambda}, \vec{x}, \vec{\varepsilon}}\left(Y_{s}+x_{1}\right)-U^{\widetilde{\lambda}_{1} \varepsilon_{1}^{-2}, \varepsilon_{1}}\left(Y_{s}\right)\right) d s=\int_{0}^{\tau_{0}}\left(V^{\vec{\infty}, \vec{x}}\left(Y_{s}+x_{1}\right)-V^{\infty}\left(Y_{s}\right)\right) d s,
$$

thus proving (8.21) holds.

Combine (8.19), (8.20) and (8.21) to see that under $\hat{P}_{x-x_{1}}^{(2-2 \nu)}$, we have

$$
J_{1} J_{2} J_{3} \rightarrow U_{1}^{\widetilde{\lambda}_{1}, 1}(2) \exp \left(-\int_{0}^{\tau_{0}}\left(V^{\vec{\infty}, \vec{x}}\left(Y_{s}+x_{1}\right)-V^{\infty}\left(Y_{s}\right)\right) d s\right)
$$

in probability as $\varepsilon_{1}, \varepsilon_{2} \downarrow 0$.

Recall (5.3) to see that

$$
J_{2}=\varepsilon_{1}^{2} U_{1}^{\vec{\lambda}, \vec{x}, \vec{\varepsilon}}\left(Y_{\tau_{2 \varepsilon_{1}}}+x_{1}\right) \leq \varepsilon_{1}^{p}\left|Y_{\tau_{2 \varepsilon_{1}}}\right|^{-p}=2^{-p}
$$

and together with (8.3) we have $0 \leq J_{1} J_{2} J_{3} \leq 2^{-p}, \hat{P}_{x-x_{1}}^{(2-2)}$-a.s. Recalling $J_{4}$ as in (8.16), we have $0 \leq J_{4} \leq 1$ by (8.6). By (1.27) and the definition of $V^{\vec{\infty}, \vec{x}}$ as in (1.15), we have

$$
V^{\vec{\infty}, \vec{x}}(x) \geq V^{\infty}\left(x-x_{1}\right) \vee V^{\infty}\left(x-x_{2}\right), \quad \forall x \neq x_{1}, x_{2}
$$

Now use (8.30) and the bounded convergence theorem to see that

$$
\begin{aligned}
& \mid \hat{E}_{x-x_{1}}^{(2-2 \nu)}\left(J_{1} J_{2} J_{3} J_{4}\right)-\hat{E}_{x-x_{1}}^{(2-2 \nu)}\left(U_{1}^{\widetilde{\lambda}_{1}, 1}(2)\right. \\
& \left.\times \exp \left(-\int_{0}^{\tau_{0}}\left(V^{\vec{\infty}, \vec{x}}\left(Y_{s}+x_{1}\right)-V^{\infty}\left(Y_{s}\right)\right) d s\right) \times J_{4}\right) \mid \\
& \leq \hat{E}_{\left|x-x_{1}\right|}^{(2-2 \nu)}\left(\left|J_{1} J_{2} J_{3}-U_{1}^{\tilde{\lambda}_{1}, 1}(2) \exp \left(-\int_{0}^{\tau_{0}}\left(V^{\vec{\infty}, \vec{x}}\left(Y_{s}+x_{1}\right)-V^{\infty}\left(Y_{s}\right)\right) d s\right)\right|\right) \\
& \rightarrow 0 \text { as } \varepsilon_{1}, \varepsilon_{2} \downarrow 0 \text {. }
\end{aligned}
$$


In view of (8.16), we conclude

$$
\begin{aligned}
& \lim _{\varepsilon_{1}, \varepsilon_{2} \downarrow 0} I_{1}=\frac{2^{p} U_{1}^{\tilde{\lambda}_{1}, 1}(2)}{\left|x-x_{1}\right|^{p}} \\
& \times \lim _{\varepsilon_{1}, \varepsilon_{2} \downarrow 0} \hat{E}_{x-x_{1}}^{(2-2 \nu)}\left(\exp \left(-\int_{0}^{\tau_{0}}\left(V^{\vec{\infty}, \vec{x}}\left(Y_{s}+x_{1}\right)-V^{\infty}\left(Y_{s}\right)\right) d s\right) \times J_{4}\right),
\end{aligned}
$$

provided we can show the limit on the right-hand side exists. We claim that there is some constant $C\left(\widetilde{\lambda}_{1}\right)>0$ such that

$$
\begin{aligned}
& \lim _{\varepsilon_{1} \downarrow 0} \hat{E}_{x-x_{1}}^{(2-2 \nu)}\left(\exp \left(-\int_{0}^{\tau_{0}}\left(V^{\vec{\infty}, \vec{x}}\left(Y_{s}+x_{1}\right)-V^{\infty}\left(Y_{s}\right)\right) d s\right) \times J_{4}\right) \\
& =C\left(\widetilde{\lambda}_{1}\right) \hat{E}_{x-x_{1}}^{(2-2 \nu)}\left(\exp \left(-\int_{0}^{\tau_{0}}\left(V^{\vec{\infty}, \vec{x}}\left(Y_{s}+x_{1}\right)-V^{\infty}\left(Y_{s}\right)\right) d s\right)\right) .
\end{aligned}
$$

It will then follow from (8.12), (8.14), (8.33) and (8.34) that

$$
\begin{aligned}
\lim _{\varepsilon_{1}, \varepsilon_{2} \downarrow 0} & \frac{1}{\varepsilon_{1}^{p-2}} U_{1}^{\vec{\lambda}, \vec{x}, \vec{\varepsilon}}(x)=2^{p} U_{1}^{\widetilde{\lambda}_{1}, 1}(2) C\left(\widetilde{\lambda}_{1}\right) \\
& \left|x-x_{1}\right|^{-p} \hat{E}_{x-x_{1}}^{(2-2 \nu)}\left(\exp \left(-\int_{0}^{\tau_{0}}\left(V^{\vec{\infty}, \vec{x}}\left(Y_{s}+x_{1}\right)-V^{\infty}\left(Y_{s}\right)\right) d s\right)\right),
\end{aligned}
$$

and the proof is complete by letting $C_{4.1}\left(\lambda_{1}\right)=2^{p} U_{1}^{\widetilde{\lambda}_{1}, 1}(2) C\left(\widetilde{\lambda}_{1}\right)$.

It remains to prove (8.34). First by the monotone convergence theorem and (8.32), we have

$$
\begin{aligned}
\lim _{\delta \downarrow 0} \hat{E}_{x-x_{1}}^{(2-2 \nu)}( & \exp \left(-\int_{0}^{\tau_{\delta}}\left(V^{\vec{\infty}, \vec{x}}\left(Y_{s}+x_{1}\right)-V^{\infty}\left(Y_{s}\right)\right) d s\right) \\
& \left.-\exp \left(-\int_{0}^{\tau_{0}}\left(V^{\vec{\infty}, \vec{x}}\left(Y_{s}+x_{1}\right)-V^{\infty}\left(Y_{s}\right)\right) d s\right)\right)=0 .
\end{aligned}
$$

Since $0 \leq J_{4} \leq 1$ for any $\varepsilon_{1}>0$, it follows from monotonicity and (8.32) that

$$
\begin{aligned}
& \mid \hat{E}_{x-x_{1}}^{(2-2 \nu)}\left(\exp \left(-\int_{0}^{\tau_{\delta}}\left(V^{\vec{\infty}, \vec{x}}\left(Y_{s}+x_{1}\right)-V^{\infty}\left(Y_{s}\right)\right) d s\right) \times J_{4}\right) \\
& -\hat{E}_{x-x_{1}}^{(2-2 \nu)}\left(\exp \left(-\int_{0}^{\tau_{0}}\left(V^{\vec{\infty}, \vec{x}}\left(Y_{s}+x_{1}\right)-V^{\infty}\left(Y_{s}\right)\right) d s\right) \times J_{4}\right) \mid \\
& \leq \hat{E}_{x-x_{1}}^{(2-2 \nu)}\left(\exp \left(-\int_{0}^{\tau_{\delta}}\left(V^{\vec{\infty}, \vec{x}}\left(Y_{s}+x_{1}\right)-V^{\infty}\left(Y_{s}\right)\right) d s\right)\right. \\
& \left.\quad-\exp \left(-\int_{0}^{\tau_{0}}\left(V^{\vec{\infty}, \vec{x}}\left(Y_{s}+x_{1}\right)-V^{\infty}\left(Y_{s}\right)\right) d s\right)\right) \rightarrow 0 \text { as } \delta \downarrow 0
\end{aligned}
$$

uniformly for all $\varepsilon_{1}>0$ by (8.35). Fixing any $\delta>0$, we will show that

$$
\begin{aligned}
& \lim _{\varepsilon_{1} \downarrow 0} \hat{E}_{x-x_{1}}^{(2-2 \nu)}\left(\exp \left(-\int_{0}^{\tau_{\delta}}\left(V^{\vec{\infty}, \vec{x}}\left(Y_{s}+x_{1}\right)-V^{\infty}\left(Y_{s}\right)\right) d s\right) \times J_{4}\right) \\
& =C\left(\widetilde{\lambda}_{1}\right) \hat{E}_{x-x_{1}}^{(2-2 \nu)}\left(\exp \left(-\int_{0}^{\tau_{\delta}}\left(V^{\vec{\infty}, \vec{x}}\left(Y_{s}+x_{1}\right)-V^{\infty}\left(Y_{s}\right)\right) d s\right)\right) .
\end{aligned}
$$

Then one can easily conclude from (8.35), (8.36) and (8.37) that (8.34) holds.

It remains to prove (8.37). Recall $\left(\rho_{s}\right)$ is a $\gamma$-dimensional Bessel process starting from $r>0$ under $P_{r}^{(\gamma)}$ and let $\tau_{\varepsilon}=\tau_{\varepsilon}^{\rho}=\inf \left\{t \geq 0: \rho_{t} \leq \varepsilon\right\}$. Lemma 4.5 of [8] implies that for any $\lambda>0$, there is some constant $0<C_{8.38}(\lambda)<\infty$ so that for all $x \neq 0$,

$$
\lim _{\varepsilon \downarrow 0} E_{|x|}^{(2+2 \nu)}\left(\exp \left(-\int_{0}^{\tau_{2 \varepsilon}}\left(U^{\lambda \varepsilon^{-2}, \varepsilon}-V^{\infty}\right)\left(\rho_{s}\right) d s\right) \mid \tau_{2 \varepsilon}<\infty\right)=C_{8.38}(\lambda) .
$$


For $0<\varepsilon_{1}<\delta / 2$ we apply the strong Markov property of $\left(Y_{s}, s \geq 0\right)$ to get

$$
\begin{aligned}
\hat{E}_{x-x_{1}}^{(2-2 \nu)}\left(\exp \left(-\int_{0}^{\tau_{\delta}}\left(V^{\vec{\infty}, \vec{x}}\left(Y_{s}+x_{1}\right)-V^{\infty}\left(Y_{s}\right)\right) d s\right)\right. \\
\left.\times \exp \left(-\int_{0}^{\tau_{2 \varepsilon_{1}}}\left(U^{\widetilde{\lambda}_{1} \varepsilon_{1}^{-2}, \varepsilon_{1}}\left(Y_{s}\right)-V^{\infty}\left(Y_{s}\right)\right) d s\right)\right) \\
=\hat{E}_{x-x_{1}}^{(2-2 \nu)}\left(\exp \left(-\int_{0}^{\tau_{\delta}}\left(V^{\vec{\infty}, \vec{x}}\left(Y_{s}+x_{1}\right)-V^{\infty}\left(Y_{s}\right)\right) d s\right)\right. \\
\times \exp \left(-\int_{0}^{\tau_{\delta}}\left(U^{\widetilde{\lambda}_{1} \varepsilon_{1}^{-2}, \varepsilon_{1}}\left(Y_{s}\right)-V^{\infty}\left(Y_{s}\right)\right) d s\right) \\
\left.\times \hat{E}_{Y_{\tau_{\delta}}}^{(2-2 \nu)}\left(\exp \left(-\int_{0}^{\tau_{2 \varepsilon_{1}}}\left(U^{\widetilde{\lambda}_{1} \varepsilon_{1}^{-2}, \varepsilon_{1}}\left(Y_{s}\right)-V^{\infty}\left(Y_{s}\right)\right) d s\right)\right)\right) .
\end{aligned}
$$

For the last term on the right-hand side of (8.39), we can use the fact that under $\hat{P}_{x}^{(2-2 \nu)},\left\{\left|Y_{s \wedge \tau_{2 \varepsilon_{1}}}\right|, s \geq 0\right\}$ is a stopped $(2-2 \nu)$-dimensional Bessel process and then use Corollary 7.3 to get

$$
\begin{aligned}
& \hat{E}_{Y_{\tau_{\delta}}}^{(2-2 \nu)}\left(\exp \left(-\int_{0}^{\tau_{2 \varepsilon_{1}}}\left(U^{\widetilde{\lambda}_{1} \varepsilon_{1}^{-2}, \varepsilon_{1}}\left(Y_{s}\right)-V^{\infty}\left(Y_{s}\right)\right) d s\right)\right) \\
& =E_{\left|Y_{\tau_{\delta}}\right|}^{(2-2 \nu)}\left(\exp \left(-\int_{0}^{\tau_{2 \varepsilon_{1}}}\left(U^{\widetilde{\lambda}_{1} \varepsilon_{1}^{-2}, \varepsilon_{1}}\left(\rho_{s}\right)-V^{\infty}\left(\rho_{s}\right)\right) d s\right)\right) \\
& =E_{\delta}^{(2+2 \nu)}\left(\exp \left(-\int_{0}^{\tau_{2 \varepsilon_{1}}}\left(U^{\widetilde{\lambda}_{1} \varepsilon_{1}^{-2}, \varepsilon_{1}}\left(\rho_{s}\right)-V^{\infty}\left(\rho_{s}\right)\right) d s\right) \mid \tau_{2 \varepsilon_{1}}<\infty\right) \\
& \rightarrow C_{8.38}\left(\widetilde{\lambda}_{1}\right) \text { as } \varepsilon_{1} \downarrow 0,
\end{aligned}
$$

where the last is by (8.38). Next since $\delta>0$ is fixed, by (8.29) it follows that with $\hat{P}_{x-x_{1}}^{(2-2 \nu)}$-probability one,

$$
\lim _{\varepsilon_{1} \downarrow 0} \exp \left(-\int_{0}^{\tau_{\delta}}\left(U^{\widetilde{\lambda}_{1} \varepsilon_{1}^{-2}, \varepsilon_{1}}\left(Y_{s}\right)-V^{\infty}\left(Y_{s}\right)\right) d s\right)=1 .
$$

In view of (8.32) and (8.6), with $\hat{P}_{x-x_{1}}^{(2-2 \nu)}$-probability one, for any $\varepsilon_{1}>0$ we have

$$
\begin{aligned}
& \exp \left(-\int_{0}^{\tau_{\delta}}\left(V^{\vec{\infty}, \vec{x}}\left(Y_{s}+x_{1}\right)-V^{\infty}\left(Y_{s}\right)\right) d s\right) \\
& \times \exp \left(-\int_{0}^{\tau_{\delta}}\left(U^{\widetilde{\lambda}_{1} \varepsilon_{1}^{-2}, \varepsilon_{1}}\left(Y_{s}\right)-V^{\infty}\left(Y_{s}\right)\right) d s\right) \\
& \times \hat{E}_{Y_{\tau_{\delta}}}^{(2-2 \nu)}\left(\exp \left(-\int_{0}^{\tau_{2 \varepsilon_{1}}}\left(U^{\widetilde{\lambda}_{1} \varepsilon_{1}^{-2}, \varepsilon_{1}}\left(Y_{s}\right)-V^{\infty}\left(Y_{s}\right)\right) d s\right)\right) \leq 1
\end{aligned}
$$

Combine (8.40), (8.41) and (8.42) to see that the integrand in (8.39) converges pointwise a.s. as $\varepsilon_{1} \downarrow 0$ and is bounded by 1 . Therefore we apply the bounded convergence theorem to conclude

$$
\begin{aligned}
& \lim _{\varepsilon_{1} \downarrow 0} \hat{E}_{x-x_{1}}^{(2-2 \nu)}\left(\exp \left(-\int_{0}^{\tau_{\delta}}\left(V^{\vec{\infty}, \vec{x}}\left(Y_{s}+x_{1}\right)-V^{\infty}\left(Y_{s}\right)\right) d s\right) \times J_{4}\right) \\
& =C_{8.38}\left(\widetilde{\lambda}_{1}\right) \hat{E}_{x-x_{1}}^{(2-2 \nu)}\left(\exp \left(-\int_{0}^{\tau_{\delta}}\left(V^{\vec{\infty}, \vec{x}}\left(Y_{s}+x_{1}\right)-V^{\infty}\left(Y_{s}\right)\right) d s\right)\right),
\end{aligned}
$$

and the proof of (8.37) is complete.

\section{Convergence of the second moments}

In this section we will give the proofs of Proposition 4.2 and Proposition 4.3(ii). 


\subsection{Preliminaries}

Lemma 9.1. For any $\lambda_{1}, \lambda_{2}, \varepsilon_{1}, \varepsilon_{2}, \varepsilon>0$, we have the following holds for all $x$ so that $\left|x-x_{1}\right| \wedge\left|x-x_{2}\right|>\left(\varepsilon_{1} \vee \varepsilon_{2} \vee \varepsilon\right)$ :

$$
\begin{cases}-U_{1,2}^{\vec{\lambda}, \vec{x}, \vec{\varepsilon}}(x) \leq \min \left\{2 \lambda_{2}^{-1}\left|x-x_{1}\right|^{-p} \varepsilon_{1}^{p-2},\right. & \left.2 \lambda_{1}^{-1}\left|x-x_{2}\right|^{-p} \varepsilon_{2}^{p-2}\right\}, \\ -V_{1,2}^{\vec{\lambda}, \vec{x}}(x) \leq \min \left\{2 \lambda_{2}^{-1} c_{3.2} \lambda_{1}^{-(1+\alpha)}\left|x-x_{1}\right|^{-p},\right. & \left.2 \lambda_{1}^{-1} c_{3.2} \lambda_{2}^{-(1+\alpha)}\left|x-x_{2}\right|^{-p}\right\}, \\ -W_{1,2}^{\vec{\lambda}, \vec{x}, \varepsilon}(x) \leq \min \left\{2 \lambda_{2}^{-1} c_{3.2} \lambda_{1}^{-(1+\alpha)}\left|x-x_{1}\right|^{-p},\right. & \left.2 \lambda_{1}^{-1}\left|x-x_{2}\right|^{-p} \varepsilon^{p-2}\right\} .\end{cases}
$$

Proof. Similar to the derivation of Lemma S.1.2 in [10], it is easy to conclude from the definition (see (4.8)) that $-U_{1,2}^{\vec{\lambda}, \vec{x}, \vec{\varepsilon}}(x)$ is strictly decreasing in $\vec{\lambda} \in(0, \infty)^{2}$. So we can use this monotonicity and $U_{2}^{\vec{\lambda}, \vec{x}, \vec{\varepsilon}} \geq 0$ (see (4.7)) to get

$$
-U_{1,2}^{\vec{\lambda}, \vec{x}, \vec{\varepsilon}}(x) \leq \frac{2}{\lambda_{1}} \int_{\lambda_{1} / 2}^{\lambda_{1}}-\frac{\partial}{\partial \lambda_{1}^{\prime}} U_{2}^{\left(\lambda_{1}^{\prime}, \lambda_{2}\right), \vec{x}, \vec{\varepsilon}}(x) d \lambda_{1}^{\prime} \leq \frac{2}{\lambda_{1}} U_{2}^{\left(\lambda_{1} / 2, \lambda_{2}\right), \vec{x}, \vec{\varepsilon}}(x) \leq \frac{2}{\lambda_{1}}\left|x-x_{2}\right|^{-p} \varepsilon_{2}^{p-2},
$$

where the last inequality is by (5.3). The result for $-U_{1,2}^{\vec{\lambda}, \vec{x}, \vec{\varepsilon}}$ follows by symmetry. The proofs for $-V_{1,2}^{\vec{\lambda}, \vec{x}}$ and $-W_{1,2}^{\vec{\lambda}, \vec{x}, \varepsilon}$ will follow in a similar way by using (5.2), (5.4) and (5.5).

Fix $x_{1} \neq x_{2}$ and $x \neq x_{1}, x_{2}$. Let $P_{x}$ denote the law of $d$-dimensional Brownian motion $B$ starting from $x$. Recall $r_{\lambda_{1}}, r_{\lambda_{2}}$ and $T_{r_{\lambda}}$ as in Lemma 8.2. The following result is from Lemma 9.5 of [19].

Lemma 9.2. For all $\lambda_{1}, \lambda_{2}>0$ large,

$$
\begin{aligned}
-V_{1,2}^{\vec{\lambda}, \vec{x}}(x)= & E_{x}\left(\int_{0}^{T_{r_{\lambda}}} \prod_{i=1}^{2} V_{i}^{\vec{\lambda}, \vec{x}}\left(B_{t}\right) \exp \left(-\int_{0}^{t} V^{\vec{\lambda}, \vec{x}}\left(B_{s}\right) d s\right) d t\right) \\
& +E_{x}\left(\exp \left(-\int_{0}^{T_{r_{\lambda}}} V^{\vec{\lambda}, \vec{x}}\left(B_{s}\right) d s\right) 1\left(T_{r_{\lambda}}<\infty\right)\left(-V_{1,2}^{\vec{\lambda}, \vec{x}}\left(B_{T_{r_{\lambda}}}\right)\right)\right) .
\end{aligned}
$$

Lemma 9.3. For all $\varepsilon_{1}, \varepsilon_{2}>0$ small, we have

$$
\begin{aligned}
-U_{1,2}^{\vec{\lambda}, \vec{x}, \vec{\varepsilon}}(x)= & E_{x}\left(\int_{0}^{T_{\varepsilon}} \prod_{i=1}^{2} U_{i}^{\vec{\lambda}, \vec{x}, \vec{\varepsilon}}\left(B_{t}\right) \exp \left(-\int_{0}^{t} U^{\vec{\lambda}, \vec{x}, \vec{\varepsilon}}\left(B_{s}\right) d s\right) d t\right) \\
& +E_{x}\left(\exp \left(-\int_{0}^{T_{\varepsilon}} U^{\vec{\lambda}, \vec{x}, \vec{\varepsilon}}\left(B_{s}\right) d s\right) 1\left(T_{\varepsilon}<\infty\right)\left(-U_{1,2}^{\vec{\lambda}, \vec{x}, \vec{\varepsilon}}\left(B_{T_{\varepsilon}}\right)\right)\right),
\end{aligned}
$$

where $T_{\varepsilon}$ is as in Lemma 8.4.

Proof. In view of Lemma 8.4, it follows in a similar manner to the proof of Lemma 9.2.

Lemma 9.4. For all $\lambda_{1}>0$ large and $\varepsilon>0$ small,

$$
\begin{aligned}
& -W_{1,2}^{\vec{\lambda}, \vec{x}, \varepsilon}(x)=E_{x}\left(\int_{0}^{T_{\lambda_{1}, \varepsilon}} \prod_{i=1}^{2} W_{i}^{\vec{\lambda}, \vec{x}, \vec{\varepsilon}}\left(B_{t}\right) \exp \left(-\int_{0}^{t} W^{\vec{\lambda}, \vec{x}, \vec{\varepsilon}}\left(B_{s}\right) d s\right) d t\right) \\
& +E_{x}\left(\exp \left(-\int_{0}^{T_{\lambda_{1}, \varepsilon}} W^{\vec{\lambda}, \vec{x}, \vec{\varepsilon}}\left(B_{s}\right) d s\right) 1\left(T_{\lambda_{1}, \varepsilon}<\infty\right)\left(-W_{1,2}^{\vec{\lambda}, \vec{x}, \vec{\varepsilon}}\left(B_{T_{\lambda_{1}, \varepsilon}}\right)\right)\right),
\end{aligned}
$$

where $T_{\lambda_{1}, \varepsilon}$ is as in Lemma 8.6.

Proof. In view of Lemma 8.6, it follows in a similar manner to the proof of Lemma 9.2. 
Lemma 9.5. For any $x_{1} \neq x_{2}$, if $\left|x-x_{1}\right| \wedge\left|x-x_{2}\right|>\varepsilon_{0}$ for some $\varepsilon_{0}>0$, then there is some constant $C_{9.5}\left(\varepsilon_{0}\right)>0$ so that for all $\varepsilon_{1}, \varepsilon_{2}>0$ small,

$$
\left.0 \leq \frac{1}{\varepsilon_{1}^{p-2}} \frac{1}{\varepsilon_{2}^{p-2}}\left(-U_{1,2}^{\vec{\lambda}, \vec{x}, \vec{\varepsilon}}(x)\right)\right) \leq C_{9.5}\left(\varepsilon_{0}\right)\left(1+\left|x_{1}-x_{2}\right|^{2-p}\right),
$$

and for all $\lambda_{1} \geq 1$ large and $\varepsilon>0$ small,

$$
\left.0 \leq \frac{\lambda_{1}^{1+\alpha}}{\varepsilon^{p-2}}\left(-W_{1,2}^{\vec{\lambda}, \vec{x}, \varepsilon}(x)\right)\right) \leq C_{9.5}\left(\varepsilon_{0}\right)\left(1+\left|x_{1}-x_{2}\right|^{2-p}\right) .
$$

Proof. In view of Lemma 9.3 and Lemma 9.4, it follows in a similar manner to the proof of Proposition 6.1 of [19] and Proposition 5.1 of [9].

\subsection{Proofs of Proposition 4.2 and 4.3(ii)}

Given the similarities of the proofs of Propositions 4.2(i), 4.2(ii) and 4.3(ii), we will only give the proof of 4.2(ii) here and other proofs can be found in Appendix D.

Proof of Proposition 4.2(ii). For any $x_{1} \neq x_{2}$, we fix any $x \neq x_{1}, x_{2}$. In order to find the limit of $\varepsilon_{1}^{-(p-2)} \varepsilon_{2}^{-(p-2)}\left(-U_{1,2}^{\vec{\lambda}, \vec{x}, \vec{\varepsilon}}(x)\right)$ as $\varepsilon_{1}, \varepsilon_{2} \downarrow 0$, by Lemma 9.3, it suffices to calculate the limits of following as $\varepsilon_{1}, \varepsilon_{2} \downarrow 0$.

$$
\begin{aligned}
K_{1}+K_{2} & :=\frac{1}{\varepsilon_{1}^{p-2}} \frac{1}{\varepsilon_{2}^{p-2}} E_{x}\left(\int_{0}^{T_{\varepsilon}} \prod_{i=1}^{2} U_{i}^{\vec{\lambda}, \vec{x}, \vec{\varepsilon}}\left(B_{t}\right) \exp \left(-\int_{0}^{t} U^{\vec{\lambda}, \vec{x}, \vec{\varepsilon}}\left(B_{s}\right) d s\right) d t\right) \\
& +\frac{1}{\varepsilon_{1}^{p-2}} \frac{1}{\varepsilon_{2}^{p-2}} E_{x}\left(\exp \left(-\int_{0}^{T_{\varepsilon}} U^{\vec{\lambda}, \vec{x}, \vec{\varepsilon}}\left(B_{s}\right) d s\right) 1_{\left(T_{\varepsilon}<\infty\right)}\left(-U_{1,2}^{\vec{\lambda}, \vec{x}, \vec{\varepsilon}}\left(B_{T_{\varepsilon}}\right)\right)\right) .
\end{aligned}
$$

Recall $T_{\varepsilon}=T_{2 \varepsilon_{i}}^{i} \wedge T_{2 \varepsilon_{3-i}}^{3-i}$ where $T_{2 \varepsilon_{i}}^{i}=\inf \left\{t \geq 0:\left|B_{t}\right| \leq 2 \varepsilon_{i}\right\}, i=1,2$. Let $\varepsilon_{1}, \varepsilon_{2}>0$ be small so that $0<4\left(\varepsilon_{1} \vee \varepsilon_{2}\right)<\left|x_{1}-x_{2}\right|$.

We first consider $K_{2}$. On $\left\{T_{\varepsilon}<\infty\right\}$, by considering $T_{\varepsilon}=T_{2 \varepsilon_{i}}^{i}<T_{2 \varepsilon_{3-i}}^{3-i}$ we may set $B_{T_{\varepsilon}}=B_{T_{2 \varepsilon_{i}}^{i}}$ so that $\left|B_{T_{2 \varepsilon_{i}}^{i}}-x_{i}\right|=2 \varepsilon_{i}$ and $\left|x_{3-i}-B_{T_{2 \varepsilon_{i}}^{i}}\right| \geq \Delta / 2$ where $\Delta=\left|x_{1}-x_{2}\right|$. Lemma 9.1 and the above imply

$$
-U_{1,2}^{\vec{\lambda}, \vec{x}, \vec{\varepsilon}}\left(B_{T_{\varepsilon}}\right)=-U_{1,2}^{\vec{\lambda}, \vec{x}, \vec{\varepsilon}}\left(B_{T_{2 \varepsilon_{i}}^{i}}\right) \leq \frac{2}{\lambda_{i}} \Delta^{-p} 2^{p} \varepsilon_{3-i}^{p-2} \leq c \Delta^{-p} \varepsilon_{3-i}^{p-2}
$$

This shows that

$$
K_{2} \leq \frac{1}{\varepsilon_{1}^{p-2}} \frac{1}{\varepsilon_{2}^{p-2}} \sum_{i=1}^{2} c \Delta^{-p} \varepsilon_{3-i}^{p-2} E_{x}\left(1_{\left\{T_{2 \varepsilon_{i}}^{i}<\infty\right\}} 1_{\left\{T_{2 \varepsilon_{i}}^{i}<T_{2 \varepsilon_{3-i}}^{3-i}\right\}} \exp \left(-\int_{0}^{T_{2 \varepsilon_{i}}^{i}} U^{\vec{\lambda}, \vec{x}, \vec{\varepsilon}}\left(B_{s}\right) d s\right)\right) .
$$

From (8.14) we have for $i=1,2$,

$$
E_{x}\left(1_{\left\{T_{2 \varepsilon_{i}}^{i}<\infty\right\}} 1_{\left\{T_{2 \varepsilon_{i}}^{i}<T_{2 \varepsilon_{3-i}}^{3-i}\right\}} \exp \left(-\int_{0}^{T_{2 \varepsilon_{i}}^{i}} U^{\vec{\lambda}, \vec{x}, \vec{\varepsilon}}\left(B_{s}\right) d s\right)\right) \leq\left(2 \varepsilon_{i}\right)^{p}\left|x-x_{i}\right|^{-p},
$$

and so (9.4) becomes

$$
\begin{aligned}
K_{2} & \leq \frac{1}{\varepsilon_{1}^{p-2}} \frac{1}{\varepsilon_{2}^{p-2}} c \Delta^{-p} \sum_{i=1}^{2} \varepsilon_{3-i}^{p-2}\left(2 \varepsilon_{i}\right)^{p}\left|x-x_{i}\right|^{-p} \\
& \leq C \Delta^{-p}\left(\varepsilon_{1}^{2}+\varepsilon_{2}^{2}\right) \sum_{i=1}^{2}\left|x-x_{i}\right|^{-p} \rightarrow 0 \text { as } \varepsilon_{1}, \varepsilon_{2} \downarrow 0 .
\end{aligned}
$$


Turning to $K_{1}$, we first recall

$$
K_{1}=\iint_{0}^{\infty} \frac{1}{\varepsilon_{1}^{p-2}} \frac{1}{\varepsilon_{2}^{p-2}} U_{1}^{\vec{\lambda}, \vec{x}, \vec{\varepsilon}}\left(B_{t}\right) U_{2}^{\vec{\lambda}, \vec{x}, \vec{\varepsilon}}\left(B_{t}\right) \exp \left(-\int_{0}^{t} U^{\vec{\lambda}, \vec{x}, \vec{\varepsilon}}\left(B_{s}\right) d s\right) 1_{\left(t \leq T_{\varepsilon}\right)} d t d P_{x} .
$$

We know $T_{\varepsilon} \rightarrow \infty$ as $\varepsilon_{1}, \varepsilon_{2} \downarrow 0$ since Brownian motion in $d \geq 2$ doesn't hit points. By Proposition 4.1 and Lemma 8.1, for $L e b \times P_{x}$-a.e. $(t, \omega)$, we have

$$
\begin{aligned}
\lim _{\varepsilon_{1}, \varepsilon_{2} \downarrow 0} & \frac{1}{\varepsilon_{1}^{p-2}} \frac{1}{\varepsilon_{2}^{p-2}} U_{1}^{\vec{\lambda}, \vec{x}, \vec{\varepsilon}}\left(B_{t}\right) U_{2}^{\vec{\lambda}, \vec{x}, \vec{\varepsilon}}\left(B_{t}\right) \exp \left(-\int_{0}^{t} U^{\vec{\lambda}, \vec{x}, \vec{\varepsilon}}\left(B_{s}\right) d s\right) 1\left(t \leq T_{\varepsilon}\right) \\
= & C_{4.1}\left(\lambda_{1}\right) C_{4.1}\left(\lambda_{2}\right) U_{1}^{\vec{\infty}, \vec{x}}\left(B_{t}\right) U_{2}^{\vec{\infty}, \vec{x}}\left(B_{t}\right) \exp \left(-\int_{0}^{t} V^{\vec{\infty}, \vec{x}}\left(B_{s}\right) d s\right) .
\end{aligned}
$$

Recall the definition of $U_{1,2}^{\vec{\infty}, \vec{x}}(x)$ as in (1.17). If we can find an integrable bound for the left-hand side term of (9.7), by the dominated convergence theorem we can conclude from (9.6) and (9.7) that

$$
\lim _{\varepsilon_{1}, \varepsilon_{2} \downarrow 0} K_{1}=C_{4.1}\left(\lambda_{1}\right) C_{4.1}\left(\lambda_{2}\right)\left(-U_{1,2}^{\vec{\infty}, \vec{x}}(x)\right),
$$

and the proof will be finished by Lemma 9.3, (9.3), (9.5) and (9.8).

It suffices to find an integrable bound for the left-hand side term of (9.7). Recall (5.3) and (8.7) to see that

$$
\begin{aligned}
& \frac{1}{\varepsilon_{1}^{p-2}} \frac{1}{\varepsilon_{2}^{p-2}} U_{1}^{\vec{\lambda}, \vec{x}, \vec{\varepsilon}}\left(B_{t}\right) U_{2}^{\vec{\lambda}, \vec{x}, \vec{\varepsilon}}\left(B_{t}\right) \exp \left(-\int_{0}^{t} U^{\vec{\lambda}, \vec{x}, \vec{\varepsilon}}\left(B_{s}\right) d s\right) 1\left(t \leq T_{\varepsilon}\right) \\
\leq & \left|B_{t}-x_{1}\right|^{-p}\left|B_{t}-x_{2}\right|^{-p} \exp \left(-\int_{0}^{t} U^{\vec{\lambda}, \vec{x}, \vec{\varepsilon}}\left(B_{s}\right) d s\right) 1\left(t \leq T_{\varepsilon}\right) \\
\leq & \sum_{i=1}^{2}\left|B_{t}-x_{i}\right|^{-p}\left|B_{t}-x_{3-i}\right|^{-p} 1\left(\left|B_{t}-x_{i}\right| \leq\left|B_{t}-x_{3-i}\right|\right) \exp \left(-\int_{0}^{t} V^{\infty}\left(B_{s}-x_{i}\right) d s\right) \\
\leq & 2^{p} \sum_{i=1}^{2}\left|B_{t}-x_{i}\right|^{-p}\left(\left|B_{t}-x_{i}\right|^{-p} \wedge \Delta^{-p}\right) \exp \left(-\int_{0}^{t} V^{\infty}\left(B_{s}-x_{i}\right) d s\right),
\end{aligned}
$$

where in the last inequality we have used $\left|B_{t}-x_{3-i}\right| \geq\left(\left|B_{t}-x_{i}\right| \vee(\Delta / 2)\right)$ on the set $\left\{\left|B_{t}-x_{i}\right| \leq\left|B_{t}-x_{3-i}\right|\right\}$.

It remains to show that for $i=1,2$,

$$
\begin{aligned}
I_{i}:=\iint_{0}^{\infty}\left|B_{t}-x_{i}\right|^{-p}\left(\left|B_{t}-x_{i}\right|^{-p} \wedge \Delta^{-p}\right) \\
\exp \left(-\int_{0}^{t} V^{\infty}\left(B_{s}-x_{i}\right) d s\right) d t d P_{x}<\infty .
\end{aligned}
$$

Let $r_{\varepsilon}=2 \varepsilon$. For $i=1,2$, by translation invariance and the monotone convergence theorem we have

$$
\begin{aligned}
I_{i} & =E_{x-x_{i}}\left(\int_{0}^{\infty}\left|B_{t}\right|^{-p}\left(\left|B_{t}\right|^{-p} \wedge \Delta^{-p}\right) \exp \left(-\int_{0}^{t} V^{\infty}\left(B_{s}\right) d s\right) d t\right) \\
& =\lim _{\varepsilon \downarrow 0} E_{x-x_{i}}\left(\int_{0}^{\tau_{r_{\varepsilon}}}\left|B_{t}\right|^{-p}\left(\left|B_{t}\right|^{-p} \wedge \Delta^{-p}\right) \exp \left(-\int_{0}^{t} V^{\infty}\left(B_{s}\right) d s\right) d t\right) .
\end{aligned}
$$

By (S.18) and (S.20) of [10], we have

$$
\begin{aligned}
E_{x-x_{i}}\left(\int_{0}^{\tau_{r_{\varepsilon}}}\left|B_{t}\right|^{-p}\left(\left|B_{t}\right|^{-p} \wedge \Delta^{-p}\right) \exp \left(\int_{0}^{t} \frac{2^{p} D^{\lambda}(2) \varepsilon^{p-2}}{\left|B_{s}\right|^{p}} d s\right)\right. \\
\left.\quad \exp \left(-\int_{0}^{t} \frac{2(4-d)}{\left|B_{s}\right|^{2}} d s\right) d t\right) \leq C \Delta^{2-p}\left|x-x_{i}\right|^{-p}, \forall \varepsilon>0 \text { small, }
\end{aligned}
$$


where $D^{\lambda}(2)=U^{\infty, 1}(2)-U^{\lambda, 1}(2)$ with $\lambda>0$ large. Therefore we conclude from (9.11) and (9.12) that $I_{i}<\infty$ and the proof of (9.10) is complete. It remains to prove (4.13). Recall the definition of $\left(-U_{1,2}^{\vec{\infty}, \vec{x}}(x)\right)$ as in (1.17). By (1.16), (8.32) and (9.9), it follows immediately from (9.11) and (9.12).

\section{A Proofs of Theorems 1.4 and 1.14 under $\mathbb{P}_{X_{0}}$}

We deal with the case $\mathbb{P}_{X_{0}}$ for the general initial condition $X_{0}$ and recall $S\left(X_{0}\right)$ is the closed support of $X_{0}$. Recall $S\left(X_{0}\right)^{\geq \delta}=\left\{x: d\left(x, S\left(X_{0}\right)\right) \geq \delta\right\}$ for any $\delta>0$, where $d\left(x, S\left(X_{0}\right)\right)=\inf \left\{|x-y|: y \in S\left(X_{0}\right)\right\}$. Similarly we define $S\left(X_{0}\right)^{>\delta}, S\left(X_{0}\right) \leq \delta$ and $S\left(X_{0}\right)<\delta$.

We first give the convergence of $\mathcal{L}^{\lambda}$ to $\mathcal{L}$ and $\widetilde{\mathcal{L}}(\kappa)^{\varepsilon}$ to $\widetilde{\mathcal{L}}(\kappa)$ and then find some constant $c_{1.13}(\kappa)>0$ so that $\widetilde{\mathcal{L}}(\kappa)=c_{1.13}(\kappa) \mathcal{L}$ a.s. Next we show that the support of $\widetilde{\mathcal{L}}(\kappa)$ is contained in $\partial \mathcal{R} \cap S\left(X_{0}\right)^{c}$ and it follows that the support of $\mathcal{L}$ will also be on $\partial \mathcal{R} \cap S\left(X_{0}\right)^{c}$, thus finishing both proofs of Theorem 1.4 and Theorem 1.14. Since the proof for the convergence of $\mathcal{L}^{\lambda}$ and $\widetilde{\mathcal{L}}(\kappa)^{\varepsilon}$ are similar, we will only give the proof for the latter.

Let $\left\{\phi_{m}\right\}_{m=1}^{\infty}$ be a countable determining class for $M_{F}\left(\mathbb{R}^{d}\right)$ consisting of bounded, continuous functions and we take $\phi_{1}=1$. Define

$$
\mathcal{C}_{X_{0}}=\left\{\psi_{m, k}^{X_{0}}: \psi_{m, k}^{X_{0}}=\phi_{m} \cdot \chi_{k}^{X_{0}}, m \geq 1, k \geq 1\right\}
$$

where

$$
\chi_{k}^{X_{0}}(x)= \begin{cases}1, & \text { if } x \in B_{k} \cap S\left(X_{0}\right)^{>1 / k} \\ 0, & \text { if } d\left(x, S\left(X_{0}\right)\right) \leq(2 k)^{-1} \text { or }|x| \geq k+1 \\ \text { continuous, }, & \text { on }(2 k)^{-1} \leq d\left(x, S\left(X_{0}\right)\right) \leq k^{-1} \text { and } k \leq|x| \leq k+1 .\end{cases}
$$

Corollary 5.5 implies that for any $\psi_{m, k}^{X_{0}} \in \mathcal{C}_{X_{0}}$, we have $\widetilde{\mathcal{L}}(\kappa)^{\varepsilon}\left(\psi_{m, k}^{X_{0}}\right)$ converges in $L^{2}\left(\mathbb{P}_{X_{0}}\right)$ to some $\widetilde{l}\left(\psi_{m, k}^{X_{0}}\right)$ and by taking a subsequence we get almost sure convergence. Define subsequences iteratively and take a diagonal subsequence $\varepsilon_{n} \downarrow 0$ (we may assume $0<\varepsilon_{n}<1$ for all $n \geq 1$ ) to get

$$
\widetilde{\mathcal{L}}(\kappa)^{\varepsilon_{n}}\left(\psi_{m, k}^{X_{0}}\right) \rightarrow \widetilde{l}\left(\psi_{m, k}^{X_{0}}\right) \text { as } \varepsilon_{n} \downarrow 0 \text {, for all } m, k \geq 1, \mathbb{P}_{X_{0}} \text {-a.s. }
$$

For any fixed $0<\delta<1$ we will consider the restriction of $\left\{\widetilde{\mathcal{L}}(\kappa)^{\varepsilon_{n}}\right\}$ to $S\left(X_{0}\right)^{\geq \delta}$ and we write $\left.\widetilde{\mathcal{L}}(\kappa)_{\delta}^{\varepsilon_{n}} \equiv \widetilde{\mathcal{L}}(\kappa)^{\varepsilon_{n}}\right|_{S\left(X_{0}\right) \geq \delta}\left(\right.$ recall $\left.\mu\right|_{K}(\cdot)=\mu(\cdot \cap K)$ ).

First we use Corollary III.1.5 of [20] to see that with $\mathbb{P}_{X_{0}}$-probability one there is some $\beta^{\prime}(\omega) \in(0,1]$ such that for all $0<t<\beta^{\prime}$, the closed support of $X_{t}$ is within the region $\left\{x: d\left(x, S\left(X_{0}\right)\right)<3(t \log (1 / t))^{1 / 2}\right\}$. Pick $0<\beta<\beta^{\prime}$ small enough so that $3(\beta \log (1 / \beta))^{1 / 2}<\delta$ and hence

$$
\mathcal{R} \cap S\left(X_{0}\right)^{\geq \delta} \subset \bigcup_{t \geq \beta} \operatorname{Supp}\left(X_{t}\right) .
$$

By Corollary III.1.7 of [20] we conclude from the above that for any $\delta>0$,

$$
\mathcal{R} \cap S\left(X_{0}\right)^{\geq \delta} \text { is bounded, } \quad \mathbb{P}_{X_{0}} \text {-a.s. }
$$

Next we claim that for any $0<\delta<1$ and any $\varepsilon_{n} \downarrow 0$, with $\mathbb{P}_{X_{0}}$-probability one we have

$$
\sup _{0<\varepsilon_{n}<\delta / 2} \widetilde{\mathcal{L}}(\kappa)^{\varepsilon_{n}}\left(\left(\mathcal{R} \cap S\left(X_{0}\right)^{\geq \delta / 2}\right)^{>1} \cap S\left(X_{0}\right)^{\geq \delta}\right)=0
$$


To see this, we fix $\varepsilon<\delta / 2<1$. For all $x \in\left(\mathcal{R} \cap S\left(X_{0}\right)^{\geq \delta / 2}\right)^{>1}$, we have

$$
\overline{B_{\varepsilon}(x)} \subset\left(\mathcal{R} \cap S\left(X_{0}\right)^{\geq \delta / 2}\right)^{c} .
$$

Next for all $x \in S\left(X_{0}\right)^{\geq \delta}$, we have $\overline{B_{\varepsilon}(x)} \subset S\left(X_{0}\right)^{>\delta / 2}$ and in particular

$$
\overline{B_{\varepsilon}(x)} \subset\left(\mathcal{R} \cap S\left(X_{0}\right)^{\leq \delta / 2}\right)^{c} .
$$

Therefore we conclude $x \in\left(\mathcal{R} \cap S\left(X_{0}\right)^{\geq \delta / 2}\right)^{>1} \cap S\left(X_{0}\right)^{\geq \delta}$ would imply $\overline{B_{\varepsilon}(x)} \subset \mathcal{R}^{c}$, and by (2.4) we have $X_{G_{\varepsilon}^{x}}(1)=0$. It follows that for all $0<\varepsilon_{n}<\delta / 2$,

$$
\begin{aligned}
& \mathbb{E}_{X_{0}}\left(\widetilde{\mathcal{L}}(\kappa)^{\varepsilon_{n}}\left(\left(\mathcal{R} \cap S\left(X_{0}\right)^{\geq \delta / 2}\right)^{>1} \cap S\left(X_{0}\right)^{\geq \delta}\right)\right) \\
& \leq \mathbb{E}_{X_{0}}\left(\int \frac{X_{G_{\varepsilon}^{x}}(1)}{\varepsilon^{p}} \exp \left(-\kappa \frac{X_{G_{\varepsilon}^{x}}(1)}{\varepsilon^{2}}\right) 1\left(\overline{B_{\varepsilon}(x)} \subset \mathcal{R}^{c}\right) d x\right) \\
& =\int \mathbb{E}_{X_{0}}\left(\frac{X_{G_{\varepsilon}^{x}}(1)}{\varepsilon^{p}} \exp \left(-\kappa \frac{X_{G_{\varepsilon}^{x}}(1)}{\varepsilon^{2}}\right) 1\left(\overline{B_{\varepsilon}(x)} \subset \mathcal{R}^{c}\right)\right) d x=0 .
\end{aligned}
$$

Thus we get (A.4) by taking a countable union of null sets.

Now use (A.3) and (A.4) to see that with $\mathbb{P}_{X_{0}}$-probability one, for $M \geq 1$ large we have

$$
\begin{gathered}
\sup _{0<\varepsilon_{n}<\delta / 2} \widetilde{\mathcal{L}}(\kappa)_{\delta}^{\varepsilon_{n}}(\{x:|x| \geq M\}) \leq \sup _{0<\varepsilon_{n}<\delta / 2} \widetilde{\mathcal{L}}(\kappa)_{\delta}^{\varepsilon_{n}}\left(\left(\mathcal{R} \cap S\left(X_{0}\right)^{\geq \delta / 2}\right)^{>1}\right) \\
=\sup _{0<\varepsilon_{n}<\delta / 2} \widetilde{\mathcal{L}}(\kappa)^{\varepsilon_{n}}\left(\left(\mathcal{R} \cap S\left(X_{0}\right)^{\geq \delta / 2}\right)^{>1} \cap S\left(X_{0}\right)^{\geq \delta}\right)=0 .
\end{gathered}
$$

For any $M>1$, by using (A.2) with $m=1$, we conclude with $\mathbb{P}_{X_{0}}$-probability one, for $k \geq 1$ large, we have

$$
\sup _{0<\varepsilon_{n}<\delta / 2} \widetilde{\mathcal{L}}(\kappa)_{\delta}^{\varepsilon_{n}}\left(B_{M}\right)=\sup _{0<\varepsilon_{n}<\delta / 2} \widetilde{\mathcal{L}}(\kappa)^{\varepsilon_{n}}\left(S\left(X_{0}\right)^{\geq \delta} \cap B_{M}\right) \leq \sup _{0<\varepsilon_{n}<\delta / 2} \widetilde{\mathcal{L}}(\kappa)^{\varepsilon_{n}}\left(\chi_{k}^{X_{0}}\right)<\infty .
$$

Together with (A.5), we have

$$
\sup _{0<\varepsilon_{n}<\delta / 2} \widetilde{\mathcal{L}}(\kappa)_{\delta}^{\varepsilon_{n}}(1) \leq \sup _{0<\varepsilon_{n}<\delta / 2} \widetilde{\mathcal{L}}(\kappa)_{\delta}^{\varepsilon_{n}}\left(B_{M}\right)+\sup _{0<\varepsilon_{n}<\delta / 2} \widetilde{\mathcal{L}}(\kappa)_{\delta}^{\varepsilon_{n}}(\{x:|x| \geq M\})<\infty
$$

Note (A.5) also implies the tightness of $\left\{\widetilde{\mathcal{L}}(\kappa)_{\delta}^{\varepsilon_{n}}, 0<\varepsilon_{n}<\delta / 2\right\}$ and together with (A.6), we get the relative compactness of $\left\{\widetilde{\mathcal{L}}(\kappa)_{\delta}^{\varepsilon_{n}}, 0<\varepsilon_{n}<\delta / 2\right\}$ by Prohorov's theorem (see, e.g., Theorem 7.8.7 of [1]). Therefore any subsequence admits a further sequence along which the measures converge to some $\widetilde{l}(\kappa)_{\delta}$ supported on $S\left(X_{0}\right)^{\geq \delta}$ in the weak topology. It remains to check all limit point coincide which is easy to see by (A.2) since $\mathcal{C}_{X_{0}}$ is a determining class on $M_{F}\left(S\left(X_{0}\right)^{\geq \delta}\right)$. Therefore for any $\delta>0$, under $\mathbb{P}_{X_{0}}$ we have $\widetilde{\mathcal{L}}(\kappa)_{\delta}^{\varepsilon_{n}} \stackrel{P}{\rightarrow} \widetilde{l}(\kappa)_{\delta}$ as $\varepsilon \downarrow 0$.

Note by definition, $\widetilde{l}(\kappa)_{\delta^{\prime}}$ and $\widetilde{l}(\kappa)_{\delta}$ agree on $S\left(X_{0}\right)^{\geq \delta}$ for all $\delta \geq \delta^{\prime}>0$. Take $\delta=1 / k$ and define a $\sigma$-finite measure $\widetilde{\mathcal{L}}(\kappa)$ on $S\left(X_{0}\right)^{c}$ by

$$
\left.\widetilde{\mathcal{L}}(\kappa)\right|_{S\left(X_{0}\right) \geq 1 / k} \equiv \widetilde{l}(\kappa)_{1 / k}, \forall k \geq 1 .
$$

Thus we conclude $\left.\left.\widetilde{\mathcal{L}}(\kappa)^{\varepsilon}\right|_{S\left(X_{0}\right) \geq 1 / k} \stackrel{P}{\rightarrow} \widetilde{\mathcal{L}}(\kappa)\right|_{S\left(X_{0}\right) \geq 1 / k}$ as $\varepsilon \downarrow 0$ under $\mathbb{P}_{X_{0}}$ for all $k \geq 1$ and by taking a diagonal subsequence, we can find some sequence $\varepsilon_{n} \downarrow 0$ so that $\left.\left.\widetilde{\mathcal{L}}(\kappa)^{\varepsilon_{n}}\right|_{S\left(X_{0}\right) \geq 1 / k} \rightarrow \widetilde{\mathcal{L}}(\kappa)\right|_{S\left(X_{0}\right) \geq 1 / k}, \forall k \geq 1$ a.s. as $n \rightarrow \infty$.

With the construction of $\widetilde{\mathcal{L}}(\kappa)$, and by a similar argument for the construction of $\mathcal{L}$ complete under $\mathbb{P}_{X_{0}}$, we now show $\mathbb{P}_{X_{0}}$-a.s. that $\widetilde{\mathcal{L}}(\kappa)=c_{1.13}(\kappa) \mathcal{L}$. By the above 
construction, it suffices to show that for any $k \geq 1$, we have $\mathbb{P}_{X_{0}}$-a.s. that $\left.\widetilde{\mathcal{L}}(\kappa)\right|_{S\left(X_{0}\right) \geq 1 / k}=$ $\left.c_{1.13}(\kappa) \mathcal{L}\right|_{S\left(X_{0}\right) \geq 1 / k}$.

Similar to the derivation of (5.18), by Corollary 5.5 and Corollary 5.6, we can get $\mathbb{P}_{X_{0}}$-a.s. that $C_{4.1}(\kappa) \mathcal{L}\left(\psi_{m, k}^{X_{0}}\right)=K_{4.1} \widetilde{\mathcal{L}}(\kappa)\left(\psi_{m, k}^{X_{0}}\right)$ for all $m, k \geq 1$ and so we have

$$
\left.C_{4.1}(\kappa) \mathcal{L}\right|_{S\left(X_{0}\right) \geq 1 / k}=\left.K_{4.1} \widetilde{\mathcal{L}}(\kappa)\right|_{S\left(X_{0}\right) \geq 1 / k} \text { for all } k \geq 1 .
$$

Then it follows that $\mathbb{P}_{X_{0}}$-a.s. $\widetilde{\mathcal{L}}(\kappa)=c_{1.13}(\kappa) \mathcal{L}$ as noted above.

Finally by using Proposition 5.7, one can show that $\widetilde{\mathcal{L}}(\kappa)$ (and hence $\mathcal{L}$ ) is supported on $\partial \mathcal{R}$ in a similar way to the proof of Theorem 1.3 under $\mathbb{N}_{0}$ in Section 5.2. The construction of $\widetilde{\mathcal{L}}(\kappa)$ will then give us that $\widetilde{\mathcal{L}}(\kappa)$ is supported on $\partial \mathcal{R} \cap S\left(X_{0}\right)^{c}$. The proof is then complete.

\section{B Proofs of Lemmas 8.1 and 8.3}

\section{B.1 Proof of Lemma 8.1}

Recall from (1.15) that

$$
V^{\infty, \vec{x}}(x)=\mathbb{N}_{x}\left(\left\{L^{x_{1}}>0\right\} \cup\left\{L^{x_{2}}>0\right\}\right)<\infty,
$$

where the finiteness is by $\mathbb{N}_{0}\left(L^{x}>0\right)=V^{\infty}(x)<\infty$. Therefore by (4.3) and the monotone convergence theorem we have

$$
\begin{aligned}
& V^{\infty, \vec{x}}(x)-V^{\vec{\lambda}, \vec{x}}(x)=\mathbb{N}_{x}\left(1-1_{\left\{L^{x_{1}}=0\right\} \cap\left\{L^{x_{2}}=0\right\}}\right)-\mathbb{N}_{x}\left(1-e^{-\lambda_{1} L^{x_{1}}-\lambda_{2} L^{x_{2}}}\right) \\
& =\mathbb{N}_{x}\left(e^{-\lambda_{1} L^{x_{1}}-\lambda_{2} L^{x_{2}}} 1_{\left\{L^{x_{1}}>0\right\} \cup\left\{L^{x_{2}}>0\right\}}\right) \rightarrow 0 \text { as } \lambda_{1}, \lambda_{2} \rightarrow \infty .
\end{aligned}
$$

Now we will turn to the proof for $U^{\vec{\lambda}, \vec{x}, \vec{\varepsilon}}$ and $W^{\vec{\lambda}, \vec{x}, \varepsilon}$. For any $x$ so that $\left|x-x_{i}\right|>\varepsilon_{i}, i=1,2$, we first define $K_{1}, K_{2}$ by

$$
\begin{aligned}
K_{i} \equiv & \mathbb{N}_{x}\left(\left(\exp \left(-\lambda_{i} \frac{X_{G_{\varepsilon_{i}}^{x_{i}}}(1)}{\varepsilon_{i}^{2}}\right) 1\left(X_{G_{\varepsilon_{i} / 2}^{x_{i}}}=0\right)-1_{\left\{L^{x_{i}}=0\right\}}\right)^{2}\right) \\
= & \mathbb{N}_{x}\left(\left(\exp \left(-2 \lambda_{i} \frac{X_{G_{\varepsilon_{i}}^{x_{i}}(1)}}{\varepsilon_{i}^{2}}\right) 1\left(X_{G_{\varepsilon_{i} / 2}^{x_{i}}}=0\right)\right.\right. \\
& \left.\quad-2 \exp \left(-\lambda_{i} \frac{X_{G_{\varepsilon_{i}}^{x_{i}}(1)}}{\varepsilon_{i}^{2}}\right) 1\left(X_{G_{\varepsilon_{i} / 2}^{x_{i}}}=0\right) 1_{\left\{L^{x_{i}}=0\right\}}+1_{\left\{L^{x_{i}}=0\right\}}\right) .
\end{aligned}
$$

Use Proposition 2.1(i) to see that

$$
\begin{aligned}
K_{i}= & \mathbb{N}_{x}\left(\left(\exp \left(-2 \lambda_{i} \frac{X_{G_{i}^{x_{i}}}(1)}{\varepsilon_{i}^{2}}\right) \mathbb{P}_{X_{G_{\varepsilon_{i}}^{x_{i}}}}\left(X_{G_{\varepsilon_{i} / 2}^{x_{i}}}=0\right)\right.\right. \\
& \left.-2 \exp \left(-\lambda_{i} \frac{X_{G_{i}^{x_{i}}}(1)}{\varepsilon_{i}^{2}}\right) \mathbb{P}_{X_{G_{\varepsilon_{i}}^{x_{i}}}}\left(X_{G_{\varepsilon_{i} / 2}^{x_{i}}}=0, L^{x_{i}}=0\right)+\mathbb{P}_{X_{G_{\varepsilon_{i}}^{x_{i}}}}\left(L^{x_{i}}=0\right)\right) .
\end{aligned}
$$

Apply (1.27) to see that

$$
\mathbb{P}_{X_{G_{\varepsilon_{i}}^{x_{i}}}}\left(L^{x_{i}}=0\right)=\exp \left(-V^{\infty}\left(\varepsilon_{i}\right) X_{G_{\varepsilon_{i}}^{x_{i}}}(1)\right)=\exp \left(-\lambda_{d} \frac{X_{G_{\varepsilon_{i}}^{x_{i}}}(1)}{\varepsilon_{i}^{2}}\right),
$$

and as in the derivation of (3.20), we have

$$
\mathbb{P}_{X_{\varepsilon_{\varepsilon}}^{x_{i}}}\left(X_{G_{\varepsilon_{i} / 2}^{x_{i}}}=0\right)=\exp \left(-4 U^{\infty, 1}(2) \frac{X_{G_{i}^{x_{i}}}(1)}{\varepsilon_{i}^{2}}\right) .
$$


Use Proposition 2.2(i) to get

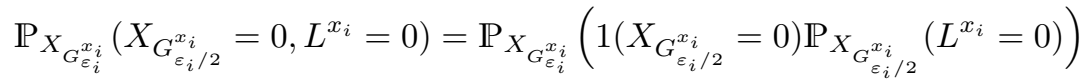

$$
\begin{aligned}
& =\mathbb{P}_{X_{G_{i}}^{x_{i}}}\left(X_{G_{\varepsilon_{i} / 2}^{x_{i}}}=0\right) \text {. }
\end{aligned}
$$

Returning to (B.2), we have

$$
\begin{aligned}
K_{i}= & \mathbb{N}_{x}\left(\exp \left(-\left(2 \lambda_{i}+4 U^{\infty, 1}(2)\right) \frac{X_{G_{\varepsilon_{i}}^{x_{i}}}(1)}{\varepsilon_{i}^{2}}\right)\right. \\
& \left.\quad-2 \exp \left(-\left(\lambda_{i}+4 U^{\infty, 1}(2)\right) \frac{X_{G_{\varepsilon_{i}}^{x_{i}}}(1)}{\varepsilon_{i}^{2}}\right)+\exp \left(-\lambda_{d} \frac{X_{G_{\varepsilon_{i}}^{x_{i}}}(1)}{\varepsilon_{i}^{2}}\right)\right) \\
\leq & \mathbb{N}_{x}\left(\exp \left(-\lambda_{d} \frac{X_{G_{\varepsilon_{i}}^{x_{i}}}(1)}{\varepsilon_{i}^{2}}\right)-\exp \left(-\left(\lambda_{i}+4 U^{\infty, 1}(2)\right) \frac{X_{G_{\varepsilon_{i}}^{x_{i}}(1)}}{\varepsilon_{i}^{2}}\right)\right) \\
= & U^{\left(\lambda_{i}+4 U^{\infty, 1}(2)\right) \varepsilon_{i}^{-2}, \varepsilon_{i}}\left(x-x_{i}\right)-U^{\lambda_{d} \varepsilon_{i}^{-2}, \varepsilon_{i}}\left(x-x_{i}\right) \rightarrow 0 \text { as } \varepsilon_{i} \downarrow 0,
\end{aligned}
$$

where the second equality is by (3.18) and the convergence to 0 follows from (8.29).

Turning to $U^{\vec{\lambda}, \vec{x}, \vec{\varepsilon}}(x)$, for $\varepsilon_{1}, \varepsilon_{2}>0$ small enough, by definition we have

$$
\begin{aligned}
I & =U^{\vec{\lambda}, \vec{x}, \vec{\varepsilon}}(x)-V^{\infty, \vec{x}}(x) \\
& =\mathbb{N}_{x}\left(1-\prod_{i=1}^{2} \exp \left(-\lambda_{i} \frac{X_{G_{i}^{x_{i}}}(1)}{\varepsilon_{i}^{2}}\right) 1\left(X_{G_{\varepsilon_{i} / 2}^{x_{i}}}=0\right)\right)-\mathbb{N}_{x}\left(1-1_{\left\{L^{x_{1}}=0\right\}} 1_{\left\{L^{x_{2}}=0\right\}}\right) \\
& =\mathbb{N}_{x}\left(1_{\left\{L^{x_{1}}=0\right\}} 1_{\left\{L^{x_{2}}=0\right\}}-\prod_{i=1}^{2} \exp \left(-\lambda_{i} \frac{X_{G_{\varepsilon_{i}}^{x_{i}}}(1)}{\varepsilon_{i}^{2}}\right) 1\left(X_{G_{\varepsilon_{i} / 2}^{x_{i}}}=0\right)\right) .
\end{aligned}
$$

By Jensen's inequality we have

$$
\begin{aligned}
& I^{2} \leq \mathbb{N}_{x}\left(\left(\prod_{i=1}^{2} \exp \left(-\lambda_{i} \frac{X_{G_{i}^{x_{i}}}(1)}{\varepsilon_{i}^{2}}\right) 1\left(X_{G_{\varepsilon_{i} / 2}^{x_{i}}}=0\right)-1_{\left\{L^{x_{1}}=0\right\}} 1_{\left\{L^{x_{2}}=0\right\}}\right)^{2}\right) \\
& \leq 2 \mathbb{N}_{x}\left(\left(\prod_{i=1}^{2} \exp \left(-\lambda_{i} \frac{X_{G_{i}^{x_{i}}}(1)}{\varepsilon_{i}^{2}}\right) 1\left(X_{G_{\varepsilon_{i} / 2}^{x_{i}}}=0\right)\right.\right. \\
& \left.\left.\quad-1_{\left\{L^{x_{1}}=0\right\}} \exp \left(-\lambda_{2} \frac{X_{G_{\varepsilon_{2}}^{x_{2}}(1)}}{\varepsilon_{2}^{2}}\right) 1\left(X_{G_{\varepsilon_{2} / 2}^{x_{2}}}=0\right)\right)^{2}\right) \\
& \quad+2 \mathbb{N}_{x}\left(\left(1_{\left\{L^{x_{1}}=0\right\}} \exp \left(-\lambda_{2} \frac{X_{G_{\varepsilon}} x_{2}^{x_{2}}(1)}{\varepsilon_{2}^{2}}\right) 1\left(X_{G_{\varepsilon_{2} / 2}^{x_{2}}}=0\right)-1_{\left\{L^{x_{1}}=0\right\}} 1_{\left\{L^{x_{2}}=0\right\}}\right)^{2}\right),
\end{aligned}
$$

where the last inequality is by $(a+b)^{2} \leq 2 a^{2}+2 b^{2}, \forall a, b \in \mathbb{R}$. Then we have

$$
\begin{aligned}
I^{2} & \leq 2 \mathbb{N}_{x}\left(\left(\exp \left(-\lambda_{1} \frac{X_{G_{\varepsilon_{1}}^{x_{1}}}(1)}{\varepsilon_{1}^{2}}\right) 1\left(X_{G_{\varepsilon_{1} / 2}^{x_{1}}}=0\right)-1_{\left\{L^{x_{1}}=0\right\}}\right)^{2}\right) \\
& +2 \mathbb{N}_{x}\left(\left(\exp \left(-\lambda_{2} \frac{X_{G_{\varepsilon_{2}}^{x_{2}}(1)}}{\varepsilon_{2}^{2}}\right) 1\left(X_{G_{\varepsilon_{2} / 2}^{x_{2}}}=0\right)-1_{\left\{L^{x_{2}}=0\right\}}\right)^{2}\right)=2 K_{1}+2 K_{2} \rightarrow 0
\end{aligned}
$$

as $\varepsilon_{1}, \varepsilon_{2} \downarrow 0$ where we have used (B.1) and (B.3) in the last line.

Turning to $W^{\vec{\lambda}, \vec{x}, \varepsilon}(x)$, for $\varepsilon>0$ small enough we have

$$
\begin{aligned}
& J=W^{\vec{\lambda}, \vec{x}, \varepsilon}(x)-V^{\infty, \vec{x}}(x) \\
& =\mathbb{N}_{x}\left(1-e^{-\lambda_{1} L^{x_{1}}} \exp \left(-\lambda_{2} \frac{X_{G_{\varepsilon}^{x_{2}}(1)}}{\varepsilon^{2}}\right) 1_{\left(X_{G_{\varepsilon / 2}^{x_{2}}}=0\right)}\right)-\mathbb{N}_{x}\left(1-1_{\left\{L^{x_{1}}=0\right\}} 1_{\left\{L^{x_{2}}=0\right\}}\right) \\
& =\mathbb{N}_{x}\left(1_{\left\{L^{x_{1}}=0\right\}} 1_{\left\{L^{x_{2}}=0\right\}}-e^{-\lambda_{1} L^{x_{1}}} \exp \left(-\lambda_{2} \frac{X_{G_{\varepsilon}^{x_{2}}}(1)}{\varepsilon^{2}}\right) 1\left(X_{G_{\varepsilon / 2}^{x_{2}}}=0\right)\right) .
\end{aligned}
$$


By Jensen's inequality we have

$$
\begin{aligned}
J^{2} \leq & \mathbb{N}_{x}\left(\left(e^{-\lambda_{1} L^{x_{1}}} \exp \left(-\lambda_{2} \frac{X_{G_{\varepsilon}^{x_{2}}}(1)}{\varepsilon^{2}}\right) 1\left(X_{G_{\varepsilon / 2}^{x_{2}}}=0\right)-1_{\left\{L^{x_{1}}=0\right\}} 1_{\left\{L^{\left.x_{2}=0\right\}}\right.}\right)^{2}\right) \\
\leq & 2 \mathbb{N}_{x}\left(\left(e^{-\lambda_{1} L^{x_{1}}} \exp \left(-\lambda_{2} \frac{X_{G_{\varepsilon}^{x_{2}}(1)}}{\varepsilon^{2}}\right) 1\left(X_{G_{\varepsilon / 2}^{x_{2}}}=0\right)\right.\right. \\
& \left.\left.\quad-1_{\left\{L^{x_{1}}=0\right\}} \exp \left(-\lambda_{2} \frac{X_{G_{\varepsilon}^{x_{2}}}(1)}{\varepsilon^{2}}\right) 1\left(X_{G_{\varepsilon / 2}^{x_{2}}}=0\right)\right)^{2}\right) \\
& \quad+2 \mathbb{N}_{x}\left(\left(1_{\left\{L^{x_{1}}=0\right\}} \exp \left(-\lambda_{2} \frac{X_{G_{\varepsilon}^{x_{2}}}(1)}{\varepsilon^{2}}\right) 1\left(X_{G_{\varepsilon / 2}^{x_{2}}}=0\right)-1_{\left\{L^{x_{1}}=0\right\}} 1_{\left\{L^{x_{2}}=0\right\}}\right)^{2}\right) \\
\leq & 2 \mathbb{N}_{x}\left(\left(e^{-\lambda_{1} L^{x_{1}}}-1_{\left\{L^{x_{1}}=0\right\}}\right)^{2}\right)+2 \mathbb{N}_{x}\left(\left(\exp \left(-\lambda_{2} \frac{X_{G_{\varepsilon}^{x_{2}}(1)}}{\varepsilon^{2}}\right) 1_{\left.\left(X_{\left.G_{\varepsilon / 2}^{x_{2}}=0\right)}-1_{\left\{L^{x_{2}}=0\right\}}\right)^{2}\right)}\right.\right. \\
\leq & 2 \mathbb{N}_{x}\left(e^{-2 \lambda_{1} L^{x_{1}}} 1_{\left\{L^{x_{1}}>0\right\}}\right)+2 K_{2} \rightarrow 0,
\end{aligned}
$$

as $\lambda_{1} \rightarrow \infty$ and $\varepsilon \downarrow 0$ where we have used the monotone convergence theorem and (B.3) in the last line.

\section{B.2 Proof of Lemma 8.3}

Recall $G=G_{\varepsilon_{1}}^{x_{1}} \cap G_{\varepsilon_{2}}^{x_{2}}$. For all $x \in G$ we let

$$
u(x) \equiv U^{\vec{\lambda}, \vec{x}, \vec{\varepsilon}}=\mathbb{N}_{x}\left(1-\prod_{i=1}^{2} \exp \left(-\lambda_{i} \frac{X_{G_{\varepsilon_{i}}^{x_{i}}(1)}}{\varepsilon_{i}^{2}}\right) 1\left(X_{G_{\varepsilon_{i} / 2}^{x_{i}}}=0\right)\right) .
$$

Define

$$
\widetilde{\lambda}_{i}=\lambda_{i}+4 U^{\infty, 1}(2), i=1,2
$$

and recall (8.1) to get for all $x \in G$,

$$
u(x) \leq U^{\widetilde{\lambda}_{1} \varepsilon_{1}^{-2}, \varepsilon_{1}}\left(x-x_{1}\right)+U^{\widetilde{\lambda}_{2} \varepsilon_{2}^{-2}, \varepsilon_{2}}\left(x-x_{2}\right) \leq \widetilde{\lambda}_{1} \varepsilon_{1}^{-2}+\widetilde{\lambda}_{2} \varepsilon_{2}^{-2},
$$

where the last inequality follows from that $r \mapsto U^{\lambda, \varepsilon}(r)$ is decreasing by Lemma 3.2(b) of [19] and that $U^{\lambda, \varepsilon}(\varepsilon)=\lambda$. Next, for any $x^{\prime} \in G$, let $D$ be an open ball that contains $x^{\prime}$, whose closure is in $G$. Use Proposition 2.1(i) to see that for $x \in D$,

$$
\begin{aligned}
& u(x)=\mathbb{N}_{x}\left(1-\prod_{i=1}^{2} \exp \left(-\lambda_{i} \frac{X_{G_{\varepsilon_{i}}^{x_{i}}}(1)}{\varepsilon_{i}^{2}}\right) 1\left(X_{G_{\varepsilon_{i} / 2}^{x_{i}}}=0\right)\right) \\
= & \mathbb{N}_{x}\left(1-\mathbb{E}_{X_{D}}\left(\prod_{i=1}^{2} \exp \left(-\lambda_{i} \frac{X_{G_{\varepsilon_{i}}^{x_{i}}}(1)}{\varepsilon_{i}^{2}}\right) 1\left(X_{G_{\varepsilon_{i} / 2}^{x_{i}}}=0\right)\right)\right) \\
= & \mathbb{N}_{x}\left(1-\exp \left(-\int u(y) X_{D}(d y)\right)\right),
\end{aligned}
$$

the last equality by (4.6) with $X_{0}=X_{D}$. Therefore

$$
u(x)=\mathbb{N}_{x}\left(1-\exp \left(-\int u(y) X_{D}(d y)\right)\right), \quad \forall x \in D .
$$

Note $u$ is bounded in $G$ by (B.5), and hence on $\partial D$. Use Theorem V.6 of [16] to conclude

$$
\Delta u(x)=(u(x))^{2}, \forall x \in D, \text { and, in particular, for } x=x^{\prime} .
$$

Since $x^{\prime}$ is arbitrary, it holds for all $x \in G$. 


\section{Proofs of Propositions 4.1(i) and 4.3(i)}

Proof of Proposition 4.1(i). By symmetry it suffices to consider the case $i=1$. Recall Lemma 8.2 to get

$$
\lambda_{1}^{1+\alpha} V_{1}^{\vec{\lambda}, \vec{x}}(x)=\lambda_{1}^{1+\alpha} \lim _{t \rightarrow \infty} E_{x}\left(V_{1}^{\vec{\lambda}, \vec{x}}\left(B\left(t \wedge T_{r_{\lambda}}\right)\right) \exp \left(-\int_{0}^{t \wedge T_{r_{\lambda}}} V^{\vec{\lambda}, \vec{x}}\left(B_{s}\right) d s\right)\right),
$$

where $T_{r_{\lambda}}=T_{r_{\lambda_{1}}}^{1} \wedge T_{r_{\lambda_{2}}}^{2}$ and $T_{r_{\lambda_{i}}}^{i}=\inf \left\{t \geq 0:\left|B_{t}-x_{i}\right| \leq r_{\lambda_{i}}\right\}$. Here $r_{\lambda_{i}}=\lambda_{0} \lambda_{i}^{-\frac{1}{4-d}}$ and we will choose $\lambda_{0}$ to be some fixed large constant below. By (5.2), we have $V_{1}^{\vec{\lambda}, \vec{x}}(x) \rightarrow 0$ as $|x| \rightarrow \infty$ and $V_{1}^{\vec{\lambda}, \vec{x}}\left(B\left(t \wedge T_{r_{\lambda}}\right)\right)$ is uniformly bounded for all $t \geq 0$. Apply the dominated convergence theorem to see that

$$
\begin{gathered}
\lambda_{1}^{1+\alpha} V_{1}^{\vec{\lambda}, \vec{x}}(x)=\lambda_{1}^{1+\alpha} E_{x}\left(1_{\left\{T_{r_{\lambda}}<\infty\right\}} V_{1}^{\vec{\lambda}, \vec{x}}\left(B\left(T_{r_{\lambda}}\right)\right) \exp \left(-\int_{0}^{T_{r_{\lambda}}} V^{\vec{\lambda}, \vec{x}}\left(B_{s}\right) d s\right)\right) \\
=\sum_{i=1}^{2} E_{x}\left(1_{\left\{T_{r_{\lambda_{i}}}^{i}<\infty\right\}} 1_{\left\{T_{r_{\lambda_{i}}}^{i}<T_{r_{\lambda_{3} i}}^{3-i}\right\}} \lambda_{1}^{1+\alpha} V_{1}^{\vec{\lambda}, \vec{x}}\left(B\left(T_{r_{\lambda_{i}}}^{i}\right)\right)\right. \\
\left.\exp \left(-\int_{0}^{T_{r_{\lambda_{i}}}^{i}} V^{\vec{\lambda}, \vec{x}}\left(B_{s}\right) d s\right)\right):=I_{1}+I_{2} .
\end{gathered}
$$

We first deal with $I_{2}$. Note in the integrand of $I_{2}$ we may assume that $\left|B\left(T_{r_{\lambda_{2}}}^{2}\right)-x_{2}\right|=r_{\lambda_{2}}$ and so by (8.9) we have $\left|x_{1}-B\left(T_{r_{\lambda_{2}}}^{2}\right)\right|>\Delta / 2$ where $\Delta=\left|x_{1}-x_{2}\right|$. Apply (5.2) with $x=B\left(T_{r_{\lambda_{2}}}^{2}\right)$ to get

$$
\lambda_{1}^{1+\alpha} V_{1}^{\vec{\lambda}, \vec{x}}\left(B\left(T_{r_{\lambda_{2}}}^{2}\right)\right) \leq c_{3.2}\left|B\left(T_{r_{\lambda_{2}}}^{2}\right)-x_{1}\right|^{-p} \leq c_{3.2} \Delta^{-p} 2^{p} .
$$

Let $\tau_{r}=\inf \left\{t \geq 0:\left|B_{t}\right| \leq r\right\}$ and use (C.2) and (8.4) to see that $I_{2}$ becomes

$$
\begin{aligned}
I_{2} & \leq c_{3.2} 2^{p} \Delta^{-p} E_{x}\left(1_{\left\{T_{r_{\lambda_{2}}}^{2}<\infty\right\}} 1_{\left\{T_{r_{\lambda_{2}}}^{2}<T_{r_{\lambda_{1}}}^{1}\right\}} \exp \left(-\int_{0}^{T_{r_{\lambda_{2}}}^{2}} V^{\vec{\lambda}, \vec{x}}\left(B_{s}\right) d s\right)\right) \\
& \leq c_{3.2} 2^{p} \Delta^{-p} E_{x-x_{2}}\left(1_{\left\{\tau_{r_{2}}<\infty\right\}} \exp \left(-\int_{0}^{\tau_{r_{\lambda_{2}}}} V^{\lambda_{2}}\left(B_{s}\right) d s\right)\right) \\
& =c_{3.2} 2^{p} \Delta^{-p} r_{\lambda_{2}}^{p}\left|x-x_{2}\right|^{-p} E_{\left|x-x_{2}\right|}^{(2+2 \nu)}\left(\exp \left(\int_{0}^{\tau_{r_{\lambda_{2}}}}\left(V^{\infty}-V^{\lambda_{2}}\right)\left(\rho_{s}\right) d s\right) \mid \tau_{r_{\lambda_{2}}}<\infty\right) \\
& \leq c_{3.2} 2^{p} \Delta^{-p} r_{\lambda_{2}}^{p}\left|x-x_{2}\right|^{-p} C_{3.5}\left(\lambda_{0}, \nu, 1\right) \rightarrow 0 \text { as } \lambda_{2} \rightarrow \infty,
\end{aligned}
$$

where we have used Proposition 3.7 in the equality and we choose $\lambda_{0}>c_{3.5}$ to apply Lemma 3.5 in the last inequality.

Now we will turn to $I_{1}$. Let $\left(Y_{t}, t \geq 0\right)$ be the $d$-dimensional coordinate process under Wiener measure, $P_{x}$. By slightly abusing the notation, we set $\tau_{r}=\tau_{r}^{Y}=\inf \left\{t \geq 0:\left|Y_{t}\right| \leq\right.$ $r$ for any $r>0$, and set

$$
T_{r_{\lambda_{2}}}^{\prime}=T_{r_{\lambda_{2}}}^{\prime, Y}=\inf \left\{t \geq 0:\left|Y_{t}-\left(x_{2}-x_{1}\right)\right| \leq r_{\lambda_{2}}\right\}
$$

Then use translation invariance of $Y$ to get

$$
I_{1}=E_{x-x_{1}}\left(1_{\left\{\tau_{r_{\lambda_{1}}}<\infty\right\}} 1_{\left\{\tau_{r_{\lambda_{1}}}<T_{r_{\lambda_{2}}}^{\prime}\right.} \lambda_{1}^{1+\alpha} V_{1}^{\vec{\lambda}, \vec{x}}\left(Y\left(\tau_{r_{\lambda_{1}}}\right)+x_{1}\right) \exp \left(-\int_{0}^{\tau_{r_{\lambda_{1}}}} V^{\vec{\lambda}, \vec{x}}\left(Y_{s}+x_{1}\right) d s\right)\right) .
$$

Recall that $\hat{P}_{x}^{(2-2 \nu)}$ is the law of $Y$ starting from $x$ such that $Y$ is the unique solution of

$$
\left\{\begin{array}{l}
Y_{t}=x+\hat{B}_{t}+\int_{0}^{t}(-\nu-\mu) \frac{Y_{s}}{\left|Y_{s}\right|^{2}} d s, \quad t<\tau_{0}, \\
Y_{t}=0, t \geq \tau_{0},
\end{array}\right.
$$


where $\hat{B}$ is a standard $d$-dimensional Brownian motion under $\hat{P}_{x}^{(2-2 \nu)}$. Apply Proposition 7.1 with $g(\cdot)=V^{\vec{\lambda}, \vec{x}}\left(\cdot+x_{1}\right)$ in the above to get

$$
\begin{aligned}
I_{1}=\frac{r_{\lambda_{1}}^{p}}{\left|x-x_{1}\right|^{p}} \hat{E}_{x-x_{1}}^{(2-2 \nu)}\left(1_{\left\{\tau_{r_{\lambda_{1}}}<T_{r_{\lambda_{2}}}^{\prime}\right\}} \lambda_{1}^{1+\alpha} V_{1}^{\vec{\lambda}, \vec{x}}\left(Y\left(\tau_{r_{\lambda_{1}}}\right)+x_{1}\right)\right. \\
\left.\quad \times \exp \left(-\int_{0}^{\tau_{r_{\lambda_{1}}}}\left(V^{\vec{\lambda}, \vec{x}}\left(Y_{s}+x_{1}\right)-V^{\infty}\left(Y_{s}\right)\right) d s\right)\right) \\
=\frac{1}{\left|x-x_{1}\right|^{p}} \hat{E}_{x-x_{1}}^{(2-2 \nu)}\left(\left[1_{\left.\left\{\tau_{r_{\lambda_{1}}}<T_{r_{\lambda_{2}}}^{\prime}\right\}\right]\left[r_{\lambda_{1}}^{p} \lambda_{1}^{1+\alpha} V_{1}^{\vec{\lambda}, \vec{x}}\left(Y\left(\tau_{r_{\lambda_{1}}}\right)+x_{1}\right)\right]}\right.\right. \\
\quad \times\left[\exp \left(-\int_{0}^{\tau_{r_{\lambda_{1}}}}\left(V^{\vec{\lambda}, \vec{x}}\left(Y_{s}+x_{1}\right)-V^{\lambda_{1}}\left(Y_{s}\right)\right) d s\right)\right] \\
\left.\quad \times\left[\exp \left(\int_{0}^{\tau_{r_{\lambda_{1}}}}\left(V^{\infty}\left(Y_{s}\right)-V^{\lambda_{1}}\left(Y_{s}\right)\right) d s\right)\right]\right) \\
:=\frac{1}{\left|x-x_{1}\right|^{p}} \hat{E}_{x-x_{1}}^{(2-2 \nu)}\left(\left[\widetilde{\left.J_{1}\right]}\left[\widetilde{J_{2}}\right]\left[\widetilde{J_{3}}\right]\left[\widetilde{J_{4}}\right]\right),\right.
\end{aligned}
$$

where we have ordered the fours terms in square brackets as $\widetilde{J_{1}}, \ldots, \widetilde{J_{4}}$.

We first consider $\widetilde{J_{2}}$. Recall (4.9) and use translation invariance to get

$$
\begin{aligned}
\widetilde{J_{2}} & =r_{\lambda_{1}}^{p} \lambda_{1}^{1+\alpha} \mathbb{N}_{Y\left(\tau_{r_{\lambda_{1}}}\right)+x_{1}}\left(L^{x_{1}} \exp \left(-\lambda_{1} L^{x_{1}}-\lambda_{2} L^{x_{2}}\right)\right) \\
& =r_{\lambda_{1}}^{p} \lambda_{1}^{1+\alpha} \mathbb{N}_{Y\left(\tau_{r_{\lambda_{1}}}\right)}\left(L^{0} \exp \left(-\lambda_{1} L^{0}-\lambda_{2} L^{x_{2}-x_{1}}\right)\right) .
\end{aligned}
$$

By the scaling of Brownian snake and its local time $\left(L^{x_{0}}\right)$ under the excursion measure $\mathbb{N}_{x}$ (see, e.g., Proof of Proposition V.9 in [16]), we have

$$
\begin{aligned}
\widetilde{J_{2}} & =r_{\lambda_{1}}^{p} \lambda_{1}^{1+\alpha} r_{\lambda_{1}}^{-2} \mathbb{N}_{Y\left(\tau_{\lambda_{1}}\right) / r_{\lambda_{1}}}\left(r_{\lambda_{1}}^{4-d} L^{0} e^{-\lambda_{1} r_{\lambda_{1}}^{4-d} L^{0}} e^{-\lambda_{2} r_{\lambda_{1}}^{4-d} L^{\left(x_{2}-x_{1}\right) / r_{\lambda_{1}}}}\right) \\
& =\lambda_{0}^{p+2-d} \mathbb{N}_{Y\left(\tau_{r_{1}}\right) / r_{\lambda_{1}}}\left(L^{0} e^{-\lambda_{0}^{4-d} L^{0}} e^{-\lambda_{2} r_{\lambda_{1}}^{4-d} L^{\left(x_{2}-x_{1}\right) / r_{\lambda_{1}}}}\right) \\
& \stackrel{\text { law }}{=} \lambda_{0}^{p+2-d} \mathbb{N}_{Y\left(\tau_{1}\right)}\left(L^{0} e^{-\lambda_{0}^{4-d} L^{0}} e^{-\lambda_{2} r_{\lambda_{1}}^{4-d} L^{\left(x_{2}-x_{1}\right) / r_{\lambda_{1}}}}\right),
\end{aligned}
$$

where in the next to last equality we have used the definitions of $r_{\lambda_{1}}$ and $\alpha$ and the last equality follows from the scaling of $Y$. Note for any $K>0$, we have

$$
\left|\frac{x_{2}-x_{1}}{r_{\lambda_{1}}}\right|>K \text { for } \lambda_{1} \text { large enough, }
$$

and so by the compactness of the support of SBM (see (2.3)), we conclude $\mathbb{N}_{Y\left(\tau_{1}\right)}$-a.e.,

$$
L^{\left(x_{2}-x_{1}\right) / r_{\lambda_{1}}}=0 \text { for } \lambda_{1} \text { large enough. }
$$

Therefore an application of the dominated convergence theorem Theorem will give us

$$
\begin{aligned}
& \lim _{\lambda_{1}, \lambda_{2} \rightarrow \infty} \mathbb{N}_{Y\left(\tau_{1}\right)}\left(L^{0} \exp \left(-\lambda_{0}^{4-d} L^{0}-\lambda_{2} r_{\lambda_{1}}^{4-d} L^{\left(x_{2}-x_{1}\right) / r_{\lambda_{1}}}\right)\right), \\
= & \mathbb{N}_{Y\left(\tau_{1}\right)}\left(L^{0} \exp \left(-\lambda_{0}^{4-d} L^{0}\right)\right)=\mathbb{N}_{e_{1}}\left(L^{0} \exp \left(-\lambda_{0}^{4-d} L^{0}\right)\right)=V_{1}^{\lambda_{0}^{4-d}}(1),
\end{aligned}
$$

where in the next to last equality we have used spherical symmetry and $e_{1}$ is the first unit basis vector. The last equality follows by (3.2). In view of (C.7), we have proved

$$
\widetilde{J_{2}}=r_{\lambda_{1}}^{p} \lambda_{1}^{1+\alpha} V_{1}^{\vec{\lambda}, \vec{x}}\left(Y\left(\tau_{r_{\lambda_{1}}}\right)+x_{1}\right) \stackrel{d}{\rightarrow} \lambda_{0}^{p+2-d} V_{1}^{\lambda_{0}^{4-d}}(1) \text { in distribution }
$$


as $\lambda_{1}, \lambda_{2} \rightarrow \infty$, and furthermore, under $\hat{P}_{x-x_{1}}^{(2-2 \nu)}$ we have

$$
\widetilde{J_{2}}=r_{\lambda_{1}}^{p} \lambda_{1}^{1+\alpha} V_{1}^{\vec{\lambda}, \vec{x}}\left(Y\left(\tau_{r_{\lambda_{1}}}\right)+x_{1}\right) \rightarrow \lambda_{0}^{p+2-d} V_{1}^{\lambda_{0}^{4-d}}(1) \text { in probability }
$$

as $\lambda_{1}, \lambda_{2} \rightarrow \infty$ since $\lambda_{0}^{p+2-d} V_{1}^{\lambda_{0}^{4-d}}(1)$ is a constant.

By (8.20), with $\hat{P}_{x-x_{1}}^{(2-2 \nu)}$-probability one we have

$$
\widetilde{J_{1}}=1_{\left\{\tau_{r_{\lambda_{1}}}<T_{r_{\lambda_{2}}}^{\prime}\right\}} \rightarrow 1 \text { as } \lambda_{1}, \lambda_{2} \rightarrow \infty
$$

As for (8.21), we use Lemma 8.1 to see that with $\hat{P}_{x-x_{1}}^{(2-2 \nu)}$-probability one,

$$
\begin{aligned}
\widetilde{J_{3}}=\exp (- & \left.\int_{0}^{\tau_{\lambda_{\lambda_{1}}}}\left(V^{\vec{\lambda}, \vec{x}}\left(Y_{s}+x_{1}\right)-V^{\lambda_{1}}\left(Y_{s}\right)\right) d s\right) \\
& \rightarrow \exp \left(-\int_{0}^{\tau_{0}}\left(V^{\vec{\infty}, \vec{x}}\left(Y_{s}+x_{1}\right)-V^{\infty}\left(Y_{s}\right)\right) d s\right) \text { as } \lambda_{1}, \lambda_{2} \rightarrow \infty .
\end{aligned}
$$

Here one can see from (8.4) that

$$
0 \leq V^{\vec{\lambda}, \vec{x}}\left(Y_{s}+x_{1}\right)-V^{\lambda_{1}}\left(Y_{s}\right) \leq V^{\lambda_{2}}\left(Y_{s}-\left(x_{2}-x_{1}\right)\right) \leq V^{\infty}\left(Y_{s}-\left(x_{2}-x_{1}\right)\right),
$$

and so apply the dominated convergence theorem as before.

Combining (C.10), (C.11) and (C.12), we conclude that under $\hat{P}_{x-x_{1}}^{(2-2 \nu)}$

$$
\begin{gathered}
\widetilde{J_{1}} \widetilde{J_{2}} \widetilde{J_{3}} \rightarrow \lambda_{0}^{p+2-d} V_{1}^{\lambda_{0}^{4-d}}(1) \exp \left(-\int_{0}^{\tau_{0}}\left(V^{\vec{\infty}, \vec{x}}\left(Y_{s}+x_{1}\right)-V^{\infty}\left(Y_{s}\right)\right) d s\right) \\
\text { in probability as } \lambda_{1}, \lambda_{2} \rightarrow \infty .
\end{gathered}
$$

Recall (5.2) to see that

$$
\widetilde{J_{2}}=r_{\lambda_{1}}^{p} \lambda_{1}^{1+\alpha} V_{1}^{\vec{\lambda}, \vec{x}}\left(Y\left(\tau_{r_{\lambda_{1}}}\right)+x_{1}\right) \leq r_{\lambda_{1}}^{p} c_{3.2}\left|Y\left(\tau_{r_{\lambda_{1}}}\right)\right|^{-p}=c_{3.2} .
$$

Use (8.4) to see that $\widetilde{J_{3}} \leq 1$ and so conclude

$$
\widetilde{J_{1}} \widetilde{J_{2}} \widetilde{J_{3}} \leq c_{3.2}, \quad \hat{P}_{x-x_{1}}^{(2-2 \nu)} \text {-a.s. }
$$

Recall (8.32) and use (C.13), (C.14) and the bounded convergence theorem to get

$$
\begin{aligned}
\lim _{\lambda_{1}, \lambda_{2} \rightarrow \infty} \hat{E}_{x-x_{1}}^{(2-2 \nu)}( & \left(\lambda_{0}^{p+2-d} V_{1}^{\lambda_{0}^{4-d}}(1) \exp \left(-\int_{0}^{\tau_{0}}\left(V^{\vec{\infty}, \vec{x}}\left(Y_{s}+x_{1}\right)-V^{\infty}\left(Y_{s}\right)\right) d s\right)\right. \\
& \left.\left.-\widetilde{J_{1}} \widetilde{J_{2}} \widetilde{J_{3}}\right)^{2}\right)=0 .
\end{aligned}
$$

Recalling $\widetilde{J_{4}}$ as in (C.6), we use the fact that under $\hat{P}_{x-x_{1}}^{(2-2 \nu)}$, the process $\left\{\left|Y_{s \wedge \tau_{r_{\lambda_{1}}}}\right|, s \geq 0\right\}$ is a stopped $(2-2 \nu)$-dimensional Bessel process and then use Corollary 7.3 to get for all $\lambda_{1}>0$,

$$
\begin{aligned}
& \hat{E}_{x-x_{1}}^{(2-2 \nu)}\left({\widetilde{J_{4}}}^{2}\right)=\hat{E}_{x-x_{1}}^{(2-2 \nu)}\left(\exp \left(2 \int_{0}^{\tau_{r_{\lambda_{1}}}}\left(V^{\infty}\left(Y_{s}\right)-V^{\lambda_{1}}\left(Y_{s}\right)\right) d s\right)\right) \\
= & E_{\left|x-x_{1}\right|}^{(2-2 \nu)}\left(\exp \left(2 \int_{0}^{\tau_{r_{\lambda_{1}}}}\left(V^{\infty}\left(\rho_{s}\right)-V^{\lambda}\left(\rho_{s}\right)\right) d s\right)\right) \\
= & E_{\left|x-x_{1}\right|}^{(2+2 \nu)}\left(\exp \left(2 \int_{0}^{\tau_{r_{\lambda_{1}}}}\left(V^{\infty}\left(\rho_{s}\right)-V^{\lambda}\left(\rho_{s}\right)\right) d s\right) \mid \tau_{r_{\lambda_{1}}}<\infty\right) \\
\leq & C_{3.5}\left(\lambda_{0}, \nu, 2\right)<\infty
\end{aligned}
$$


where we have chosen $\lambda_{0}>c_{3.5}$ so that we can apply Lemma 3.5 in the last inequality. Now we can conclude that

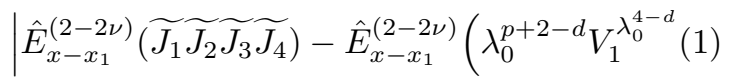

$$
\begin{aligned}
& \left.\times \exp \left(-\int_{0}^{\tau_{0}}\left(V^{\vec{\infty}, \vec{x}}\left(Y_{s}+x_{1}\right)-V^{\infty}\left(Y_{s}\right)\right) d s\right) \times \widetilde{J_{4}}\right) \mid \\
& \leq \hat{E}_{\left|x-x_{1}\right|}^{(2-2 \nu)}\left(\widetilde{J_{4}} \times \mid \widetilde{J_{1}} \widetilde{J_{2}} \widetilde{J_{3}}-\lambda_{0}^{p+2-d} V_{1}^{\lambda_{0}^{4-d}}(1)\right. \\
& \left.\times \exp \left(-\int_{0}^{\tau_{0}}\left(V^{\vec{\infty}, \vec{x}}\left(Y_{s}+x_{1}\right)-V^{\infty}\left(Y_{s}\right)\right) d s\right) \mid\right) \\
& \leq\left(\hat{E}_{x-x_{1}}^{(2-2 \nu)}\left(\widetilde{J}_{4}^{2}\right)\right)^{1 / 2}\left(\hat { E } _ { x - x _ { 1 } } ^ { ( 2 - 2 \nu ) } \left(\widetilde{J_{1}} \widetilde{J_{2}} \widetilde{J_{3}}-\lambda_{0}^{p+2-d} V_{1}^{\lambda_{0}^{4-d}}(1)\right.\right. \\
& \left.\left.\times \exp \left(-\int_{0}^{\tau_{0}}\left(V^{\vec{\infty}, \vec{x}}\left(Y_{s}+x_{1}\right)-V^{\infty}\left(Y_{s}\right)\right) d s\right)\right)^{2}\right)^{1 / 2} \rightarrow 0
\end{aligned}
$$

as $\lambda_{1}, \lambda_{2} \rightarrow \infty$, where the second inequality is by the Cauchy-Schwartz inequality and the convergence to 0 follows from (C.15) and (C.16). In view of (C.6), we conclude

$$
\begin{aligned}
& \lim _{\lambda_{1}, \lambda_{2} \rightarrow \infty} I_{1}=\frac{\lambda_{0}^{p+2-d} V_{1}^{\lambda_{0}^{4-d}}(1)}{\left|x-x_{1}\right|^{p}} \\
& \lim _{\lambda_{1}, \lambda_{2} \rightarrow \infty} \hat{E}_{x-x_{1}}^{(2-2 \nu)}\left(\exp \left(-\int_{0}^{\tau_{0}}\left(V^{\vec{\infty}, \vec{x}}\left(Y_{s}+x_{1}\right)-V^{\infty}\left(Y_{s}\right)\right) d s\right) \cdot \widetilde{J_{4}}\right)
\end{aligned}
$$

provided we can show the limit on the right-hand side exists.

Recall $C_{3.5}\left(\lambda_{0}, \nu, 1\right)$ as in Lemma 3.5. We claim that

$$
\begin{aligned}
& \lim _{\lambda_{1} \rightarrow \infty} \hat{E}_{x-x_{1}}^{(2-2 \nu)}\left(\exp \left(-\int_{0}^{\tau_{0}}\left(V^{\vec{\infty}, \vec{x}}\left(Y_{s}+x_{1}\right)-V^{\infty}\left(Y_{s}\right)\right) d s\right) \cdot \widetilde{J_{4}}\right) \\
= & C_{3.5}\left(\lambda_{0}, \nu, 1\right) \hat{E}_{x-x_{1}}^{(2-2 \nu)}\left(\exp \left(-\int_{0}^{\tau_{0}}\left(V^{\vec{\infty}, \vec{x}}\left(Y_{s}+x_{1}\right)-V^{\infty}\left(Y_{s}\right)\right) d s\right)\right) .
\end{aligned}
$$

It will then follow from (C.1), (C.3), (C.17) and (C.18) that

$$
\begin{aligned}
& \lim _{\lambda_{1}, \lambda_{2} \rightarrow \infty} \lambda_{1}^{1+\alpha} V_{1}^{\vec{\lambda}, \vec{x}}(x)=\lambda_{0}^{p+2-d} V_{1}^{\lambda_{0}^{4-d}}(1) C_{3.5}\left(\lambda_{0}, \nu, 1\right) \\
& \quad\left|x-x_{1}\right|^{-p} \hat{E}_{x-x_{1}}^{(2-2 \nu)}\left(\exp \left(-\int_{0}^{\tau_{0}}\left(V^{\vec{\infty}, \vec{x}}\left(Y_{s}+x_{1}\right)-V^{\infty}\left(Y_{s}\right)\right) d s\right)\right),
\end{aligned}
$$

and the proof will be complete by letting $K_{4.1}=\lambda_{0}^{p+2-d} V_{1}^{\lambda_{0}^{4-d}}(1) C_{3.5}\left(\lambda_{0}, \nu, 1\right)$. Recall $c_{3.8}$ as in Lemma 3.8 (see (3.11)) to conclude $K_{4.1}=c_{3.8}$.

It remains to prove (C.18). First by (8.32) and the monotone convergence theorem, we have

$$
\begin{aligned}
\lim _{\delta \rightarrow 0} \mid & \hat{E}_{x-x_{1}}^{(2-2 \nu)}\left(\exp \left(-\int_{0}^{\tau_{\delta}}\left(V^{\vec{\infty}, \vec{x}}\left(Y_{s}+x_{1}\right)-V^{\infty}\left(Y_{s}\right)\right) d s\right)\right) \\
& -\hat{E}_{x-x_{1}}^{(2-2 \nu)}\left(\exp \left(-\int_{0}^{\tau_{0}}\left(V^{\vec{\infty}, \vec{x}}\left(Y_{s}+x_{1}\right)-V^{\infty}\left(Y_{s}\right)\right) d s\right)\right) \mid=0 .
\end{aligned}
$$


Since $\widetilde{J_{4}}$ has an uniform $L^{2}$ bound for all $\lambda_{1}>0$ by (C.16), by Cauchy-Schwartz we have

$$
\begin{aligned}
& \mid \hat{E}_{x-x_{1}}^{(2-2 \nu)}\left(\exp \left(-\int_{0}^{\tau_{\delta}}\left(V^{\vec{\infty}, \vec{x}}\left(Y_{s}+x_{1}\right)-V^{\infty}\left(Y_{s}\right)\right) d s\right) \times \widetilde{J_{4}}\right) \\
& -\hat{E}_{x-x_{1}}^{(2-2 \nu)}\left(\exp \left(-\int_{0}^{\tau_{0}}\left(V^{\vec{\infty}, \vec{x}}\left(Y_{s}+x_{1}\right)-V^{\infty}\left(Y_{s}\right)\right) d s\right) \times \widetilde{J_{4}}\right) \mid \\
\leq & \left(\hat{E}_{x-x_{1}}^{(2-2 \nu)}\left({\widetilde{J_{4}}}_{4}^{2}\right)\right)^{1 / 2}\left(\hat { E } _ { x - x _ { 1 } } ^ { ( 2 - 2 \nu ) } \left(\exp \left(-\int_{0}^{\tau_{\delta}}\left(V^{\vec{\infty}, \vec{x}}\left(Y_{s}+x_{1}\right)-V^{\infty}\left(Y_{s}\right)\right) d s\right)\right.\right. \\
& \left.\left.-\exp \left(-\int_{0}^{\tau_{0}}\left(V^{\vec{\infty}, \vec{x}}\left(Y_{s}+x_{1}\right)-V^{\infty}\left(Y_{s}\right)\right) d s\right)\right)^{2}\right)^{1 / 2} \rightarrow 0 \text { as } \delta \downarrow 0
\end{aligned}
$$

uniformly for all $\lambda_{1}>0$, where the last follows from the monotone convergence theorem and (C.16). Fixing any $\delta>0$, we will show that

$$
\begin{aligned}
& \lim _{\lambda_{1} \rightarrow \infty} \hat{E}_{x-x_{1}}^{(2-2 \nu)}\left(\exp \left(-\int_{0}^{\tau_{\delta}}\left(V^{\vec{\infty}, \vec{x}}\left(Y_{s}+x_{1}\right)-V^{\infty}\left(Y_{s}\right)\right) d s\right) \cdot \widetilde{J_{4}}\right) \\
= & C_{3.5}\left(\lambda_{0}, \nu, 1\right) \hat{E}_{x-x_{1}}^{(2-2 \nu)}\left(\exp \left(-\int_{0}^{\tau_{\delta}}\left(V^{\vec{\infty}, \vec{x}}\left(Y_{s}+x_{1}\right)-V^{\infty}\left(Y_{s}\right)\right) d s\right)\right),
\end{aligned}
$$

and one can easily conclude from (C.19), (C.20) and (C.21) that (C.18) holds.

It remains to prove (C.21). For $r_{\lambda_{1}}<\delta$ we use the strong Markov property of $\left(Y_{s}, s \geq 0\right)$ to get

$$
\begin{aligned}
\hat{E}_{x-x_{1}}^{(2-2 \nu)}\left(\exp \left(-\int_{0}^{\tau_{\delta}}\left(V^{\vec{\infty}, \vec{x}}\left(Y_{s}+x_{1}\right)-V^{\infty}\left(Y_{s}\right)\right) d s\right)\right. \\
\left.\times \exp \left(\int_{0}^{\tau_{r_{1}}}\left(V^{\infty}-V^{\lambda_{1}}\right)\left(Y_{s}\right) d s\right)\right) \\
=\hat{E}_{x-x_{1}}^{(2-2 \nu)}\left(\exp \left(-\int_{0}^{\tau_{\delta}}\left(V^{\vec{\infty}, \vec{x}}\left(Y_{s}+x_{1}\right)-V^{\infty}\left(Y_{s}\right)\right) d s\right)\right. \\
\times \exp \left(\int_{0}^{\tau_{\delta}}\left(V^{\infty}\left(Y_{s}\right)-V^{\lambda_{1}}\left(Y_{s}\right)\right) d s\right) \\
\left.\times \hat{E}_{Y_{\tau_{\delta}}}^{(2-2 \nu)}\left(\exp \left(\int_{0}^{\tau_{\lambda_{\lambda_{1}}}}\left(V^{\infty}\left(Y_{s}\right)-V^{\lambda_{1}}\left(Y_{s}\right)\right) d s\right)\right)\right) .
\end{aligned}
$$

Using Corollary 7.3 as in (C.16), we have

$$
\begin{aligned}
& \hat{E}_{Y_{\tau_{\delta}}}^{(2-2 \nu)}\left(\exp \left(\int_{0}^{\tau_{r_{\lambda_{1}}}}\left(V^{\infty}\left(Y_{s}\right)-V^{\lambda_{1}}\left(Y_{s}\right)\right) d s\right)\right) \\
& =E_{\left|Y_{\tau_{\delta}}\right|}^{(2-2 \nu)}\left(\exp \left(\int_{0}^{\tau_{r_{\lambda_{1}}}}\left(V^{\infty}\left(\rho_{s}\right)-V^{\lambda_{1}}\left(\rho_{s}\right)\right) d s\right)\right) \\
& =E_{\delta}^{(2+2 \nu)}\left(\exp \left(\int_{0}^{\tau_{r_{\lambda_{1}}}}\left(V^{\infty}\left(\rho_{s}\right)-V^{\lambda_{1}}\left(\rho_{s}\right)\right) d s\right) \mid \tau_{r_{\lambda_{1}}}<\infty\right) \\
& \uparrow C_{3.5}\left(\lambda_{0}, \nu, 1\right) \text { as } \lambda_{1} \rightarrow \infty,
\end{aligned}
$$

where the last follows from Lemma 3.5 by choosing $\lambda_{0}>c_{3.5}$. Next since $\delta>0$ is fixed, we have

$$
\lim _{\lambda_{1} \rightarrow \infty} \exp \left(\int_{0}^{\tau_{\delta}}\left(V^{\infty}\left(Y_{s}\right)-V^{\lambda_{1}}\left(Y_{s}\right)\right) d s\right) \rightarrow 1, \hat{P}_{x-x_{1}}^{(2-2 \nu)} \text {-a.s. }
$$


In view of (C.23) and (8.32), with $\hat{P}_{x-x_{1}}^{(2-2 \nu)}$-probability one, for any $\lambda_{1}>0$ we have

$$
\begin{aligned}
& \exp \left(-\int_{0}^{\tau_{\delta}}\left(V^{\vec{\infty}, \vec{x}}\left(Y_{s}+x_{1}\right)-V^{\infty}\left(Y_{s}\right)\right) d s\right) \\
& \times \exp \left(\int_{0}^{\tau_{\delta}}\left(V^{\infty}\left(Y_{s}\right)-V^{\lambda_{1}}\left(Y_{s}\right)\right) d s\right) \\
& \times \hat{E}_{Y_{\tau_{\delta}}}^{(2-2 \nu)}\left(\exp \left(\int_{0}^{\tau_{r_{\lambda_{1}}}}\left(V^{\infty}\left(Y_{s}\right)-V^{\lambda_{1}}\left(Y_{s}\right)\right) d s\right)\right) \\
& \leq \exp \left(\int_{0}^{\tau_{\delta}} V^{\infty}\left(Y_{s}\right) d s\right) \cdot C_{3.5}\left(\lambda_{0}, \nu, 1\right) .
\end{aligned}
$$

Similar to (C.23), we apply Corollary 7.3 and Lemma 3.4(i) to get

$$
\begin{aligned}
& \hat{E}_{x-x_{1}}^{(2-2 \nu)}\left(\exp \left(\int_{0}^{\tau_{\delta}} V^{\infty}\left(Y_{s}\right) d s\right)\right)=E_{\left|x-x_{1}\right|}^{(2-2 \nu)}\left(\exp \left(\int_{0}^{\tau_{\delta}} V^{\infty}\left(\rho_{s}\right) d s\right)\right) \\
= & E_{\left|x-x_{1}\right|}^{(2+2 \nu)}\left(\exp \left(\int_{0}^{\tau_{\delta}} V^{\infty}\left(\rho_{s}\right) d s\right) \mid \tau_{\delta}<\infty\right) \\
= & E_{\left|x-x_{1}\right| / \delta}^{(2+2 \nu)}\left(\exp \left(\int_{0}^{\tau_{1}} V^{\infty}\left(\rho_{s}\right) d s\right) \mid \tau_{1}<\infty\right)=\left(\left|x-x_{1}\right| / \delta\right)^{\nu-\mu}<\infty,
\end{aligned}
$$

where the second last equality is by scaling of Bessel process. Combine (C.23)-(C.26) to see that the integrand in (C.22) converges pointwise a.s. and is bounded by (the integrable) $\exp \left(\int_{0}^{\tau_{\delta}} V^{\infty}\left(Y_{s}\right) d s\right) \cdot C_{3.5}\left(\lambda_{0}, \nu, 1\right)$. Therefore by the dominated convergence theorem we conclude that

$$
\begin{aligned}
& \lim _{\lambda_{1} \rightarrow \infty} \hat{E}_{x-x_{1}}^{(2-2 \nu)}( \exp \left(-\int_{0}^{\tau_{\delta}}\left(V^{\vec{\infty}, \vec{x}}\left(Y_{s}+x_{1}\right)-V^{\infty}\left(Y_{s}\right)\right) d s\right) \\
&\left.\times \exp \left(\int_{0}^{\tau_{r_{\lambda_{1}}}}\left(V^{\infty}-V^{\lambda_{1}}\right)\left(Y_{s}\right) d s\right)\right) \\
&=C_{3.5}\left(\lambda_{0}, \nu, 1\right) \hat{E}_{x-x_{1}}^{(2-2 \nu)}\left(\exp \left(-\int_{0}^{\tau_{\delta}}\left(V^{\vec{\infty}, \vec{x}}\left(Y_{s}+x_{1}\right)-V^{\infty}\left(Y_{s}\right)\right) d s\right)\right),
\end{aligned}
$$

and the proof of (C.21) is complete.

Proof of Proposition 4.3(i). We will only give the convergence of $\lambda_{1}^{1+\alpha} W_{1}^{\vec{\lambda}, \vec{x}, \varepsilon}(x)$ and leave the details for the convergence of $\frac{1}{\varepsilon^{p-2}} W_{2}^{\vec{\lambda}, \vec{x}, \varepsilon}(x)$ to the readers. Recall Lemma 8.6 to see that

$$
\lambda_{1}^{1+\alpha} W_{1}^{\vec{\lambda}, \vec{x}, \varepsilon}(x)=\lambda_{1}^{1+\alpha} \lim _{t \rightarrow \infty} E_{x}\left(W_{1}^{\vec{\lambda}, \vec{x}, \varepsilon}\left(B\left(t \wedge T_{\lambda_{1}, \varepsilon}\right)\right) \exp \left(-\int_{0}^{t \wedge T_{\lambda_{1}, \varepsilon}} W^{\vec{\lambda}, \vec{x}, \varepsilon}\left(B_{s}\right) d s\right)\right),
$$

where $T_{\lambda_{1}, \varepsilon}=T_{r_{\lambda_{1}}}^{1} \wedge T_{2 \varepsilon}^{2}$ and $T_{r_{\lambda_{1}}}^{1}=\inf \left\{t \geq 0:\left|B_{t}-x_{1}\right| \leq r_{\lambda_{1}}\right\}$ and $T_{2 \varepsilon}^{2}=\inf \{t \geq 0$ : $\left.\left|B_{t}-x_{2}\right| \leq 2 \varepsilon\right\}$. Here $r_{\lambda_{1}}=\lambda_{0} \lambda_{1}^{-\frac{1}{4-d}}$ and we will choose $\lambda_{0}$ to be some fixed large constant below. By (5.4), we have $W_{1}^{\vec{\lambda}, \vec{x}, \varepsilon}(x) \rightarrow 0$ as $|x| \rightarrow \infty$ and $W_{1}^{\vec{\lambda}, \vec{x}, \varepsilon}\left(B\left(t \wedge T_{r_{\lambda}, \varepsilon}\right)\right)$ is uniformly bounded for all $t \geq 0$. Apply the dominated convergence theorem to get

$$
\begin{aligned}
& \lambda_{1}^{1+\alpha} W_{1}^{\vec{\lambda}, \vec{x}, \varepsilon}(x) \\
= & \lambda_{1}^{1+\alpha} E_{x}\left(1_{\left\{T_{\lambda_{1}, \varepsilon}<\infty\right\}} W_{1}^{\vec{\lambda}, \vec{x}, \varepsilon}\left(B\left(T_{\lambda_{1}, \varepsilon}\right)\right) \exp \left(-\int_{0}^{T_{\lambda_{1}, \varepsilon}} W^{\vec{\lambda}, \vec{x}, \varepsilon}\left(B_{s}\right) d s\right)\right) \\
= & E_{x}\left(1_{\left\{T_{r_{\lambda_{1}}}^{1}<\infty\right\}} 1_{\left\{T_{r_{\lambda_{1}}}^{1}<T_{2 \varepsilon}^{2}\right\}} \lambda_{1}^{1+\alpha} W_{1}^{\vec{\lambda}, \vec{x}, \varepsilon}\left(B\left(T_{r_{\lambda_{1}}}^{1}\right)\right) \exp \left(-\int_{0}^{T_{r_{\lambda_{1}}}^{1}} W^{\vec{\lambda}, \vec{x}, \varepsilon}\left(B_{s}\right) d s\right)\right) \\
& +E_{x}\left(1_{\left\{T_{2 \varepsilon}^{2}<\infty\right\}} 1_{\left\{T_{2 \varepsilon}^{2}<T_{r_{\lambda_{1}}}^{1}\right\}} \lambda_{1}^{1+\alpha} W_{1}^{\vec{\lambda}, \vec{x}, \varepsilon}\left(B\left(T_{2 \varepsilon}^{2}\right)\right) \exp \left(-\int_{0}^{T_{2 \varepsilon}^{2}} W^{\vec{\lambda}, \vec{x}, \varepsilon}\left(B_{s}\right) d s\right)\right) \\
:= & I_{1}+I_{2} .
\end{aligned}
$$


We first deal with $I_{2}$. Note in the integrand of $I_{2}$ we may assume that $\left|B\left(T_{2 \varepsilon}^{2}\right)-x_{2}\right|=2 \varepsilon$ and so for $\varepsilon<\left|x_{1}-x_{2}\right| / 4$ we have $\left|x_{1}-B\left(T_{2 \varepsilon}^{2}\right)\right|>\Delta / 2$ where $\Delta=\left|x_{1}-x_{2}\right|$. Apply (5.4) with $x=B\left(T_{2 \varepsilon}^{2}\right)$ to get

$$
\lambda_{1}^{1+\alpha} W_{1}^{\vec{\lambda}, \vec{x}, \varepsilon}\left(B\left(T_{2 \varepsilon}^{2}\right)\right) \leq c_{3.2}\left|B\left(T_{2 \varepsilon}^{2}\right)-x_{1}\right|^{-p} \leq c_{3.2} \Delta^{-p} 2^{p} .
$$

Let $\tau_{r}=\inf \left\{t \geq 0:\left|B_{t}\right| \leq r\right\}$ and use (C.28) and (8.8) to see that $I_{2}$ becomes

$$
\begin{aligned}
I_{2} & \leq c_{3.2} 2^{p} \Delta^{-p} E_{x}\left(1_{\left\{T_{2 \varepsilon}^{2}<\infty\right\}} 1_{\left\{T_{2 \varepsilon}^{2}<T_{r_{\lambda_{1}}}^{1}\right\}} \exp \left(-\int_{0}^{T_{2 \varepsilon}^{2}} W^{\vec{\lambda}, \vec{x}, \varepsilon}\left(B_{s}\right) d s\right)\right) \\
& \leq c_{3.2} 2^{p} \Delta^{-p} E_{x-x_{2}}\left(1_{\left\{\tau_{2 \varepsilon}<\infty\right\}} \exp \left(-\int_{0}^{\tau_{2 \varepsilon}} V^{\infty}\left(B_{s}\right) d s\right)\right) \\
& =c_{3.2} 2^{p} \Delta^{-p}\left(2 \varepsilon /\left|x-x_{2}\right|\right)^{p} \rightarrow 0 \text { as } \varepsilon \downarrow 0,
\end{aligned}
$$

where we have used Proposition 3.7 in the last equality with $g=V^{\infty}$.

Now we will turn to $I_{1}$. Let $\left(Y_{t}, t \geq 0\right)$ be the $d$-dimensional coordinate process under Wiener measure, $P_{x}$. By slightly abusing the notation, we set $\tau_{r}=\tau_{r}^{Y}=\inf \left\{t \geq 0:\left|Y_{t}\right| \leq\right.$ $r$ for any $r>0$, and set

$$
T_{2 \varepsilon}^{\prime}=T_{2 \varepsilon}^{\prime, Y}=\inf \left\{t \geq 0:\left|Y_{t}-\left(x_{2}-x_{1}\right)\right| \leq 2 \varepsilon\right\} .
$$

Then use translation invariance of $Y$ to get

$$
\begin{aligned}
I_{1}= & E_{x-x_{1}}\left(1_{\left\{\tau_{\left.r_{\lambda_{1}}<\infty\right\}} 1_{\left\{\tau_{\lambda_{\lambda_{1}}}<T_{2 \varepsilon}^{\prime}\right\}} \lambda_{1}^{1+\alpha} W_{1}^{\vec{\lambda}, \vec{x}, \varepsilon}\left(Y\left(\tau_{r_{\lambda_{1}}}\right)+x_{1}\right)\right.}\right. \\
& \left.\times \exp \left(-\int_{0}^{\tau_{r_{\lambda_{1}}}} W^{\vec{\lambda}, \vec{x}, \varepsilon}\left(Y_{s}+x_{1}\right) d s\right)\right) .
\end{aligned}
$$

Recall that $\hat{P}_{x}^{(2-2 \nu)}$ is the law of $Y$ starting from $x$ such that $Y$ satisfy the SDE as in (C.5). Now apply Proposition 7.1 with $g(\cdot)=W^{\vec{\lambda}, \vec{x}, \varepsilon}\left(\cdot+x_{1}\right)$ to get

$$
\begin{aligned}
I_{1}=\frac{r_{\lambda_{1}}^{p}}{\left|x-x_{1}\right|^{p}} \hat{E}_{x-x_{1}}^{(2-2 \nu)}\left(1_{\left\{\tau_{r_{\lambda_{1}}}<T_{2 \varepsilon}^{\prime}\right\}} \lambda_{1}^{1+\alpha} W_{1}^{\vec{\lambda}, \vec{x}, \varepsilon}\left(Y\left(\tau_{r_{\lambda_{1}}}\right)+x_{1}\right)\right. \\
\left.\times \exp \left(-\int_{0}^{\tau_{r_{\lambda_{1}}}}\left(W^{\vec{\lambda}, \vec{x}, \varepsilon}\left(Y_{s}+x_{1}\right)-V^{\infty}\left(Y_{s}\right)\right) d s\right)\right) \\
=\frac{1}{\left|x-x_{1}\right|^{p}} \hat{E}_{x-x_{1}}^{(2-2 \nu)}\left(\left[1_{\left\{\tau_{r_{\lambda_{1}}}<T_{2 \varepsilon}^{\prime}\right\}}\right]\left[r_{\lambda_{1}}^{p} \lambda_{1}^{1+\alpha} W_{1}^{\vec{\lambda}, \vec{x}, \varepsilon}\left(Y\left(\tau_{r_{\lambda_{1}}}\right)+x_{1}\right)\right]\right. \\
\times\left[\exp \left(-\int_{0}^{\tau_{r_{\lambda_{1}}}}\left(W^{\vec{\lambda}, \vec{x}, \varepsilon}\left(Y_{s}+x_{1}\right)-V^{\lambda_{1}}\left(Y_{s}\right)\right) d s\right)\right] \\
\left.\times\left[\exp \left(\int_{0}^{\tau_{r_{\lambda_{1}}}}\left(V^{\infty}\left(Y_{s}\right)-V^{\lambda_{1}}\left(Y_{s}\right)\right) d s\right)\right]\right) \\
:=\frac{1}{\left|x-x_{1}\right|^{p}} \hat{E}_{x-x_{1}}^{(2-2 \nu)}\left(\left[\hat{J}_{1}\right]\left[\hat{J}_{2}\right]\left[\hat{J}_{3}\right]\left[\hat{J}_{4}\right]\right) .
\end{aligned}
$$

We first consider $\hat{J}_{2}$. Recall the definition of $W_{1}^{\vec{\lambda}, \vec{x}, \varepsilon}$ as in Section 4 and use translation invariance to get

$$
\begin{aligned}
\hat{J}_{2} & =r_{\lambda_{1}}^{p} \lambda_{1}^{1+\alpha} \mathbb{N}_{Y\left(\tau_{r_{\lambda_{1}}}\right)+x_{1}}\left(L^{x_{1}} e^{-\lambda_{1} L^{x_{1}}} \exp \left(-\lambda_{2} \frac{X_{G_{\varepsilon}^{x_{2}}}(1)}{\varepsilon^{2}}\right) 1_{\left\{X_{G_{\varepsilon / 2}^{x_{2}}}=0\right\}}\right) \\
& =r_{\lambda_{1}}^{p} \lambda_{1}^{1+\alpha} \mathbb{N}_{Y\left(\tau_{r_{\lambda_{1}}}\right)}\left(L^{0} e^{-\lambda_{1} L^{0}} \exp \left(-\lambda_{2} \frac{X_{G_{\varepsilon}^{x_{2}-x_{1}}(1)}}{\varepsilon^{2}}\right) 1_{\left\{X_{G_{\varepsilon / 2}^{x_{2}-x_{1}}}=0\right\}}\right) .
\end{aligned}
$$


By the scaling of Brownian snake and its local time and exit measure under the excursion measure $\mathbb{N}_{x}$ (see, e.g., Proof of Proposition V.9 in [16]), we have

$$
\begin{aligned}
\hat{J}_{2}=r_{\lambda_{1}}^{p} \lambda_{1}^{1+\alpha} r_{\lambda_{1}}^{-2} \mathbb{N}_{Y\left(\tau_{r_{\lambda_{1}}}\right) / r_{\lambda_{1}}}\left(r_{\lambda_{1}}^{4-d} L^{0} \exp \left(-\lambda_{1} r_{\lambda_{1}}^{4-d} L^{0}\right)\right. & \\
& \left.\times \exp \left(-\lambda_{2} \frac{X_{G_{\varepsilon / r_{\lambda_{1}}}^{\left(x_{2}-x_{1}\right) / r_{\lambda_{1}}}}(1)}{\left(\varepsilon / r_{\lambda_{1}}\right)^{2}}\right) 1\left(X_{G_{\varepsilon / 2 r_{\lambda_{1}}}^{\left(x_{2}-x_{1}\right) / r_{\lambda_{1}}}}=0\right)\right) .
\end{aligned}
$$

Use the definitions of $r_{\lambda_{1}}$ and $\alpha$ to see that the above becomes

$$
\begin{aligned}
& \hat{J}_{2}=\lambda_{0}^{p+2-d} \mathbb{N}_{Y\left(\tau_{r_{1}}\right) / r_{\lambda_{1}}}\left(L^{0} \exp \left(-\lambda_{0}^{4-d} L^{0}\right)\right. \\
& \quad\left.\exp \left(-\lambda_{2} \frac{X_{G_{\varepsilon / r_{\lambda_{1}}}^{\left(x_{2}-x_{1}\right) / r_{\lambda_{1}}}}(1)}{\left(\varepsilon / r_{\lambda_{1}}\right)^{2}}\right) 1\left(X_{G_{\varepsilon / 2 r_{\lambda_{1}}}^{\left(x_{2}-x_{1}\right) / r_{\lambda_{1}}}}=0\right)\right) \\
& \stackrel{\text { law }}{=} \lambda_{0}^{p+2-d} \mathbb{N}_{Y\left(\tau_{1}\right)}\left(L^{0} \exp \left(-\lambda_{0}^{4-d} L^{0}\right)\right. \\
&\left.\quad \times \exp \left(-\lambda_{2} \frac{X_{G_{\varepsilon / r_{\lambda_{1}}}^{\left(x_{2}-x_{1}\right) / r_{\lambda_{1}}}}(1)}{\left(\varepsilon / r_{\lambda_{1}}\right)^{2}}\right) 1\left(X_{G_{\varepsilon / 2 r_{\lambda_{1}}}^{\left(x_{2}-x_{1}\right) / r_{\lambda_{1}}}}=0\right)\right),
\end{aligned}
$$

where the last equality follows from the scaling of $Y$. Note for any $K>0$, for all $0<\varepsilon<\left|x_{1}-x_{2}\right| / 2$, we have

$$
\left|\frac{x_{2}-x_{1}}{r_{\lambda_{1}}}\right|-\frac{\varepsilon}{r_{\lambda_{1}}} \geq \frac{\left|x_{1}-x_{2}\right| / 2}{r_{\lambda_{1}}}>K \text { for } \lambda_{1} \text { large enough, }
$$

and so by (2.3) and (2.4) we conclude $\mathbb{N}_{Y\left(\tau_{1}\right)}$-a.e.

$$
X_{G_{\varepsilon / r_{\lambda_{1}}}^{\left(x_{2}-x_{1}\right) / r_{\lambda_{1}}}}(1)=X_{G_{\varepsilon / 2 r_{\lambda_{1}}}^{\left(x_{2}-x_{1}\right) / r_{\lambda_{1}}}}(1)=0 \text { for } \lambda_{1} \text { large enough. }
$$

Therefore an application of the dominated convergence theorem Theorem will give us

$$
\begin{gathered}
\lim _{\lambda_{1} \rightarrow \infty, \varepsilon \downarrow 0} \mathbb{N}_{Y\left(\tau_{1}\right)}\left(L^{0} e^{-\lambda_{0}^{4-d} L^{0}} \exp \left(-\lambda_{2} \frac{X_{G_{\varepsilon / r_{\lambda_{1}}}^{\left(x_{2}-x_{1}\right) / r_{\lambda_{1}}}}(1)}{\left(\varepsilon / r_{\lambda_{1}}\right)^{2}}\right) 1\left(X_{G_{\varepsilon / 2 r_{\lambda_{1}}}^{\left(x_{2}-x_{1}\right) / r_{\lambda_{1}}}}=0\right)\right) \\
=\mathbb{N}_{Y\left(\tau_{1}\right)}\left(L^{0} e^{-\lambda_{0}^{4-d} L^{0}}\right)=\mathbb{N}_{e_{1}}\left(L^{0} e^{-\lambda_{0}^{4-d} L^{0}}\right)=V_{1}^{\lambda_{0}^{4-d}}(1)
\end{gathered}
$$

where in the next to last equality we have used spherical symmetry and $e_{1}$ is the first unit basis vector. The last equality follows by (3.2). In view of (C.32), we have proved

$$
\hat{J}_{2}=r_{\lambda_{1}}^{p} \lambda_{1}^{1+\alpha} W_{1}^{\vec{\lambda}, \vec{x}, \varepsilon}\left(Y_{\tau_{r_{\lambda_{1}}}}+x_{1}\right) \rightarrow \lambda_{0}^{p+2-d} V_{1}^{\lambda_{0}^{4-d}}(1) \text { in distribution }
$$

as $\lambda_{1} \rightarrow \infty, \varepsilon \downarrow 0$, and furthermore under $\hat{P}_{x-x_{1}}^{(2-2 \nu)}$, we have

$$
\hat{J}_{2}=r_{\lambda_{1}}^{p} \lambda_{1}^{1+\alpha} W_{1}^{\vec{\lambda}, \vec{x}, \varepsilon}\left(Y_{\tau_{r_{\lambda_{1}}}}+x_{1}\right) \rightarrow \lambda_{0}^{p+2-d} V_{1}^{\lambda_{0}^{4-d}}(1) \text { in probability }
$$

as $\lambda_{1} \rightarrow \infty, \varepsilon \downarrow 0$ since $\lambda_{0}^{p+2-d} V_{1}^{\lambda_{0}^{4-d}}(1)$ is a constant.

By (8.20), with $\hat{P}_{x-x_{1}}^{(2-2 \nu)}$-probability one we have

$$
\hat{J}_{1}=1_{\left\{\tau_{r_{\lambda_{1}}}<T_{2 \varepsilon}^{\prime}\right\}} \rightarrow 1 \text { as } \lambda_{1} \rightarrow \infty, \varepsilon \downarrow 0 .
$$


As for (8.21), we use Lemma 8.1 to see that with $\hat{P}_{x-x_{1}}^{(2-2 \nu)}$-probability one,

$$
\begin{aligned}
\hat{J}_{3}=\exp (- & \left.\int_{0}^{\tau_{r_{\lambda_{1}}}}\left(W^{\vec{\lambda}, \vec{x}, \varepsilon}\left(Y_{s}+x_{1}\right)-V^{\lambda_{1}}\left(Y_{s}\right)\right) d s\right) \\
& \rightarrow \exp \left(-\int_{0}^{\tau_{0}}\left(V^{\vec{\infty}, \vec{x}}\left(Y_{s}+x_{1}\right)-V^{\infty}\left(Y_{s}\right)\right) d s\right) \text { as } \lambda_{1} \rightarrow \infty, \varepsilon \downarrow 0 .
\end{aligned}
$$

Here one can see from (8.5) that

$$
0 \leq W^{\vec{\lambda}, \vec{x}, \varepsilon}\left(Y_{s}+x_{1}\right)-V^{\lambda_{1}}\left(Y_{s}\right) \leq U^{\widetilde{\lambda}_{2} \varepsilon^{-2}, \varepsilon}\left(Y_{s}-\left(x_{2}-x_{1}\right)\right),
$$

where $\widetilde{\lambda}_{2}$ is as in (8.2). Then argue as in the derivation of (8.27) and apply the dominated convergence theorem as before.

Combine (C.34), (C.35) and (C.36) to see that under $\hat{P}_{x-x_{1}}^{(2-2 \nu)}$, we have

$$
\begin{gathered}
\hat{J}_{1} \hat{J}_{2} \hat{J}_{3} \rightarrow \lambda_{0}^{p+2-d} V_{1}^{\lambda_{0}^{4-d}}(1) \exp \left(-\int_{0}^{\tau_{0}}\left(V^{\vec{\infty}, \vec{x}}\left(Y_{s}+x_{1}\right)-V^{\infty}\left(Y_{s}\right)\right) d s\right) \\
\text { in probability as } \lambda_{1} \rightarrow \infty, \varepsilon \downarrow 0 .
\end{gathered}
$$

Recall from (5.4) to see that

$$
\widetilde{J_{2}} \leq r_{\lambda_{1}}^{p} \lambda_{1}^{1+\alpha} W_{1}^{\vec{\lambda}, \vec{x}, \varepsilon}\left(Y\left(\tau_{r_{\lambda_{1}}}\right)+x_{1}\right) \leq r_{\lambda_{1}}^{p} c_{3.2}\left|Y\left(\tau_{r_{\lambda_{1}}}\right)\right|^{-p}=c_{3.2} .
$$

By (C.37) we have $\hat{J}_{3} \leq 1$ and so conclude

$$
\hat{J}_{1} \hat{J}_{2} \hat{J}_{3} \leq c_{3.2}, \hat{P}_{x-x_{1}}^{(2-2 \nu)} \text {-a.s. }
$$

Recall (8.32) and use (C.38), (C.39) and the bounded convergence theorem to get

$$
\begin{aligned}
\lim _{\lambda_{1} \rightarrow \infty, \varepsilon \downarrow 0} \hat{E}_{x-x_{1}}^{(2-2 \nu)} & \left(\left(\lambda_{0}^{p+2-d} V_{1}^{\lambda_{0}^{4-d}}(1) \exp \left(-\int_{0}^{\tau_{0}}\left(V^{\vec{\infty}, \vec{x}}\left(Y_{s}+x_{1}\right)-V^{\infty}\left(Y_{s}\right)\right) d s\right)\right.\right. \\
& \left.\left.-\hat{J}_{1} \hat{J}_{2} \hat{J}_{3}\right)^{2}\right)=0 .
\end{aligned}
$$

Recall $\widetilde{J_{4}}$ from (C.6) to see that

$$
\hat{J}_{4}=\exp \left(\int_{0}^{\tau_{r_{\lambda_{1}}}}\left(V^{\infty}\left(Y_{s}\right)-V^{\lambda_{1}}\left(Y_{s}\right)\right) d s\right)=\widetilde{J_{4}} .
$$

By (C.16) and by choosing $\lambda_{0}>c_{3.5}$, we have

$$
\hat{E}_{x-x_{1}}^{(2-2 \nu)}\left(\hat{J}_{4}^{2}\right)=\hat{E}_{x-x_{1}}^{(2-2 \nu)}\left({\widetilde{J_{4}}}^{2}\right) \leq C_{3.5}\left(\lambda_{0}, \nu, 2\right)<\infty, \forall \lambda_{1}>0 .
$$

Now we conclude

$$
\begin{aligned}
& \mid \hat{E}_{x-x_{1}}^{(2-2 \nu)}\left(\hat{J}_{1} \hat{J}_{2} \hat{J}_{3} \hat{J}_{4}\right)-\hat{E}_{x-x_{1}}^{(2-2 \nu)}\left(\lambda_{0}^{p+2-d} V_{1}^{\lambda_{0}^{4-d}}(1)\right. \\
& \left.\exp \left(-\int_{0}^{\tau_{0}}\left(V^{\vec{\infty}, \vec{x}}\left(Y_{s}+x_{1}\right)-V^{\infty}\left(Y_{s}\right)\right) d s\right) \cdot \hat{J}_{4}\right) \mid \\
\leq & \hat{E}_{\left|x-x_{1}\right|}^{(2-2 \nu)}\left(\hat{J}_{4} \cdot \mid \hat{J}_{1} \hat{J}_{2} \hat{J}_{3}-\lambda_{0}^{p+2-d} V_{1}^{\lambda_{0}^{4-d}}(1)\right. \\
& \left.\exp \left(-\int_{0}^{\tau_{0}}\left(V^{\vec{\infty}, \vec{x}}\left(Y_{s}+x_{1}\right)-V^{\infty}\left(Y_{s}\right)\right) d s\right) \mid\right) \\
\leq & \hat{E}_{x-x_{1}}^{(2-2 \nu)}\left(\left(\lambda_{0}^{p+2-d} V_{1}^{\lambda_{0}^{4-d}}(1) \exp \left(-\int_{0}^{\tau_{0}}\left(V^{\vec{\infty}, \vec{x}}\left(Y_{s}+x_{1}\right)-V^{\infty}\left(Y_{s}\right)\right) d s\right)\right.\right. \\
& \left.\left.-\hat{J}_{1} \hat{J}_{2} \hat{J}_{3}\right)^{2}\right)^{1 / 2}\left(\hat{E}_{x-x_{1}}^{(2-2 \nu)}\left(\hat{J}_{4}^{2}\right)\right)^{1 / 2} \rightarrow 0 \text { as } \lambda_{1} \rightarrow \infty, \varepsilon \downarrow 0,
\end{aligned}
$$


where the second inequality is by Cauchy-Schwartz and the convergence to 0 follows from (C.40) and (C.41). In view of (C.31), we have

$$
\begin{aligned}
& \lim _{\lambda_{1} \rightarrow \infty, \varepsilon \downarrow 0} I_{1} \\
& =\frac{\lambda_{0}^{p+2-d} V_{1}^{\lambda_{0}^{4-d}}(1)}{\left|x-x_{1}\right|^{p}} \lim _{\lambda_{1} \rightarrow \infty} \hat{E}_{x-x_{1}}^{(2-2 \nu)}\left(\exp \left(-\int_{0}^{\tau_{0}}\left(V^{\vec{\infty}, \vec{x}}\left(Y_{s}+x_{1}\right)-V^{\infty}\left(Y_{s}\right)\right) d s\right) \cdot \hat{J}_{4}\right) \\
& =\frac{\lambda_{0}^{p+2-d} V_{1}^{\lambda_{0}^{4-d}}(1)}{\left|x-x_{1}\right|^{p}} C_{3.5}\left(\lambda_{0}, \nu, 1\right) \hat{E}_{x-x_{1}}^{(2-2 \nu)}\left(\exp \left(-\int_{0}^{\tau_{0}}\left(V^{\vec{\infty}, \vec{x}}\left(Y_{s}+x_{1}\right)-V^{\infty}\left(Y_{s}\right)\right) d s\right)\right),
\end{aligned}
$$

where the last equality is by (C.18) (recall $\hat{J}_{4}=\widetilde{J_{4}}$ ).

Now we conclude from (C.27), (C.29) and (C.42) that

$$
\begin{gathered}
\lim _{\lambda_{1} \rightarrow \infty, \varepsilon \downarrow 0} \lambda_{1}^{1+\alpha} W_{1}^{\vec{\lambda}, \vec{x}, \varepsilon}(x)=\lambda_{0}^{p+2-d} V_{1}^{\lambda_{0}^{4-d}}(1) C_{3.5}\left(\lambda_{0}, \nu, 1\right)\left|x-x_{1}\right|^{-p} \\
\hat{E}_{x-x_{1}}^{(2-2 \nu)}\left(\exp \left(-\int_{0}^{\tau_{0}}\left(V^{\vec{\infty}, \vec{x}}\left(Y_{s}+x_{1}\right)-V^{\infty}\left(Y_{s}\right)\right) d s\right)\right)
\end{gathered}
$$

and the proof is complete.

\section{Proofs of Proposition 4.2(i) and Proposition 4.3(ii)}

Proof of Proposition 4.2(i). For any $x_{1} \neq x_{2}$, we fix $x \neq x_{1}, x_{2}$. In order to find the limit of $\lambda_{1}^{1+\alpha} \lambda_{2}^{1+\alpha}\left(-V_{1,2}^{\vec{\lambda}, \vec{x}}(x)\right)$ as $\lambda_{1}, \lambda_{2} \rightarrow \infty$, by Lemma 9.2 , it suffices to find the limits of the following as $\lambda_{1}, \lambda_{2} \rightarrow \infty$.

$$
\begin{aligned}
K_{1}+ & K_{2} \equiv \lambda_{1}^{1+\alpha} \lambda_{2}^{1+\alpha} E_{x}\left(\int_{0}^{T_{r_{\lambda}}} \prod_{i=1}^{2} V_{i}^{\vec{\lambda}, \vec{x}}\left(B_{t}\right) \exp \left(-\int_{0}^{t} V^{\vec{\lambda}, \vec{x}}\left(B_{s}\right) d s\right) d t\right) \\
+ & \lambda_{1}^{1+\alpha} \lambda_{2}^{1+\alpha} E_{x}\left(\exp \left(-\int_{0}^{T_{r_{\lambda}}} V^{\vec{\lambda}, \vec{x}}\left(B_{s}\right) d s\right) 1_{\left(T_{r_{\lambda}}<\infty\right)}\left(-V_{1,2}^{\vec{\lambda}, \vec{x}}\left(B_{T_{r_{\lambda}}}\right)\right)\right) .
\end{aligned}
$$

In the above $T_{r_{\lambda}}=T_{r_{\lambda_{1}}}^{1} \wedge T_{r_{\lambda_{2}}}^{2}$ and $T_{r_{\lambda_{i}}}^{i}=\inf \left\{t \geq 0:\left|B_{t}-x_{i}\right| \leq r_{\lambda_{i}}\right\}$. Here $r_{\lambda_{i}}=\lambda_{0} \lambda_{i}^{-\frac{1}{4-d}}$ and we will choose $\lambda_{0}$ to be some fixed large constant below. Let $\lambda_{1}, \lambda_{2}>0$ be large so that

$$
0<4\left(r_{\lambda_{1}} \vee r_{\lambda_{2}}\right)<\min \left\{\left|x_{1}-x\right|,\left|x_{2}-x\right|,\left|x_{1}-x_{2}\right|\right\}
$$

We first consider $K_{2}$. On $\left\{T_{r_{\lambda}}<\infty\right\}$, by considering $T_{r_{\lambda}}=T_{r_{\lambda_{i}}}^{i}<T_{r_{\lambda_{3} i}}^{3-i}$ we may set $x_{\lambda}(\omega)=B\left(T_{r_{\lambda}}\right)=B\left(T_{r_{\lambda_{i}}}^{i}\right)$ so that $\left|x_{\lambda}-x_{i}\right|=r_{\lambda_{i}}$ and by (D.2) we have $\left|x_{3-i}-x_{\lambda}\right| \geq \Delta / 2$ where $\Delta=\left|x_{1}-x_{2}\right|$. Lemma 9.1 and the above imply

$$
\left(-V_{1,2}^{\vec{\lambda}, \vec{x}, \vec{\varepsilon}}\left(B\left(T_{r_{\lambda}}\right)\right)\right) \leq \frac{2}{\lambda_{i}} c_{3.2} \lambda_{3-i}^{-(1+\alpha)} \Delta^{-p} 2^{p} \leq c \Delta^{-p} \frac{1}{\lambda_{3-i}^{1+\alpha}} \frac{1}{\lambda_{i}} .
$$

This shows that

$$
\begin{aligned}
K_{2} \leq \lambda_{1}^{1+\alpha} \lambda_{2}^{1+\alpha} & \sum_{i=1}^{2} c \Delta^{-p} \frac{1}{\lambda_{3-i}^{1+\alpha}} \frac{1}{\lambda_{i}} \\
& E_{x}\left(1\left(T_{r_{\lambda_{i}}}^{i}<\infty\right) 1\left(T_{r_{\lambda_{i}}}^{i}<T_{r_{\lambda_{3} i}}^{3-i}\right) \exp \left(-\int_{0}^{T_{r_{\lambda_{i}}}^{i}} V^{\vec{\lambda}, \vec{x}}\left(B_{s}\right) d s\right)\right) .
\end{aligned}
$$


From (C.3), by choosing $\lambda_{0}>c_{3.5}$ we have for $i=1,2$,

$$
\begin{aligned}
& E_{x}\left(1\left(T_{r_{\lambda_{i}}}^{i}<\infty\right) 1\left(T_{r_{\lambda_{i}}}^{i}<T_{r_{\lambda_{3} i}}^{3-i}\right) \exp \left(-\int_{0}^{T_{r_{\lambda_{i}}}^{i}} V^{\vec{\lambda}, \vec{x}}\left(B_{s}\right) d s\right)\right) \\
& \leq r_{\lambda_{i}}^{p}\left|x-x_{i}\right|^{-p} C_{3.5}\left(\lambda_{0}, \nu, 1\right),
\end{aligned}
$$

and so (D.3) becomes

$$
\begin{aligned}
K_{2} & \leq \lambda_{1}^{1+\alpha} \lambda_{2}^{1+\alpha} c \Delta^{-p} \sum_{i=1}^{2} \frac{1}{\lambda_{3-i}^{1+\alpha}} \frac{1}{\lambda_{i}} r_{\lambda_{i}}^{p}\left|x-x_{i}\right|^{-p} C_{3.5}\left(\lambda_{0}, \nu, 1\right) \\
& \leq C \Delta^{-p} \lambda_{0}^{p}\left(\lambda_{1}^{-\frac{2}{4-d}}+\lambda_{2}^{-\frac{2}{4-d}}\right) \sum_{i=1}^{2}\left|x-x_{i}\right|^{-p} \rightarrow 0 \text { as } \lambda_{1}, \lambda_{2} \rightarrow \infty
\end{aligned}
$$

where in the last equality we have used the definitions of $r_{\lambda_{i}}$ and $\alpha$.

Now we will turn to $K_{1}$. Recall

$$
K_{1}=\iint_{0}^{\infty} \lambda_{1}^{1+\alpha} \lambda_{2}^{1+\alpha} V_{1}^{\vec{\lambda}, \vec{x}}\left(B_{t}\right) V_{2}^{\vec{\lambda}, \vec{x}}\left(B_{t}\right) \exp \left(-\int_{0}^{t} V^{\vec{\lambda}, \vec{x}}\left(B_{s}\right) d s\right) 1\left(t \leq T_{r_{\lambda}}\right) d t d P_{x} .
$$

By Proposition 4.1 and Lemma 8.1, for $\operatorname{Leg} \times P_{x}$-a.e. $(t, \omega)$, we have

$$
\begin{gathered}
\lim _{\lambda_{1}, \lambda_{2} \rightarrow \infty} \lambda_{1}^{1+\alpha} \lambda_{2}^{1+\alpha} V_{1}^{\vec{\lambda}, \vec{x}}\left(B_{t}\right) V_{2}^{\vec{\lambda}, \vec{x}}\left(B_{t}\right) \exp \left(-\int_{0}^{t} V^{\vec{\lambda}, \vec{x}}\left(B_{s}\right) d s\right) 1\left(t \leq T_{r_{\lambda}}\right) \\
=K_{4.1}^{2} U_{1}^{\vec{\infty}, \vec{x}}\left(B_{t}\right) U_{2}^{\vec{\infty}, \vec{x}}\left(B_{t}\right) \exp \left(-\int_{0}^{t} V^{\vec{\infty}, \vec{x}}\left(B_{s}\right) d s\right) .
\end{gathered}
$$

Use the bounds (5.2) and (8.4) to see that

$$
\begin{aligned}
& \lambda_{1}^{1+\alpha} \lambda_{2}^{1+\alpha} V_{1}^{\vec{\lambda}, \vec{x}}\left(B_{t}\right) V_{2}^{\vec{\lambda}, \vec{x}}\left(B_{t}\right) \exp \left(-\int_{0}^{t} V^{\vec{\lambda}, \vec{x}}\left(B_{s}\right) d s\right) 1\left(t \leq T_{r_{\lambda}}\right) \\
\leq & c_{3.2}^{2}\left|B_{t}-x_{1}\right|^{-p}\left|B_{t}-x_{2}\right|^{-p} \exp \left(-\int_{0}^{t} V^{\vec{\lambda}, \vec{x}}\left(B_{s}\right) d s\right) 1\left(t \leq T_{r_{\lambda}}\right) \\
\leq & c_{3.2}^{2} \sum_{i=1}^{2}\left|B_{t}-x_{1}\right|^{-p}\left|B_{t}-x_{2}\right|^{-p} 1\left(\left|B_{t}-x_{i}\right| \leq\left|B_{t}-x_{3-i}\right|\right) \\
& \exp \left(-\int_{0}^{t} V^{\lambda_{i}}\left(B_{s}-x_{i}\right) d s\right) 1\left(t \leq T_{r_{\lambda}}\right) \\
\leq & c_{3.2}^{2} 2^{p} \Delta^{-p} \sum_{i=1}^{2}\left|B_{t}-x_{i}\right|^{-p} \exp \left(-\int_{0}^{t} V^{\lambda_{i}}\left(B_{s}-x_{i}\right) d s\right) 1\left(t \leq T_{r_{\lambda_{i}}}^{i}\right),
\end{aligned}
$$

where we have used $\left|B_{t}-x_{3-i}\right|>\Delta / 2$ on $\left\{\left|B_{t}-x_{i}\right| \leq\left|B_{t}-x_{3-i}\right|\right\}$ and $T_{r_{\lambda}} \leq T_{r_{\lambda_{i}}}^{i}$ in the last inequality. It is clear that for $\operatorname{Leg} \times P_{x}$-a.e. $(t, \omega)$ we have

$$
\begin{aligned}
& \lim _{\lambda_{i} \rightarrow \infty}\left|B_{t}-x_{i}\right|^{-p} \exp \left(-\int_{0}^{t} V^{\lambda_{i}}\left(B_{s}-x_{i}\right) d s\right) 1\left(t \leq T_{r_{\lambda_{i}}}^{i}\right) \\
& =\left|B_{t}-x_{i}\right|^{-p} \exp \left(-\int_{0}^{t} V^{\infty}\left(B_{s}-x_{i}\right) d s\right) .
\end{aligned}
$$

In view of (D.6), (D.7) and (D.8), if one can show that for $i=1,2$,

$$
\begin{gathered}
\lim _{\lambda_{i} \rightarrow \infty} \iint_{0}^{\infty}\left|B_{t}-x_{i}\right|^{-p} \exp \left(-\int_{0}^{t} V^{\lambda_{i}}\left(B_{s}-x_{i}\right) d s\right) 1\left(t \leq T_{r_{\lambda_{i}}}^{i}\right) d t d P_{x} \\
=\iint_{0}^{\infty}\left|B_{t}-x_{i}\right|^{-p} \exp \left(-\int_{0}^{t} V^{\infty}\left(B_{s}-x_{i}\right) d s\right) d t d P_{x}<\infty,
\end{gathered}
$$


then a generalized the dominated convergence theorem Theorem (see, e.g., Exercise 20 of Chp. 2 of [4]) implies that

$$
\begin{aligned}
\lim _{\lambda_{1}, \lambda_{2} \rightarrow \infty} K_{1}= & \lim _{\lambda_{1}, \lambda_{2} \rightarrow \infty} \iint_{0}^{\infty} \lambda_{1}^{1+\alpha} \lambda_{2}^{1+\alpha} V_{1}^{\vec{\lambda}, \vec{x}}\left(B_{t}\right) V_{2}^{\vec{\lambda}, \vec{x}}\left(B_{t}\right) \\
& \quad \exp \left(-\int_{0}^{t} V^{\vec{\lambda}, \vec{x}}\left(B_{s}\right) d s\right) 1\left(t \leq T_{r_{\lambda}}\right) d t d P_{x} \\
= & K_{4.1}^{2} \iint_{0}^{\infty} U_{1}^{\vec{\infty}, \vec{x}}\left(B_{t}\right) U_{2}^{\vec{\infty}, \vec{x}}\left(B_{t}\right) \exp \left(-\int_{0}^{t} V^{\vec{\infty}, \vec{x}}\left(B_{s}\right) d s\right) d t d P_{x} \\
= & K_{4.1}^{2}\left(-U_{1,2}^{\vec{\infty}, \vec{x}}(x)\right)
\end{aligned}
$$

where the last is by (1.17). The proof will then be finished by Lemma 9.2, (D.1), (D.5) and (D.10).

It remains to prove (D.9) and it suffices to consider $i=1$. We first show that for any $0<q<6-p$, we have

$$
\sup _{\lambda>0} \iint_{0}^{\infty}\left|B_{t}-x_{1}\right|^{-q} \exp \left(-\int_{0}^{t} V^{\lambda}\left(B_{s}-x_{1}\right) d s\right) 1\left(t \leq T_{r_{\lambda}}^{1}\right) d t d P_{x}<\infty .
$$

Assuming the above, we can apply Fatou's Lemma to see that

$$
\begin{aligned}
& \iint_{0}^{\infty}\left|B_{t}-x_{1}\right|^{-q} \exp \left(-\int_{0}^{t} V^{\infty}\left(B_{s}-x_{1}\right) d s\right) d t d P_{x} \\
& \leq \liminf _{\lambda \rightarrow \infty} \iint_{0}^{\infty}\left|B_{t}-x_{1}\right|^{-q} \exp \left(-\int_{0}^{t} V^{\lambda}\left(B_{s}-x_{1}\right) d s\right) 1\left(t \leq T_{r_{\lambda}}^{1}\right) d t d P_{x}<\infty,
\end{aligned}
$$

thus giving the finiteness in (D.9) (recall $p \in(2,3)$ ).

To see that (D.11) holds, by Fubini's theorem and translation invariance we have

$$
\begin{aligned}
I(\lambda) & :=\iint_{0}^{\infty}\left|B_{t}-x_{1}\right|^{-q} \exp \left(-\int_{0}^{t} V^{\lambda}\left(B_{s}-x_{1}\right) d s\right) 1\left(t \leq T_{r_{\lambda}}^{1}\right) d t d P_{x} \\
& =\int_{0}^{\infty} E_{x-x_{1}}\left(\left|B_{t}\right|^{-q} \exp \left(-\int_{0}^{t} V^{\lambda}\left(B_{s}\right) d s\right) 1\left(t \leq \tau_{r_{\lambda}}\right)\right) d t,
\end{aligned}
$$

where $\tau_{r}=\inf \left\{t \geq 0:\left|B_{t}\right| \leq r\right\}$ for any $r>0$. Let $\mu, \nu$ are as in (1.13) and then apply Lemma 3.6 to get

$$
\begin{aligned}
& E_{x-x_{1}}\left(\left|B_{t}\right|^{-q} \exp \left(-\int_{0}^{t} V^{\lambda}\left(B_{s}\right) d s\right) 1\left(t \leq \tau_{r_{\lambda}}\right)\right) \\
= & E_{\left|x-x_{1}\right|}^{(2+2 \mu)}\left(\rho_{t}^{-q} \exp \left(-\int_{0}^{t} V^{\lambda}\left(\rho_{s}\right) d s\right) 1\left(t \leq \tau_{r_{\lambda}}\right)\right) \\
= & \left|x-x_{1}\right|^{\nu-\mu} E_{\left|x-x_{1}\right|}^{(2+2 \nu)}\left(\rho_{t}^{-q-\nu+\mu} \exp \left(\int_{0}^{t}\left(V^{\infty}-V^{\lambda}\right)\left(\rho_{s}\right) d s\right) 1_{\left(t \leq \tau_{r_{\lambda}}\right)}\right),
\end{aligned}
$$

where we slightly abuse the notation and let $\tau_{r}=\tau_{r}^{\rho}=\inf \left\{t \geq 0: \rho_{t} \leq r\right\}$ for any $r>0$. Use the above to see that (D.13) becomes

$$
\begin{aligned}
& I(\lambda)=\left|x-x_{1}\right|^{\nu-\mu} \int_{0}^{\infty} E_{\left|x-x_{1}\right|}^{(2+2 \nu)}\left(\rho_{t}^{-q-\nu+\mu} \exp \left(\int_{0}^{t}\left(V^{\infty}-V^{\lambda}\right)\left(\rho_{s}\right) d s\right) 1_{\left(t \leq \tau_{r_{\lambda}}\right)}\right) d t \\
& =\left|x-x_{1}\right|^{\nu-\mu} E_{\left|x-x_{1}\right|}^{(2+2 \nu)}\left(\int_{0}^{\tau_{r_{\lambda}}} \rho_{t}^{-q-\nu+\mu} \exp \left(\int_{0}^{t}\left(V^{\infty}-V^{\lambda}\right)\left(\rho_{s}\right) d s\right) d t\right),
\end{aligned}
$$


where the second equality is by Fubini's theorem. Now use the scaling of Bessel process and $V^{\infty}, V^{\lambda}$ (recall $r_{\lambda}=\lambda_{0} \lambda^{-\frac{1}{4-d}}$ ) to see that

$$
\begin{aligned}
I(\lambda) & =\left|x-x_{1}\right|^{\nu-\mu} E_{\left|x-x_{1}\right| / r_{\lambda}}^{(2+2 \nu)}\left(\int_{0}^{\tau_{1}} r_{\lambda}^{2-q-\nu+\mu} \rho_{t}^{-q-\nu+\mu} \exp \left(\int_{0}^{t}\left(V^{\infty}-V^{\lambda_{0}^{4-d}}\right)\left(\rho_{s}\right) d s\right) d t\right) \\
& \leq\left|x-x_{1}\right|^{\nu-\mu} E_{\left|x-x_{1}\right| / r_{\lambda}}^{(2+2 \nu)}\left(\int_{0}^{\tau_{1}} r_{\lambda}^{2-q-\nu+\mu} \rho_{t}^{-q-\nu+\mu} \exp \left(\int_{0}^{t} c_{3.1} \lambda_{0}^{-(p-2)} \rho_{s}^{-p} d s\right) d t\right),
\end{aligned}
$$

where the last inequality is by Lemma 3.1.

We interrupt the proof for another auxiliary result from [19].

Lemma D.1. There is some universal constant $c_{D .1}>0$ such that for any $r>0$ with $r<|x|$ and $0<\delta<(p-2)(2-\mu)$ and $2+\mu-\nu<q<6-p$, we have

$$
E_{|x| / r}^{(2+2 \nu)}\left(\int_{0}^{\tau_{1}} \rho_{t}^{-q-\nu+\mu} \exp \left(\int_{0}^{t} \delta \rho_{s}^{-p} d s\right) d t\right) \leq c_{D .1} r^{-2+q+\nu-\mu}|x|^{2-q-\nu+\mu} .
$$

Proof. This is included in the proof of Proposition 6.1 of [19] with $r=r_{\lambda}$. In particular, the above expectation appears in (9.23) of [19] and is bounded by $e J_{i}$ in (9.27) of that paper. Following the inequalities in that work, noting we only need to use Lemma 9.6(b) with $a=1, \gamma>1$ and $\gamma+p-2<1+\nu$ where $2 \gamma=q+\nu-\mu$, we arrive at the above bound.

Returning to (D.15), we choose $\lambda_{0}>0$ so that $c_{3.1} \lambda_{0}^{-(p-2)}<(p-2)(2-\mu)$. If $\lambda$ is sufficiently large so that $r_{\lambda}<\left|x-x_{1}\right|$ we may apply Lemma D.1 to conclude

$$
I(\lambda) \leq\left|x-x_{1}\right|^{\nu-\mu} r_{\lambda}^{2-q-\nu+\mu} c_{D .1}\left|x-x_{1}\right|^{2-q-\nu+\mu} r_{\lambda}^{-2+q+\nu-\mu}=c_{D .1}\left|x-x_{1}\right|^{2-q}<\infty
$$

and we finish the proof of (D.11).

Next we show that for any fixed $T>0$,

$$
\begin{gathered}
\lim _{\lambda \rightarrow \infty} \iint_{0}^{T}\left|B_{t}-x_{1}\right|^{-p} \exp \left(-\int_{0}^{t} V^{\lambda}\left(B_{s}-x_{1}\right) d s\right) 1\left(t \leq T_{r_{\lambda}}^{1}\right) d t d P_{x} \\
=\iint_{0}^{T}\left|B_{t}-x_{1}\right|^{-p} \exp \left(-\int_{0}^{t} V^{\infty}\left(B_{s}-x_{1}\right) d s\right) d t d P_{x}
\end{gathered}
$$

Since we are working under a finite measure $1(t \leq T) d t d P_{x}$, it suffices to show that $\left\{\left|B_{t}-x_{1}\right|^{-p} \exp \left(-\int_{0}^{t} V^{\lambda}\left(B_{s}-x_{1}\right) d s\right) 1\left(t \leq T_{r_{\lambda}}^{1}\right)\right\}$ is a uniformly integrable family indexed by $\lambda$ sufficiently large. This in turn will follow from a $(1+\gamma)$ moment bound for $\gamma>0$ which is uniform in $\lambda$ sufficiently large. Since $p \in(2,3)$, we can pick $\gamma>0$ small such that $q:=p(1+\gamma)<6-p$. Therefore by (D.11) we have

$$
\begin{aligned}
& \iint_{0}^{T}\left|B_{t}-x_{1}\right|^{-p(1+\gamma)} \exp \left(-(1+\gamma) \int_{0}^{t} V^{\lambda}\left(B_{s}-x_{1}\right) d s\right) 1\left(t \leq T_{r_{\lambda}}^{1}\right) d t d P_{x} \\
& \leq \iint_{0}^{\infty}\left|B_{t}-x_{1}\right|^{-q} \exp \left(-\int_{0}^{t} V^{\lambda}\left(B_{s}-x_{1}\right) d s\right) 1\left(t \leq T_{r_{\lambda}}^{1}\right) d t d P_{x}<\infty,
\end{aligned}
$$

and (D.16) follows as noted above.

Use (D.12) with $q=p$ to get

$$
\lim _{T \rightarrow \infty} \iint_{T}^{\infty}\left|B_{t}-x_{1}\right|^{-p} \exp \left(-\int_{0}^{t} V^{\infty}\left(B_{s}-x_{1}\right) d s\right) d t d P_{x}=0 .
$$


We claim that

$$
\lim _{T \rightarrow \infty} \sup _{\lambda>0} \iint_{T}^{\infty}\left|B_{t}-x_{1}\right|^{-p} \exp \left(-\int_{0}^{t} V^{\lambda}\left(B_{s}-x_{1}\right) d s\right) 1_{\left(t \leq T_{r_{\lambda}}^{1}\right)} d t d P_{x}=0 .
$$

Then the proof of (D.9) will follow immediately from (D.16), (D.17) and (D.18).

It remains to prove (D.18). Similar to the derivation of (D.13) and (D.14) with $q=p$, we have

$$
\begin{aligned}
& \iint_{T}^{\infty}\left|B_{t}-x_{1}\right|^{-p} \exp \left(-\int_{0}^{t} V^{\lambda}\left(B_{s}-x_{1}\right) d s\right) 1_{\left(t \leq T_{r_{\lambda}}^{1}\right)} d t d P_{x} \\
& =\left|x-x_{1}\right|^{\nu-\mu} \int_{T}^{\infty} E_{\left|x-x_{1}\right|}^{(2+2 \nu)}\left(\rho_{t}^{-p-\nu+\mu} \exp \left(\int_{0}^{t}\left(V^{\infty}-V^{\lambda}\right)\left(\rho_{s}\right) d s\right) 1_{\left(t \leq \tau_{r_{\lambda}}\right)}\right) d t .
\end{aligned}
$$

Use $p=\mu+\nu$ to see that the integrand of the right-hand side term of the above equals

$$
\begin{aligned}
& E_{\left|x-x_{1}\right|}^{(2+2 \nu)}\left(\rho_{t}^{-2 \nu} \exp \left(\int_{0}^{t}\left(V^{\infty}-V^{\lambda}\right)\left(\rho_{s}\right) d s\right) 1\left(t \leq \tau_{r_{\lambda}}\right)\right) \\
& \leq\left(E_{\left|x-x_{1}\right|}^{(2+2 \nu)}\left(\rho_{t}^{-2 \nu}\right)\right)^{1 / 2} \times\left(E_{\left|x-x_{1}\right|}^{(2+2 \nu)}\left(\rho_{t}^{-2 \nu} \exp \left(\int_{0}^{t} 2\left(V^{\infty}-V^{\lambda}\right)\left(\rho_{s}\right) d s\right) 1\left(t \leq \tau_{r_{\lambda}}\right)\right)\right)^{1 / 2},
\end{aligned}
$$

where in the inequality we have applied Cauchy-Schwarz inequality.

For the first term on the right-hand side of (D.20), we use the scaling of Bessel process to get

$$
E_{\left|x-x_{1}\right|}^{(2+2 \nu)}\left(\rho_{t}^{-2 \nu}\right)=t^{-\nu} E_{\left|x-x_{1}\right|}^{(2+2 \nu)}\left(\rho_{1}^{-2 \nu}\right):=t^{-\nu} C\left(\nu,\left|x-x_{1}\right|\right)
$$

where the finiteness of $E_{\left|x-x_{1}\right|}^{(2+2 \nu)}\left(\rho_{1}^{-2 \nu}\right)$ follows easily from the known transition density of Bessel process (see, e.g. Chp. XI of [21]). For the second term on the right-hand side of (D.20), by (2.C) of [24] one can conclude that for any $r>\varepsilon>0$,

$$
\left.P_{r}^{(2-2 \nu)}\right|_{\mathcal{F}_{\tau_{\varepsilon} \wedge t}^{\rho}} ^{\rho}=\left.\frac{r^{2 \nu}}{\rho_{\tau_{\varepsilon} \wedge t}^{2 \nu}} P_{r}^{(2+2 \nu)}\right|_{\mathcal{F}_{\tau_{\varepsilon} \wedge t}^{\rho}} .
$$

Use the above to get

$$
\begin{aligned}
& E_{\left|x-x_{1}\right|}^{(2+2 \nu)}\left(\rho_{t}^{-2 \nu} \exp \left(\int_{0}^{t} 2\left(V^{\infty}-V^{\lambda}\right)\left(\rho_{s}\right) d s\right) 1\left(t \leq \tau_{r_{\lambda}}\right)\right) \\
& =E_{\left|x-x_{1}\right|}^{(2+2 \nu)}\left(\rho_{t \wedge \tau_{r_{\lambda}}}^{-2 \nu} \exp \left(\int_{0}^{t \wedge \tau_{r_{\lambda}}} 2\left(V^{\infty}-V^{\lambda}\right)\left(\rho_{s}\right) d s\right) 1\left(t \leq t \wedge \tau_{r_{\lambda}}\right)\right) \\
& =\left|x-x_{1}\right|^{-2 \nu} E_{\left|x-x_{1}\right|}^{(2-2 \nu)}\left(\exp \left(\int_{0}^{t \wedge \tau_{r_{\lambda}}} 2\left(V^{\infty}-V^{\lambda}\right)\left(\rho_{s}\right) d s\right) 1\left(t \leq t \wedge \tau_{r_{\lambda}}\right)\right) \\
& \leq\left|x-x_{1}\right|^{-2 \nu} E_{\left|x-x_{1}\right|}^{(2-2 \nu)}\left(\exp \left(\int_{0}^{\tau_{r_{\lambda}}} 2\left(V^{\infty}-V^{\lambda}\right)\left(\rho_{s}\right) d s\right)\right) \\
& =\left|x-x_{1}\right|^{-2 \nu} E_{\left|x-x_{1}\right|}^{(2+2 \nu)}\left(\exp \left(\int_{0}^{\tau_{r_{\lambda}}} 2\left(V^{\infty}-V^{\lambda}\right)\left(\rho_{s}\right) d s\right) \mid \tau_{r_{\lambda}}<\infty\right) \\
& \leq\left|x-x_{1}\right|^{-2 \nu} C_{3.5}\left(\lambda_{0}, \nu, 2\right),
\end{aligned}
$$

where the last equality is by Corollary 7.3 and in the last inequality we have used Lemma 3.5 with $\lambda_{0}>c_{3.5}$ and $\gamma=2$. Now we conclude from (D.20), (D.21) and (D.23) that

$$
\begin{aligned}
& E_{\left|x-x_{1}\right|}^{(2+2 \nu)}\left(\rho_{t}^{-2 \nu} \exp \left(\int_{0}^{t}\left(V^{\infty}-V^{\lambda}\right)\left(\rho_{s}\right) d s\right) 1\left(t \leq \tau_{r_{\lambda}}\right)\right) \\
& \leq C\left(\nu,\left|x-x_{1}\right|\right)^{1 / 2} t^{-\nu / 2} C_{3.5}\left(\lambda_{0}, \nu, 2\right)^{1 / 2}\left|x-x_{1}\right|^{-\nu}=C\left(\left|x-x_{1}\right|\right) t^{-\nu / 2} .
\end{aligned}
$$


Returning to (D.19), we apply the above to get

$$
\begin{aligned}
& \iint_{T}^{\infty}\left|B_{t}-x_{1}\right|^{-p} \exp \left(-\int_{0}^{t} V^{\lambda_{1}}\left(B_{s}-x_{1}\right) d s\right) 1\left(t \leq T_{r_{\lambda_{1}}}^{1}\right) d t d P_{x} \\
& \leq\left|x-x_{1}\right|^{\nu-\mu} \int_{T}^{\infty} C\left(\left|x-x_{1}\right|\right) t^{-\nu / 2} d t \\
& \leq C\left(\left|x-x_{1}\right|\right) \cdot(\nu / 2-1)^{-1} T^{1-\nu / 2} \rightarrow 0 \text { as } T \rightarrow \infty,
\end{aligned}
$$

thus giving (D.18). The proof is then complete.

Proof of Proposition 4.3(ii). For any $x_{1} \neq x_{2}$, we fix $x \neq x_{1}, x_{2}$. In order to find the limit of $\lambda_{1}^{1+\alpha} \varepsilon^{-(p-2)}\left(-W_{1,2}^{\vec{\lambda}, \vec{x}, \varepsilon}(x)\right)$ as $\lambda_{1} \rightarrow \infty, \varepsilon \downarrow 0$, by Lemma 9.4, it suffices to find the limits of the following as $\lambda_{1} \rightarrow \infty, \varepsilon \downarrow 0$.

$$
\begin{aligned}
K_{1}+ & K_{2} \equiv \frac{\lambda_{1}^{1+\alpha}}{\varepsilon^{p-2}} E_{x}\left(\int_{0}^{T_{\lambda_{1}, \varepsilon}} \prod_{i=1}^{2} W_{i}^{\vec{\lambda}, \vec{x}, \varepsilon}\left(B_{t}\right) \exp \left(-\int_{0}^{t} W^{\vec{\lambda}, \vec{x}, \varepsilon}\left(B_{s}\right) d s\right) d t\right) \\
+ & \frac{\lambda_{1}^{1+\alpha}}{\varepsilon^{p-2}} E_{x}\left(\exp \left(-\int_{0}^{T_{\lambda_{1}, \varepsilon}} W^{\vec{\lambda}, \vec{x}, \varepsilon}\left(B_{s}\right) d s\right) 1_{\left(T_{\lambda_{1}, \varepsilon}<\infty\right)}\left(-W_{1,2}^{\vec{\lambda}, \vec{x}, \varepsilon}\left(B\left(T_{\lambda_{1}, \varepsilon}\right)\right)\right)\right) .
\end{aligned}
$$

In the above $T_{\lambda_{1}, \varepsilon}=T_{r_{\lambda_{1}}}^{1} \wedge T_{2 \varepsilon}^{2}$ where $T_{r_{\lambda_{1}}}^{1}=\inf \left\{t \geq 0:\left|B_{t}-x_{1}\right| \leq r_{\lambda_{1}}\right\}$ and $T_{2 \varepsilon}^{2}=\inf \{t \geq$ $\left.0:\left|B_{t}-x_{2}\right| \leq 2 \varepsilon\right\}$. Here $r_{\lambda_{1}}=\lambda_{0} \lambda_{1}^{-\frac{1}{4-d}}$ and we will choose $\lambda_{0}$ to be some fixed large constant below. Let $\varepsilon>0$ small and $\lambda_{1}>0$ large so that

$$
0<4\left(r_{\lambda_{1}} \vee \varepsilon\right)<\min \left\{\left|x_{1}-x\right|,\left|x_{2}-x\right|,\left|x_{1}-x_{2}\right|\right\}
$$

We first consider $K_{2}$. On $\left\{T_{\lambda_{1}, \varepsilon}<\infty\right\}$, by considering $T_{\lambda_{1}, \varepsilon}=T_{r_{\lambda_{1}}}^{1}<T_{2 \varepsilon}^{2}$ we may set $x_{\lambda}(\omega)=B\left(T_{\lambda_{1}, \varepsilon}\right)=B\left(T_{r_{\lambda_{1}}}^{1}\right)$ so that $\left|x_{\lambda}-x_{1}\right|=r_{\lambda_{1}}$ and hence $\left|x_{2}-x_{\lambda}\right| \geq \Delta / 2$ where $\Delta=\left|x_{1}-x_{2}\right|$. Lemma 9.1 and the above imply

$$
\left(-W_{1,2}^{\vec{\lambda}, \vec{x}, \varepsilon}\left(B\left(T_{\lambda_{1}, \varepsilon}\right)\right)\right) \leq 2 \lambda_{1}^{-1}\left|B\left(T_{\lambda_{1}, \varepsilon}\right)-x_{2}\right|^{-p} \varepsilon^{p-2} \leq 2^{p+1} \Delta^{-p} \lambda_{1}^{-1} \varepsilon^{p-2} .
$$

Similarly by considering $T_{\lambda_{1}, \varepsilon}=T_{2 \varepsilon}^{2}<T_{r_{\lambda_{1}}}^{1}$ we have $\left|B\left(T_{2 \varepsilon}^{2}\right)-x_{1}\right| \geq \Delta / 2$ and hence by Lemma 9.1,

$$
\left(-W_{1,2}^{\vec{\lambda}, \vec{x}, \varepsilon}\left(B\left(T_{\lambda_{1}, \varepsilon}\right)\right)\right) \leq 2 \lambda_{2}^{-1} c_{3.2} \lambda_{1}^{-(1+\alpha)}\left|B\left(T_{\lambda_{1}, \varepsilon}\right)-x_{1}\right|^{-p} \leq 2^{p+1} \Delta^{-p} c_{3.2} \lambda_{2}^{-1} \lambda_{1}^{-(1+\alpha)} .
$$

This shows that

$$
\begin{aligned}
K_{2} & \leq \frac{\lambda_{1}^{1+\alpha}}{\varepsilon^{p-2}} 2^{p+1} \Delta^{-p} \lambda_{1}^{-1} \varepsilon^{p-2} E_{x}\left(1_{\left(T_{r_{\lambda_{1}}}^{1}<\infty\right)} 1_{\left(T_{r_{\lambda_{1}}}^{1}<T_{2 \varepsilon}^{2}\right)} \exp \left(-\int_{0}^{T_{r_{\lambda_{1}}}^{1}} W^{\vec{\lambda}, \vec{x}, \varepsilon}\left(B_{s}\right) d s\right)\right) \\
& +\frac{\lambda_{1}^{1+\alpha}}{\varepsilon^{p-2}} 2^{p+1} \Delta^{-p} c_{3.2} \lambda_{2}^{-1} \lambda_{1}^{-(1+\alpha)} E_{x}\left(1_{\left(T_{2 \varepsilon}^{2}<\infty\right)} 1_{\left(T_{2 \varepsilon}^{2}<T_{r_{\lambda_{1}}}^{1}\right)} \exp \left(-\int_{0}^{T_{2 \varepsilon}^{2}} W^{\vec{\lambda}, \vec{x}, \varepsilon}\left(B_{s}\right) d s\right)\right) .
\end{aligned}
$$

By (8.8), for all $x$ so that $x \neq x_{1}$ and $\left|x-x_{2}\right|>\varepsilon$ we have

$$
W^{\vec{\lambda}, \vec{x}, \varepsilon}(x) \geq V^{\lambda_{1}}\left(x-x_{1}\right) \vee V^{\infty}\left(x-x_{2}\right)
$$

Let $\tau_{r}=\inf \left\{t:\left|B_{t}\right| \leq r\right\}$. Use the above to see that 


$$
\begin{aligned}
& E_{x}\left(1_{\left(T_{r_{\lambda_{1}}}^{1}<\infty\right)} 1_{\left(T_{r_{\lambda_{1}}}^{1}<T_{2 \varepsilon}^{2}\right)} \exp \left(-\int_{0}^{T_{r_{\lambda_{1}}}^{1}} W^{\vec{\lambda}, \vec{x}, \varepsilon}\left(B_{s}\right) d s\right)\right) \\
\leq & E_{x-x_{1}}\left(1\left(\tau_{r_{\lambda_{1}}}<\infty\right) \exp \left(-\int_{0}^{\tau_{r_{\lambda_{1}}}} V^{\lambda_{1}}\left(B_{s}\right) d s\right)\right) \leq r_{\lambda_{1}}^{p}\left|x-x_{1}\right|^{-p} C_{3.5}\left(\lambda_{0}, \nu, 1\right),
\end{aligned}
$$

where the last line follows in a similar way to the derivation of (C.3) by choosing $\lambda_{0}>c_{3.5}$.

Similarly by (D.28) and (C.29) we have

$$
\begin{aligned}
& \left.E_{x}\left(1_{\left(T_{2 \varepsilon}^{2}<\infty\right)} 1_{\left(T_{2 \varepsilon}^{2}<T_{r_{1}}^{1}\right.}\right) \exp \left(-\int_{0}^{T_{2 \varepsilon}^{2}} W^{\vec{\lambda}, \vec{x}, \varepsilon}\left(B_{s}\right) d s\right)\right) \\
\leq & E_{x-x_{2}}\left(1\left(\tau_{2 \varepsilon}<\infty\right) \exp \left(-\int_{0}^{\tau_{2 \varepsilon}} V^{\infty}\left(B_{s}\right) d s\right)\right)=\left(2 \varepsilon /\left|x-x_{1}\right|\right)^{p} .
\end{aligned}
$$

Apply (D.29) and (D.30) in (D.27) to get

$$
\begin{aligned}
K_{2} \leq & \frac{\lambda_{1}^{1+\alpha}}{\varepsilon^{p-2}} 2^{p+1} \Delta^{-p} \lambda_{1}^{-1} \varepsilon^{p-2} r_{\lambda_{1}}^{p}\left|x-x_{1}\right|^{-p} C_{3.5}\left(\lambda_{0}, \nu, 1\right) \\
& +\frac{\lambda_{1}^{1+\alpha}}{\varepsilon^{p-2}} 2^{p+1} \Delta^{-p} c_{3.2} \lambda_{2}^{-1} \lambda_{1}^{-(1+\alpha)}\left(2 \varepsilon /\left|x-x_{1}\right|\right)^{p} \\
\leq & C \Delta^{-p} \lambda_{0}^{p}\left|x-x_{1}\right|^{-p} \lambda_{1}^{-\frac{2}{4-d}}+C \lambda_{2}^{-1} \Delta^{-p}\left|x-x_{2}\right|^{-p} \varepsilon^{2} \rightarrow 0
\end{aligned}
$$

as $\lambda_{1} \rightarrow \infty, \varepsilon \downarrow 0$, where in the last equality we have used the definitions of $r_{\lambda_{1}}$ and $\alpha$.

Now we will turn to $K_{1}$. Recall

$$
K_{1}=\iint_{0}^{\infty} \frac{\lambda_{1}^{1+\alpha}}{\varepsilon^{p-2}} W_{1}^{\vec{\lambda}, \vec{x}, \varepsilon}\left(B_{t}\right) W_{2}^{\vec{\lambda}, \vec{x}, \varepsilon}\left(B_{t}\right) \exp \left(-\int_{0}^{t} W^{\vec{\lambda}, \vec{x}, \varepsilon}\left(B_{s}\right) d s\right) 1\left(t \leq T_{\lambda_{1}, \varepsilon}\right) d t d P_{x} .
$$

By Proposition 4.3 and Lemma 8.1, for $L e b \times P_{x}$-a.e. $(t, \omega)$, we have

$$
\begin{gathered}
\lim _{\lambda_{1} \rightarrow \infty, \varepsilon \downarrow 0} \frac{\lambda_{1}^{1+\alpha}}{\varepsilon^{p-2}} W_{1}^{\vec{\lambda}, \vec{x}, \varepsilon}\left(B_{t}\right) W_{2}^{\vec{\lambda}, \vec{x}, \varepsilon}\left(B_{t}\right) \exp \left(-\int_{0}^{t} W^{\vec{\lambda}, \vec{x}, \varepsilon}\left(B_{s}\right) d s\right) 1\left(t \leq T_{\lambda_{1}, \varepsilon}\right) \\
=K_{4.1} C_{4.1}\left(\lambda_{2}\right) U_{1}^{\infty, \vec{x}}\left(B_{t}\right) U_{2}^{\text {के, } \vec{x}}\left(B_{t}\right) \exp \left(-\int_{0}^{t} V^{\vec{\infty}, \vec{x}}\left(B_{s}\right) d s\right) .
\end{gathered}
$$

Use the bounds (5.4), (5.5) and (D.28) to see that

$$
\begin{gathered}
\quad \frac{\lambda_{1}^{1+\alpha}}{\varepsilon^{p-2}} W_{1}^{\vec{\lambda}, \vec{x}, \varepsilon}\left(B_{t}\right) W_{2}^{\vec{\lambda}, \vec{x}, \varepsilon}\left(B_{t}\right) \exp \left(-\int_{0}^{t} W^{\vec{\lambda}, \vec{x}, \varepsilon}\left(B_{s}\right) d s\right) 1\left(t \leq T_{\lambda_{1}, \varepsilon}\right) \\
\leq c_{3.2}\left|B_{t}-x_{1}\right|^{-p}\left|B_{t}-x_{2}\right|^{-p} \exp \left(-\int_{0}^{t} W^{\vec{\lambda}, \vec{x}, \varepsilon}\left(B_{s}\right) d s\right) 1\left(t \leq T_{\lambda_{1}, \varepsilon}\right) \\
=c_{3.2} \sum_{i=1}^{2}\left|B_{t}-x_{1}\right|^{-p}\left|B_{t}-x_{2}\right|^{-p} 1\left(\left|B_{t}-x_{i}\right| \leq\left|B_{t}-x_{3-i}\right|\right) \\
\exp \left(-\int_{0}^{t} W^{\vec{\lambda}, \vec{x}, \varepsilon}\left(B_{s}\right) d s\right) 1\left(t \leq T_{\lambda_{1}, \varepsilon}\right) \\
\leq c_{3.2} 2^{p} \Delta^{-p}\left|B_{t}-x_{1}\right|^{-p} \exp \left(-\int_{0}^{t} V^{\lambda_{1}}\left(B_{s}-x_{1}\right) d s\right) 1\left(t \leq T_{r_{\lambda_{1}}}^{1}\right) \\
+c_{3.2} 2^{p} \Delta^{-p}\left|B_{t}-x_{2}\right|^{-p} \exp \left(-\int_{0}^{t} V^{\infty}\left(B_{s}-x_{2}\right) d s\right)
\end{gathered}
$$

where we have used $\left|B_{t}-x_{3-i}\right|>\Delta / 2$ on $\left\{\left|B_{t}-x_{i}\right| \leq\left|B_{t}-x_{3-i}\right|\right\}$ and $T_{\lambda_{1}, \varepsilon} \leq T_{r_{\lambda_{1}}}^{1}$ in 
the last inequality. It is clear that for $L e b \times P_{x}$-a.e. $(t, \omega)$,

$$
\begin{aligned}
& \lim _{\lambda_{1} \rightarrow \infty}\left|B_{t}-x_{1}\right|^{-p} \exp \left(-\int_{0}^{t} V^{\lambda_{1}}\left(B_{s}-x_{1}\right) d s\right) 1\left(t \leq T_{r_{\lambda_{1}}}^{1}\right) \\
& =\left|B_{t}-x_{1}\right|^{-p} \exp \left(-\int_{0}^{t} V^{\infty}\left(B_{s}-x_{1}\right) d s\right) .
\end{aligned}
$$

By (D.9) we have

$$
\begin{gathered}
\lim _{\lambda_{1} \rightarrow \infty} \iint_{0}^{\infty}\left|B_{t}-x_{1}\right|^{-p} \exp \left(-\int_{0}^{t} V^{\lambda_{1}}\left(B_{s}-x_{1}\right) d s\right) 1\left(t \leq T_{r_{\lambda_{1}}}^{1}\right) d t d P_{x} \\
=\iint_{0}^{\infty}\left|B_{t}-x_{1}\right|^{-p} \exp \left(-\int_{0}^{t} V^{\infty}\left(B_{s}-x_{1}\right) d s\right) d t d P_{x}<\infty,
\end{gathered}
$$

and by (D.12) with $q=p$ we have

$$
\iint_{0}^{\infty}\left|B_{t}-x_{2}\right|^{-p} \exp \left(-\int_{0}^{t} V^{\infty}\left(B_{s}-x_{2}\right) d s\right) d t d P_{x}<\infty .
$$

In view of (D.32), (D.33), (D.34), (D.35) and (D.36), a generalized the dominated convergence theorem Theorem (see, e.g., Exercise 20 of Chp. 2 of [4]) implies that

$$
\begin{aligned}
& \lim _{\lambda_{1} \rightarrow \infty, \varepsilon \downarrow 0} K_{1}=\lim _{\lambda_{1} \rightarrow \infty, \varepsilon \downarrow 0} \iint_{0}^{\infty} \frac{\lambda_{1}^{1+\alpha}}{\varepsilon^{p-2}} W_{1}^{\vec{\lambda}, \vec{x}, \varepsilon}\left(B_{t}\right) W_{2}^{\vec{\lambda}, \vec{x}, \varepsilon}\left(B_{t}\right) \\
& \exp \left(-\int_{0}^{t} W^{\vec{\lambda}, \vec{x}, \varepsilon}\left(B_{s}\right) d s\right) 1_{\left\{t \leq T_{\lambda_{1}, \varepsilon}\right\}} d t d P_{x} \\
= & K_{4.1} C_{4.1}\left(\lambda_{2}\right) \iint_{0}^{\infty} U_{1}^{\vec{\infty}, \vec{x}}\left(B_{t}\right) U_{2}^{\vec{\infty}, \vec{x}}\left(B_{t}\right) \exp \left(-\int_{0}^{t} V^{\vec{\infty}, \vec{x}}\left(B_{s}\right) d s\right) d t d P_{x} \\
= & K_{4.1} C_{4.1}\left(\lambda_{2}\right)\left(-U_{1,2}^{\vec{\infty}, \vec{x}}(x)\right),
\end{aligned}
$$

where the last is by (1.17). The proof will then be finished by Lemma 9.4, (D.25), (D.31) and the above.

\section{References}

[1] R. Ash. Probability and Measure Theory. With Contributions by Catherine Doléans-Dade. Second edition. Harcourt/Academic Press, Burlington, MA (2000). MR-1810041

[2] D. Dawson, I. Iscoe and E. Perkins. Super-Brownian motion: Path properties and hitting probabilities. Prob. Th. Rel. Fields, 83, 135-205 (1989). MR-1012498

[3] W. Feller. An Introduction to Probability Theory and Its Applications. Vol. II. Second edition. John Wiley \& Sons, Inc., New York-London-Sydney (1971). MR-0270403

[4] G. Folland. Real Analysis: Modern Techniques and Their Applications. Second edition. Pure and Applied Mathematics (New York). A Wiley-Interscience Publication. John Wiley \& Sons, Inc., New York (1999). MR-1681462

[5] A. Göing-Jaeschke and M. Yor. A survey and some generalizations of Bessel processes. Bernoulli, 9(2), 313-349 (2003). MR-1997032

[6] J. Hong. Renormalization of local times of super-Brownian motion. Electron. J. Probab., 23(109), 1-45 (2018). MR-3878134

[7] J. Hong. Improved Hölder continuity near the boundary of one-dimensional super-Brownian motion. Electron. Commun. Probab., 24(28), 12 (2019). MR-3962478

[8] J. Hong. Exit measure, local time and a boundary local time of super-Brownian motion. arXiv:2001.07269 (2020). Submitted. MR-3878134

[9] J. Hong, L. Mytnik and E. Perkins. On the topological boundary of the range of super-Brownian motion. Ann. Probab., 48(3), 1168-1201 (2020). MR-4112711 
[10] J. Hong, L. Mytnik and E. Perkins. Supplement to "On the topological boundary of the range of super-Brownian motion.” Ann. Probab., 48(3), 1168-1201 (2020). MR-4112711

[11] T. Hughes. A boundary local time for one-dimensional super-Brownian motion and applications. Electron. J. Probab., 24(54), 1-58 (2019). MR-3968716

[12] N. Ikeda and S. Watanabe. Stochastic Differential Equations and Diffusion Processes. Second edition. North-Holland Mathematical Library, vol. 24. North-Holland Publishing Co., Amsterdam; Kodansha, Ltd., Tokyo (1989). MR-1011252

[13] I. Iscoe. On the supports of measure-valued critical branching Brownian motion. Ann. Probab., 16(1), 200-221 (1988). MR-0920265

[14] G. Lawler. Notes on the Bessel process. Draft (2018).

[15] J.F. Le Gall. The Brownian snake and solutions of $\Delta u=u^{2}$ in a domain. Probab. Theory Relat. Fields, 102, 393-432 (1995). MR-1339740

[16] J.F. Le Gall. Spatial Branching Processes, Random Snakes and Partial Differential Equations. Lectures in Mathematics. ETH, Zurich/Birkhäuser, Basel (1999). MR-1714707

[17] J.F. Le Gall. Bessel processes, the Brownian snake and super-Brownian motion. In: DonatiMartin C., Lejay A., Rouault A. (eds.), In Memoriam Marc Yor - Séminaire de Probabilités XLVII. Lecture Notes in Mathematics, vol. 2137. Springer, Cham (2015). MR-3444295

[18] P. Mörters and Y. Peres. Brownian motion. With an appendix by Oded Schramm and Wendelin Werner. Cambridge Series in Statistical and Probabilistic Mathematics, vol. 30. Cambridge University Press, Cambridge (2010). MR-2604525

[19] L. Mytnik and E. Perkins. The dimension of the boundary of super-Brownian motion. Prob. Th. Rel. Fields, 174, 821-885 (2019). MR-3980306

[20] E.A. Perkins. Dawson-Watanabe superprocesses and measure-valued diffusions. In: Ecole d'Eté de Probabilités de Saint Flour 1999. Lectures on Probability Theory and Statistics, vol. 1781. Springer, Berlin (2002). MR-1915445

[21] D. Revuz and M. Yor. Continuous Martingales and Brownian Motion. Springer, Berlin (1994). MR-1303781

[22] L.C.G. Rogers and D. Williams. Diffusions, Markov Processes and Martingales Vol. 2. Cambridge University Press, Cambridge (1994). MR-1780932

[23] S. Sugitani. Some properties for the measure-valued branching diffusion processes. J. Math. Soc. Japan, 41, 437-462 (1989). MR-0999507

[24] M. Yor. On some exponential functionals of Brownian motion. Adv. Appl. Prob., 24, 509-531 (1992). MR-1174378

Acknowledgments. This work was done as part of the author's graduate studies at the University of British Columbia. I would like to thank my supervisor, Professor Edwin Perkins, for suggesting this problem and for the helpful discussions and suggestions throughout this work. I also thank the anonymous referee for the careful reading of the manuscript. 